This electronic thesis or dissertation has been downloaded from the King's Research Portal at https://kclpure.kcl.ac.uk/portal/

\title{
Multifocal Multiphoton Microscopy with Adaptive Optical Correction
}

\author{
Pereira Coelho, Simao Pedro
}

Awarding institution:

King's College London

The copyright of this thesis rests with the author and no quotation from it or information derived from it may be published without proper acknowledgement.

\section{END USER LICENCE AGREEMENT}

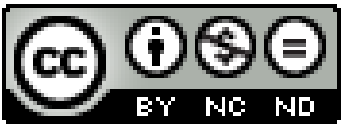

Unless another licence is stated on the immediately following page this work is licensed

under a Creative Commons Attribution-NonCommercial-NoDerivatives 4.0 International

licence. https://creativecommons.org/licenses/by-nc-nd/4.0/

You are free to copy, distribute and transmit the work

Under the following conditions:

- $\quad$ Attribution: You must attribute the work in the manner specified by the author (but not in any way that suggests that they endorse you or your use of the work).

- $\quad$ Non Commercial: You may not use this work for commercial purposes.

- $\quad$ No Derivative Works - You may not alter, transform, or build upon this work.

Any of these conditions can be waived if you receive permission from the author. Your fair dealings and other rights are in no way affected by the above.

Take down policy

If you believe that this document breaches copyright please contact librarypure@kcl.ac.uk providing details, and we will remove access to the work immediately and investigate your claim. 
KING'S COLLEGE LONDON

\section{Multifocal Multiphoton Microscopy with Adaptive Optical Correction}

Thesis submitted to the University of London in partial fulfilment of the degree of Doctor of Philosophy

\section{Simao Pedro Pereira Coelho}

Supervisors:

Dr Simon M. Ameer-Beg

Dr Frederic Festy

Division of Cancer Studies \& Randall Division of Cell and Molecular Biophysics,

Richard Dimbleby Research Laboratory,

New Hunt's House,

Guy's Campus

King's College London 
The only reason for time is so that

everything doesn't happen at once.

Albert Einstein 


\section{ABSTRACT}

Multiphoton microscopy (MPM) is a remarkably versatile technique in biological imaging. MPM provides increased depth over confocal imaging and can be combined with other imaging techniques such as fluorescence lifetime imaging microscopy (FLIM), adding functional information. FLIM read-out is relatively straightforward using time-correlated single photon counting (TCSPC). Fluorescence lifetime detection enhances the power of multiphoton imaging to allow three dimensional, concentration independent, measurements of environmental parameters such as $\mathrm{pH}$, Oxygen tension and $\mathrm{Ca}^{2+}$ in addtion to the interaction or conformational modification of proteins by Förster resonant energy transfer (FRET); the latter is a particular focus of the Dimbleby research groups at King's College London. However, there are significant limitations in both FLIM and MM. Limitations of TCSPC-FLIM include prolonged acquisition times along with signal and resolution degradation as a function of depth. This thesis demonstrates advancements multiphoton fluorescence lifetime imaging through improvements in two principal areas: speed and resolution at depth.

In order to improve acquistion rates a multifocal multiphoton microscope (MMM) capable of rapid, parallelized TCSPC-FLIM was developed - MegaFLI. Acquisitions demonstrate rapid 3-dimensional, high temporal resolution FLIM in vivo Zebrafish. Performed by massively parallel excitation/detection the speed is signficantly improved by a factor of 64 .

In parallel to the MegaFLI project, a second microscope employing adaptive optical correction has been developed. The introduction of Adaptive Optics (AO) serves to improve imaging quality by counteracting the refractive index heterogeneities introduced by the sample, limiting the imaging depth. Incorporated with a single beam scanning FLIM system, a pupil-segmentation AO-TCSPC-FLIM demonstrates improved signal-to-noise ratio (SNR) and resolution, permiting a more accurate determination of fluorescent lifetime in turbid media. 
DECLARATION OF AUTHORSHIP

The work present is my own work with the exception of TRI2, which was developed by Dr. Paul Barber; the weighted Gerchberg-Saxton algorithm and the alignment and stitching algorithm developed by Dr. Simon Poland; SPAD interface developed by Dr. Nikola Krstajic; Nikon Ti-E control developed by Dr. Daniel Mattews and Dr. Simon Poland; Zebrafish preparation performed by Nicole Nicholas; cell preparation performed by Dr. James Moneypenny; tissue preparation performed by Dr. Gregory Weismann. Contributions are indicated in the text. 


\section{TABLE OF CONTENTS}

Abstract

Declaration of Authorship ...................................................................................

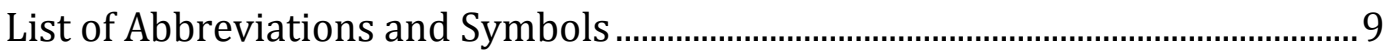

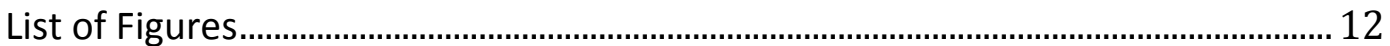

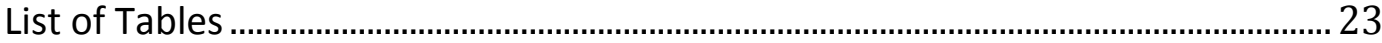

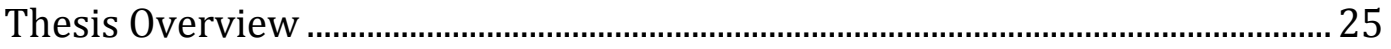

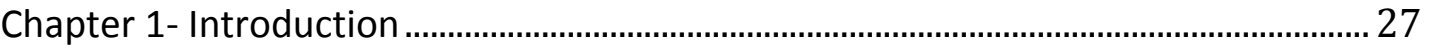

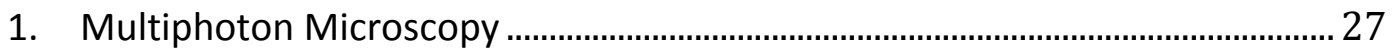

2. Key Components of a Multiphoton System ............................................................ 32

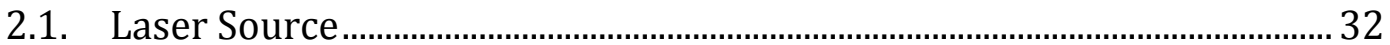

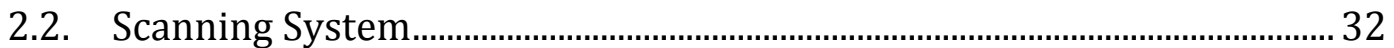

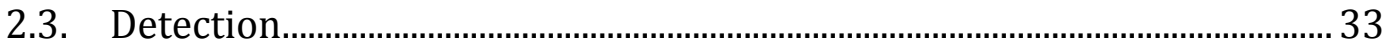

3. Time Correlated Single Photon Counting ............................................................... 33

4. Multi-detector and Multi-wavelength ................................................................ 36

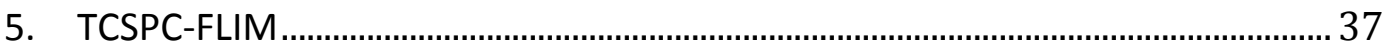

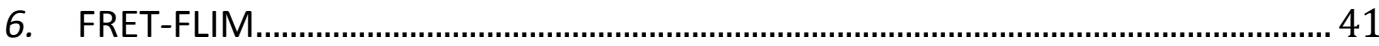

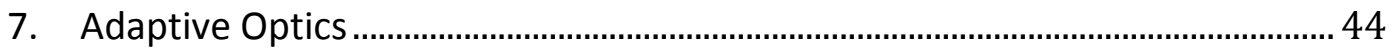

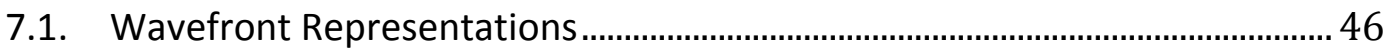

7.2. Analytical Wavefront Representation ……....................................................... 47

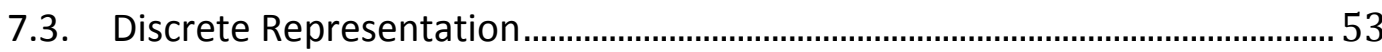

7.4. Wavefront Reconstruction Methods ............................................................... 53 
7.4.1. Wavefront Sensors

7.4.2. Shack-Hartmann Wavefront Sensor .............................................................. 54

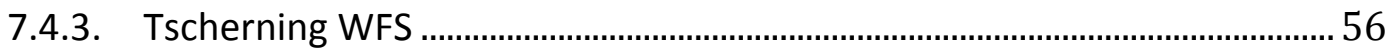

7.5. Wavefront Reconstruction and Analysis....................................................... 57

7.6. Wavefront Calculation: Modal Reconstruction using Zernike Polynomials 58

7.7. Wavefront Calculation: Zonal Reconstruction ..................................................59

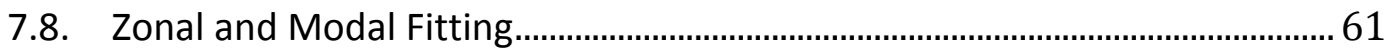

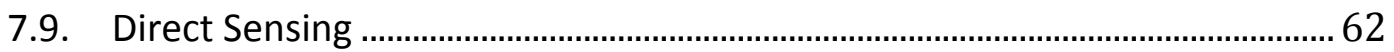

CHAPTER 2 - Multifocal Multiphoton Fluorescence Lifetime Microscopy .................... 63

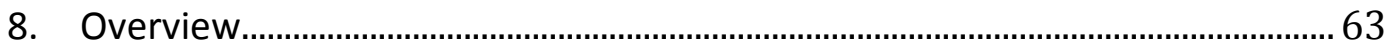

9. Time Resolved Detection System for parallelised TCSPC ...................................66

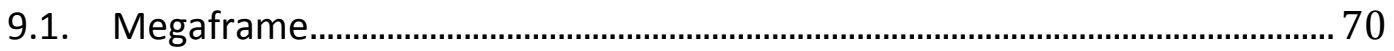

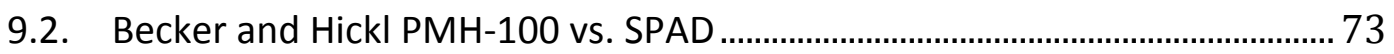

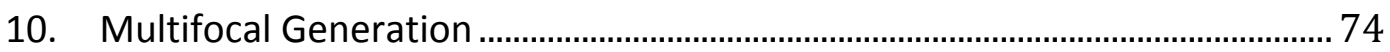

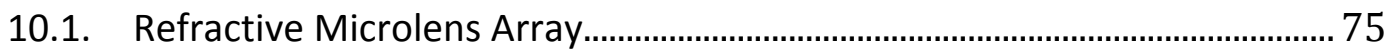

10.2. Programmable Beamlet generation using a Spatial Light Modulator .........82

10.3. Spatial Light Modulator Wavelength Calibration ........................................... 83

10.4. Huygens-Fresnel Diffractive Lens Array........................................................... 87

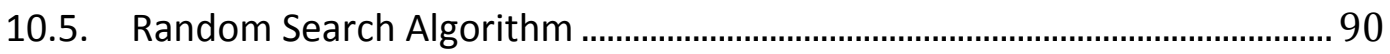

10.6. Holographic Multifocal Generation (Gerchberg-Saxton Algorithm).......... 95

10.7. Comparison Between Multifocal Generation Approaches...........................99

11. Multifocal Multiphoton FLIM System …......................................................... 101 
11.1. Optical System Arrangement

11.2. Optical Design Rationale

11.3. Optical Layout: Descanned Detection 105

11.4. Electronic Control 106

11.5. Megaframe Readout 106

12. Multifocal Multiphoton FLIM Developmental Stages 107

12.1. Multifocal Multiphoton FLIM: Non-Descanned Detection (Developmental Stage I) 108

12.2. Multifocal Multiphoton flim: Descanned Detection (Developmental Stage II)

12.2.1. Beamlet Optimization

12.2.2. Position Optimization

12.2.3. Weighted Gerchberg-Saxton Algorithm

13. Data Analysis

13.1. Image Stitching and Alignment

13.2. TCSPC-FLIM Image Analysis

14. Results: Demonstration of Multifocal Multiphoton FLIM.

14.1. Beam Scanned Multifocal Multphoton FLIM: Descanned Detection ...... 123

14.2. Optical Resolution

14.3. Multifocal FLIM using Fixed Sample

14.4. Weighted Gerchberg-Saxton Illumination

14.5. Selective Beamlet Scanning

14.6. Multifocal FLIM in vitro 
15. Discussion

16. Future Of The Project

17. Overview

17.1. Pupil-Segmentation Approach to Adaptive Optics

18. Experimental Procedure

18.1. Optical Design

18.2. SLM Calibration

18.3. Pupil-Segmentation Description.

19. Results.

19.1. Simulations

19.2. System Correction

20. Sample Aberration Correction.

20.1. FLIM with Adaptive Optics

20.2. Adaptive Optics in vivo

21. Discussion

22. Future of the Project

Chapter 4 - Conclusion .

23. Summary

24. Future Prospects

25. Conclusion 
Appendix B -- Zebrafish for in vivo modelling .............................................................. 178

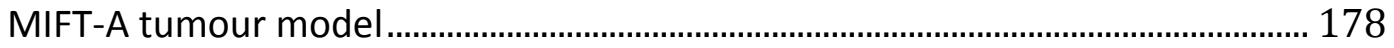

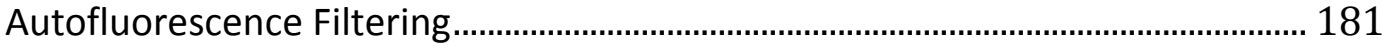

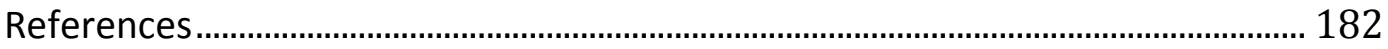




\begin{tabular}{ll} 
AO & Adaptive Optics \\
APD & Avalanche Photo Diode \\
B\&H & Becker and Hickl \\
bps & Bites per second \\
Ca2+ & Calcium \\
CCD & Charge-Coupled Device \\
CMOS & Complementary Metal-Oxide Semiconductor \\
cPs & Counts per second \\
CW & Continuous Wave \\
DAQ & Data acquisition \\
DMM & Deformable Membrane Mirror \\
DNA & Deoxyribonucleic acid \\
DOE & Diffractive Optical Element \\
DVI & Digital Visual Interface \\
ECB & Electric Controlled Birefringence \\
EGFP & Enhanced Green Fluorescent Protein \\
FCS & Fluorescence Correlation Spectroscopy \\
FFT & Fast Fourier Transform \\
FIFO & First-in-first-out \\
FLIM & Fluorescence Lifetime Imaging Microscopy \\
FPGA & Field Programmable Gate Array \\
fps & Frames per second \\
FRET & Förster Resonance Energy Transfer \\
FT & Fourier Transform \\
FWHM & Full-width Half Maximum \\
GFP & Green Fluorescent Protein \\
GS & Gerchberg-Saxton \\
HOT & Holographic Optical Tweezers \\
HWP & Half-wave Plate \\
IR & Infrared \\
IRF & Instrument Response Function \\
LCD & Liquid-crystal Display \\
LCOS & Liquid Crystal on Silicon \\
LUT & Look Up Table \\
MM & Multifocal Multiphoton \\
MMM & Multifocal Multiphoton Microscope \\
MPM & Multiphoton Microscopy \\
mTFP1 & Monomeric Teal Fluorescent Protein \\
NA & Numerical Aperture \\
NIR & Near-Infrared \\
OBJ & Objective \\
PCF & Optical Constant Fraction \\
PMT & Personal Computer \\
& Photomultiplier Tube \\
\hline
\end{tabular}




\begin{tabular}{|c|c|}
\hline PSF & Point spread Function \\
\hline RFP & Red Fluorescent Protein \\
\hline RMS & Root-mean squared \\
\hline RNA & Ribonucleic acid \\
\hline ROI & Region of interest \\
\hline RS & Random Search \\
\hline SHG & Second-Harmonic Generation \\
\hline SHWFS & Shack-Hartmann wavefront sensor \\
\hline SLM & Spatial Light Modulator \\
\hline SNR & Signal-to-noise Ratio \\
\hline SPAD & Single photon avalanche diode \\
\hline STED & Stimulated-emission Depletion \\
\hline TAC & Time-to-Amplitude Converter \\
\hline TCSPC & Time-correlated single photon counting \\
\hline TDC & Time-to-Digital Converter \\
\hline TPM & Two-photon Microscopy \\
\hline TTL & Transistor-transistor logic \\
\hline TWS & Tscherning Wavefront Sensor \\
\hline USB & Universal Serial Bus \\
\hline WFS & Wavefront Sensor \\
\hline WGS & Weighted Gerchberg-Saxton \\
\hline YFP & Yellow Fluorescent Protein \\
\hline SYNC & Synchronization \\
\hline$A_{n}^{m}$ & Zernike coefficient amplitude \\
\hline$E_{a}$ & Wave amplitude \\
\hline$E_{m}$ & Zernike derivative matrix \\
\hline$E_{s}$ & Zonal reconstruction sparse matrix \\
\hline$f_{p}$ & Repetition rate \\
\hline$I^{H}$ & Hologram intensity \\
\hline$I^{T}$ & Target intensity \\
\hline$I_{x}$ & Image plane \\
\hline$k_{t}$ & Transfer rate \\
\hline$M_{x}$ & Mirror \\
\hline$n_{a}$ & Photon pair \\
\hline$N_{n}^{m}$ & Normalization factor \\
\hline$N_{s}$ & Square subregion \\
\hline$N_{T O}$ & Overlap corrected total subregions \\
\hline$N_{T P}$ & Total number of subregions \\
\hline$R_{0}$ & Förster radius \\
\hline$R_{n}^{m}$ & Zernike radial polynomial \\
\hline$u^{H}$ & Hologram field \\
\hline$u^{T}$ & Target field \\
\hline$w_{0}$ & Radial beam waist \\
\hline$w_{m}$ & Weight factor \\
\hline
\end{tabular}




\begin{tabular}{|c|c|}
\hline$z_{0}$ & Axial beam waist \\
\hline$\delta_{m 0}$ & Kronecker delta \\
\hline$\kappa^{2}$ & Orientation factor \\
\hline$\tau_{p}$ & Pulse width \\
\hline$\varphi^{H}$ & Hologram plane \\
\hline$\varphi^{T}$ & Target plane \\
\hline$A$ & Area \\
\hline$b$ & Pixel brightness \\
\hline$c$ & Speed of light \\
\hline C & Sparse matrix \\
\hline$d$ & Vertex distance \\
\hline $\mathrm{E}$ & Fret efficiency \\
\hline$f$ & Focal length \\
\hline $\mathcal{F}$ & Fourier transform \\
\hline $\mathrm{h}$ & Plank's constant \\
\hline$\hbar$ & Reduced Plank constant \\
\hline i & Imaginary symbol \\
\hline 1 & Intensity \\
\hline $\mathrm{J}(\lambda)$ & Normalized donor emission spectrum \\
\hline$L$ & Lens \\
\hline$M$ & Magnification \\
\hline$m$ & Zernike azimuthal frequency \\
\hline $\mathrm{n}$ & Refractive index \\
\hline$n$ & Zernike radial order \\
\hline $\mathrm{N}$ & Beamlet number \\
\hline$p$ & Centre-of-mass \\
\hline$P$ & Power \\
\hline$p, \theta$ & Polar coordinates in spatial domain \\
\hline s & Slope \\
\hline$U$ & Uniformity \\
\hline$u$ & unit vector $\mathrm{x}$-direction \\
\hline$v$ & unit vector $y$-direction \\
\hline$w$ & Wavefront \\
\hline$x, y$ & Cartesian coordinates \\
\hline Z & Zernike Polynomials \\
\hline$\alpha$ & Fractional FRET populations \\
\hline$\delta$ & Molecular cross-section \\
\hline$\eta$ & Quantum yield \\
\hline$\theta$ & Angle \\
\hline$\lambda$ & Wavelength \\
\hline$\tau$ & Lifetime \\
\hline$\chi^{2}$ & Chi-Squared \\
\hline$\Omega$ & Separation factor \\
\hline
\end{tabular}




\section{LIST OF FIGURES}

Figure 1: Simplified Jablonski scheme for two-photon excitation. After excitation by two infrared photons (red), the relaxation of the fluorescent molecule to the ground state results in photon emission (green). 28

Figure 2: Principal of TCSPC. The detection of single photons and the measurement of their arrival times over a series of periods (A) results in a histogram (B) of the number over counts over time, characteristic of the fluorophore. 34

Figure 3: Multi-detector and multi-wavelength operation principle. Fluorescence emission is spectrally split onto two separate detectors ( 1 and 2 , represented as red and green respectively), which are routed into a single TAC (A). TCSPC is performed in parallel on both detectors (Channel $1 / 2$ ). The resulting photon counts are saved as different histograms (B). Histograms are the result of numerous counts over time; as explained in Figure 2

Figure 4: Z-stack of multiphoton images of EGFP-CXCR4 in mtln3 tumour grown in the mammary fat pad of a nude mouse. Loss of signal due to scattering, fluorescence absorption and tissue induced aberrations is apparent as a function of depth (Ameer-Beg \& Fruhwirth, unpublished data).

Figure 5: Illustration of the principles of adaptive optics through phase conjugation in microscopy. Laser illumination is vertically descendent and focused in the sample. A-B represents the principle of focusing through a high-NA objective with a refractive index mismatch. C represents focusing through complex specimens that induce aberrations. $D$ the specimen-induced aberrations are cancelled out by a conjugate phase.

Figure 6: Schematic representation of the various Zernike modes $Z(n, m)$, known as the Zernike pyramid (Costa and Ferrer-Blasco, 2010). Subscript $\mathrm{n}$ indicates radial order, which gives the row number in the pyramid. Superscript $m$ indicates azimuthal frequency, which gives the column number in the pyramid. 52 
Figure 7: Shack-Hartmann WFS; on the left a uniform wave without distortions results in a uniform and equally spaced grid of spots on the sensor, on the right a distorted wavefront is incident creating a variation of the spots. Image adapted from Thorlabs.com. .55

Figure 8: Schematic of a TWS used to evaluate distortions reflected from the retina. Laser light incident on the Tscherning screen produces beamlets, which are projected on the retina. The wavefront is calculated from the displacements presented on the CCD camera. Lenses denominated as $L$. Image reproduced from (Rozema et al., 2005).

Figure 9: Zonal reconstruction configurations based on the $x$ and $y$ slope components of the gradient. First square represents the Fried approach, the second the Hugdin and the far right is the Southwell configuration. The horizontal and vertical lines are the $x$ and $y$ component, respectively (Dai, 2008)

Figure 10: Single Photon Avalanche Diode; representation of individual SPAD and circuits (left) and entire 32×32 SPAD array (right)

Figure 11: Photon count comparison between a B\&H PMH-100 and a SPAD in the non-descanned detection. Fluorescence generated by a $10 \mathrm{~mW}$ excitation of a fluorescent block is shown as counts per ms. Error bars calculated as $\mathrm{N}$. 73

Figure 12: Schematic of TWS setup. The array from the sample plane is directly compared to the beamlets from the reference mirror purposely chosen with a high degree of flatness. The laser was expanded via a telescope (focal lengths $50 \mathrm{~mm}$ and $150 \mathrm{~mm}$ ) and projected onto the microlens array. Recaptured by a $150 \mathrm{~mm}$ lens was focused on the sample using an x40 0.9 NA air objective. Retroreflected aberrated beamlets were separated from the incident beamlets via a beam splitter cube and refocused onto a CMOS camera (Thorlabs) by a $200 \mathrm{~mm}$ focal length lens.

Figure 13: Effects of spherical aberration induced by $0.01 \mathrm{~mm}$ spherical aberration on the multifocal array generated via a microlens array in a TWS configuration. An aberration free environment (left) with spherical aberration (right) demonstrates 
high sensitivity to aberrations. Comparative line profile (orange) between nonaberrated (blue curve) and aberrated (red curve) shown below. Scale bar $100 \mu \mathrm{m}$.

Figure 14: Expanded area of the beams in the region indicated by the red square in Figure 13. It is clear that the high sensitivity of the beamlets to a variation in refractive indexes resulted in significant degradation of intensity. The comet tails displayed reduces centre-of-mass accuracy and increases possibility of crosstalk between neighbouring focal spots. Scale bar $50 \mu \mathrm{m}$. Image contrast altered to aid visualization. 78

Figure 15: Spherical aberration coefficient determined vs. the various objective intervals, which provoke the spherical aberrations. In all cases the defocus was set to a minimum before determining the remaining coefficients. 79

Figure 16: Spherical aberration coefficient determined vs. the incremental objective intervals inducing spherical aberrations. In all cases the defocus was set to a minimum before determining the remaining coefficients. As expected, throughout the experiment it was noted that the spherical term gained increased importance.

Figure 17: Interference pattern (left) registered at every iteration of the grey level variation was acquired using a double pinhole calibration setup (right). The pattern demonstrates a linear relationship between $0-2 \pi$ over the $0-255$ grey level range projected on the microdisplay.

Figure 18: The linear relationship between $0-2 \pi$ is due to the gamma curve calibration set on the SLM firmware. 86

Figure 19: Calibrated gamma curve for $800 \mathrm{~nm}$ produced via the checkerboard pattern exemplified in black and green.

Figure 20: Boulder HSPDM512 SLM wavelength calibration using the checkerboard pattern approach. A calibrated gamma curve produces zeroth order spot intensity 
in agreement with $(1+\cos \theta) / 2$, as shown by the images presented. Scale bar 200 $\mu \mathrm{m}$.

Figure 21: Projected Fresnel lenses on the Holoeye Pluto NIR2 SLM microdisplay with the added blaze function ( $\mathrm{A}$ and $\mathrm{C}$ ) and the result at the intermediate focal plane ( $B$ and $D) . A / B$ and $C / D$ represent a $2 \times 2$ and $4 \times 4$ array respectively. Images acquired using a CMOS camera with AO correction for SLM microdisplay. Scale bar $100 \mu \mathrm{m}$.

Figure 22: Schematic of RS optimization adapted for the SLM using Zernike polynomials. The algorithm consists of applying random Zernike coefficient amplitudes and registering the intensity. Coefficients corresponding to improvement are recorded. Once improvements stagnate the solution is found... 91

Figure 23: Laser focal spot signal improvement obtained in the Fourier plane the SLM performed by the RS optimization using Zernike polynomials. The preoptimized $(A)$ is shown in comparison with the post-optimized obtained (B). Despite significant improvement B still shows residual aberrations. Overexposure is deliberate to enhance the asymmetry of low intensity features. 92

Figure 24: Original immediate focal plane of the Fresnel pattern projection (left) demonstrates significant improvement (right) after application of RS-AO pattern (middle) Scale bar $100 \mu \mathrm{m}$.

Figure 25: Fresnel lenses with variable aberration applied. Overexposure is deliberate to enhance the asymmetry of low intensity features. The first spot is the result of system corrections (top left), the second (clockwise) has added spherical aberration, the third has added defocus and astigmatism and the forth was individually optimized for all. Scale bar $100 \mu \mathrm{m}$.

Figure 26: GS algorithm flow chart. An approximation to the desired target intensity results from sequential calculations (steps 2-5, Eq. 39-42) of the phase function, producing a hologram (step 6). The Random phase only takes part of the loop in the first iteration 
Figure 27: Demonstration of the iterative nature of the GS algorithm. Images acquired by Thorlabs CMOS camera in the Fourier plane of the SLM. Scale bar 100 $\mu \mathrm{m}$. The first iteration of the desired pattern shows only the contours of the desired intensity pattern. As the algorithm progresses a more accurate approximate is attained, as the full desired target pattern is evident with a higher intensity. The full 30 iterations have duration of approximately 2 minutes 97

Figure 28: GS phase projection in the Hologram plane and resulting focal plane detected by a CMOS camera. Vertical line profile shows the variations in intensity throughout the beamlet array. Scale bar $100 \mu \mathrm{m}$. 98

Figure 29: Generation of a series of focal volumes captured by the CMOS camera in the focal plane. The total amount of beamlets generated (top to bottom, left to right) $64,256,400$ and 625 . Scale bar $100 \mu \mathrm{m}$. 98

Figure 30: MegaFLI schematic in the descanned configuration. OBJ: objective lens, HWP: half-wave plate. The $200 \mathrm{~mm}$ tube lens is housed in a Nikon Ti-E microscope body and the illumination source is a Coherent Chameleon Vision II. Image planes represented by IX are multifocal excitation (red)/fluorescence (green) planes consequence of the GS algorithm hologram projection on the SLM located in the Fourier plane of L3 (lenses and mirrors denoted Lx and Mx) (Section 11.2)......... 105

Figure 31: Megaframe firmware architecture schematic. Megaframe camera array is split into two $32 \times 16$ sub-arrays of pixels. The data is de-serialized, read out in sequence and data acquired within a user selected 64-pixel ROI is transferred. The TDC timestamps are placed in the FIFO buffer, which is transferred via USB2 to the host PC.

Figure 32: MegaFLI schematic. OBJ: objective lens, HWP: half-wave plate. The $200 \mathrm{~mm}$ tube lens is housed in a Nikon Ti-E microscope body and the illumination source is a Coherent Chameleon Vision II. Image planes represented by IX are multifocal excitation (red)/fluorescence (green) planes consequence of the GS algorithm hologram projection on the SLM located in the Fourier plane of L3 
(lenses and mirrors denoted as Lx and Mx). Relay optics in the excitation between L4 and M1have been omitted. Optical rationale detailed in Section 11.2 108

Figure 33: Multifocal multiphoton image of fixed Convallaria majaralis sample (Leica) in the non-descanned detection path. A $125 \times 125 \mu \mathrm{m}$ area was acquired with a 25 square beamlet array with $800 \mathrm{~nm}$ excitation wavelength and emission filter with $40 \mathrm{~nm}$ bandwidth centred at $520 \mathrm{~nm}$. Individually normalized acquired intensity (left) is merged with the lifetime (middle) to provide a combined image (right) confirming the precise overlap of the lifetime and intensity positions.

Figure 34: Intensity (150x150 pixels) response and line profile of a $3 \times 3$ generated fluorescence scanned over an individual SPAD array.

Figure 35: Multifocal manual alignment setup. OBJ: objective lens, HWP: half-wave plate. The $200 \mathrm{~mm}$ tube lens is housed in a Nikon Ti-E microscope body and the illumination source is a Coherent Chameleon Vision II. Multifocal multiphoton laser light is represented in red and the fluorescence is in green. The CMOS camera is configured in reflectance to monitor the illuminating surface of the SPAD. Reflected imaging performed by a beam splitter cube and focused by a $200 \mathrm{~mm}$ lens. Optical layout detailed in section 3.1.

Figure 36: Comparison of manual (A/B) with automatic alignment procedure (C/D). Manual alignment steps illustrated: Original reflection detected $(A)$ (red and green represent SPAD and fluorescence reflection), rotational correction and minor translational correction and full alignment onto less noisy SPAD region (B). Automatic alignment procedure demonstrated: Original intensities detected (C), centre-of-mass calculation to correct for GS spots positining provides realignment on the detectors (D). Automatic alignement provides a more light efficient (due to abcence of beam splitter) and more accurate alignment procedure. 114

Figure 37: Uniformity comparison chart between GS and weighted GS algorithm for various beamlet arrays generated. Multifocal pattern projected in the column A. The fluorescent intensity of a standard array of beamlets produced (column B) 
shows increased uniformity (column C) once the WGS is implemented. Uniformity presented in accordance to Eq. 46 (column D)

Figure 38: Image alignment and stitching. Original intensity image (left) shows higher than background intensity lines on the border separating detectors consequence of a scanning artefact. Eliminating the duplicated values at the edge of each sub-image reduces image size to $240 \times 240$ pixels. The compensated image is shown on the right. Images have an added false colour LUT to aid visualization.. 119

Figure 39: Typical transient produced by the MM-FLIM system. The LevenbergMarquardt mono-exponential fit is represented and red and the data points in blue.

Figure 40: Residual distribution demonstrates a random spread around zero indicating adequate fitting $\left(\chi^{2}=1.08\right)$.

Figure 41: Lateral (XY, Top Left) and axial (YZ, Top Right) acquisitions of a $100 \mathrm{~nm}$ fluorescent bead (contrast was modified to aid visualization). The respective line profile and Gaussian fit (bottom) confirms diffraction-limited performance in the lateral and axial directions.

Figure 42: Demonstration of intensity (left) and lifetime contrast (right) using a fixed Convallaria Majalis (Leica) sample. The $10 \mu \mathrm{m}$ z-stack was acquired using 25 beamlets in $200 \mathrm{~s}$. Respective $X Z / Y Z$ midway projections at the midway point and 3D reconstruction shown. Scale bar $10 \mu \mathrm{m}$ 125

Figure 43: Multifocal (25 beamlets) fluorescence intensity image of Convallaria majalis taken using $800 \mathrm{~nm}$ excitation using the standard GS algorithm (left) and the WGS with a Red Hot LUT (Image J). As expected, the intensity distribution is more uniform in the WGS. 126

Figure 44: Selective beam scanning employed. The GS algorithm allows for fluorescence to be imaged on a pixel-by-pixel basis and therefore it is possible to quantify the optical crosstalk. The first column shows the selected pattern, the 
intensity and subsequent lifetime calculation is shown in columns 2, 3 respectively. Scale bar $15 \mu \mathrm{m}$

Figure 45: ROI of selective beam scanning (A) with normalized images demonstrate crosstalk (B). Analysis demonstrates a $0.2 \pm 0.1 \%$ optical crosstalk in nonilluminated detectors (C).

Figure 46: Intensity (left) and lifetime (right) of MCF7 cells transfected with GFPCaaX $5 \mu \mathrm{m}$ z-stack acquired using 25 beamlets exhibiting the respective $\mathrm{XZ} / \mathrm{YZ}$ axial projections at the midway point and 3D reconstruction. 3D images of EGFP-CAAX cells acquired using 25 beamlets with $900 \mathrm{~nm}$ excitation demonstrate the expression of EGFP throughout the cell. Scale bar $10 \mu \mathrm{m}$ 130

Figure 47: Fluorescence lifetime distribution of the MCF7 EGFP-CAAX cell. As the cell contains only GFP, the average lifetime value of $2.4 \pm 0.11 \mathrm{~ns}$ is consistent with previously measured datasets.

Figure 48: Intensity, lifetime and composite images of MCF7 cells expressing EGFP connected to mRFP using amino acid chains of various sizes compared with MCF7 cells expressing EGFP alone.

Figure 49: High-speed in-vivo TCSPC MM-FLIM. Intensity (left) and fluorescent lifetime (right) of a 4 day Zebrafish embryo with A-375MM cells expressing a modified Rho-A biosensor injected into the pericardial cavity. The z-stack was produced using 25 beamlets with $900 \mathrm{~nm}$ excitation wavelength at a rate of $10 \mathrm{~s}$ per acquisition. The $62.5 \times 62.5 \times 40 \mu \mathrm{m}$ z-stack exhibits the respective $\mathrm{XZ} / \mathrm{YZ}$ axial projections at the midway point and 3D perspective data. 134

Figure 50: Fluorescence lifetime histogram of the Zebrafish optical stack obtained using a Levenberg-Marquardt mono-exponential fitting. 135

Figure 51: FRET efficiency of the Zebrafish stack. 135

Figure 52: Schematic of illumination procedure using the pupil-segmentation algorithm. The ideal focus translates ( $a$ and $c$ ) to all rays intersecting at the same 
focal spot. Segmentation of the pupil permits analysis of aberrated rays; these translate to a shift of the image plane ( $b$ and $d)$.

Figure 53: Flow Chart for Pupil-Segmentation based on Zonal Reconstruction, in which $\mathrm{N}$ equals the number of masked regions 144

Figure 54: Pupil-Segmentation Setup. A TPM laser source expanded to overfill the SLM located in the Hologram plane, responsible for the optimization routine. The non-relevant orders are filtered out in image plane. The first order is then relayed through Galvanometer scanner. The excitation is focused and subsequent fluorescence captured by a x25 1.1 NA objective and seperated from the NIR/IR excitation by a dichroric filter. Sample fluorescence is the spectrally split onto a pair of Hyprid PMT detectors. Lenses and mirrors denoted as Lx and Mx 148

Figure 55: Checkerboard calibration of the Holoeye NIR2 and gamma curve for 920 $\mathrm{nm}$. 149

Figure 56: Graphical illustration of the stepped overlapping procedure. The black border represents the total pupil. Each square, which represents $1 / 9$ of the pupil area, is colour coded. Stepping the sections over the entire pupil space generates a final overlapped mask in which each colour coded segment contributes equally (right). For illustration purposes, a transparency was set in all the individual regions which when recombined illustrate higher influence depending on the density.... 151

Figure 57: Overlapping regional contributions of the displacement calculation. The stepping procedure generates 4 contributing slopes for the central region (white squares), 2 for the borders excluding the corners (blue) and 1 slope for the corners (black).

Figure 58: Flow chart for far-field simulation developed to validate pupilsegmentation wavefront reconstruction via the RMS

Figure 59: Wavefront reconstruction and Zernike Polynomials fit of a complex wavefront (Original Wavefront - left) to various NSxNS independent or overlapping areas (right). 155 
Figure 60 RMS variation with the various NSxNS Pupil-segmentation wavefront reconstructions

Figure 61: Zernike Polynomial coefficient comparison between $3 \times 3$ stepped overlap and $5 \times 5$ phase reconstruction. Defocus (Z4) was not applied but the reconstruction detects minor coefficient amplitude, consequence of spherical aberration (Z12) coupling.

Figure 62: Original (top left) and system correction (bottom left) lateral projections of a $1 \mu \mathrm{m}$ bead projections with the respective axial projections in XZ. Contrast enhanced to aid visualization.

Figure 63: Comparative lateral (left) and axial (right) profile of a $1 \mu \mathrm{m}$ bead on a fixed coverslip before (black line) and after adaptive optical correction (red). Lateral measurement before and after correction is $1.65 \pm 0.02 \mu \mathrm{m}$ and $1.1 \pm 0.1 \mu \mathrm{m}$ and axial measurement is $4.01 \pm 0.21 \mu \mathrm{m}$ and $2.93 \pm 0.17 \mu \mathrm{m}$, respectively 158

Figure 64: FWHM reduction of a $1 \mu \mathrm{m}$ due to adaptive optical correction 158

Figure 65: Projected Zernike Polynomials for system correction. Tip (Z1), tilt (Z2) and defocus (Z4) were calculated in the coefficient analysis but removed in the the reconstruction

Figure 66: System correction applied to Convallaria Majalis (excitation $800 \mathrm{~nm}$ ). Diagonal line profile in (orange) demonstrated in Figure 67. 159

Figure 67: Diagonal line profile of Convallaria Majalis (Figure 66) with system corrections shows minor improvements. 160

Figure 68: Sample correction before (left) and after (right) the pupil-segmentation optimization procedure. Zernike polynomial fitted wavefront applied (middle). Scale bar $30 \mu \mathrm{m}$ (white), line profile for comparative purposes $128 \mu \mathrm{m}$ (orange). 161

Figure 69: Comparative intensity line profile (orange line) before and after sample correction. 
Figure 70: Sample Zernike polynomials coefficients calculated from the $3 \times 3$ stepped overlap procedure. Tip (Z1), tilt (Z2) and defocus (Z4) were calculated in the coefficient analysis but removed in the reconstruction 162

Figure 71: Comparison between coefficients projected for the system and sample Zernike polynomials. Tip (Z1), tilt (Z2) were removed and defocus (Z4) set to zero as these do not contribute to the axial PSF improvement.

Figure 72: Intensity and lifetime distribution within the FOV before (left column) and after adaptive optical correction (right column).

Figure 73: Normalized lifetime distribution. 164

Figure 74: Intensity improvement using $2 \mu \mathrm{m}$ beads located on top a Zebrafish sample $(300 \mu \mathrm{m})$. Scale bar $10 \mu \mathrm{m}$. 165

Figure 75: Lateral line profile of the bead located on top of the Zebrafish sample (Figure 74) before (red) and after (blue) AO correction. 165

Figure 76: Zernike polynomial coefficients determined to correct for the Zebrafish distortions with respect to the reference bead. Tip (Z1), tilt (Z2) were removed and defocus (Z4) set to zero as these do not contribute to the axial PSF improvement. 166

Figure 77: Comparison between coefficients projected for the system and sample Zernike polynomials for Zebrafish sample. Tip (Z1), tilt (Z2) were removed and defocus (Z4) set to zero as these do not contribute to the axial PSF improvement. 166

Figure 78: Zebrafish SHG Intensity and lifetime distribution before (left column) and after adaptive optical correction (right column). Scale bar $30 \mu \mathrm{m}$. 167

Figure 79: Optical section of Zebrafish at a $30 \mu \mathrm{m}$ depth. Intensity and lifetime distribution before (left column) and after adaptive optical correction (right column) Scale bar $100 \mu \mathrm{m}$, line profile $450 \mu \mathrm{m}$ (orange). 168 
Figure 80: Comparative line profile (orange line - Figure 79) before (blue) and after (red) correction.

Figure 81: Intensity (left) and FLIM (right) measurements of MIFT-A GFP tagged distribution in a 3 day old Zebrafish accompanied by the Fluorescence Lifetime Histogram below.

Figure 82: Intensity (left) and FLIM (right) measurements of MIFT-A GFP tagged distribution in a 5 day old Zebrafish accompanied by the Fluorescence Lifetime Histogram below. 180

Figure 83: Temporal filtering of the autofluorescence. Intensity image acquired (left), FLIM image acquired (middle) and autofluorescence removed using temporal filtering (right). Fluorescent lifetime histograms over the full range and the temporally filtered range demonstrated below. 181

\section{LIST OF TABLES}

Table 1: Comparative chart of various FLIM techniques (Suhling et al., 2005). 40

Table 2: Mathematical representation and meaning of various Zernike modes where $\theta$ and $p$ are the radial and angular coordinates. The lower order Zernike modes represent a range of known aberrations and the higher orders more complex aberrations. Spherical aberrations are of particular interest as these represent the Zernike circle polynomials of higher even $\mathrm{n}$ order and zero kind: aberrations with no azimuthal variation.

Table 3: Comparison of various SPADs (Villa et al., 2012).

Table 4: Comparison between various commercially available reflective phase only SLMs

Table 5: FRET efficiencies registered for various amino acid chain lengths 132 


\section{THESIS OVERVIEW}

In order to enhance the performance of multiphoton fluorescence lifetime imaging (FLIM) systems for in vivo applications it is key to improve: acquisition speed and imaging resolution caused by refractive index heterogeneities. Results presented address both of these issues in isolation; having developed a multifocal multiphoton (MM) FLIM system and a single beam scanning multiphoton FLIM pupil segmentation adaptive optics platform.

The introduction (Chapter 1) concentrates on optical methods developed in the context of multiphoton microscopy (MPM) or more specifically two-photon microscopy (TPM) (Denk et al., 1990). TPM enables long-term imaging of in vivo biological specimens, image generation at increased tissue depth, and higher signalto-noise images compared to wide-field and confocal approaches (Helmchen and Denk, 2005). To obtain insight regarding biological interactions the excitation method has been coupled with FLIM. Multiphoton time-correlated single photon counting (TCSPC) FLIM is a method for obtaining functional, high-resolution images of biological structures (Festy et al., 2007; Fruhwirth et al., 2011; Suhling et al., 2005). The high temporal resolution offered is fundamentally limited by Poisson noise such that photon counts in the thousands or tens of thousands are required to achieve accurate lifetime estimations (Barber et al., 2009). In laser scanning microscopy, such photon statistics are typically only possible with scanning times between 3-5 minutes (Peter and Ameer - Beg, 2004) due to limitations in overall count rate of 1 Mega counts per second (Mcps). In this thesis, two methods are investigated; multifocal or parallel beam excitation and adaptive optics, to counteract some of the fundamental limitations of MPM-TCSPC-FLIM.

Chapter 2 of the thesis specifically concentrates on the development of MM-FLIM microscope, called MegaFLI. The objective of this chapter is to describe the development of the MM-FLIM system. In the context of this thesis, multifocal imaging consists of producing a series of parallel excitation volumes in the focal plane and re-imaging the light from that volume onto a detector (whether that is retroreflected excitation, fluorescence or a harmonic due to non-linear frequency 
mixing). Simplistically, potential data acquisition rates must increase proportionally to the number of beamlets produced using parallel detection. The imaging system (and throughout this thesis), a spatial light modulator (SLM) is employed to produce a phase hologram, which is projected onto the backfocal plane of an objective generating beamlets in the focal (object) plane. The fluorescence emitted is then reimaged onto a novel single-photon avalanche diode (SPAD) array, capable of parallelized TCSPC. The multifocal excitation design achieved in conjunction with the multi-detection architecture, presents significant improvement over existing TCSPC systems, opening the way to truly high-throughput FLIM (Coelho et al., 2013; Li et al., 2012a; Poland et al., 2013). Implementation of MM imaging, exemplar imaging results and future prospects are discussed.

Chapter 3 specifically focuses on adaptive optics in the context of multiphoton imaging for in vivo fluorescence lifetime imaging applications. Adaptive optics, as a technique, is principally concerned with counteracting refractive index mismatches, responsible for signal and resolution degradation as a function of depth (Booth, 2007). We investigated the adaptive pupil segmentation methodology originally proposed by Betzig and co-workers (Ji et al., 2010). Modelled as phase variations in the pupil of the objective lens, reconstruction of the wavefront is calculated by pupil segmentation. In brief, fluorescence (or scattered light) collected from the focal plane permits modelling of the aberrated rays generated from an excitation subregion. The chapter describes the design of the adaptive optical system with the objective of improving signal-to-noise for the acquisition of FLIM. Results achieved using modelling, sample aberration characterization and exemplar projects are presented with adaptive optic correction showing signal and resolution improvement in live zebrafish and oncological histological sections with FLIM. 


\section{CHAPTER 1- INTRODUCTION}

Optical microscopy has seen a vast development since Jansen's first in 1595 (Ball, 1966). Abbe's 1873 interpretation of image formation led to the laws governing diffraction theory, a milestone in microscopy (Abbe, 1873). The Abbe limit corresponds to the distance of the finest periodic structure, which can be imaged by an optical microscope. In combination with Carl Zeiss, Ernest Abbe pioneered manufacturing of optical microscopes (Konig and Halbhuber, 1998). More recent advances in digital imaging, instrumentation, analysis and fluorescent labels permit quantitative measurements as modern optical microscopy has become a key method of visualizing structure in living cells and organisms at submicron resolution (Amos, 2000; Dyba et al., 2003; Lakowicz, 2006). The following sections concentrate on modern technological advancements for the imaging of biological entities.

\section{MULTIPHOTON MICROSCOPY}

In 1931, Maria Göppert-Mayer theoretically predicted that two photons of lesser energy arriving simultaneously can generate an excitation equivalent of that produced by the absorption of a single photon of higher energy in a process named multiphoton or two-photon excitation (Göppert-Mayer, 1931). However, it was not until the invention of the laser that this was demonstrated experimentally (Peticolas et al., 1963). More recently, Denk et al. (Science 1990) showed that twophoton excitation could be used practically in microscopy, in a unique form of laser scanning microscopy. For practical purposes, photons are chosen such that excitation wavelengths $\left(\lambda_{T P M}\right)$ are approximately double those of single photon excitation $\left(\lambda_{1 P}\right)\left(\lambda_{T P M} \approx 2 \lambda_{1 P}\right)$ (So et al., 2000) (Figure 1). Whilst it is convenient to have both infrared (IR) photons for a single excitation source, there are advantages to the more general sum frequency absorption case (Campagnola and Loew, 2003; Campagnola et al., 1999) such as simultaneous two-photon excitation of three 
chromophores, recently demonstrated elegantly by Beaurepaire and co-workers (Mahou et al., 2012).

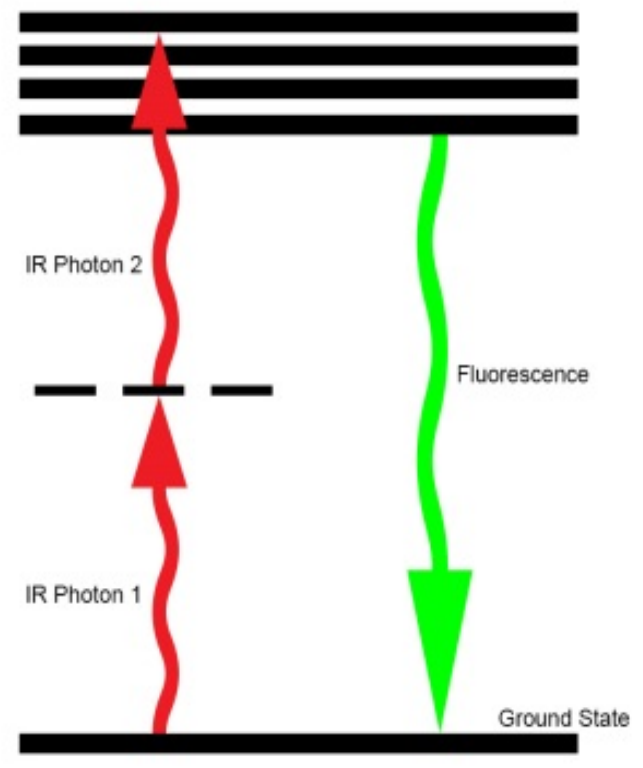

Figure 1: Simplified Jablonski scheme for two-photon excitation. After excitation by two infrared photons (red), the relaxation of the fluorescent molecule to the ground state results in photon emission (green).

In comparison to confocal microscopy, two-photon microscopy (TPM) offers considerable benefits since excitation is confined to a femtolitre volume in the vicinity of the focal plane, reducing overall photobleaching and phototoxicity of thick samples (Centonze and White, 1998; Denk et al., 1990; Helmchen and Denk, 2005; Williams et al., 2001). Out-of-focus excitation is avoided due to the localization of the excitation making the confocal pinhole obsolete (Amos and White, 2003; Yuste and Denk, 1995). Non-linear excitation also results in significant improvement in optical penetration since IR light penetrates tissue more effectively than visible excitation wavelengths due to the wavelength dependence of Rayleigh scattering and low linear absorption (Cahalan et al., 2002; Denk and Svoboda, 1997; Konig, 2000; Williams et al., 2001). The absorption spectra of major intracellular absorbers have shown an optical window in the IR and near infrared (NIR) excitation range (Konig, 2000; Konig et al., 1997). TPM is applicable to thick, turbid samples and also simplifies the collection optics scheme (Diaspro et al., 2006; Dong 
et al., 2003). The observation time for a fluorophore, excited via two-photon absorption, decreases with the third power of the excitation intensity due to photobleaching in the focal plane. At the lowest excitation intensities in the focal plane, photobleaching rates between one- and two-photon excitation are comparable, but at power levels that are typically used in biological imaging (3-5 $\mathrm{mW}$ at the sample for $150 \mathrm{fs}$ laser pulses) this difference has been shown to escalate by a factor of 10 (Diaspro et al., 2005; Patterson and Piston, 2000). Since the photobleaching process is principally via the triplet state, differences in the excitation scheme (for example to higher singlet states where singlet-triplet coupling is more likely) between linear and non-linear excitation are the primary mechanism by which these differences occur (Patterson and Piston, 2000).

The use of longer excitation wavelengths for TPM results in a nominal deterioration of resolution compared to theoretical confocal limits (at which the scattering of visible light is negligible), although the overall improvement in signal-to-noise ratio (SNR) due to the longer scattering length of IR light makes TPM mode applicable at greater depths (Esposito et al., 2004; So et al., 2000).

Optical sectioning properties, as a consequence of the femtolitre excitation volume permits the acquisition of three dimensional data sets (stacks) while providing noticeable SNR improvement. The excitation point spread function (PSF) can be approximated to Gaussian spatial profiles with widths $w_{0}$ and $z_{0}$ in the radial and axial dimensions respectively. Radial and axial beam waist values can be determined by Eq.1 and Eq. 2, respectively (Zipfel et al., 2003):

$$
\begin{gathered}
w_{0} \approx 0.325 \frac{\lambda}{\sqrt{2} N A^{0.91}} \\
z_{0}=\frac{0.532 \lambda}{\sqrt{2}}\left[\frac{1}{n-\sqrt{n^{2}-N A^{2}}}\right]
\end{gathered}
$$

Where $N A$ is the numerical aperture of the objective, $\mathrm{n}$ is the refractive index and $\lambda$ the wavelength of excitation. The full-width half maximum (FWHM), common measurement of optical resolution is obtained by multiplying the expression by $2 \sqrt{\ln 2}$. As determined by the Heisenberg uncertainty principle, the time-scale ( $\tau_{\text {TPM }}$ ) of a two-photon absorption process is (Eq. 3, Eq. 4): 


$$
\tau_{T P M} \approx 10^{-16} \Leftrightarrow 10^{-17}(s) \quad 3
$$

In which;

$$
\tau_{T P M} \approx \frac{\lambda_{1 P}}{4 \pi c} \approx 10^{-16}(s)
$$

With a typical visible wavelength $\lambda_{1 P}=500 \mathrm{~nm}$. In which $c$ is the speed of light in vacuum.

Fluorescence intensity, $I_{f}(t)$, generated via two-photon absorption is proportional to the square of the excitation intensity $I(t)$, the two-photon molecular crosssection $\left(\delta_{2}\right)$ and to the quantum yield $(\eta)$. Excitation intensity in photons. $\mathrm{cm}^{2} \mathrm{~s}^{-1}$ is given by Eq. 5 (Diaspro et al., 2005):

$$
I(t)=\frac{\lambda P(t)}{h c A} \quad 5
$$

Where $h$ is Planck's constant, $P(t)$ is the instantaneous power on the illuminated area, $A=\frac{1}{\pi}\left(\frac{\lambda}{N A}\right)^{2}$, assuming the paraxial approximation. The TPM fluorescence is given by (Eq. 6) (Diaspro et al., 2005) :

$$
I_{f}(t)=\delta_{2} \eta I(t)^{2}=\delta_{2} \eta P(t)^{2}\left(\frac{(N A)^{2}}{2 \hbar c \lambda}\right)^{2}
$$

In which $\hbar$ is the reduced Plank's constant $\left(\hbar=\frac{h}{2 \pi}\right)$. As the molecular crosssections, $\delta_{2}$, for the two-photon absorption is of the order of $10^{-58} \mathrm{~m}^{4} \mathrm{~s}^{-1}$ (Diaspro et al., 2005), it is necessary to have high photon fluxes $\approx$ $10^{30}$ photons $m^{-2} s^{-1}$. This can be reached either by means of high-power continuous wave (CW) lasers or by means of short-pulse lasers, whereby intensity at the sample is greatly enhanced for a short period of time whilst the average power remains low, permitting thermal load reduction to the sample.

Whilst CW-IR or picosecond excitation has been successfully performed, a significant increase of the thermal load on the sample is self-evidently incompatible with life - certainly in the case of CW beams (Bradley et al., 1972; Xu and Webb, 1996). In the case of a femtosecond pulsed laser source with repetition rate $f_{P}$, and pulse width, $\tau_{P}$, the average power $\left(P_{\text {ave }}\right)$ depends on the peak power $\left(P_{\text {peak }}\right)$ as 
$P_{\text {ave }}=\tau_{P} f_{P} P_{\text {peak }}(t)$. The fluorescence intensity per unit time becomes (Eq. 7) (Diaspro et al., 2005):

$$
\left\langle I_{f, P}\right\rangle=\delta_{2} \eta \frac{P_{a v e}^{2}}{\tau_{P} f_{P}}\left(\frac{N A^{2}}{2 \hbar c \lambda}\right)^{2}
$$

$\mathrm{CW}$ and pulsed sources provide the same excitation efficiency in terms of collectable fluorescence intensity assuming that the two-photon excitation crosssection is constant and that the CW laser power is higher by a factor of $\frac{1}{\sqrt{\tau_{P} f_{P}}}$. Therefore $300 \mathrm{~mW}$ delivered by a CW laser is almost equivalent to $1 \mathrm{~mW}$ of a pulsed laser with repetition rate $80 \mathrm{MHz}$ and pulse width $100 \mathrm{fs}$.

The number of photon pairs, $n_{a}$ that fluorescent molecules can absorb per pulse is given as (Eq.8):

$$
n_{a}=\delta_{2} \frac{P_{a v e}^{2}}{\tau_{P} f_{P}{ }^{2}}\left(\frac{N A^{2}}{2 \hbar c \lambda}\right)^{2} \quad 8
$$

Variations of the equations 5-8, may include extra factors which account for the dye concentration (Esposito et al., 2004) and the excitation pulse shape (1.0, 0.66 and 0.59 for Rectangular, Gaussian or Hyperbolic-secant-squared pulse shape respectively) (Diaspro et al., 2005).

Ground state depletion occurs when:

$$
n_{a} f_{P} \approx \frac{1}{\tau}, \text { or } n_{a} f_{P} \tau \approx 1
$$

In which $\tau$ corresponds to the fluorescence lifetime (section 6). Assuming a priori one photon pair per pulse, $n_{a} \approx 1$, with the intensity below the saturation threshold, the pulse repetition rate should be of the order of $\frac{1}{\tau}$ or less. Therefore lifetimes of under $10 \mathrm{~ns}$ correspond to pulse repetitions in the region of $100 \mathrm{MHz}$ which matches the cavity range of Ti:Sapphire laser (Helmchen and Denk, 2005). Most common fluorophores have a fluorescence lifetime in the range 0.5-6 ns (Berezin and Achilefu, 2010). 


\section{KEY COMPONENTS OF A MULTIPHOTON SYSTEM}

In order to successfully perform TPM, basic system components need to be present. This section is a summary of the key components of a multiphoton system such as the laser source (Section 2.1), the scanning system (resonant and non-resonant scanners) (Section 2.2) and detection (descanned and non-descanned detection) (Section 2.3).

\subsection{LASER SOURCE}

A multiphoton microscope requires a pulsed laser source. The most commonly used is the Ti:Sapphire oscillator. The large tuning range $(670-1050 \mathrm{~nm})$ and high power $(>1 \mathrm{~W})$ permit the excitation of a large range of fluorophores. Compact commercially available Ti:Sapphire laser are available providing simple key-turn operation with software control (Helmchen and Denk, 2005).

\subsection{SCANNING SYSTEM}

There are two most common scanning systems; resonant and non-resonant scanners. The usual scanning method is the non-resonant point scanner, which scans the sample as a point in the XY directions. Two mirrors placed perpendicular rotate laterally to permit one mirror to scan the respective axis. In order to ensure the scanned region is linear, a raster scan is implemented and tuned to minimise fly-back of the scan mirror (Xi et al., 2011). In order to compensate for the residual non-linearity, the scan is limited to the linear region of the mirror's motion. Nonresonant scanning permits arbitrary scan directions (line scan, spiral scan etc.), variable scan speed, flexible pixel dwell time and the selection of a scanning area within the field of view (Pawley, 2006). Resonant scanners are based on the resonant scan mirror that oscillates at a fixed frequency, allowing for fast (videorate) scans (Fan et al., 1999). Due to the sinusoidal motion of the scanners, it is necessary to either correct spatial position in software or restrict the scan to the almost linear part of the sine wave. In practice, bi-directional scanning is implemented to avoid loss of the fly-back retrace time (Cheng et al., 2011). Resonant scanners are suitable for applications that benefit from high frame rates, 
but do not require arbitrary scan arrangements (Kirkpatrick et al., 2012; Pawley, 2006).

\subsection{DETECTION}

The most collection efficient detection scheme is the non-descanned detection scheme (Helmchen and Denk, 2005). This consists of splitting the emission with a dichroic filter immediately after the objective. Reimaging the pupil of the objective onto a large area detector then enables efficient imaging of all fluorescence emitted without movement of a scanned point. This is an important issue, since some photomultiplier tubes have spatially sensitive detection (Hakamata, 2006). For single cell imaging, there is no significant advantage between descanned and non-descanned multiphoton when balanced against the increased background noise due to ambient light (Centonze and White, 1998). Non-descanned detection is advantageous in the presence of significant scattering. Furthermore, descanned confocal was shown by Centonze and White to give $>1.5 x$ signal improvement over confocal microscopy (using a non-optimised confocal/multiphoton system). By contrast, descanned detection consists of removing the fluorescence from the optical train after the scanning system. Placing the detector (and dichroic) after the scanning system permits stationary fluorescence on the detector and pinhole (if used) (Gauderon et al., 1999). The detector typically consists of a photon-multiplier tube (PMT), a charge-coupled device (CCD) or avalanche photon diode (APD) (Suhling et al., 2005).

\section{TIME CORRELATED SINGLE PHOTON COUNTING}

Time Correlated Single Photon Counting (TCSPC) is based on high accuracy repetitive timed registration of single photons, emitted by the fluorophore, with respect to a reference (i.e. laser repetition rate) (Lakowicz, 2006). Provided that the probability of registering more than one photon per cycle is low, an adequate histogram of the photon arrivals is formed over a series of excitation cycles (Figure 2). Despite significant improvement in temporal resolution, in comparison with 
other methods (Agronskaia et al., 2003; Levitt et al., 2009), statistical accuracy is limited by photon statistics $(S N R=\sqrt{N}$, where $N$ is the number of photons collected over the acquisition time). For a single beam scanning system with a count rate limited by the sample, rather than detection electronics, high SNR can only be achieved in conjunction with a relatively long scanning time (typically between 3-5 minutes) (Becker, 2008; Becker, 2005).

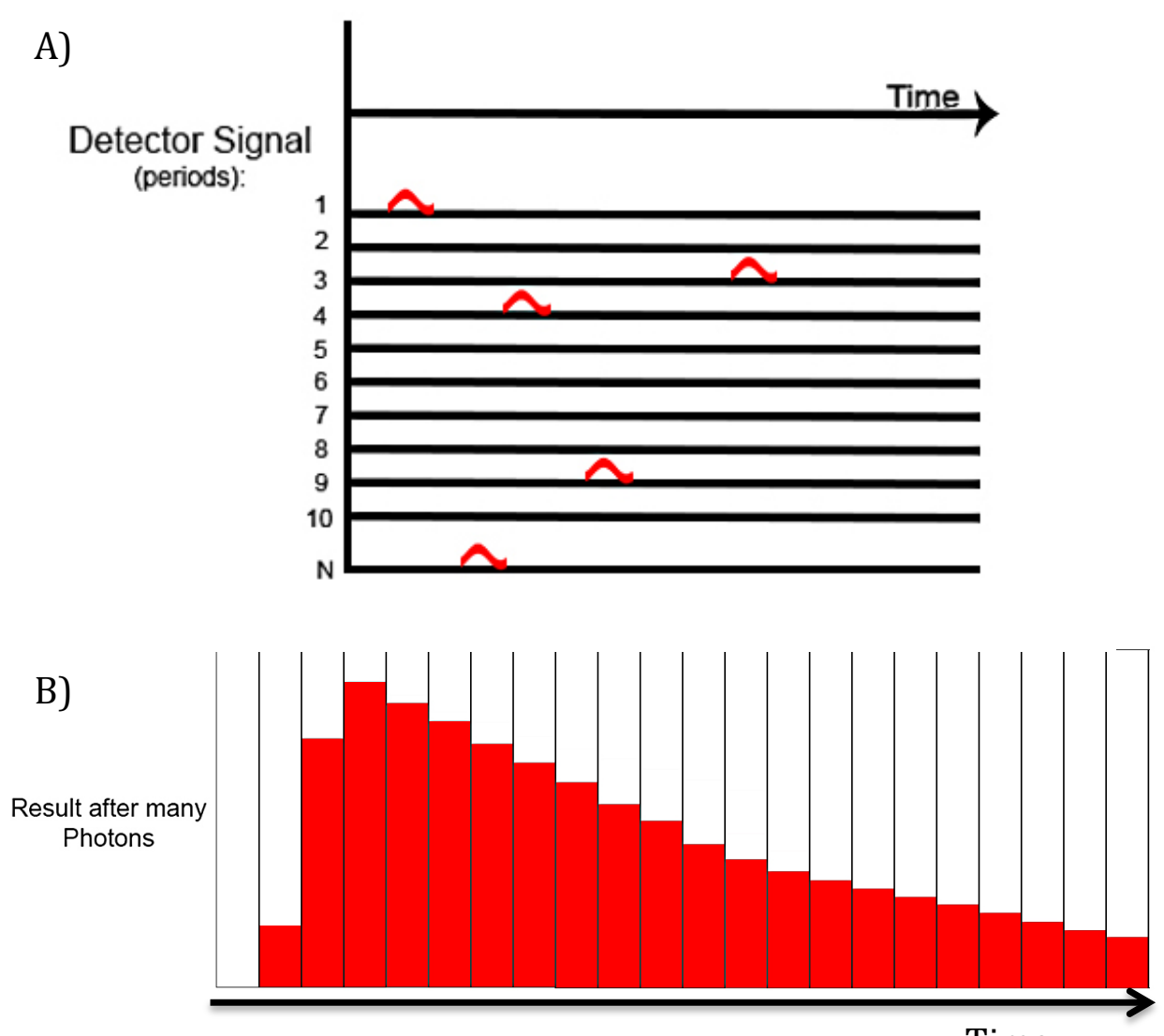

Time

Figure 2: Principal of TCSPC. The detection of single photons and the measurement of their arrival times over a series of periods $(A)$ results in a histogram (B) of the number over counts over time, characteristic of the fluorophore.

The measurement principle for TCSPC is shown in Figure 2. In brief, a pulse of light (reference pulse) excites a molecule, which relaxes to the ground state via emission of a fluorescence photon. The time delay between the excitation reference and fluorescence photon is recorded and the experiment repeated numerous times. The resulting histogram of time-delays is the probability distribution of the excited 
state decay characterised by the excited state lifetime (the time taken for the excited state population to decay to $1 /$ e of its initial value). In reality, since the time decays between excitation and emission are on the order of nanoseconds, the measurement of time must be achieved with picosecond precision. Determination of each photon's arrival time is accomplished using a time-to-amplitude converter (TAC) or time-to-digital converter (TDC). The TAC is often the limiting factor in detection count rate, restricting the maximum count rate to about $5 \times 10^{6}$ photons per second, with dead times after each photon count of approximately $125 \mathrm{~ns}$ due to the TAC reset time (to avoid oscillations) (Becker, 2008; Becker et al., 2004; Duncan et al., 2004). For digital timing circuits this is considerably less of an issue with detector arrangements demonstrating $80 \mathrm{MHz}$ count rate (Rinnenthal et al., 2013). The fundamental limit for TCSPC speed is not, however, the electronics. When we consider the process by which photons are correlated, only the first event on the detector can start the clock. Subsequent events during an excitation cycle will be lost (counting loss). Furthermore, if the probability of a second photon occurring during the excitation is high, the probability distribution of events will be skewed towards shorter times (Sun et al., 2011). This is described as pulse-pile up, which is not to be confused with detector pile-up, which occurs at much higher count rates (Becker, 2005). In order to avoid pulse pile-up, it is important to ensure that much less than one single photon event per pulse is detected. Approximately 0.01 emission events per excitation are generally considered acceptable.

There are two different operating modes in TCSPC applications: "forward mode" characterized by a pulse from an excitation light source to the detection of a photon and "reversed start-stop mode" where the TAC is started with a signal from the detector and stopped with the next reference pulse from the light source. Forward mode is considered to be the classical TCSPC operation mode but with high repetition rate light sources (50 to $100 \mathrm{MHz}$ ) becomes impractical as the TAC must be reset every 10 to 20 ns (125 ns in B\&H hardware). In reversed mode the TAC operates at the rate of the photon detection events as opposed to the much higher excitation rate, desirable to avoid counting losses. Based on the assumption that 
the period of the excitation pulses is constant and free of jitter the TAC output voltage decreases for increasing arrival times of the photons (Becker, 2008).

\section{MULTI-DETECTOR AND MULTI-WAVELENGTH}

Multi-detector and multi-wavelength TCSPC can be performed when photons are split spectrally and projected onto separate detectors (Figure 3). Working under the principle that the detection rate of several photons per period remains an unlikely event, several detectors can be routed and measured using a single TAC. The data from the various detectors is then saved respectively into alterative waveform memory sections using a routing code. However, current commercial technology based on this approach limits the amount of detectors that can be routed into a single TAC, ultimately hindering TCSPC multifocal excitation (Becker, 2008). Alternative multi-detection architectures based on CMOS-SPAD technology have recently shown 1024 parallel TCSPC detection channels, with individual time-todigital converters (Li et al., 2010; Richardson et al., 2009b). 
A)

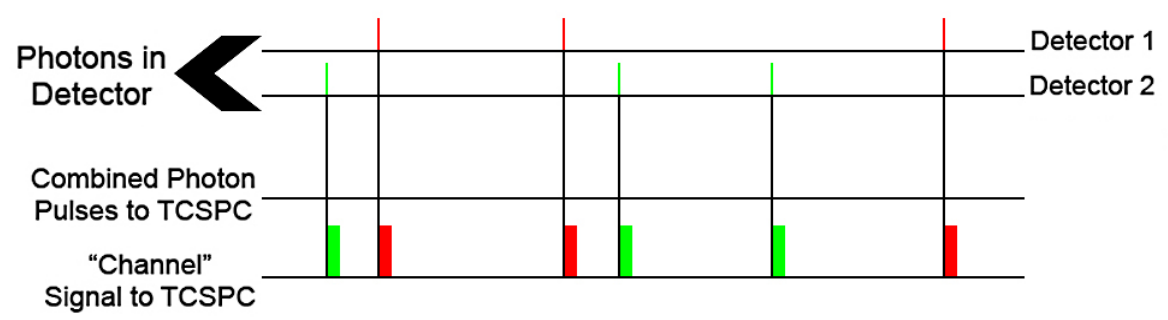

Photons with Channel $=2$

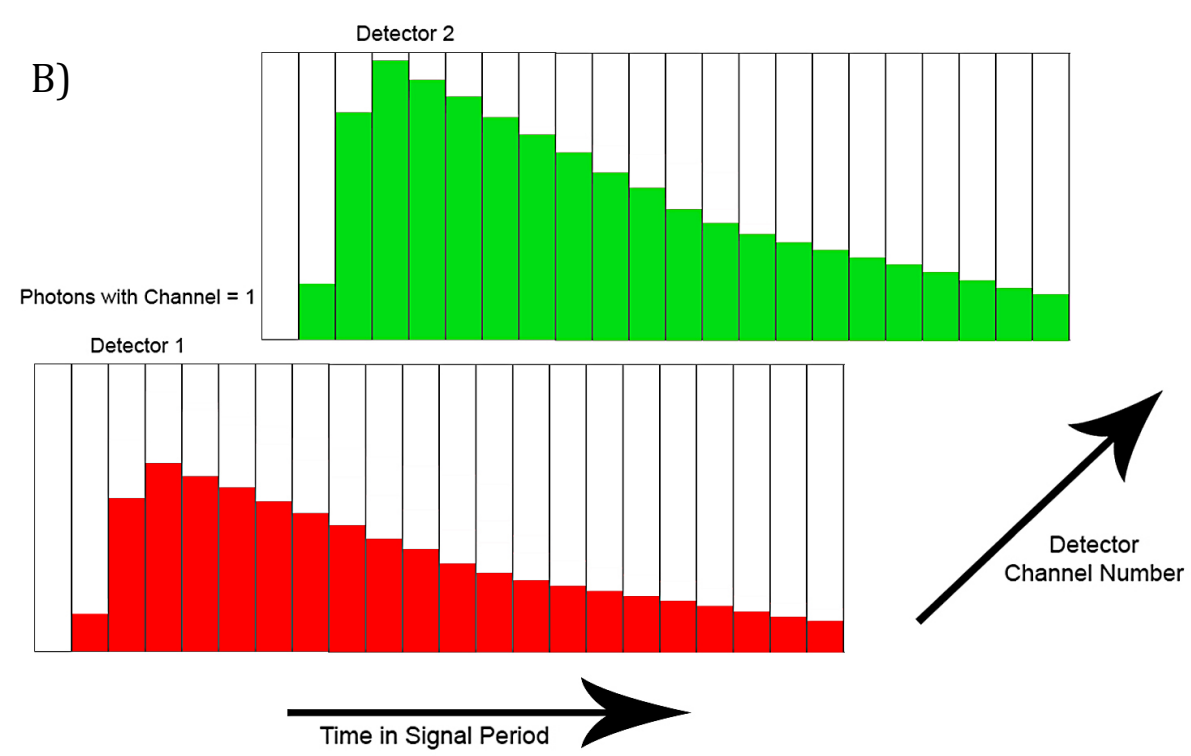

Figure 3: Multi-detector and multi-wavelength operation principle. Fluorescence emission is spectrally split onto two separate detectors ( 1 and 2 , represented as red and green respectively), which are routed into a single TAC (A). TCSPC is performed in parallel on both detectors (Channel 1/2). The resulting photon counts are saved as different histograms (B). Histograms are the result of numerous counts over time; as explained in Figure 2.

\section{TCSPC-FLIM}

TPM excitation combined with TCSPC has been extensively exploited to obtain information on high-resolution images of biological structures and fundamental processes (Becker, 2005; Lakowicz, 2006; Peter and Ameer - Beg, 2004; Suhling et al., 2005; Sun et al., 2011). A particularly interesting application occurs when this excitation method is coupled with FLIM analysis, which can provide information of 
variations in the local molecular environment of fluorophores. The fluorescence lifetime is an intrinsic property of a fluorophore, which corresponds to the average time that a molecule spends in the excited state before returning to the ground state, typically with the emission of a photon (Becker et al., 2004; Duncan et al., 2004; Lakowicz, 2006; Peter et al., 2005; Peter and Ameer - Beg, 2004).

TCSPC FLIM has been widely used to measure populations of interacting protein species on a point-by-point basis with high accuracy (Chen and Periasamy, 2004; Festy et al., 2007; Ganesan et al., 2006; Padilla-Parra et al., 2009; Parsons et al., 2005; Peter and Ameer-Beg, 2004; Sun et al., 2011; Wallrabe and Periasamy, 2005). Fluorescence transients provide a method to distinguish both interacting and non-interacting protein populations, which are distinctive in their decay kinetics (Festy et al., 2007; Ng et al., 1999; Wouters et al., 2001). The decay curve acquired at each pixel comprises of photon counts in different time windows relative to the excitation pulse plotted over time (Figure 2). In the case of a single exponential decay, the fluorescence intensity as a function of time is described as (Lakowicz, 2006):

$$
I(t)=I_{0} e^{-\frac{t}{\tau}} \quad 10
$$

In which $I_{0}$ is the initial intensity. The decay to $1 /$ e of its initial value represents the fluorescence lifetime $\tau$. Complex multi-exponential decays result in multiexponential fluorescence decay models. The measured decay trace is the convolution of the fluorescence decay and the instrument response function (IRF) of the FLIM system. Consequently, an iterative re-convolution algorithm is used to estimate the lifetime information from the measured data, given a measured IRF and the choice of exponential model (Barber et al., 2009; Barber et al., 2005; Luchowski et al., 2009; Siegel et al., 2003). In the case of NIR/IR excitation the IRF is measured using a sample that provides second-harmonic generation (SHG) signal such as a gold colloid (Mukhtar et al., 2002; Talbot et al., 2011).

Alternative techniques exist to TCSPC-FLIM, such as wide-field time gated FLIM (Webb et al., 2002) and streak-camera based FLIM (Biskup et al., 2004; Krishnan et 
al., 2003a; Krishnan et al., 2003b). Wide-field time gated FLIM is equivalent to successive acquisitions of the fluorescence emission taken at various nanosecond delays after the excitation. Utilizing a high-speed gated image intensified camera, the pixels are acquired in parallel. The main benefit of this method is the speed at which the 'snapshots' are taken. Fluorescence lifetime images of calcium fluxes have been acquired with frame rates of $100 \mathrm{~Hz}$ (Agronskaia et al., 2003). However the high acquisition rate results in limited temporal resolution ( $\approx 80 \mathrm{ps})$ and timegated approaches cannot provide single photon sensitivity (Dowling et al., 1998; Suhling et al., 2005). Methods that rely on a pulsed laser source for excitation and the fluorescence is sampled at various delays following the excitation pulse, are denominated as time-domain measurements. FLIM can also be conducted using the frequency-domain. Typically done by heterodyning, or modulating the photomultiplier tube (PMT) at a frequency slightly offset from the frequency at which the laser is modulated (Trabesinger et al., 2002). The difference between these two frequencies is then the cross-correlated beat frequency of the output, which is slow enough to be detected by inexpensive electronics. The lifetime values are then represented by the phase shift or modulation of this cross-correlated signal. The drawbacks of frequency-domain measurements consist of low SNR and temporal resolution. Advances in this approach include the digital frequency domain (Colyer et al., 2008) and more intuitive interpretation of the data such as phasor plots (Stringari et al., 2011). The advantages of the various FLIM techniques are summarised in Table 1. 
Table 1: Comparative chart of various FLIM techniques (Suhling et al., 2005).

\begin{tabular}{|c|c|c|}
\hline Technique & Advantages & Disadvantages \\
\hline $\begin{array}{c}\text { Wide-field frequency } \\
\text { domain }\end{array}$ & $\begin{array}{l}\text { Fast } \\
\text { Relatively Inexpensive }\end{array}$ & $\begin{array}{l}\text { Very low signal-to- } \\
\text { noise and temporal } \\
\text { resolution }\end{array}$ \\
\hline $\begin{array}{l}\text { Time-gated wide-field } \\
\text { time domain }\end{array}$ & $\begin{array}{l}\text { Fast } \\
\text { All pixels acquired in parallel }\end{array}$ & $\begin{array}{l}\text { Low sensitivity } \\
\text { Need pulsed laser }\end{array}$ \\
\hline $\begin{array}{l}\text { Confocal/multiphoton } \\
\text { scanning with time- } \\
\text { correlated single } \\
\text { photon counting }\end{array}$ & $\begin{array}{l}\text { Single photon sensitivity } \\
\text { Large dynamic range } \\
\text { Independent of excitation intensity } \\
\text { fluctuations } \\
\text { Good signal to noise ratio }\end{array}$ & $\begin{array}{c}\text { Slow, each photon } \\
\text { has to be timed } \\
\text { individually }\end{array}$ \\
\hline
\end{tabular}




\section{FRET-FLIM}

Cellular biology has considerably employed FLIM as a means of identifying Förster resonance energy transfer (FRET) upon the interaction between suitable stochiometrically labelled specific proteins, lipids, enzymes, DNA and RNA, as well as cleavage of a protein, or conformational changes within a protein (Suhling et al., 2005). FRET is a process in which energy is transferred non-radiatively from a fluorophore in an electronic excited state serving as a donor, to another chromophore or acceptor (Jares-Erijman and Jovin, 2003).

FRET-FLIM techniques permit spatio-temporal monitoring of the molecular and cellular responses to anti-tumour therapeutics biological agents (Fruhwirth et al., 2011; Gadella Jr et al., 1993; Lakowicz et al., 1992a; Lakowicz et al., 1992b; Wouters and Bastiaens, 1999; Wouters et al., 2001). In the presence of non-radiative processes such as FRET, FLIM is used to measure the quenched donor fluorescence lifetime (Bastiaens and Pepperkok, 2000; Gadella Jr et al., 1993; Sahoo, 2011; Sun et al., 2011).

A method of quantifying FRET via FLIM is to calculate the energy transfer efficiency, in terms of $\tau_{D A}$ and $\tau_{D}$. The FRET efficiency (E) is related to the molecular separation of donor and acceptor and the fluorescence lifetime of the interacting fraction by (Eq. 10):

$$
E=1-\frac{\tau_{D A}}{\tau_{D}}
$$

Where $\tau_{D A}$ is the quenched lifetime of the donor and $\tau_{D}$ the donor in the lifetime of the absence of acceptor (Bugiel et al., 1989; Lakowicz, 2006; Wouters and Bastiaens, 1999).

Interacting FRET pairs are dependent on the transfer rate $k_{t}$, which varies inversely with the $6^{\text {th }}$ power of the donor-acceptor separation $\left(r^{6}\right)$ in a range over 1-10 $\mathrm{nm}$. This proximity has shown to constitute a method of probing the biomolecular domains engaged in complex formation and conformational transition, with a spatial resolution far exceeding the inherent diffraction limit of conventional optical 
microscopy (Clegg, 1992; Mayor and Bilgrami, 2007; Piston and Kremers, 2007; Selvin, 1995). The transfer rate depends on three parameters: the overlap integral $J(\lambda)$, which is the overlap of the donor emission and acceptor absorption spectra, the relative-orientation of the donor absorption and acceptor transitions moments $\left(\kappa^{2}\right)$ and the refractive index $n$. The transfer rate is given in Eq. 12.

$$
k_{t}=\frac{1}{\tau_{0}}\left(\frac{R_{0}}{r}\right)^{6}
$$

In which:

$$
R_{0}=c_{0} \kappa^{2} J(\lambda) n^{-4} \eta
$$

Where $\mathrm{c}_{0}=8.8 \times 10^{-28}, \mathrm{R}_{0}$ the Förster radius $\mathrm{nm}$ and $J(\lambda)=10^{17} \int \mathrm{q}_{\mathrm{d}, \lambda} \varepsilon_{\mathrm{a}, \lambda} \lambda^{4} \mathrm{~d} \lambda$ in $\mathrm{nm}^{6} \mathrm{~mol}^{-1}, \mathrm{q}_{\mathrm{d}, \lambda}$ is the normalized donor emission spectrum, quantum yield $\eta, \mathrm{n}$ is the refractive index and the orientation factor $\kappa^{2}$ which is almost universally assigned the value of $\frac{2}{3}$, assuming random isotropic orientation and rapid rotation during the donor excited-state lifetime (Clegg, 1995; Jares-Erijman and Jovin, 2003; Kenworthy, 2001; Selvin, 2000).

The transfer quantum yield generally denoted as the energy transfer efficiency $E$ can be determined using Equation 14:

$$
E=k_{t} \tau=\frac{R_{0}{ }^{6}}{R_{0}{ }^{6}+r^{6}}
$$

The lifetime of a fluorescent molecule is sensitive to its local microenvironment changes in temperature, $\mathrm{pH}$ and ion (e.g., calcium) concentrations; accurately measured using FLIM (Suhling et al., 2005). FLIM has remarkable advantages compared to other methods that are either invasive, such as microelectrodes (Biran et al., 2007) or require a calibration on the specimen, such as ratiometric imaging (Pollok and Heim, 1999). Using donor lifetime, $\tau_{D}$, as a parameter for measuring relative concentrations makes the measurements insensitive to intensity effects 
such as shading in the image, changes in laser intensity or absorption in the specimen (Sanders et al., 1995; Sun et al., 2011). FLIM measurements are also insensitive to fluorophore concentration and photobleaching (Chen et al., 2003; Suhling et al., 2005). Despite the various advantages of FRET-FLIM it is important to ensure that the donor is found in the same microenvironment in both donor-alone control specimens and specimens containing both donor and acceptor fluorophores, as the fluorescence lifetime of a fluorophore can be influenced the microenvironment (Roy et al., 2008; Sun et al., 2011).

Numerous extensive literature reviews provide periodic updates for the rapidly evolving field of fluorophore development, facilitating the choice of fluorophores for various excitation methods (Drobizhev et al., 2011) and applications (Shaner et al., 2004; Shaner et al., 2005), including FRET (Lam et al., 2012). FRET imaging can be used to probe intermolecular distances but also intramolecular distances. In fact, FRET biosensors have been developed to probe molecular environments (Aoki and Matsuda, 2009; Mochizuki et al., 2001). In a biosensor, the donor and acceptor fluorophore are bound to the extremities of proteins of interest, forming unimolecular FRET sensors (Ishido et al., 2011). A conformational change of the fluorescent pair is induced through stimulation, resulting in FRET (Ai et al., 2008; Rizzo et al., 2004). 


\section{ADAPTIVE OPTICS}

Adaptive optics $(A O)$ is concerned with reducing wavefront distortions therefore improving signal-to-noise ratio and resolution. This concept was first put into widespread practice in astronomy (extensively reviewed in Tyson 2010). Light captured by a telescope is distorted due to atmospheric aberrations. A wavefront sensor (WFS) measures the wavefront of the light to be corrected, then a deformable element is manipulated to compensate for the dynamic changes in the optical path removing undesirable distortions (Babcock, 1953; Dainty, 2007; Tyson, 2010).

Phase conjugation is the key concept in adaptive optics (Herrmann, 1977; Pepper, 1986). As the exciting wavefront is distorted, the amount of correction needed is proportional to the negative wavefront itself. Multiplying the field $E_{A} e^{-i \varnothing}$ by its complex conjugate $E_{A} e^{+i \emptyset}$ is termed phase conjugation, in which $E_{A}$ representes the wave amplitude and $\emptyset$ the phase (Tyson, 2010). In practice this is performed using an appropriate correction element to introduce a phase variation such as a SLM or membrane deformable mirror. Therefore aberrations (Figure 4) are compensated by introducing an equal and opposite phase aberration (Figure 5) (Booth, 2007).

In order to appropriately characterize specific aberrations, it is convenient to represent them as a series of orthogonal functions, such as Zernike polynomials (Zernike, 1934). Zernike modes are regularly used due to their mathematical simplicity, added to the fact that low-order Zernike modes correspond closely to traditional aberration terms, such as astigmatism, coma or spherical aberration (Born and Wolf, 1999).

$\mathrm{AO}$ in the context of microscopy has been developed as a method to compensate system and biological sample induced aberrations through refractive index mismatches (Girkin et al., 2009; Marsh et al., 2003; Neil et al., 2000a; Neil et al., 2000b; Schwertner et al., 2007). These distortions are equated to a distortion of the wavefront from the ideal planar or spherical form, resulting in SNR and resolution 
degradation (Figure 4). These aberrations, modelled as phase variations in the pupil of the objective lens, are cancelled by producing a conjugate phase (Figure 5,d).

Various methods for monitoring the wavefront variation in the presence of aberrations have been developed, the most prominent being the Shack-Hartmann wavefront sensor (Dai, 2008) and interferometric sensors (Booth et al., 1998b; Schwertner et al., 2004). These methods require a point-like reference source (Booth et al., 1998a). An alternative approach to direct wavefront sensing in adaptive microscopy primarily consist of iterative approaches such as the HillClimbing algorithm (Marsh et al., 2003), Random Search (RS) algorithm (Wright et al., 2005), microelectromechanical systems (Tang et al., 2012) and pupilsegmentation approach (Ji et al., 2010). In contrast to wavefront sensing, direct sensing maximizes on a given parameter such as position, shape, intensity or sharpness, without the need of a reference.

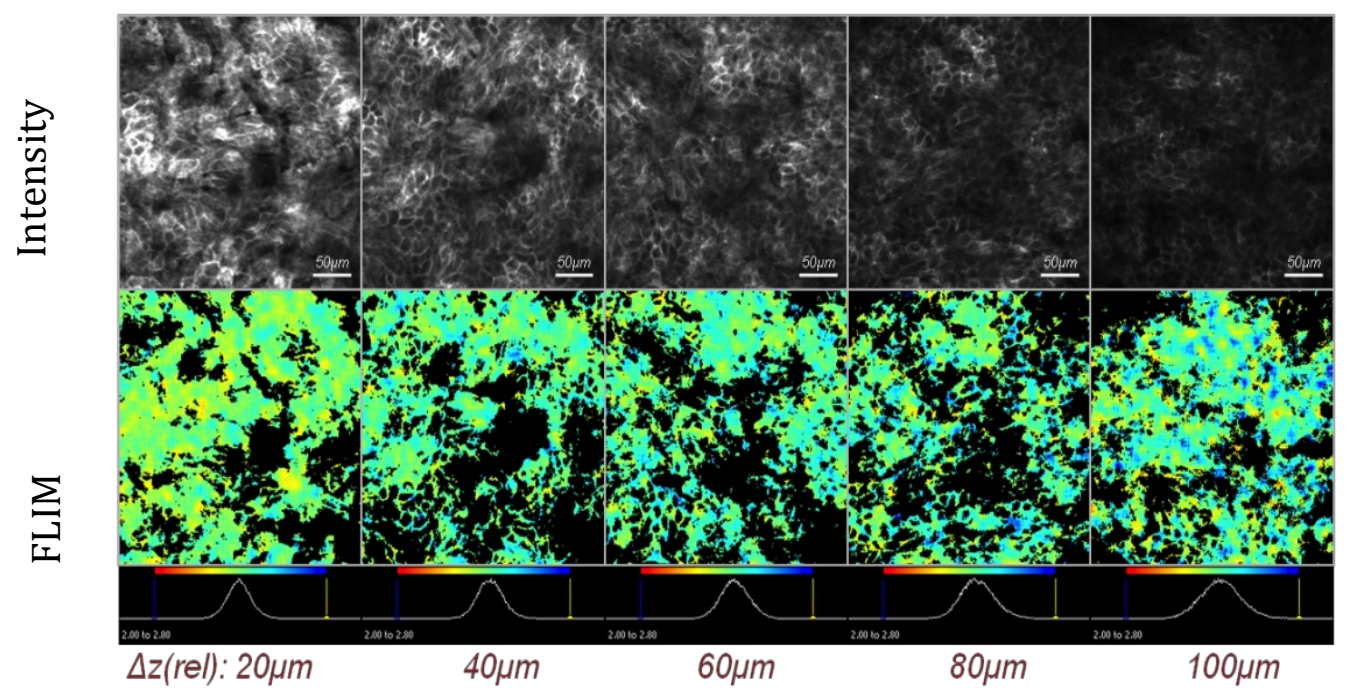

Figure 4: Z-stack of multiphoton images of EGFP-CXCR4 in mtln3 tumour grown in the mammary fat pad of a nude mouse. Loss of signal due to scattering, fluorescence absorption and tissue induced aberrations is apparent as a function of depth (Ameer-Beg \& Fruhwirth, unpublished data). 
a)

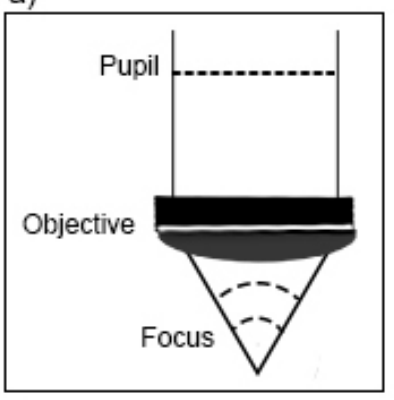

c)

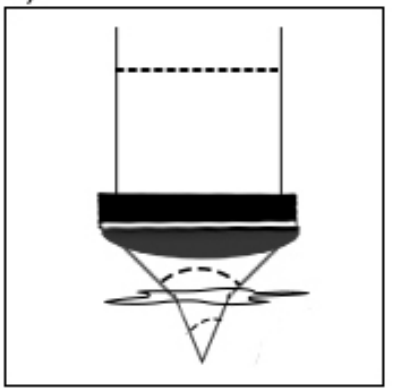

b)

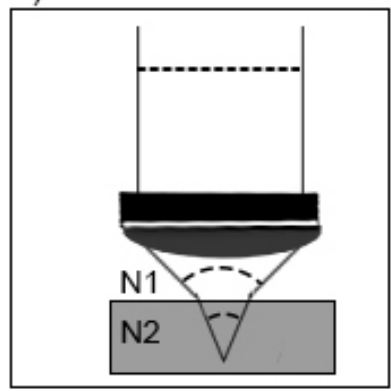

d)

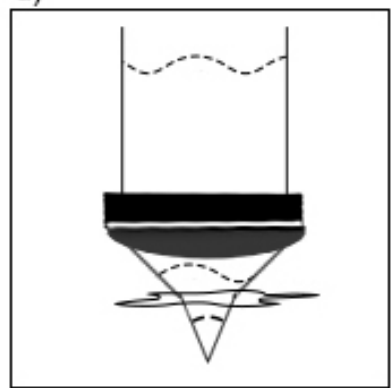

Figure 5: Illustration of the principles of adaptive optics through phase conjugation in microscopy. Laser illumination is vertically descendent and focused in the sample. A-B represents the principle of focusing through a high-NA objective with a refractive index mismatch. C represents focusing through complex specimens that induce aberrations. $D$ the specimen-induced aberrations are cancelled out by a conjugate phase.

\subsection{WAVEFRONT REPRESENTATIONS}

Representing an aberration in a two-dimensional surface can be performed using discrete values that represent the optical path differences at different locations (discrete or zonal wavefront reconstruction - Section 7.3) or analytically by decomposing the aberration into a series of elementary shapes (Analytical or Modal wavefront reconstruction - Section 7.2) (Dainty, 2007; Panagopoulou and Neal, 2004; Tyson, 2010). In the analytical representation, the familiarity of the fundamental components of aberration (defocus, astigmatism, spherical etc.) provides an intuitive representation, as opposed to a topographic view of the discrete values. Once represented, the analytical approach has infinite resolution. As mathematical functions, the value at any point in the two-dimensional surface 
can be accurately determined, unlike the discrete approach for which an interpolation becomes necessary to determine the value between neighbouring points. Therefore computational manipulation of analytical functions is significantly more efficient and straightforward. However, discrete representations provide unique detailed representation of the wavefront at the respective areas resulting in representations with higher spatial frequencies (Carvalho, 2005; Smolek and Klyce, 2003; Yoon et al., 2008). Representing the wavefront as individual tiles segmenting the pupil of the imaging objective reduces over-smoothing (Dai, 2008).

As the analytical reconstruction of the optical surface under-represents a particular sampling distribution with a highly irregular wavefront, techniques have been developed which make use of a combination of discrete and analytical reconstruction. Overlapping regionalized scanning of the sample provides diverse local phase calculations which are then fitted to Zernike polynomials to appropriately characterize the amplitude of the various distortions present (Espinosa et al., 2010).

Regardless of which method is chosen to represent the phase variations, the wavefront, $w$, is related to the corresponding $x y$ components at any given point through the vector gradient equation (Eq.15):

$$
\Delta w=\frac{\delta w}{\delta x} u+\frac{\delta w}{\delta y} v=S^{x} u+S^{y} v
$$

Where $u$ and $v$ are the unit vectors in $x$ and $y$. The objective of either representation is to calculate or reconstruct the wavefront based on the gradient measurements $S^{x}$ and $S^{y}$.

\subsection{ANALYTICAL WAVEFRONT REPRESENTATION}

The analytical wavefront representation method consists of describing the wavefront surface as a summation of smoothly varying modes. Various polynomial representation methods include the Seidel (Wyant and Creath, 1992), Chebyshev (Gil et al., 2007), Laguerre (Tincu, 2010), Taylor (Howland and Howland, 1977), 
Fourier (Dai, 2006) and Zernike (Zernike, 1934). All can be used to describe the wavefront.

In all cases the wavefront, $w$, is decomposed into a set of basic functions $F_{i}$ with an expansion coefficient $a_{i}$ in the form (Eq. 16):

$$
w=\sum_{i=0}^{\infty} a_{i} F_{i} \quad 16
$$

There is no theoretical limitation for the number of functions, which can fit a wavefront. For practical purposes, an upper limit for the summation is replaced by $N$; therefore the expression is truncated by exchanging $\infty$ for $N$.

The representation of $F_{i}$ as orthonormal polynomials provides significant benefits. As the polynomials are orthogonal and normal over a unit area, the truncation of an expansion does not alter the expansion coefficients. All polynomials have a zero mean - the mean value of a wavefront equals the piston coefficient, which translates to a simple offset. The wavefront variance is equal to the sum of the square of each expansion coefficient, which is the basis for the calculation of the root-mean-squared (RMS) wavefront deviation (Dai, 2008).

Due to its mathematical simplicity, modal reconstruction using Zernike modes has become the most common wavefront reconstruction method (Neil et al., 2000b). Zernike polynomials have been extensively applied to compensate for atmospheric turbulence in astronomy (Noll, 1976; Roddier, 1999; Tyson, 2010) and ophthalmological imaging (Godara et al., 2010; Harmening et al., 2007; Porter et al., 2006). Zernike polynomials retain the orthonormality; are related to the classical geometrical aberrations such as the Seidel aberrations; and higher order aberrations balance lower order aberrations to achieve minimum variance.

Zernike polynomial expansion (Eq. 17, Eq. 18) is defined as (Born and Wolf, 1999; Dai, 2008):

$$
\begin{aligned}
& Z_{n}^{m}(p, \theta)=N_{n}^{m} R_{n}^{m}(p) \cos (m \theta) \text { for } m \geq 0,0 \leq p \leq 1,0 \leq \theta \leq 2 \pi \\
& \quad Z_{n}^{m}(p, \theta)=-N_{n}^{m} R_{n}^{m}(p) \sin (m \theta) \text { for } m<0,0 \leq p \leq 1,0 \leq \theta \leq 2 \pi
\end{aligned}
$$


In which,

$$
N_{n}^{m}=\sqrt{\frac{2(n+1)}{1+\delta_{m 0}}} ; \delta_{m 0}=1 \text { for } m=0, \delta_{m 0}=0 \text { for } m \neq 0
$$

Where $N_{n}^{m}$ is the normalization factor (Eq. 19), $R_{n}^{ \pm m}(p)$ (Eq. 23) is the radial component, $\delta_{m 0}$ the Kronecker delta, $n$ is the radial order, $m$ is the azimuthal frequency, $\theta$ and $p$ are the radial and angular coordinates (Bhatia and Wolf, 1954; Born and Wolf, 1999; Mahajan, 1994). A wavefront $w(p, \theta)$ can be represented as a summation of Zernike polynomials (Eq. 20):

$$
w(p, \theta)=\sum A_{n}^{m} Z_{n}^{m}(p, \theta) \quad 20
$$

Where $A_{n}^{m}$ is the coefficient for each Zernike polynomial term, $Z_{n}^{m}(p, \theta)$. The value of the coefficient indicates the amplitude of each Zernike polynomial.

Zernike polynomials have three characteristics that separate them from remaining polynomials. First of all these polynomials have simple rotational symmetry properties that result in a polynomial when expressed in polar coordinates corresponds to (Eq. 21):

$$
V_{n}(p \sin \theta, p \cos \theta)=R(p) G(\theta)
$$

Where $G(\theta)$ is a continuous function that repeats itself every $2 \pi$ radian and satisfies the requirement that rotating the coordinate system by an angle does not change the form of the polynomial (Eq. 22).

$$
G(\theta+\alpha)=G(\theta)+G(\alpha) \quad 22
$$

Where $G(\theta)$ is:

$$
G(\theta)=e^{ \pm i m \theta}
$$

The second property of Zernike polynomials is that the radial function must be a polynomial in $r$ of degree $n$ and contain no power of $r$ less than $m$. The third aspect 
is that the radial component, $R(p)$ must be even if $m$ is even, and odd if $m$ is odd. The radial component, a variant of Jacobi polynomials, is given in Eq. 24.

$$
R_{n}^{ \pm m}(p)=\sum_{k=0}^{\left(\frac{n-m}{2}\right)} \frac{(-1)^{k}(n-k) !}{\left(k ! \frac{(n+m)}{2}-k\right) !\left(k ! \frac{(n-m)}{2}-k\right) !} p^{n-2 k}
$$

The normalization has been selected so that for all valid values of $n$ and $m$,

$$
R_{\bar{n}}^{ \pm m}(1)=1
$$

Each Zernike polynomial represents a specific type of aberration. The Table 2 and Figure 6 below represent the series of Zernike modes with their physical or traditional significance. 


\begin{tabular}{|c|c|c|c|c|}
\hline$n$ & m & $Z_{n}^{m}$ & Meaning & Abbreviation \\
\hline 0 & 0 & 1 & Piston & $Z(0)$ \\
\hline 1 & -1 & $2 p \sin (\theta)$ & Tilt in X-direction (Tip) & $Z(1)$ \\
\hline 1 & 1 & $2 p \cos (\theta)$ & Tilt in Y-direction & $Z(2)$ \\
\hline 2 & -2 & $\sqrt{6} p^{2} \sin (2 \theta)$ & Astigmatism at $\pm 45^{\circ}$ & $Z(3)$ \\
\hline 2 & 0 & $\sqrt{3}\left(2 p^{2}-1\right)$ & Defocus & $Z(4)$ \\
\hline 2 & 2 & $\sqrt{6} p^{2} \cos (2 \theta)$ & Astigmatism $0^{\circ} / 90^{\circ}$ & $Z(5)$ \\
\hline 3 & -3 & $\sqrt{8} p^{3} \sin (3 \theta)$ & & $Z(6)$ \\
\hline 3 & -1 & $\sqrt{8}\left(3 p^{3}-2 p\right) \sin (\theta)$ & Coma along y-axis & $Z(7)$ \\
\hline 3 & 1 & $\sqrt{8}\left(3 p^{3}-2 p\right) \cos (\theta)$ & Coma along $\mathrm{x}$-axis & $z(8)$ \\
\hline 3 & 3 & $\sqrt{8} p^{3} \cos (3 \theta)$ & & $Z(9)$ \\
\hline 4 & -4 & $\sqrt{10} p^{4} \sin (4 \theta)$ & & $Z(10)$ \\
\hline 4 & -2 & $\sqrt{10}\left(4 p^{4}-3 p^{2}\right) \sin (2 \theta)$ & Secondary Astigmatism & $Z(11)$ \\
\hline 4 & 0 & $\sqrt{5}\left(6 p^{4}-6 p^{2}+1\right)$ & First order Spherical & $Z(12)$ \\
\hline 4 & 2 & $\sqrt{10}\left(4 p^{4}-3 p^{2}\right) \cos (2 \theta)$ & Secondary Astigmatism & Z(13) \\
\hline 4 & 4 & $\sqrt{10} p^{4} \cos (4 \theta)$ & & $Z(14)$ \\
\hline$:$ & $:$ & : & : & \\
\hline 6 & 0 & $\sqrt{7}\left(20 p^{6}-30 p^{4}+12 p^{2}-1\right)$ & Second order Spherical & $Z(24)$ \\
\hline 8 & 0 & $\sqrt{9}\left(70 p^{8}-140 p^{6}+90 p^{4}-20 p^{2}+1\right.$ & Third order Spherical & Z(39) \\
\hline
\end{tabular}

Table 2: Mathematical representation and meaning of various Zernike modes where $\theta$ and $p$ are the radial and angular coordinates. The lower order Zernike modes represent a range of known aberrations and the higher orders more complex aberrations. Spherical aberrations are of particular interest as these represent the Zernike circle polynomials of higher even $\mathrm{n}$ order and zero kind: aberrations with no azimuthal variation. 


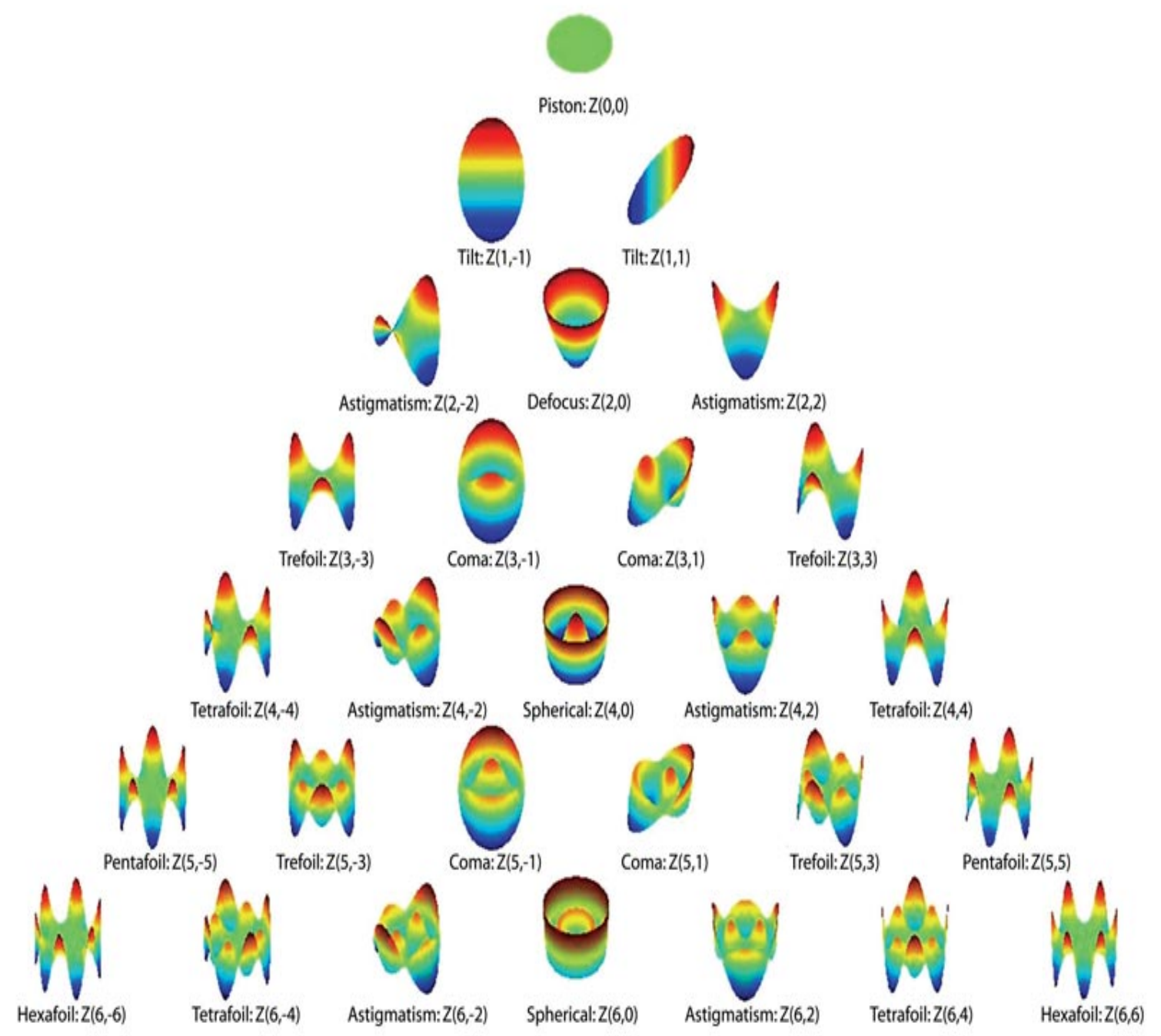

Figure 6: Schematic representation of the various Zernike modes $Z(n, m)$, known as the Zernike pyramid (Costa and Ferrer-Blasco, 2010). Subscript $n$ indicates radial order, which gives the row number in the pyramid. Superscript $m$ indicates azimuthal frequency, which gives the column number in the pyramid.

Zernike polynomials are generally represented with a pyramid structure, commonly referred to as the Zernike pyramid (Figure 6). This simple structure serves to illustrate the increase in complexity for the various orders. For each order the number of polynomials is one more than the previous order. It also serves to illustrate the close relation of the higher and lower orders. Zernike polynomials with the same azimuthal frequency, $m$, maintain the fundamental relation with the primary aberration regardless of the order, $n$. 


\subsection{DISCRETE REPRESENTATION}

Discrete reconstruction serves as an alternative to analytical reconstruction. The discrete reconstruction was developed as the first wavefront representation method. It rapidly lost popularity due to lack of intuitive visualization and manipulation. Recently, the approach has been re-adopted by vision science, which favours the method for being able to represent higher frequencies at the cost of discontinuities. The discrete representation produces lower RMS, in comparison to the analytical representation, as regions with high variations will not be smoothed out.

Instead of decomposing the wavefront, in terms of polynomials, the surface of the wavefront is considered piecemeal giving a measure of flatness within zones instead of over the whole pupil area. These regions generally are represented as square or rectangular elements. The zones are chosen to describe the local gradient measurement, which coincides with the region of the points (the method for reconstruction is described in further detail in the zonal reconstruction section 7.7). Therefore the wavefront, $w$, surface is the result of a point-by-point self-consistent solution to the wavefront under the vector gradient equation (Panagopoulou and Neal, 2004; Tyson, 2010).

\subsection{WAVEFRONT RECONSTRUCTION METHODS}

In order to reconstruct the wavefront from experimentally determined parameters, various techniques have been developed. These are split into two main categories: wavefront sensors and direct sensing. Wavefront sensors, which are the most popular approach, incorporate a microlens array that segments the beam onto a detector. Each beamlet so generated, probes a selected area, from which the wavefront is calculated.

\subsubsection{WAVEFRONT SENSORS}


Acquisition and analysis of the wavefront can be carried out by various wavefront sensors (WFS). There are many methods available (pyramid wavefront sensor, curvature wavefront sensor etc.): However the predominant types are the ShackHartmann WFS and the Tscherning WFS described below. Approaches vary depending on application: the Shack-Hartmann WFS is the most common approach in astronomy (Tyson, 2010) while the Tscherning approach is used commonly in clinical practice (Kaemmerer et al., 2000).

\subsubsection{SHACK-HARTMANN WAVEFRONT SENSOR}

The Shack-Hartmann (Hartmann, 1900; Platt, 2001; Tyson, 2010) WFS was developed to correct for atmospheric aberrations in an effort to monitor satellites from ground based telescopes (Schwiegerling and Neal, 2005). Nowadays the Shack-Hartmann approach has become the most common WFS. The design, represented in Figure 7, is made up of two essential components: a lenslet array and a detector. Light incident on the grid of lenses is focused on the detector creating a series of focal spots. Planar light creates a regular grid of spots at given intervals. The fundamental principle is that the position of these focal points is directly related to the average gradient of the wavefront across the lenslet. A distorted wavefront results in focal spots misplaced or missing, and intensity loss. There are three basic steps to the analysis process: determination of the spot positions, conversion to wavefront gradients and wavefront reconstruction. In modern optical arrangements Shack-Hartmann WFS are used in real-time to monitor wavefront distortions and provide information so that an active optical correction unit can perform corrections (Neal et al., 2002). 

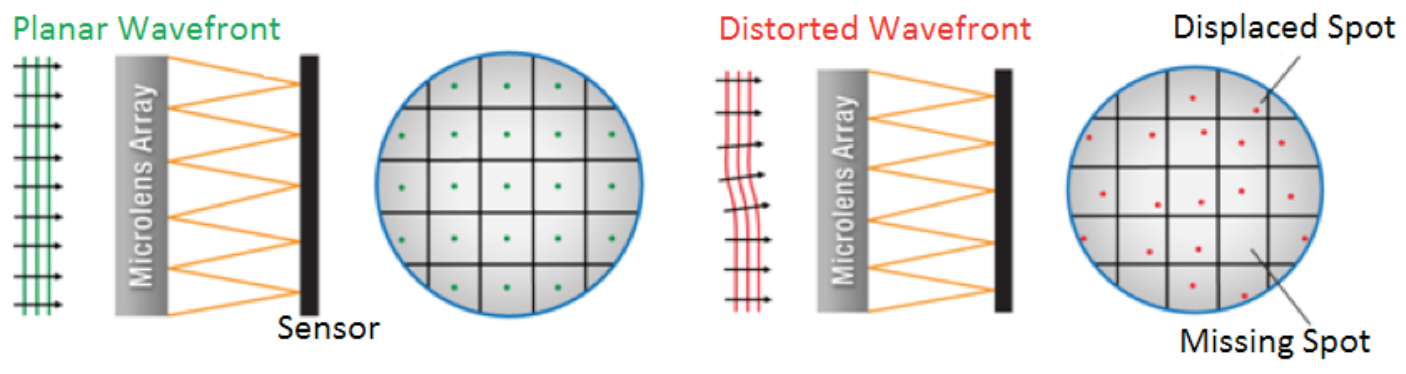

Figure 7: Shack-Hartmann WFS; on the left a uniform wave without distortions results in a uniform and equally spaced grid of spots on the sensor, on the right a distorted wavefront is incident creating a variation of the spots. Image adapted from Thorlabs.com.

The closed-loop wavefront correction approach, described above, requires a reference source to act as a basis of comparison between wavefronts. In astronomy a distant guide star, produces a flat and perpendicular wave to the optical axis, which acts as a reference (Tyson, 2010). However, in microscopy, wavefront sensing is not as straightforward. The three-dimensional nature of the sample means that the reference source would result in multiple wavefronts due to sample heterogeneity. To deal with this issue a reference may be taken with a flat wave, using a highly reflective surface such as a mirror in the object plane - thus correcting for system aberrations (Tyson, 2010).

The main disadvantages of simultaneous wavefront acquisition (Shack-Hartmann, Tscherning) are sufficient signal and the overlapping/crossover effect. In highly aberrated samples, inconsistencies occur in regions where the local wavefront slope is exceedingly steep, which allocates the focal spot in a neighbouring lenslet's "area". This limitation makes it impossible to correlate the spot with the appropriate lenslet. It is possible, however, to minimize the potential of these results by optimizing the system. The overlapping and crossover effects may vary with the size of the individual lenslets and the amplitude of the wavefront being measured. It is necessary to find an ideal aperture size balance, as a larger aperture will provide more light and smaller focal spots while a smaller aperture provides increased sampling resolution (Cervino et al., 2007; Rozema et al., 2005). 


\subsubsection{TSCHERNING WFS}

In essence, the Tscherning (Mrochen et al., 2000) WFS approach follows the same principles and practical implications as the Shack-Hartmann method. It requires a reference source to act as a basis of comparison for the focal spots generated, follows the three basic steps in terms of analysis and suffers from overlapping and crossover. The advantage of the Tscherning aberrometer is that it uses not one, but a group of beams incident on the sample to sense the wavefront. In the Tscherning approach, beamlets are produced through a Tscherning screen or a microlens array which creates beamlets of light that illuminate the sample (Harmening et al., 2007; Kaemmerer et al., 2000). Figure 8 is a schematic of the TWS used in clinical research.

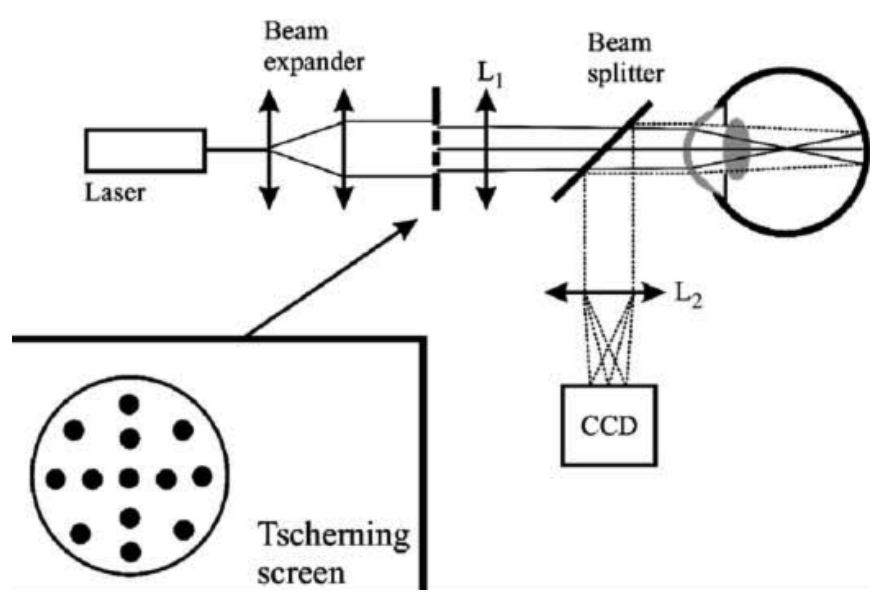

Figure 8: Schematic of a TWS used to evaluate distortions reflected from the retina.

Laser light incident on the Tscherning screen produces beamlets, which are projected on the retina. The wavefront is calculated from the displacements presented on the CCD camera. Lenses denominated as $L$. Image reproduced from (Rozema et al., 2005).

In the Tscherning configuration, a mirror or a model sample (i.e. eye with 20/20 vision) serves to provide a reference environment free from sample aberrations. The positions of the reference spots are configured to act as origins for the spot displacement calculations. The key difference between Shack-Hartmann and Tscherning wavefront sensors is that the Tscherning is a discrete re-imaging system. 


\subsection{WAVEFRONT RECONSTRUCTION AND ANALYSIS}

Three essential steps are necessary to acquire a phase map that corresponds to the distortion detected in the focal plane. The information flow chart below illustrates the steps necessary in wavefront analysis.

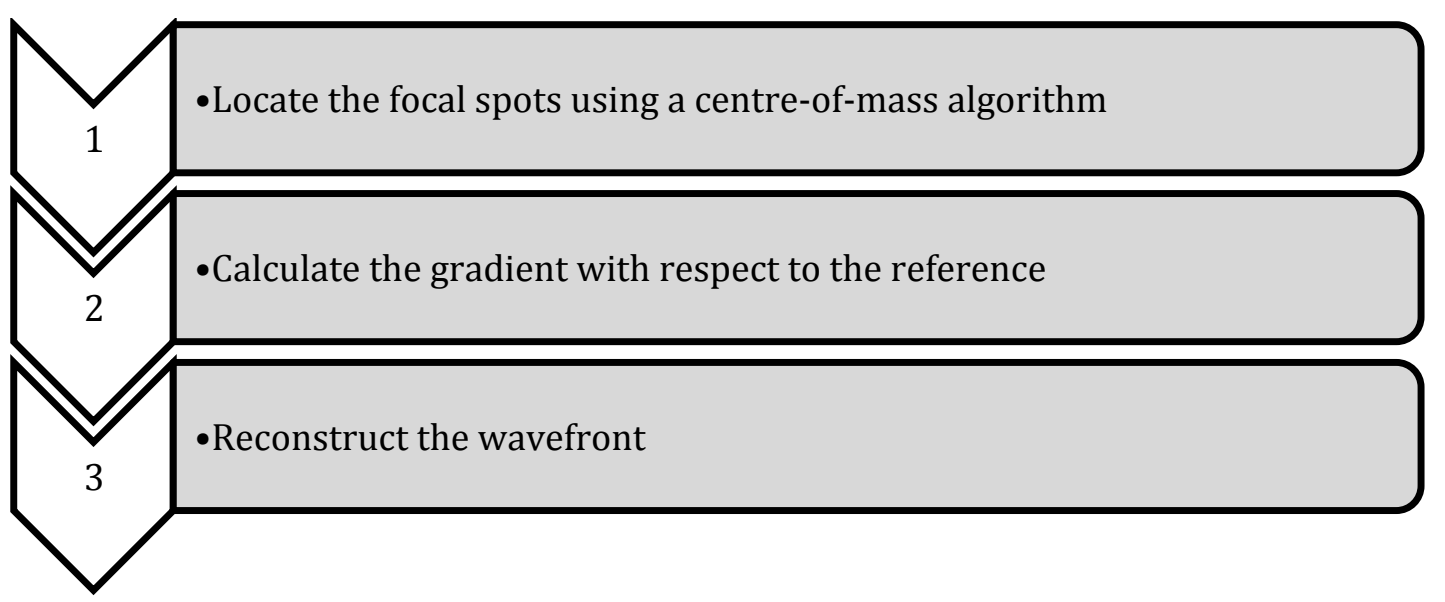

The first step is to locate the focal spots. This approach generally makes use of the centre-of-mass of each focal spots. Each spot, or centroid, has an $x$ and $y$ coordinate given by the centre-of-mass expression (Eq.26):

$$
p_{l}^{x}=\frac{\sum_{b=w_{l}^{x}}^{w_{l+1}^{x}} \sum_{b=w_{l}^{x}}^{w_{l+1}^{y}} I_{u v} x_{b}}{\sum_{b=w_{l}^{x}}^{w_{l+1}^{x}} \Sigma_{b=w_{l}^{x}}^{w_{l+1}^{y}} I_{u v}} \text { and } \quad p_{l}^{y}=\frac{\sum_{b=w_{l}^{x}}^{w_{l+1}^{x}} \Sigma_{b=w_{l}^{x}}^{w_{l+1}^{y} I_{u v} y_{b}}}{\sum_{b=w_{l}^{x}}^{w_{l+1}^{x}} \sum_{b=w_{l}^{x}}^{w_{l+1}^{y} I_{u v}}}
$$

Where $b$ is the pixel brightness, $x$ and $y$ are pixel coordinates, and $w$ represents the boundaries for a given ROI.

The gradient for each lenslet is determined as the difference between the measurement centroid locations and those of a reference centroid taken with a flat wavefront, over the focal length (Eq. 27)(Dai, 2008).

$$
\frac{d \varphi}{d x}=\frac{p_{l}^{x}-p_{\text {lref }}^{x}}{f} \quad \text { and } \quad \frac{d \varphi}{d x}=\frac{p_{l}^{y}-p_{\text {lref }}^{y}}{f}
$$

Where $f$ is the focal length of the lenslet array and $p$ represents the centre-of-mass coordinates for both measurement and reference of a given lenslet. 
The final step in analysing aberrations is to produce a wavefront. The wavefront, $w$, is related to the corresponding $x$ and $y$ components at any giving point by Eq. 28 .

$$
\Delta w=\frac{\delta w}{\delta x} u+\frac{\delta w}{\delta y} v \quad 28
$$

Where $u$ and $v$ are the unit vectors.

There are two main approaches towards wavefront reconstruction: Modal (Section 7.6) and Zonal reconstruction (Section 7.7). The zonal reconstruction method provides a uniquely detailed representation of the wavefront at various areas resulting in representations with higher spatial frequencies. However, the zonal approach does not provide for a direct interpretation in familiar optical terms (defocus, spherical aberrations etc.) or for an intuitive visualization. It was also demonstrated that for lower order aberrations, Zernike polynomials produce lower RMS (Southwell, 1980).

\subsection{WAVEFRONT CALCULATION: MODAL RECONSTRUCTION USING ZERNIKE POLYNOMIALS}

Zernike polynomials represent a combination of 3-dimensional data to describe aberrations of a wavefront. In order to represent a wavefront with unknown optical properties, the modal reconstruction uses wavefront slope measurements to fit the coefficients of a set of polynomials. Once the adequate coefficients are retrieved the aberration wavefront phase map can be calculated.

For practical purposes, Zernike polynomials need to be truncated, producing a wavefront with $N_{A}$ coefficients proportional to the polynomials (Eq. 29). Establishing the necessary order of polynomials to fit is generally sensitive to the degree of aberration present. Ignoring computational costs and complexity, fitting to a very high number of orders (i.e. $10^{\text {th }}$ order) would consistently show minimum RMS. However, limitations in the wavefront sensor design also restrict the quantity of polynomials, which can be calculated. The number of beamlets produced by the lenslet architecture has a linear relation to the number of Zernike polynomials 
which can be fit reliably (Porter et al., 2006). Nevertheless, it was shown for microscopy that in the majority of cases, diffraction limited results can be achieved using Zernike modes up to the $3^{\text {rd }}$ order (Booth et al., 1998b).

Modal reconstruction of a wavefront uses slope measurements to fit coefficients of a set of functions. From the wavefront equation using Zernike polynomials:

$$
w(p, \theta)=\sum_{i=1}^{N_{A}} A_{n}^{m} Z_{n}^{m}(p, \theta) \quad 29
$$

The sum above disregards piston since it does not contribute to a change in the wavefront or point-spread function of the microscope. As Zernike polynomials are continuous, the first derivative provides a wavefront gradient in $x$ and $y$ which can be compared to the measured gradient values. The appropriate set of coefficients is obtained by least square fitting (Carvalho, 2005; Liang and Williams, 1997).

Taking the partial derivatives with respect to $x$ and $y$ for the total number of sampling points, $k$ :

$$
\left.\frac{\delta w}{\delta x}\right|_{l}=\left.\sum_{i=1}^{N_{A}} \frac{\delta A_{n}^{m} z_{n}^{m}(p, \theta)}{\delta x}\right|_{l},\left.\frac{\delta w}{\delta y}\right|_{l}=\left.\sum_{i=1}^{N_{A}} \frac{\delta A_{n}^{m} z_{n}^{m}(p, \theta)}{\delta y}\right|_{l}
$$

For all points 1:l:k. These equations (Eq. 30) result in a matrix:

$$
s=E_{M} A
$$

$\mathrm{E}_{\mathrm{m}}$, represents a matrix containing the partial derivatives of $Z$ with respect to $x$ and $y$. Using a single value decomposition (SVD) of the matrix $E$, it is possible to solve the equation above with respect to $A$.

\subsection{WAVEFRONT CALCULATION: ZONAL RECONSTRUCTION}

Similar to modal reconstruction, zonal reconstruction makes use of the gradients determined in sensing to build a description of the wavefront. The reconstruction is performed over a limited zone, instead of analytically over the whole surface. The zone is chosen to match the area described by each gradient measurement, generally a regular square grid. 
There are three different approaches to zonal reconstruction: Fried, Hugdin and Southwell illustrated in Figure 9.
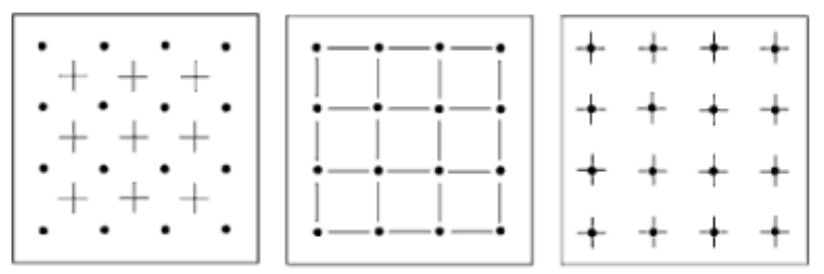

Figure 9: Zonal reconstruction configurations based on the $x$ and $y$ slope components of the gradient. First square represents the Fried approach, the second the Hugdin and the far right is the Southwell configuration. The horizontal and vertical lines are the $x$ and $y$ component, respectively (Dai, 2008).

The Fried method (Figure 9, left) is suitable for shearing interferometers and ShackHartmann aberrometers, the Hugdin approach (Figure 9 middle) $x$ and $y$ do not coincide (Zou and Rolland, 2006). The Southwell (Figure 9, right) is more suitable for Tscherning and the Shack-Hartmann.

The Southwell configuration (Southwell, 1980) determines that in a $N x N$ grid, the average slope is given by the difference between two adjacent phase values separated by $d_{s}$ (Eq. 32, Eq. 33):

$$
\begin{aligned}
& \frac{S_{u, v+1}^{x}+S_{u, v}^{x}}{2}=\frac{w_{u, v+1}+w_{u, v}}{d_{s}},(u=1, N ; v=1, N-1) \\
& \frac{s_{u+1, v}^{y}+s_{u, v}^{y}}{2}=\frac{w_{u+1, v}-w_{u+1, v}}{d_{s}},(u=1, \mathrm{~N} ; v=1, \mathrm{~N})
\end{aligned}
$$

Equation 32 and Equation 33 above can be represented in a single matrix (Eq. 34) as:

$$
C s=E_{S} w
$$

Where $s$ is a column vector of the slope measurements, $w$ is a column vector of the unknown wavefront and $C$ and $\mathrm{E}_{\mathrm{S}}$ are sparse rectangular matrices. The wavefront then corresponds to the graphical representation of the $w$ matrix (Dai, 2008). 
For the case of $E_{S}$ being a square matrix with full rank the solution simply corresponds to a matrix inverse:

$$
w=E_{S}{ }^{-1} C s \quad 35
$$

In which $E_{S}^{-1}$ is the inverse of $E_{S}$. For all cases below, the matrix $E_{S}$ has full rank. However, in the general case $E_{S}$ is rectangular as the number of measured points is larger than the phase values to be estimated. In that case, a SVD is performed.

\subsection{ZONAL AND MODAL FITTING}

Recent approaches have been developed which make use of a combination of the two reconstruction methods. Regionalized scanning of the sample provides diverse local phase calculations, which are then fit to Zernike polynomials to appropriately characterize the amplitude of the various distortions. A polynomial fit is performed locally over a masked region of the surface. The mask is suitably displaced over the entire surface providing a zonal approach with a modal fit. Unlike a microlens array, the masked region does not necessarily need to correspond to any specific region. Fitting over many zones diminishes the affect of smoothing while providing the analytical advantage of Zernike modes (Espinosa et al., 2010). However, an alternative approach demonstrates how reconstruction based on the use of Zernike polynomials does not require a mask (Bowman et al., 2010). The optical configuration is similar to a Tscherning wavefront sensor, but beamlets are produced using a SLM. A blazed grating is produced in various regions and selectively images portions of the sample. A blaze grating function consists of saw tooth function with constant spatial frequency with the purpose to deflect incident light at a certain angle with the highest possible optical efficiency (Goodman, 1996). The approach developed demonstrates flexible regionalized aberration insight. Alternating the positions of the beamlets generated using the SLM would provide overlapping reconstruction zones. The information from overlapping zones could be weighted equally and the combination would show improved sensitivity with reduced risk of overlapping of the displaced beamlets. 


\subsection{DIRECT SENSING}

In order to obtain an optical solution to the deterioration of signal produced by samples at an increased depth; direct sensing methods were developed as an alternative to wavefront sensing in microscopy. These approaches mainly consist of algorithms, based on iterative approximations, which maximize a specific image parameter such as brightness, position, shape or intensity. Ordinarily, iterative approximations are performed using sequential optimizations of an additive calculation of the coefficients, while monitoring a particular metric function (i.e. intensity). Generally performed $2 N+1$ times, for $\mathrm{N}$ Zernike modes, acquisition time and SNR are some of the limitations for increased number modes (Débarre et al., 2008; Débarre et al., 2009). Direct wavefront sensing methods such as HillClimbing, Random Search (Wright et al., 2005) and pupil-segmentation (Ji et al., 2010)

Hill-climbing algorithm developed for deformable membrane mirrors (DMM) sequentially selects each actuator of the DMM individually and iteratively steps the voltages from 0-200 V. At every voltage step the PMT value is recorded. The voltage that registers the highest signal on the PMT is saved and the algorithm proceeds to the next actuator. Once the algorithm has registered the highest PMT signal for all actuators, the corrective pattern is projected (Wright et al., 2005). The Random search algorithm starts by randomly selecting an actuator on the DMM that is perturbed with an indiscriminate voltage (Section 10.5). If the PMT detects a signal improvement the actuator voltage is accepted, otherwise rejected. The algorithm consists of iteratively probing random voltages over a user-selected amount of cycles or a programmable stopping parameter (Wright et al., 2005). The pupil-segmentation approach (Section 17.1) consists of illuminating square regions of the pupil in turn. Each square pupil section probed results in a position change in the image plane with respect to the fully illuminated pupil. The variation in position is related to the amplitude of the aberration sensed in each particular sub-region. A zonal wavefront reconstruction is then performed corresponding to the respective phase variations in the pupil of the objective (Ji et al., 2010; Ji et al., 2012; Milkie et al., 2011; Wang and Ji, 2012). 


\section{CHAPTER 2 - MULTIFOCAL MULTIPHOTON FLUORESCENCE LIFETIME MICROSCOPY}

\section{OVERVIEW}

This Chapter describes the experimental development of a multiphoton fluorescence lifetime imaging microscope for high-speed applications in cell biology.

In the context of MPM the development of FLIM has been broadly in two directions chiefly due to the predominantly scanned nature of the excitation. Initially, effort focused on translating methods previously used in confocal FLIM such as frequency domain heterodyne detection (Lakowicz, 1990) to TPM which was aided by the 70 $80 \mathrm{MHz}$ repetition rate of commonly used Ti:Sapphire lasers (Jakobs et al., 2000). With the availability of high-speed TCSPC detection electronics from companies such as Picoquant and Becker \& Hickl in the late 1990's, frequency domain and also at this time a gated lifetime technique spun out of the Gerritsen group (Buurman et al., 1992; Gerritsen et al., 2002) was quickly superseded. TCSPC became the method of choice for laser scanning microscopy based FLIM. For a single beam TCSPC scanning system with a count rate limited typically by the sample, rather than detection electronics, high SNR can only be achieved in conjunction with a relatively long scanning time (typically between 3-5 minutes). A main disadvantage of these systems is then seen as the relatively poor frame rate for acquisitions. Of course one can always sacrifice imaging resolution for speed, which is often the case in FLIM imaging since, whilst often not explicitly stated, binning of pixel data in a manner equivalent to median filtering is endemic in the field. However, use of data processing techniques such as global analysis can allow higher data rates potentially without sacrificing imaging resolution (Barber et al., 2009). Widefield frequency and gated time-domain remain the methods of choice for high-speed FLIM (Suhling et al., 2005). Here too though, resolution is an issue such that Nipkow disk imagers are essential to achieve (near) diffraction limited performance (Straub and Hell, 1998). For MPM specifically, this has led to the development of multifocal FLIM scanning 
systems to exploit the total detection count rate provided by modern TCSPC electronics using a segmented detector (Kumar et al., 2007b).

More generally, multifocal generation in the framework of TPM has been successfully developed using optical beams generated with lenslet arrays (Bahlmann et al., 2007; Buist et al., 1998; Kim et al., 2007), Nipkow-Type microlens array (Bewersdorf et al., 1998), high efficiency beam splitter (Nielsen et al., 2001; Rinnenthal et al., 2013), diffractive optical element (DOE) (Watson et al., 2009) and high-performance electrically addressable SLM (Grier, 2003; Howard et al., 2013; Tyndall et al., 2011).

The practical implementation of an array of microlenses for TPM consists of an expanded and collimated laser beam of a mode-locked Ti:Sapphire laser through which, the illuminating beam is split into small beams, referred to as beamlets, and focused into an array with equally spaced foci, assuming an aberration free environment. A multifocal multiphoton microscope (MMM) scans an object with an array of high-aperture foci, reducing higher order photodamage effects through parallelization, while maintaining the many advantages of single-beam TPM, such as superior live-cell compatibility, reduced photobleaching of non-target planes and optical sectioning. Beam parallelization increases the potential photon count rate proportional to the number of beamlets employed. Fluorescence corresponding to each beamlet is projected on the detection system: CCD, PMT array (Kim et al., 2007) or SPAD simultaneously, permitting high accuracy measurements in reduced time frames (Poland et al., 2013; Poland et al., 2014).

A multifocal illumination system coupled with a multi-channel TCSPC detection architecture lifts the fundamental limitation imposed by count rates in standard single-channel experiments (i.e. photon count rate to avoid pile-up). In Section $10 \&$ 11 of this chapter the development of a multifocal multiphoton FLIM system (MMFLIM/MegaFLI) is described, with the aim of exploiting the parallelisation of FLIM to increase acquisition rate. Parallel detection of single photon counting was achieved using a high number of channels provided by a time-resolved complementary metal-oxide-semiconductor (CMOS) single-photon avalanche diode (SPAD) array 
developed by our collaborators (Henderson Group, University of Edinburgh) (Betta et al., 2011; Richardson et al., 2009a). Section 9 describes the detector specifications.

A number of multifocal generation approaches were investigated to maximize the potential for imaging. The rationale for adapting an SLM based beam array (whilst not immediately logical given the degree of loss associated with the technique) is clearly discussed in section 10 .

Section 11 describes the MMM-FLIM setup, which combines a holographic multifocal excitation with CMOS-SPAD detector in the descanned configuration. A description of the two developmental stages of the system: the non-descanned (Section 12.1) and descanned configuration (Section 12.2) is given in Section 12. Manual and automatic position optimization and alignment procedures are also discussed followed by analysis and implementation of the weighted GerchbergSaxton algorithm to reduce heterogeneities in the detection. Section 13 discusses data analysis, image stitching and alignment.

In Section 14, the imaging performance of the system is characterised with standard samples and techniques along with biological cell imaging of fluorescent proteins in live cells and 3D optical stacks in vitro and in vivo with diffraction limited resolution. The data is discussed in context of single beam scanning FLIM and includes future prospects for the project, presented in Section 15 and Section 16, respectively. The principle output of this chapter is an optically engineered, fully functioning, multifocal TPM system with a 2D SPAD array detector that offers practical and innovative advantages for FLIM. 


\section{TIME RESOLVED DETECTION SYSTEM FOR PARALLELISED TCSPC}

In order to parallelise confocal or multiphoton FLIM excitation and emission detection in FLIM, one requires a detector assembly capable of detection of singlephotons, deriving their arrival time relative to an excitation source and accepting the multiplied counting rate. Through collaboration with colleagues at the University of Edinburgh (Robert Henderson and co-workers) access has been given to a number of SPAD in an array configuration. In this thesis we discuss only the Megaframe 32 device (a SPAD array consisting 32×32 SPADS with associated timedomain counters) (Richardson et al., 2009a). In this section, the broader field of SPAD technology is discussed.

A SPAD is, in effect, a p-n junction biased above breakdown operating in Geigermode in which a digital pulse is produced for each photon incident on the active area (Cova et al., 1996). Previous reports show that a SPAD detection architecture can be used in confocal microscopy (Böhmer et al., 2001), fluorescence correlation spectroscopy (FCS) (Maiti et al., 1997; Ray et al., 2010), microarrays (Marangoni et al., 2010), flow cytometry (Li et al., 2012b), 3D acquisition (Aull et al., 2002; Niclass et al., 2008; Niclass et al., 2005), FLIM (Gersbach et al., 2010), single-molecule FRET (Ingargiola et al., 2013; Michalet et al., 2013; Panzeri et al., 2013), astrophysics (Zappa et al., 2007), astronomy (Nightingale, 1990), quantum mechanics (Rarity and Tapster, 1990), quantum cryptography (Chiangga et al., 1999), diode laser characterization (Cova et al., 1989) and optical fibre testing (Ripamonti et al., 1990).

Detectors with a multiplication of charge carriers sufficiently high such as PMTs and APDs are capable of single photon detection. PMTs exhibit high quantum efficiency and low noise, but are bulky and unsuitable for large arrays (i.e. 1024) (Hakamata, 2006; Kim et al., 2007). APDs based on solid state devices, have a small size, low bias voltage and reduced power consumption (Zappa et al., 1996). This makes semiconductor APDs suitable for compact two-dimensional imaging systems. There exist two operation methodologies for APDs: linear mode (Huntington et al., 2007), which typically cannot provide adequate gain with satisfactory noise to make the photocurrent from a single photon detectable; and Geiger mode which relies on 
active quenching after an avalanche is ignited by an incident photon. Operating in Geiger mode provides single photon sensitivity, and therefore these devices have been termed single-photon avalanche diodes (Aull et al., 2002).

At the bias, the high electric field (above $10^{5} \mathrm{Vcm}^{-1}$ ) is sensitive to an incident single charge carrier (Betta et al., 2011). Therefore a perturbation in the depletion layer triggers a self-sustaining avalanche. The avalanche, started by the initial impact ionization of a single photon, is a consequence of electrons and holes multiplying faster than they can be extracted, leading to an exponential growth of current in a time scale of tens of picoseconds. The quenching circuitry quenches the SPAD and it remains quenched until the next reset in which it is driven above breakdown voltage, therefore 'resetting' the device back into operation. The time needed for quenching/recharging introduces a dead time; a time frame for which a photon is not detected. A SPAD acts as a digital device, switching from an off state to a conducting state in response to the detection of an avalanche (Cova et al., 1996; Villa et al., 2012).

The photon detection efficiency of a SPAD varies linearly with the probability of photon absorption, (quantum efficiency), the probability of carrier collection, (injection to multiplication region) and the probability that collected carrier initiates detectable avalanche (Villa et al., 2012).

The SPAD has temperature dependency, as carriers thermally generated and emitted from the trap levels can also trigger the avalanche. This effect is termed the dark-count rate (DCR). Although operating temperature is the main contributor to the DCR, any non-photo generated carrier such as diffusion from neutral regions, band-to-band tunnelling or after-pulsing adds to the DCR. After-pulses result from carriers trapped during an avalanche current flow and then released by trapping centres, which affect the linearity and generate additional noise (Gulinatti et al., 2011).

The ideal SPAD array would have all in-pixel architecture, maximum fill factor, high quantum efficiency and time resolution, while providing minimum dark-count, dead time and optical crosstalk. At the present state, SPAD development selectively 
addresses a few of these objectives, tailoring the detector for a specific application. For practical purposes, compromises need to be made to optimize on specific parameters; i.e. to reduce optical crosstalk it becomes necessary to introduce a guard ring which sacrifices fill factor. With current technology it is more sensible to choose the SPAD with the application in mind (Marangoni et al., 2010).

Single photon sensitivity in CMOS SPADs may be obtained via time-gating (Tosi et al., 2009), a TAC (Swann et al., 2004) or a TDC (Richardson et al., 2009a). An individual CMOS-SPAD equipped with a TDC is capable of TCSPC (Niclass et al., 2007). The TDC measures the time interval between the SPAD recharge signal and the moment a first Geiger event occurs. Converters can be based on a minimum gate delay (Arai and Ikeno, 1996) or a ring oscillator (De Heyn et al., 2007). Table 3 shows a comparison of currently available TDC ring oscillator based time-resolved SPAD arrays, adapted from (Villa et al., 2012). 
Table 3: Comparison of various SPADs (Villa et al., 2012).

\begin{tabular}{|c|c|c|c|c|c|c|}
\hline Work & $\begin{array}{c}\text { Technology } \\
\text { (nm) }\end{array}$ & Architecture & $\begin{array}{c}\text { DCR at } \\
20^{\circ} \mathrm{C} \\
\left(\mathrm{Hz} \mu^{-2}\right)\end{array}$ & $\begin{array}{c}\text { Fill } \\
\text { factor } \\
(\%)\end{array}$ & $\begin{array}{c}\text { TDC } \\
\text { range } \\
\text { (ns) }\end{array}$ & $\begin{array}{c}\text { Precision } \\
\text { (ps) }\end{array}$ \\
\hline $\begin{array}{c}\text { (Villa et al., } \\
\text { 2012) }\end{array}$ & 350 & All in-pixel & 0.021 & 3.14 & 320 & 175.1 \\
\hline $\begin{array}{l}\text { (Markovic } \\
\text { et al., 2013) }\end{array}$ & 350 & All in-pixel & 12.7 & 0.5 & 160 & 17.2 \\
\hline $\begin{array}{l}\text { (Gersbach } \\
\text { et al., 2009) }\end{array}$ & 130 & All in-pixel & 189 & 2.3 & 120 & 111 \\
\hline $\begin{array}{l}\text { (Richardson } \\
\text { et al., } \\
\text { 2009a) }\end{array}$ & 130 & All in-pixel & 0.5 & 1 & 55 & 52 \\
\hline $\begin{array}{l}\text { (Niclass et } \\
\text { al., 2008) }\end{array}$ & 350 & Multiplexed & 16.8 & 6 & 100 & 106.2 \\
\hline
\end{tabular}




\subsection{MEGAFRAME}

The detection system in the Megaframe32, (Figure 10) consists of all in-pixel architecture with high sensitivity equipped with a 10-bit TDC capable of operating in a TCSPC mode, an exclusive technology relevant to this thesis acquired via a collaboration with the Henderson group (Richardson et al., 2009a). The Megaframe 32 is a $32 \times 32$ CMOS SPAD array with a total dimension of $1.6 \times 1.6 \mathrm{~mm}$. Each pixel has a $55 p$ s time resolution, 50 ns dead time, exhibits a quantum efficiency of $28 \%$ at $500 \mathrm{~nm}$ while displaying an average dark count of $50 \mathrm{~Hz}$ at room temperature and can operate in time-correlated mode for lifetime imaging or in time-uncorrelated mode for intensity imaging (Li et al., 2009). Synchronized using a laser trigger, the on-pixel TDCs generate raw arrival time data, which is sent to a PC and postprocessed by curve fitting software. To increase transfer speed to the host PC, the raw arrival time data can be processed on a field programmable gate array (FPGA) using a centre of mass method (CMM), achieving video-rate FLIM imaging for biological applications (Li et al., 2011). However, the small sizes of the SPAD active area (6 $\mu \mathrm{m}$ diameter), coupled with a low fill factor ( 1\%) are a significant disadvantage for collection efficiency in widefield imaging mode. Optically conjugating the photosensitive area of the SPAD pixel with fluorescence generated from the excitation serves to optical amplify the fill factor (Krstajić et al., 2013). It was the utility of the detector for parallelising detection coupled with this optically amplification of fill-factor that led to the development of the MegaFLI technique. 

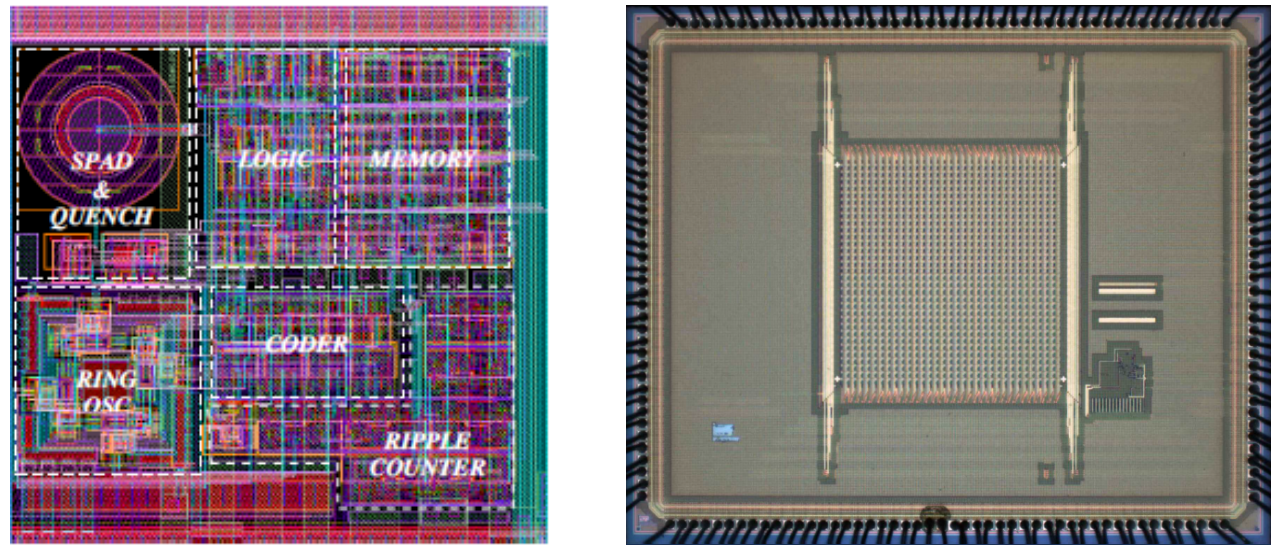

Figure 10: Single Photon Avalanche Diode; representation of individual SPAD and circuits (left) and entire 32×32 SPAD array (right)

Structurally, the SPAD integrated on a $130 \mathrm{~nm}$ imaging technology uses a TDC ring oscilator which is embedded with buffer memory and glue logic (Richardson et al., 2009a). A custom motherboard provides the interface between the FPGA circuit board (XEM3050, Opal Kelly, Oregon, USA) and the Megaframe camera.

The device can operate in various modes, but throughout this chapter only intensity (photon counting) and TCSPC mode were utilised. Intensity mode configures the SPAD detection to count ignitions over a user specificed time frame. This mode serves to measure the intensity of a constant or slowly varying optical signal, facilitating alignment. The second operation method is TCSPC. This approach operates in a reversed mode (Section 1), in which the quenching circuit is sychronized with a pulsed laser source providing the STOP signal (Richardson et al., 2009a).

The Megaframe is equivalent to 1024 parallel TCSPC channels. In the current design each pixel has a TDC capable of production a photon arrival timestamp with 55 ps resolution. The typical timestamp for each SPAD is 10 bits long resulting in 56 ns dynamic range covering the fluorescence decay times for most fluorophores. Recent advances provided by the Megaframe developers have been implemented. The improvement increases per-pixel data transfer rate from $4700 \mathrm{cps}$ to 95000 cps, in a user selected 64-pixel region. This development has improved data transfer to 16 Mcps, a noticeable advancement over the traditional 1 Mcps of 
typical TCSPC. The firmware only reads out timestamps of 64 pixels out of the total 1024. This feature results in a modification of the timestamp. Each timestamp occupies 16 bits; 10 bits corresponding to the time data and 5 bits of addressing of 64 pixels and one spare bit. As each pixel is a completely independent photoncounter, it is to be expected in such a large array, to have a variability of the IRF peak locations (Krstajić et al., 2013).

In order to perform adequate detection readout, a LabVIEW based interface developed by Dr. Krstajic configures the firmware loaded onto an on-chip FPGA and modifies the array settings and processes the camera output during experiments. The relevant photon counts obtained are sent to a second LabVIEW program created for post-processing the multi-channel data, analysis and plotting (Dr. Simon Poland and myself). The software package controls all aspects of the microscope system and the $x-y$ scanner synchronisation signals and pixel trigger clock. Once acquired, TCSPC data is saved into the Becker and HickI SDT file format (readily available LabVIEW library to write SDT files; routinely used in the group) and subsequently analysed using TRI2 lifetime analysis software (developed by Dr. Barber) (Barber et al., 2009). The data is saved into 180 time bins corresponding to a resolution of $55 \mathrm{ps}$. The Becker \& HickI SDT file format requires data to be saved with temporal bin sizes in factors of 2 so we save 256 bins of data and pad the remaining bins with zero values. SDT file format has limited options for temporal resolution since it is usually associated with $\mathrm{B} \& \mathrm{H}$ hardware and is written into the header file information - resolution of 49ps is therefore chosen - which corresponds to 10 ns over 256 bins (standard arrangement for a FLIM system using 10 ns temporal range). Using standard fluorescence lifetime software, the result is an underestimation of the fluorescence lifetime. Adequate recalibration is performed by multiplying the fit values by $\frac{256}{180}$. This problem could be resolved by using the ICS file format (standard in the group due to its flexibility). However, due to issues with writing ICS files in the LabVIEW environment and in the interests of time, we chose to apply the correction in the post-processing stage. 


\subsection{BECKER AND HICKL PMH-100 VS. SPAD}

In order to provide a brief comparison between the photon counting performance of a standard PMT for lifetime imaging with the SPAD array, a B\&H PMH-100 (Becker, 2008) and the SPAD were placed in turn in the descanned detection path (Figure 32). The emission from a single excitation beam (800 $\mathrm{nm}$ excitation) stationary on a green florescent block was focused onto the surface of the PMT or an individual SPAD (the SPAD was chosen displaying low DCR within the array). Measurements were acquired over $100 \mathrm{~ms}$ with a $10 \mathrm{~mW}$ illumination. Figure 11 shows photons counts per ms, averaged over 3 acquisitions.

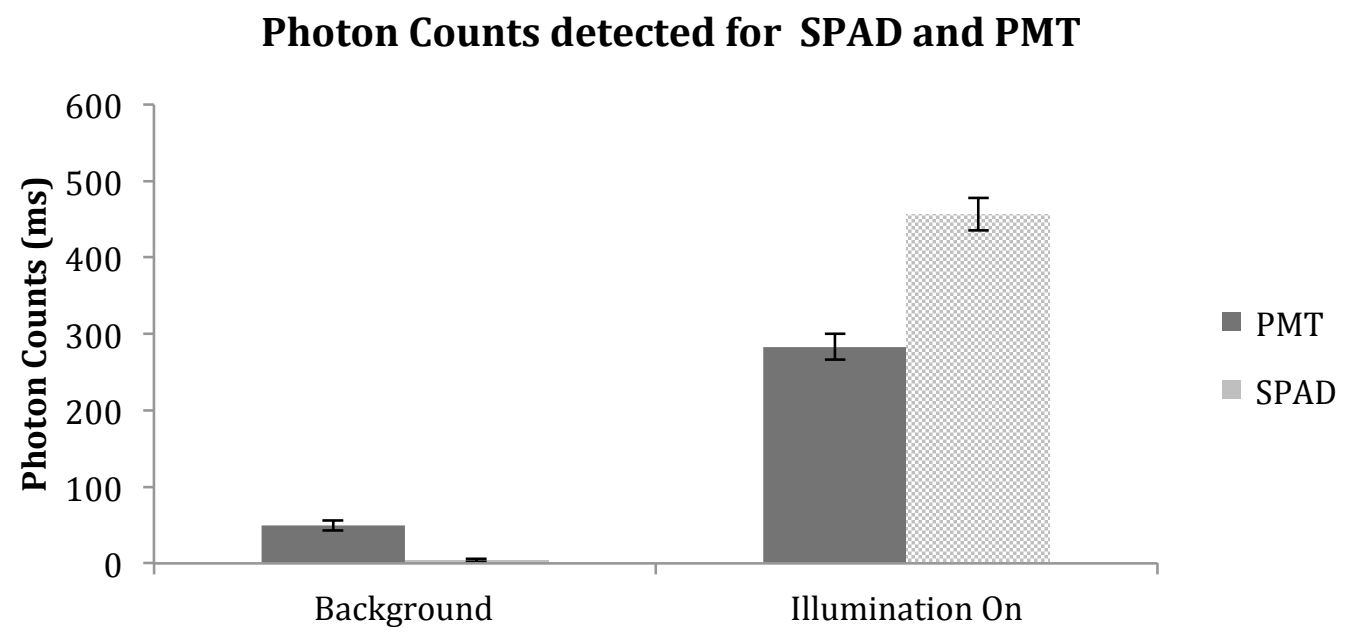

Figure 11: Photon count comparison between a B\&H PMH-100 and a SPAD in the non-descanned detection. Fluorescence generated by a $10 \mathrm{~mW}$ excitation of a fluorescent block is shown as counts per ms. Error bars calculated as $\sqrt{N}$.

The background counts detected using an individual SPAD are significantly lower; this can be attributed to a smaller active area compared to the PMT (Michalet et al., 2008). However, once the fluorescence is illuminating the detectors it is possible to verify the SPAD has an $\times 1.94 \pm 0.5$ improvement in detection efficiency over the $\mathrm{PMH}-100$. The $\mathrm{PMH}-100$ has a $15 \%$ quantum efficiency at $500 \mathrm{~nm}$, which correlates to approximately $28.95 \%$ for the SPAD, within error of published results (Richardson et al., 2009a). 


\section{MULTIFOCAL GENERATION}

In order to optically amplify the fill-factor of the Megaframe camera, the generation of multiple focal volumes was investigated. Fluorescence imaging techniques based on multifocal generation have recently gained significant attention over a range of techniques with the primary objective to improve acquisition rates. Recent developments in multifocal STED using diffraction gratings, show live-cell imaging based on reversible saturable optical fluorescence transitions acquired $<1 \mathrm{~s}$ (Chmyrov et al., 2013). Multifocal structured illumination acquisition based on a lenslet array with acquisition provided by a sCMOS camera has produced faster than video-rate super-resolution of microtubules (York et al., 2013). Advances in the frequency-domain FLIM include frequency-multiplexed in-vivo; in which each pixel space of an SLM encodes a distinct frequency which is recovered by means of signal frequency demodulation (Howard et al., 2013).

The principal method investigated in this thesis was the use of a holographic projection into the focal plane using a SLM as a spatially programmable phase plate, enabling highly flexible illumination. Whilst more light efficient, the inherent rigidity of refractive micro-lenses or diffractive optical element (DOE) typically used to generate excitation beamlets in multifocal microscopy comes at the expense of individual beamlet manipulation (i.e. for alignment or geometry modification). In the case of a SLM, a phase pattern permits considerable manipulation of the excitation plane (Moreno et al., 2008). The phase mask corresponds to the Fourier transform of the desired intensity distribution. The ability of the SLM to represent complex-valued image enables intensive information processing functions using the Fourier transform properties of light. Such devices have been used extensively in structured illumination (Kner et al., 2009), STED (Lenz et al., 2011), holographic optical tweezers (HOT) (Grier, 2003) and adaptive optics (Neil et al., 2000b).

Prior to implementing the holographic projection methodology with an SLM as the beamlet generating method, various alternatives were investigated including a refractive lenslet array and programmable Fresnel lenses (using an SLM). This 
section details the approaches concerned with the generation of multiple beams for multifocal FLIM.

\subsection{REFRACTIVE MICROLENS ARRAY}

A commercially available lenslet array (MLA150-5C - Thorlabs Ltd; 5.2mm focal length and $150 \mu \mathrm{m}$ pitch) was used in a Tscherning wavefront sensor (TWS) configuration constructed to establish the sensitivity of the beamlet pattern to optical aberrations. Data generated by the Tscherning arrangement serve as a test bed for considering the effect of aberrations on propagating beam arrays.

The MLA150-5C microlens array is a square grid type with plano-convex lenses on a fused silica substrate, with a fill factor of $74.5 \%$. The microlenses have chrome apertures surrounding the lenses, which provide reflectivity of approximately $25 \%$, therefore generating significant back reflections but reducing diffraction effects and ghosting in the focal plane. The beamlet array was focused using a Nikon 0.9 NA, 40x ELWD Plan Fluor objective with spherical aberration collar correction collar to enable variable working distance [Nikon Instruments Inc., Japan]. On reflection from a mirror sample in the focal plane, the resultant beams are re-directed via a 50:50 beam splitter cube onto a CMOS camera (Thorlabs UK, Ltd: Part No. DCC1545M). Laser light incident on the lens array are focused on the mirror sample, creating a series of focal spots. Planar light (flat wavefront) creates a regular grid of spots at intervals given by the re-projection magnification between array primary focal plane and the detection plane. The fundamental principle of a Tscherning WFS is that the position of these focal points is directly related to the average slope of the wavefront across the lenslet. A distorted wavefront results in focal spots misplaced (offset) or missing (offset into the adjacent lenslet 'zone') and intensity loss (loss of beamlet fidelity). Figure 12 is a schematic of the TWS used to evaluate the method. 


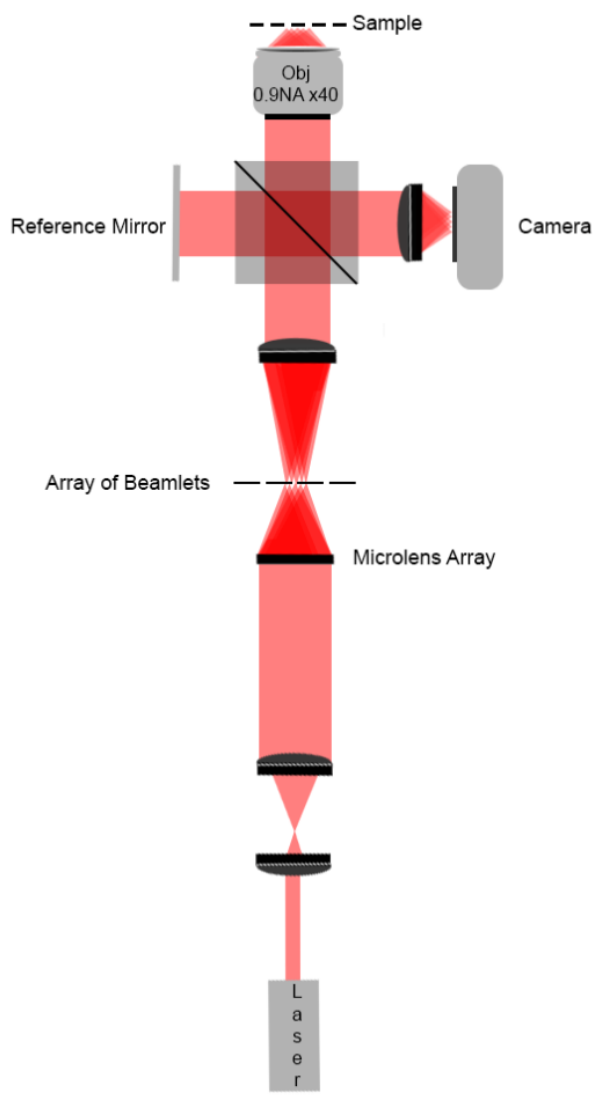

Figure 12: Schematic of TWS setup. The array from the sample plane is directly compared to the beamlets from the reference mirror purposely chosen with a high degree of flatness. The laser was expanded via a telescope (focal lengths $50 \mathrm{~mm}$ and $150 \mathrm{~mm}$ ) and projected onto the microlens array. Recaptured by a $150 \mathrm{~mm}$ lens was focused on the sample using an x40 0.9 NA air objective. Retroreflected aberrated beamlets were separated from the incident beamlets via a beam splitter cube and refocused onto a CMOS camera (Thorlabs) by a $200 \mathrm{~mm}$ focal length lens. In modern set-ups, the TWS is used in real-time to monitor wavefront distortions such that an active optical correction unit can correct aberrations in closed loop. The TWS requires a reference source (reference mirror) to act as a basis of comparison for the focal spots generated. To investigate generation of multiple excitation points using a microlens array and their sensitivity to optical aberrations, a highly reflective mirror was used to re-image the beamlets from the sample to the detection plane (Figure 12). Spherical aberrations were induced (using the correction collar) to test the sensitivity of the beamlets to aberrations. The effect of a minimum amount of spherical aberration on the beamlets proved to be severe, as 
the distorted wavefront produced considerable spot displacements in the sample plane. The high sensitivity attributed to the close proximity of the beamlets, leads to significant signal degradation (Figure 13).

In the Tscherning configuration, the reference mirror serves to provide an environment free from sample aberrations. The positions of the reference spots are configured to act as origins for the spot displacement calculations. A Helium-Neon laser provides the illumination onto a highly reflective surface used to re-image the beamlets from the sample to the detection plane. Adequate alignment is performed by certifying that the sample and reference mirror focal positions overlap. The reference mirror is then blocked out and the response of the lenslet array to aberrations was tested. One of the most common aberrations in biological samples is spherical aberration. The collar correction of the objective was incrementally increased by $0.01 \mathrm{~mm}$ (0.1 on the objective's scale and corresponding to a calibrated shift in the focal plane), inducing spherical aberration. The high sensitivity attributed to the close proximity of the beamlets, $3.75 \mu \mathrm{m}$ and $41 \mu \mathrm{m}$ in the sample and detection plane respectively, led to significant signal degradation due to astigmatism (Figure 13 and zoomed Figure 14). 

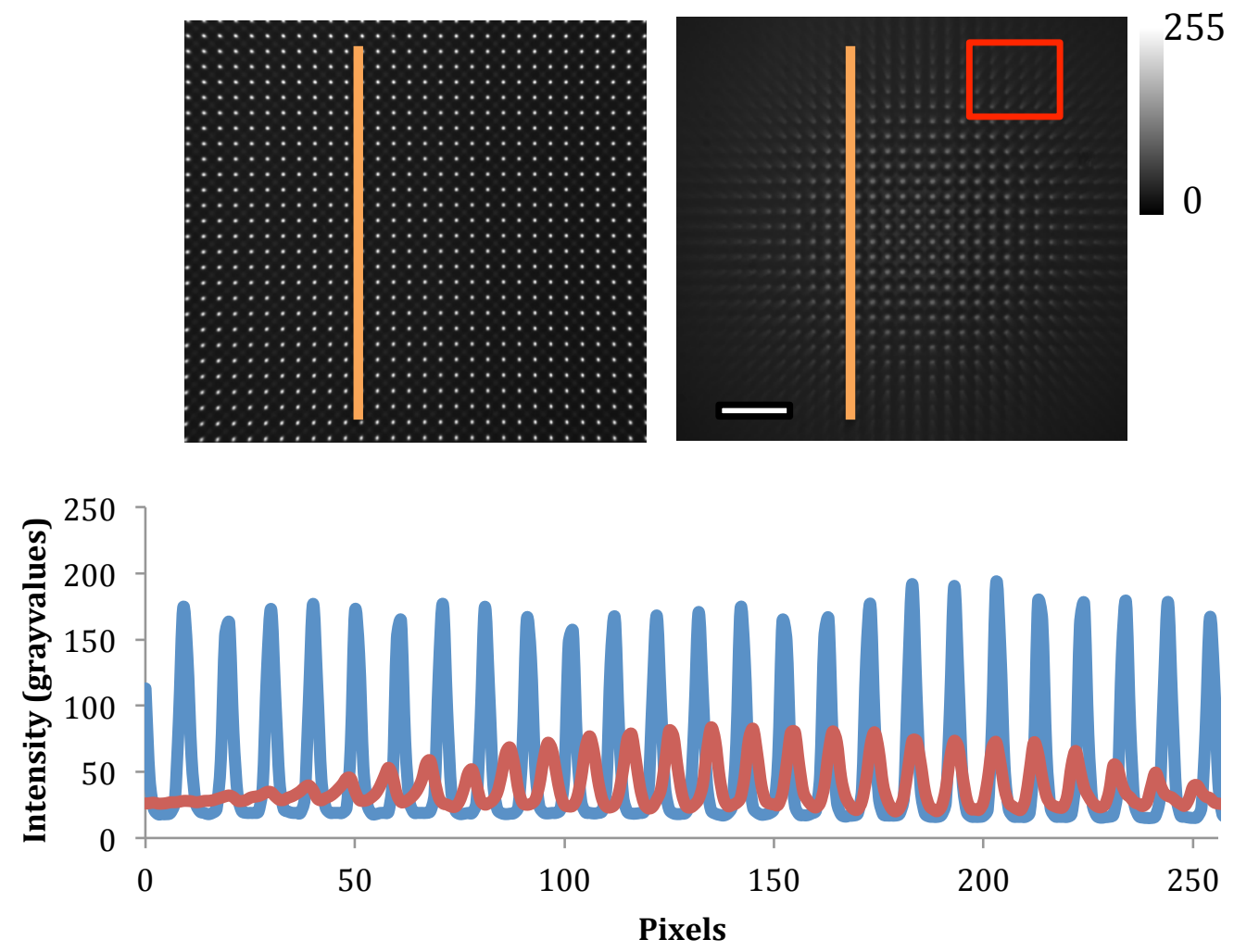

Figure 13: Effects of spherical aberration induced by $0.01 \mathrm{~mm}$ spherical aberration on the multifocal array generated via a microlens array in a TWS configuration. An aberration free environment (left) with spherical aberration (right) demonstrates high sensitivity to aberrations. Comparative line profile (orange) between nonaberrated (blue curve) and aberrated (red curve) shown below. Scale bar $100 \mu \mathrm{m}$.

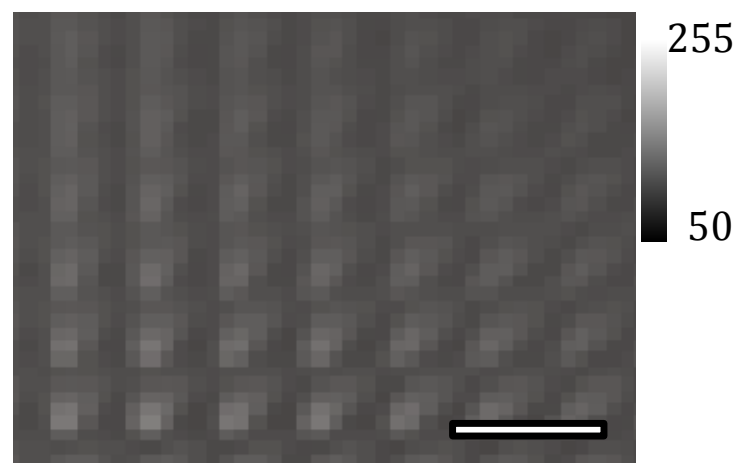

Figure 14: Expanded area of the beams in the region indicated by the red square in

Figure 13. It is clear that the high sensitivity of the beamlets to a variation in refractive indexes resulted in significant degradation of intensity. The comet tails 
displayed reduces centre-of-mass accuracy and increases possibility of crosstalk between neighbouring focal spots. Scale bar $50 \mu \mathrm{m}$. Image contrast altered to aid visualization.

In order to model the aberrations detected via the TWS, a zonal reconstruction (Appendix A) was developed to analyse a $49 \times 49$ portion of the retro-reflected beamlets. The wavefront was then fit using Zernike polynomials. However, the high sensitivity of the beamlets to variation in spherical aberration resulted in significant degradation of intensity and a sharp increase in crosstalk between sub-regions, severely limiting the dynamic range of a reliable reconstruction.

\section{Coefficients measured for various Zernike modes}

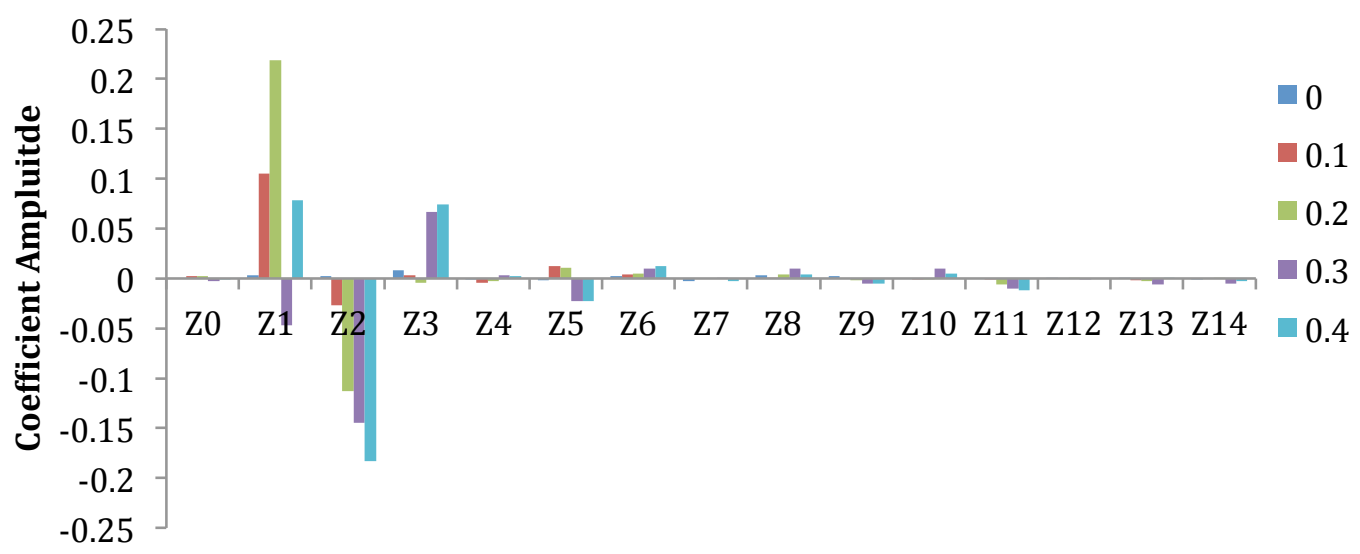

Figure 15: Spherical aberration coefficient determined vs. the various objective intervals, which provoke the spherical aberrations. In all cases the defocus was set to a minimum before determining the remaining coefficients.

As can be seen in Figure 15, a significant portion of largely irrelevant aberrations due to optical misalignment of the system, such as tip/tilt, is present in all measurements. The expected spherical aberration introduced has not been successfully reproduced in the reconstruction, expected in the presence of such intense crosstalk.

In order to establish a comparison between the TWS approach and established methods of aberration determination in microscopy; the setup was redesigned to incorporate a Shack-Hartmann WFS to test the aberrations as a function of induced 
spherical aberration. The lenslet array was removed and the expansion optics altered to adequately illuminate the sensor. As in the TWS, altering the collar correction on the objective in sequential 0.1 increments corresponds to an incremental introduction spherical aberration.

\section{Spherical Aberation Coefficient}

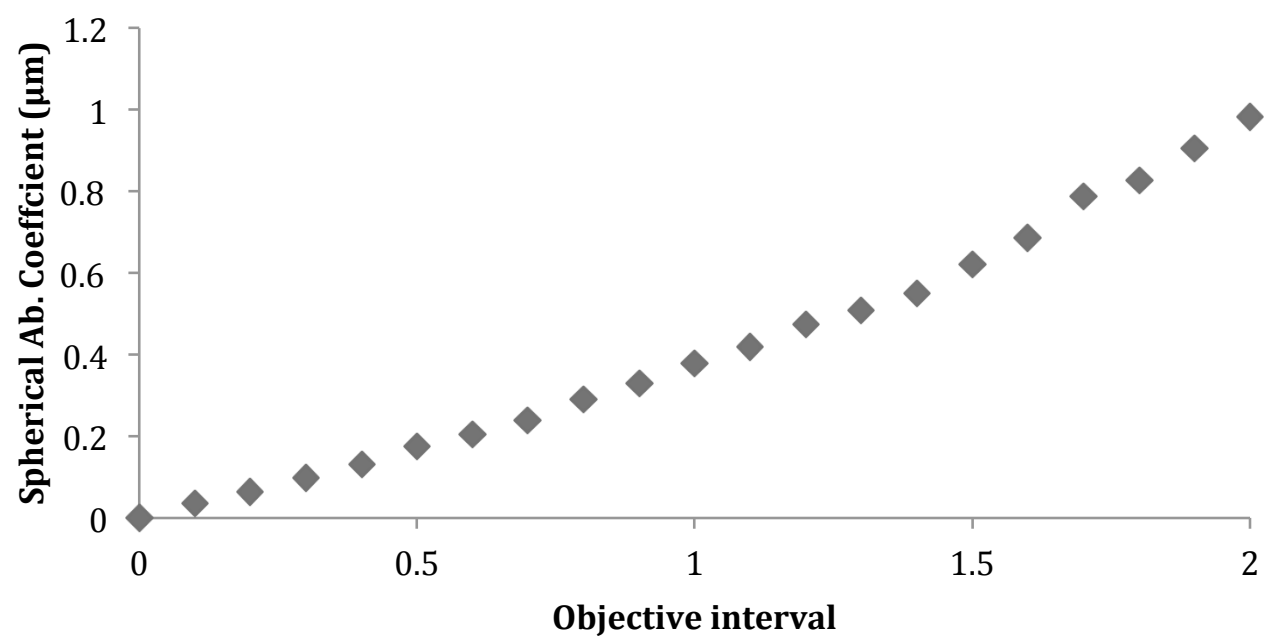

Figure 16: Spherical aberration coefficient determined vs. the incremental objective intervals inducing spherical aberrations. In all cases the defocus was set to a minimum before determining the remaining coefficients. As expected, throughout the experiment it was noted that the spherical term gained increased importance.

Minimal crosstalk (between sensing apertures) using the Shack-Hartmann wavefront sensor permitted the adequate detection of spherical aberration induced by the correction collar (Figure 16). Common to both wavefront-sensing approaches, the ROI selected does not cover the entire focal array generated from the microlenses. This effect may lead to underestimation of the coefficients, an issue which can be minimized with adequate boundary conditions or iterative approximations (Dai, 2008).

The dynamic range of the Tscherning WFS makes the approach successful in ophthalmology. A typical retina of 6-7 $\mathrm{mm}$ in diameter exhibits significantly larger projection area for the beamlets. Using an approximation to a circular area; a $10 \times 10$ Tscherning screen projected to fully illuminate the retina produces 
approximately $0.28 \mathrm{~mm}$ pitch. Therefore the noteworthy separation significantly reduces the effect of crosstalk, while providing sufficient sensitivity for ophthalmology (Harmening et al., 2007; Kaemmerer et al., 2000). Indeed, if the proposed objective for the MegaFLI had significantly lower NA then the comet tails would be less evident.

Overlapping comet tails proved a main obstacle in the system design, consequence of the significant sensitivity of the TWS. In a situation with a fluorescent sample, two-photon induced fluorescence from the tails would generate out-of-focus fluorescence in the detector plane. Even under the assumption that the system is an aberration free environment, it would be challenging to effectively align the fluorescence generated from the microlens array onto the SPAD. The TWS does not provide sufficient flexibility to individually manipulate the excitation volumes. The SPAD has a low fill factor and the active areas of the detectors have separation variations of $<1 \mu \mathrm{m}$; deviations result of the manufacturing process (Tyndall et al., 2011). The likelihood of accurate alignment of the beamlets to the minor variations in separation added to need to correct for rotational, tip and tilt variations of the detector once assembled on the optical table, would be technically challenging to achieve $100 \%$ fill factor for the total amount of illuminated detectors. However, minor system aberration is unavoidable, making the Tscherning approach for multifocal FLIM on such small active areas technically exigent.

Regarding future prospects of the TWS, there is still scope to develop this WFS for biological microscopy. Under a different optical configuration, a SLM could be placed in the back plane of the lenslet array, generating therefore a closed loop adaptive optical system. Furthermore, with a blaze function on the SLM it becomes viable to selectively illuminate the back of the microlenses, recruiting illuminated beamlets. This can provide a selective beam scanning approach, which has the possibility to minimize optical crosstalk. Unfortunately, these configurations are beyond the scope of this thesis. 

MODULATOR

Two further methods for beamlet generation were investigated using SLM technology in phase only configuration. This section discusses two SLM systems that were used, calibration procedures and operating methodology and outlines two approaches to beamlet generation: Huygens-Fresnel diffractive lenses and holographic beam generation.

A spatial light modulator (SLM) is an optical device that can modulate the amplitude, phase or polarization of light, or a combination of these. In microscopy these devices have been used extensively in STED (Gould et al., 2012; Lenz et al., 2011), holographic optical tweezers (Schonbrun et al., 2005), adaptive optics (Neil et al., 2000b), structured illumination (Débarre et al., 2008; Kner et al., 2009) and pulse shaping (Weiner, 2000).

A reflective SLM configuration consists of a liquid crystal on silicon layer (LCOS) based on electrically controlled birefringence (ECB). A nematic liquid crystal orientation is aligned by means of a surfactant orientation layer, and the bulk alignment is modulated by dielectric coupling with an electric field applied between the two electrodes. ECB mode uses the applied voltage to change the tilt of the liquid crystal molecules in the direction of the electric field; changing the birefringence as a function of the tilt angle, resulting in a modulation of the spatial properties of an incoming beam (Armitage et al., 2006; Hermerschmidt et al., 2007; Osten et al., 2007). A LCOS is a reflective microdisplay based on a silicon backplane. The silicon chip is coated with a highly reflective surface and contains the electronic circuits responsible for controlling the liquid crystals. As the circuits are located behind the pixel this provides a high fill factor and avoids obstructions in the light path (compared with transmissive LC in which the electronics surround the pixels). The LCOS works as an extended monitor, where all phase functions (gratings, phase masks, holograms, etc.) are addressed via a standard Digital Visual Interface (DVI) (Proll et al., 2004). A phase mask is projected on the microdisplay, which modulates the wavefront of the incident light on a pixel-by-pixel basis (Shrauger and Warde, 
2001). The phase mask corresponds to the Fourier transform of the desired intensity distribution. The ability of the SLM to represent complex-valued images enables intensive information processing functions using the Fourier transform properties of light (Cohn, 2001). A comparison between various commercially available reflective phase only SLMs is shown in Table 4.

\begin{tabular}{|c|c|c|c|c|c|}
\hline & $\begin{array}{l}\text { Display } \\
\text { Size } \\
\text { (pixels) }\end{array}$ & $\begin{array}{l}\text { Pixel } \\
\text { Pitch } \\
(\mu \mathrm{m})\end{array}$ & $\begin{array}{c}\text { Fill } \\
\text { Factor } \\
(\%)\end{array}$ & $\begin{array}{c}\text { Refresh Rate } \\
(\mathrm{Hz})\end{array}$ & $\begin{array}{c}\text { Microdisplay } \\
\text { Flatness }(\lambda)\end{array}$ \\
\hline $\begin{array}{c}\text { Boulder } \\
\text { Nonlinear } \\
\text { Systems } \\
\text { HSPDM } 512\end{array}$ & $512 \times 512$ & 15 & 100 & 100 & 0.25 \\
\hline $\begin{array}{c}\text { Holoeye } \\
\text { Pluto NIR2 }\end{array}$ & $1920 \times 1080$ & 8 & 87 & 60 & 3 \\
\hline $\begin{array}{c}\text { Hamamatsu } \\
\text { X10468 }\end{array}$ & $800 \times 600$ & 20 & 95 & 60 & $0.025^{*}$ \\
\hline
\end{tabular}

*Sold with added corrective phase pattern

Table 4: Comparison between various commercially available reflective phase only SLMs

\subsection{SPATIAL LIGHT MODULATOR WAVELENGTH CALIBRATION}

Prior to implementing any diffractive pattern on to the SLM microdisplay it is necessary to adequately calibrate for the excitation wavelength to guarantee $0-2 \pi$ phase modulation (Osten et al., 2007). Throughout the project, two SLM devices were used: Holoeye Pluto NIR2 and the Boulder HSPDM512 SLM. In the first instance, the Holoeye Pluto NIR2 was used for the Huygens-Fresnel approach 
(Section 10.4). Due to significantly lower microdisplay aberration, higher dynamic range and $100 \%$ fill factor the Boulder HSPDM512 replaced the Holoeye Pluto NIR2 and used in holographic approach and throughout the experimental results (Section 10.6 and Section 14).

The SLM has a wavelength dependency (Liu et al., 2006; Osten et al., 2007). The parallel alignment of the LC molecules undergoes a tilt, which induces a refractive index variation when light is reflected. It becomes necessary to ensure that the SLM can guarantee adequate phase modulation between $0-2 \pi$, which is accomplished with suitable calibration for a range of incident wavelengths on the LCOS-SLM display.

The LCOS-SLM's has a wavelength range. A gamma curve calibration ensures adequate $0-2 \pi$ phase modulation. The gamma curve correlates the SLM input (8bit: 0-255, 16bit: 0-65536) and the output optical phase shift (up to $2 \pi$, or more). Various calibration approaches include a Michelson interferometer (Hendriks et al., 2012), slit interferometric phase measurement (Bergeron et al., 1995) or a variable checkerboard phase pattern (Luberek, 2010).

Various SLM manufactures recommend different calibration procedures for their devices. As such, the Holoeye Pluto NIR2 was calibrated via an interferometric measurement (Hermerschmidt et al., 2007) and the Boulder HSPDM512 using the variable checkerboard pattern. The procedures are based on monitoring the zeroth order intensity variation as a function of phase induced by the SLM. Stepping segments of the SLM while maintaining the remaining parts constant at the maximum phase value, results in a zeroth-order spot intensity variation in agreement with $\frac{1+\cos \theta}{2}$ where $\theta$ is the phase contrast. As such, maximum intensity is achieved at $\theta=2 \pi$ and the diffraction loss into higher orders is maximized when the phase contrast equals $\pi$.

Since the phase retardation introduced by a liquid crystal display is dependent on both voltage and illumination wavelength, adequate calibration of the SLM at each operating wavelength ensures suitable $0-2 \pi$ phase modulation range (applied 
voltage range). The first calibration procedure used with the Holoeye Pluto NIR2 was an interferometric measurement of the phase modulation. An expanded and collimated beam is partially transmitted through a double-pinhole aperture, incident on two separate sections of the LCOS (Figure 17). Each beam generated from the mask illuminates a partial area in each half of the display, which is addressed with a phase pattern. Throughout the experiment one half of the microdisplay remains constant while the second is addressed with a variable grey level (0-255). The beams incident on the SLM interfere due to a telescope located in the zeroth order diffraction and captured by a CMOS camera. One line of interference pattern is registered at every iteration of the grey level variation (Figure 17). The shift of the interference pattern position is directly proportional to the phase shift created by the incremental variations on one half-screen of the SLM (Hermerschmidt et al., 2007). The interference pattern registered by Holoeye's Phase Cam acquisition software determines the linear phase region (Figure 17) and calculates the corresponding gamma curve (Figure 18), therefore generating a linear relation between $0-2 \pi$ (phase) and 0-255 (gray values).

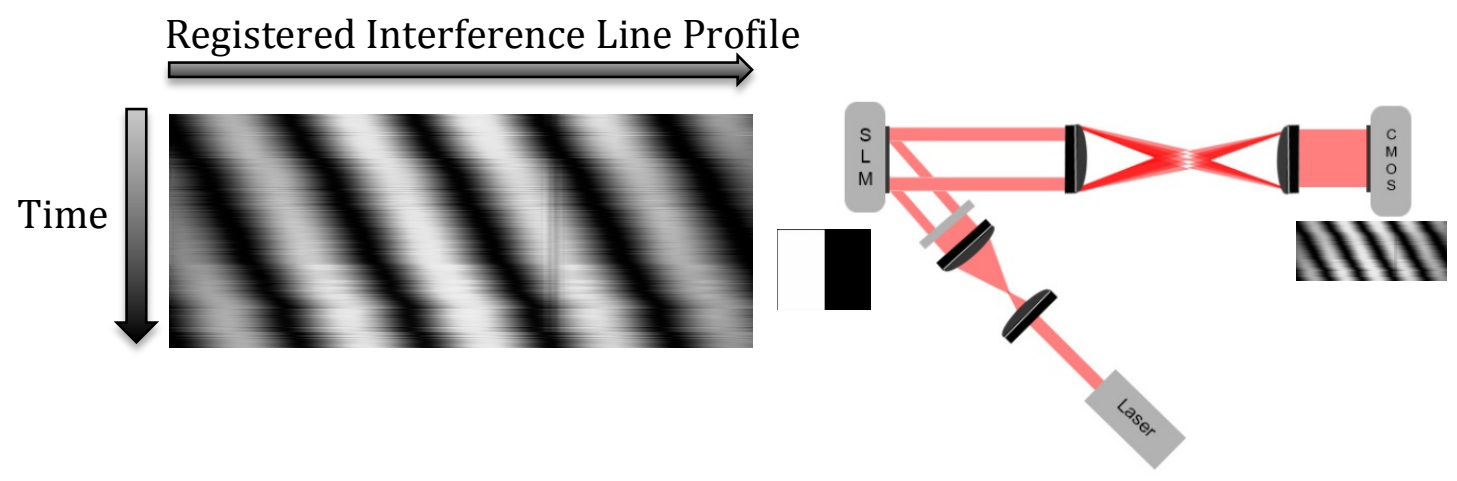

Figure 17: Interference pattern (left) registered at every iteration of the grey level variation was acquired using a double pinhole calibration setup (right). The pattern demonstrates a linear relationship between $0-2 \pi$ over the $0-255$ grey level range projected on the microdisplay. 


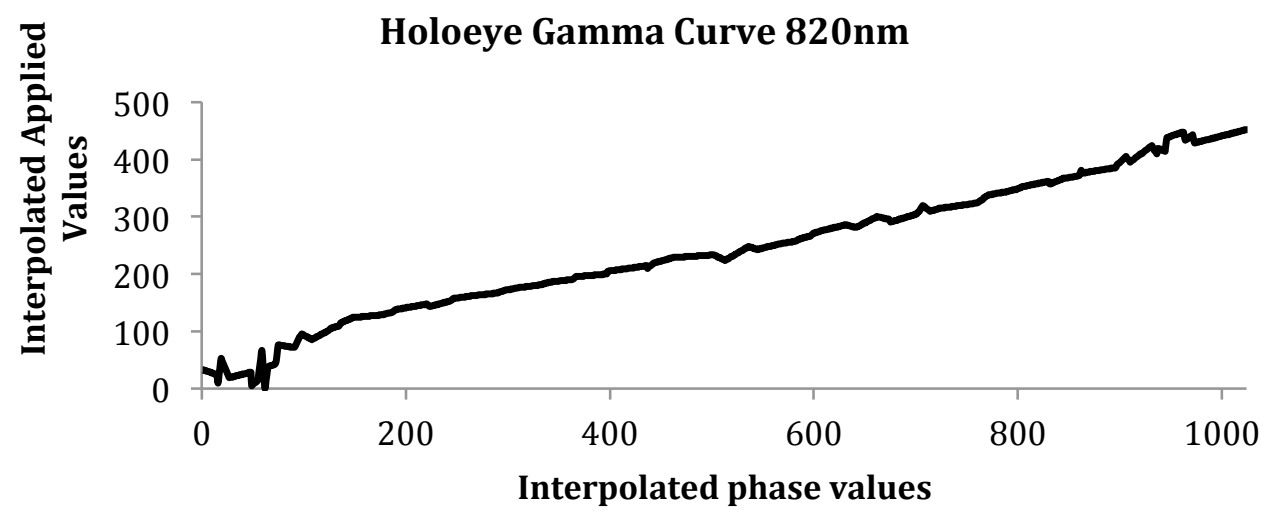

Figure 18: The linear relationship between $0-2 \pi$ is due to the gamma curve calibration set on the SLM firmware.

For the Boulder HSPDM512 SLM a checkerboard pattern calibration procedure was developed. This approach does not require the incoming excitation on the SLM to be divided. Instead the fully illuminated LCOS displays a checkerboard pattern in which the alternating squares represent the maximum (65000) and minimum phase value. Throughout the procedure the squares, which display the minimum value, are iteratively stepped to match the maximum. Each step of the iteration the maximum intensity of the zeroth order central spot focused onto a camera is registered. A CMOS camera located in the Fourier plane of the SLM $\left(I_{5}\right)$ (Figure 17) sequentially registers a variation of the zeroth order spot intensity. Correct gamma curve (Figure 19) produces zeroth order intensity variation in agreement with $\frac{1+\cos \theta}{2}$ (Figure 20). 


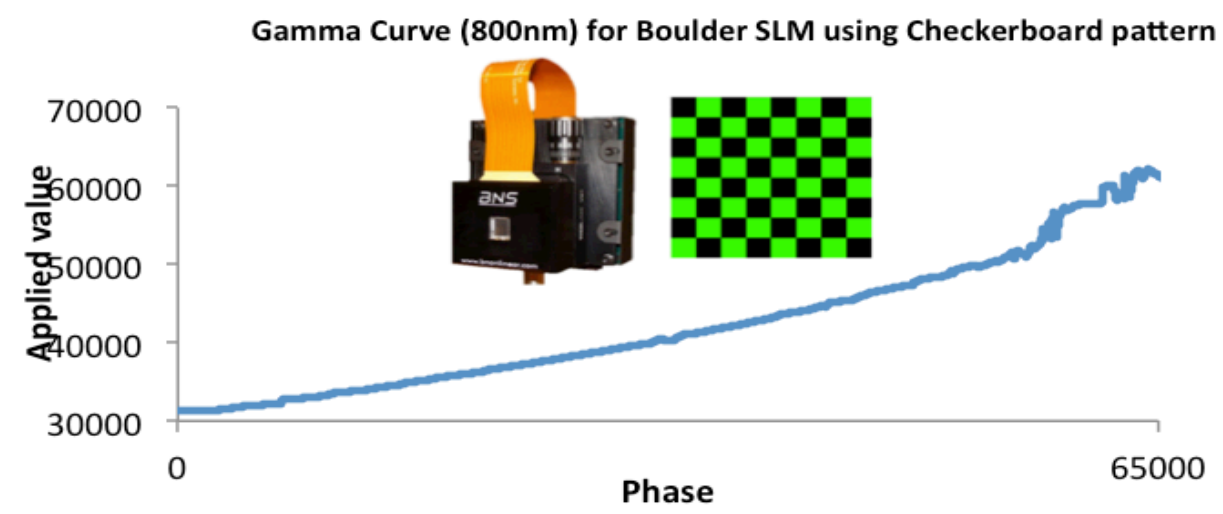

Figure 19: Calibrated gamma curve for $800 \mathrm{~nm}$ produced via the checkerboard pattern exemplified in black and green.

0

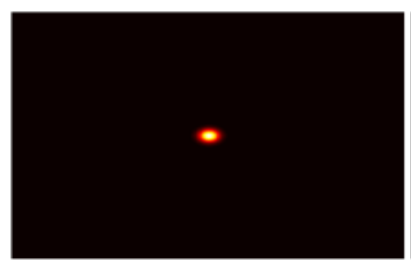

$\pi$

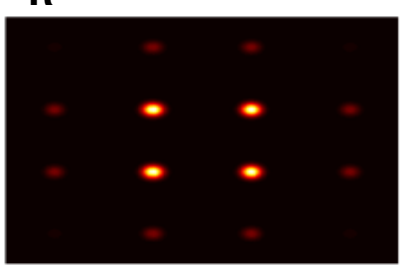

$\pi / 2$

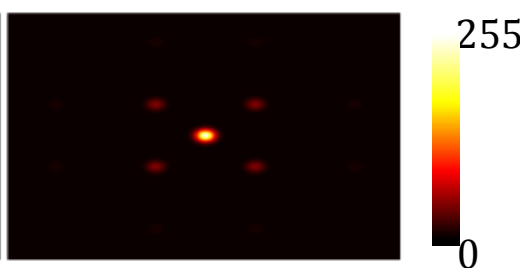

$3 \pi / 2$

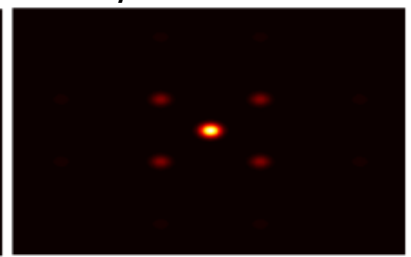

Figure 20: Boulder HSPDM512 SLM wavelength calibration using the checkerboard pattern approach. A calibrated gamma curve produces zeroth order spot intensity in agreement with $(1+\cos \theta) / 2$, as shown by the images presented. Scale bar 200 $\mu \mathrm{m}$.

\subsection{HUYGENS-FRESNEL DIFFRACTIVE LENS ARRAY}

As an alternative to refractive lenses, a diffractive lens arrangement was considered. Fresnel phase patterns projected using an SLM generates a flexible multifocal environment with the potential of incorporating Zernike aberration correction directly to each lens component (Colyer et al., 2011; Goodman, 1996; 
Tyndall et al., 2011). The Fresnel approach requires dedicated segments of the diffractive pattern for the generation of the respective beamlets.

The Huygens-Fresnel principle, used to generate the beamlets can be stated as:

$$
U\left(P_{0}\right)=\frac{1}{i \lambda} \iint U\left(P_{1}\right) \frac{e^{i k r_{01}}}{r_{01}} \cos \theta d s
$$

Where $U\left(P_{0}\right)$ is the complex amplitude at $P_{0}, \theta$ is the angle between the outward normal $\mathrm{n}$ and $r_{01}$ the vector (vector between $P_{0}$ and $P_{1}$ ). The Fresnel approximation simplifies the expression to:

$$
r_{01}=z\left[1+\frac{1}{2}\left(\frac{x-x_{0}}{z}\right)^{2}+\frac{1}{2}\left(\frac{y-y_{0}}{z}\right)^{2}\right]
$$

It is obtained by considering the distance $r_{01}$ between $P_{1}$ and $P_{0}$ and using a binomial expansion while retaining the first three terms (Hu et al., 2009).

The Huygens-Fresnel principle considers each point of a wavefront as a source of outgoing secondary spherical waves. Modifying light rays with an appropriate phase shift creates a multibeam pattern at the intermediate focal plane, result of the constructive and destructive interference induced (Wang et al., 2005).

The phase pattern projected on the SLM was calculated in accordance with the Fresnel approximation (Eq. 36). The amount of beamlets generated is proportional to the sum of segments projected on the microdisplay (Figure 21). The images demonstrated in Figure 21 were acquired using a correction for the SLM microdisplay flatness (Figure 23). An example of $2 \times 2$ and $4 \times 4$ beamlets projected into an intermediate image plane in the optical train is given in Figure 21. 
A

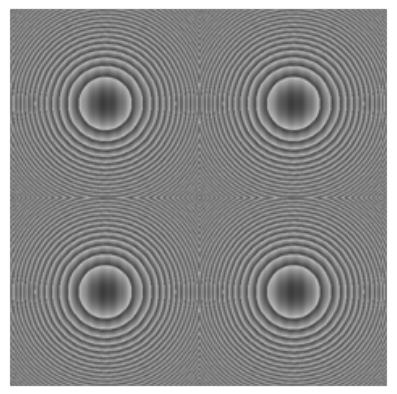

C

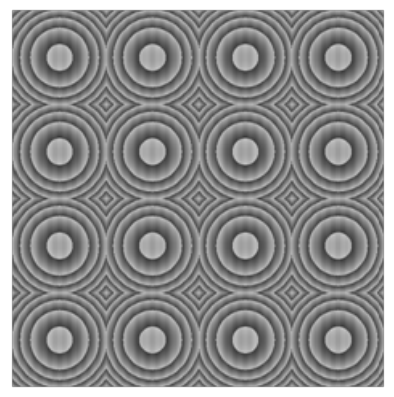

B

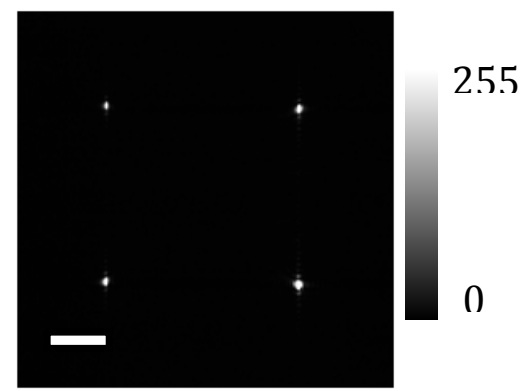

D

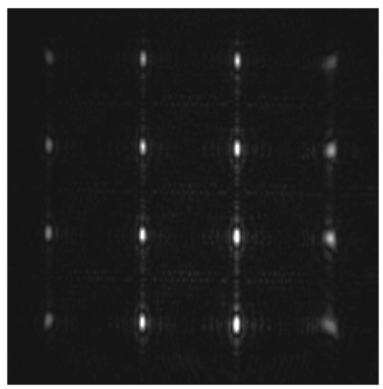

Figure 21: Projected Fresnel lenses on the Holoeye Pluto NIR2 SLM microdisplay with the added blaze function ( $\mathrm{A}$ and $\mathrm{C}$ ) and the result at the intermediate focal plane ( $B$ and $D$ ). $A / B$ and $C / D$ represent a $2 \times 2$ and $4 \times 4$ array respectively. Images acquired using a CMOS camera with AO correction for SLM microdisplay. Scale bar $100 \mu \mathrm{m}$.

Generation of Fresnel lenses based on the Huygens-Fresnel principle has considerable benefits over the microlens array (Colyer et al., 2011). The possibility of generating a flexible number of beamlets in an array with variable positions permits alignment of sample fluorescence onto the individual SPADs, in addition to adaptive optical correction.

Illustrated in Figure 21 is the segmentation of the hologram space in order to generate beamlets. Pixel density on the LCOS display provides a limiting factor in the amount of beamlets that can be produced for a given effective NA. The Holoeye Pluto NIR2 serves to be the most adequate SLM for this application as it has a 1920 × 1080 pixel display. However, unlike other SLM models the $3 \lambda$ LCOS flatness from the microdisplay results in high phase distortion of the projected pattern (Table 4). 


\subsection{RANDOM SEARCH ALGORITHM}

Due to the $3 \lambda$ flatness of the Holoeye Pluto NIR2 SLM micro-display, an adaptive optical correction was implemented. The adaptive optical correction, based on the addition of Zernike polynomials to the hologram, was performed using a Random Search (RS) optimization. In the context of biological imaging the RS algorithm was first implemented in confocal microscopy using DMM (Wright et al., 2005). A DMM actuator is randomly selected and perturbed with an indiscriminate level. The signal acquired by the PMT then evaluates the effectiveness of the varied voltage applied. This iteration is performed over a user-selected amount of cycles or a programmable stopping parameter (for example no significant improvement after $\mathrm{x}$ iterations). Once the algorithm terminates, the laser beam, expanded in order to overfill the DMM, would have a wavefront capable of counteracting the refractiveindex variations provided by the sample (Wright et al., 2005). The random approach does reduce the algorithms effectiveness at searching for a truly global solution but converges on a solution relatively quickly. The optimization routine was developed to find a rapid answer to the vast solution space provided by the Boston Micromachines Micro DMM; as perturbing each of the 37 actuators individually over the 0-200 $\mathrm{V}$ dynamic range, provides for $200^{37}$ possible solutions. The RS algorithm is characterized as simulated annealing. Simulated annealing is an optimization strategy that simulates the characteristics of the process of crystallization of a slowly cooled liquid that results in a perfect crystal structure as a result of the slow progression. In analogy to crystallization, the quality improves as a function of time. The algorithm is designed on accepting improvements throughout the process, maximizing on a given parameter, in this case intensity. Therefore simulated annealing algorithms are a compromise between the time it takes to run the algorithm and the quality of the resulting image. As such it is not suitable for interactive use, but is recommended if calculation speed is not an issue (Di Leonardo et al., 2007; Spalding et al., 2008).

Figure 22 explains the algorithm's optimization procedure using a SLM. This particular algorithm applies a series of Zernike polynomials with various amplitudes at random, while registering the appropriate maximum intensity. After a predefined 
series of iterations the algorithm displays the corrected Zernike phase pattern. This pattern corresponds to a summation of individual Zernike modes with the respective coefficients related to the maximum intensity registered by a camera positioned in the immediate focal plane. Figure 23 represents the significant improvement obtained in intermediate focal plane, although some residual aberrations remain.
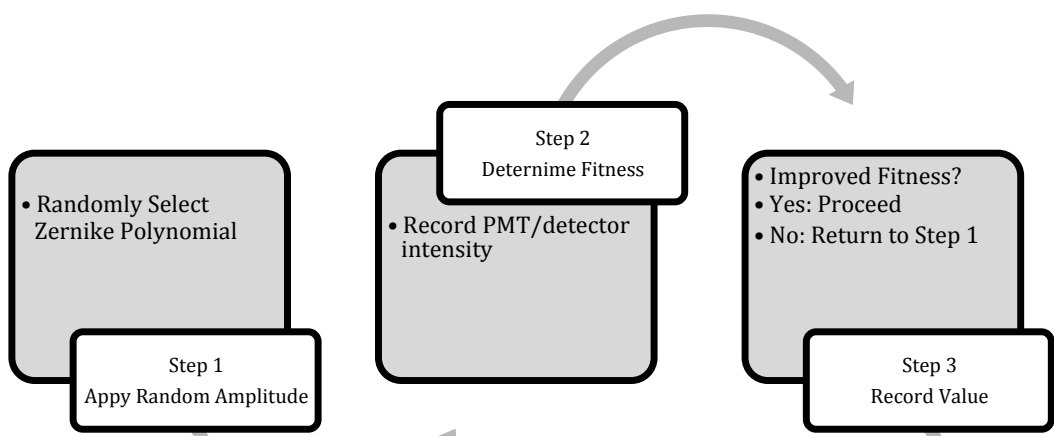

Step 4 Solution: Apply Pattern

- Have the mprovements stagnated? - Yes : Solution - No: Step 1

Figure 22: Schematic of RS optimization adapted for the SLM using Zernike polynomials. The algorithm consists of applying random Zernike coefficient amplitudes and registering the intensity. Coefficients corresponding to improvement are recorded. Once improvements stagnate the solution is found. 


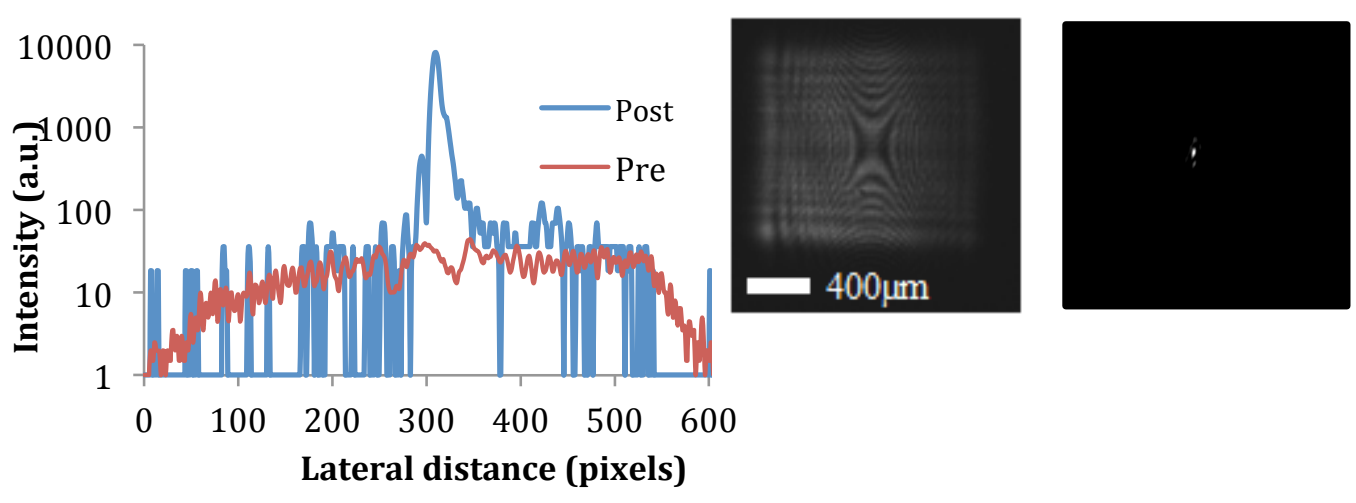

Figure 23: Laser focal spot signal improvement obtained in the Fourier plane the SLM performed by the RS optimization using Zernike polynomials. The preoptimized (A) is shown in comparison with the post-optimized obtained (B). Despite significant improvement B still shows residual aberrations. Overexposure is deliberate to enhance the asymmetry of low intensity features.

This correction developed for Fresnel lenses shows a significant improvement on the overall wavefront for all beamlets, illustrated in Figure 24.
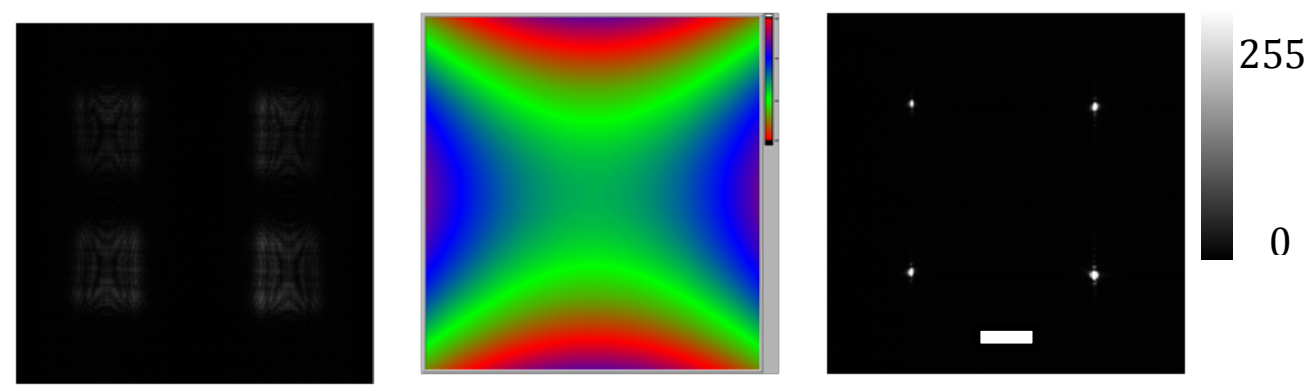

Figure 24: Original immediate focal plane of the Fresnel pattern projection (left) demonstrates significant improvement (right) after application of RS-AO pattern (middle) Scale bar $100 \mu \mathrm{m}$.

As the various regions of the micro-display suffer from different distortions due to the manufacturing process of the SLM, segmentation of the hologram for lenslet generation requires that we produce individual correction on each of the beamlets. Individual Zernike patterns were generated for each spot and then added to the corresponding Fresnel lens. Demonstrated in Figure 25 is the manipulation of 
individual focal spots. Different distortions were added with various coefficients to a $2 \times 2$ beamlet array.

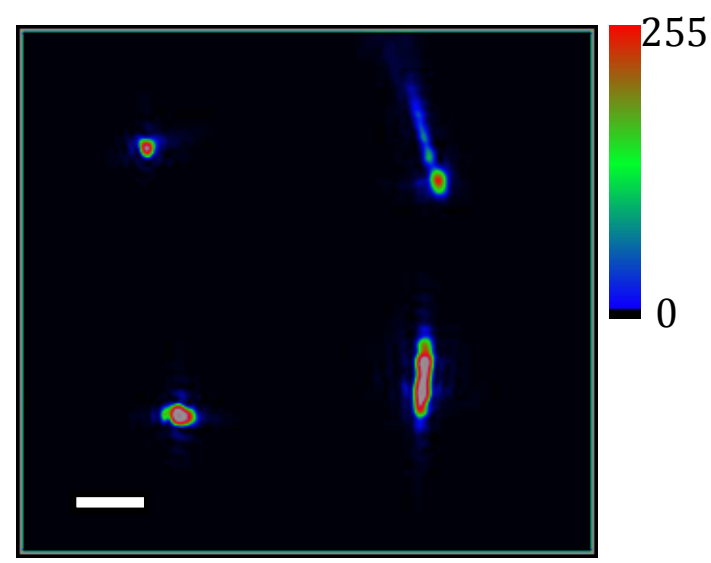

Figure 25: Fresnel lenses with variable aberration applied. Overexposure is deliberate to enhance the asymmetry of low intensity features. The first spot is the result of system corrections (top left), the second (clockwise) has added spherical aberration, the third has added defocus and astigmatism and the forth was individually optimized for all. Scale bar $100 \mu \mathrm{m}$.

Segmentation of the hologram space into squares, in which each one generates a focal volume, introduces the need for individual correction of each beamlet to account for the local variation in that segment. An increase in the number of beamlets generated proportionally requires appropriate corrections.

In addition to optical aberration, the beamlets have significant variation in intensities due to the Gaussian profile of the excitation beam expanded to illuminate the SLM. This results in intensity variations that cannot be compensated easily using this multifocal generation approach. Expanding the illumination onto the SLM creates a more uniform intensity distribution, however limits the photon density in each area. As the generation of beamlets using Fresnel lenses occur due to a segmentation procedure, the back focal plane of the imaging objective undergoes segmentation itself. Throughout the imaging system the beamlets propagate 'independently', as these are separated from each other from the SLM onwards. 
In summary, the beamlets produced are the result of a calculated projection onto a SLM, which provides increased flexibility as it becomes possible to manipulate the positions of the excitation volumes in $2 \mathrm{D} / 3 \mathrm{D}$ via Zernike polynomials; however concerns arise regarding the amount of viable beamlets that can be produced. 


\subsection{HOLOGRAPHIC MULTIFOCAL GENERATION (GERCHBERG-SAXTON} ALGORITHM)

The final optical solution to beamlet generation investigated in this thesis is holographic in nature. Holograms are now routinely produced using an iterative algorithm, which converges on a phase representation of a desired pattern. The Gerchberg-Saxton (GS) algorithm (Gerchberg and Saxton, 1972) occupies the entire hologram space, which is iteratively optimized to generate the desired intensity pattern in Fourier space of an SLM. The GS approach can produce an arbitrary pattern in two and three-dimensions (Whyte and Courtial, 2005). The GS algorithm has been used in pulse shaping (Ahn et al., 2003), electron microscopy (Gerchberg, 1972) and is particularly popular in holographic optical tweezing (Bianchi and Di Leonardo, 2010; Spalding et al., 2008). For holographic optical tweezers, the flexibility and rapid calculation makes it possible to trap various particles simultaneously and define their trajectory (Bowman et al., 2011; Leach et al., 2006). The hologram occupies the entire hologram space without segmentation: a correction over the entire surface serves to adequately compensate for system wavefront aberration.

Initially an arbitrary distribution, frequently null, is allocated to the initial phase distribution in the hologram plane $\left(\varphi_{0}^{H}\right)$.

$$
\varphi_{0}^{H}=0
$$

The GS algorithm (Figure 26) is based on the sequential calculation of the distribution in the hologram plane, $\varphi_{n}^{H}$, from the previously calculated phase distribution, $\varphi_{n-1}^{H}$. The following steps of each iteration then progresses as follows:

$$
\begin{array}{ll}
u_{n}^{H}=\sqrt{I_{H}} \exp \left(i \varphi_{n-1}^{H}\right) & 39 \\
\varphi_{n}^{T}=\arg \left(F F T\left(u_{n}^{H}\right)\right) & 40 \\
u_{n}^{T}=\sqrt{I_{T}} \exp \left(i \varphi_{n}^{T}\right) & 41 \\
\varphi_{n}^{H}=\arg \left(F F T^{-1}\left(u_{n}^{T}\right)\right) &
\end{array}
$$


Where $\varphi_{n}^{T}$ and $\varphi_{n}^{H}$ are the phase distribution in the target and hologram plane respectively, $I_{T}$ corresponds to the target intensity, given the field $u_{n}^{T}$. Sequential calculations of equations 39-42 provide the phase distribution in hologram plane corresponding to the desired target plane intensity distribution. After a series of successive approximations the intensity in the target plane, $I_{T}=\left|u_{n}^{T}\right|^{2}$, converges to an almost diffraction limited approximation of the desired intensity $I_{T}$. Convergence determination results in a stagnation of the improvement experimental determined or over a user-defined number of iterations. In the case of a single spot, the intensity in target plane, $I_{T}=\left|u_{n}^{T}\right|^{2}=1$. The intensity distribution in the hologram $\left(I_{H}\right)$ and Fourier plane $\left(I_{T}\right)$ are expressed as $x y$ functions, which are saved as 2D arrays of real-valued numbers (Whyte and Courtial, 2005).

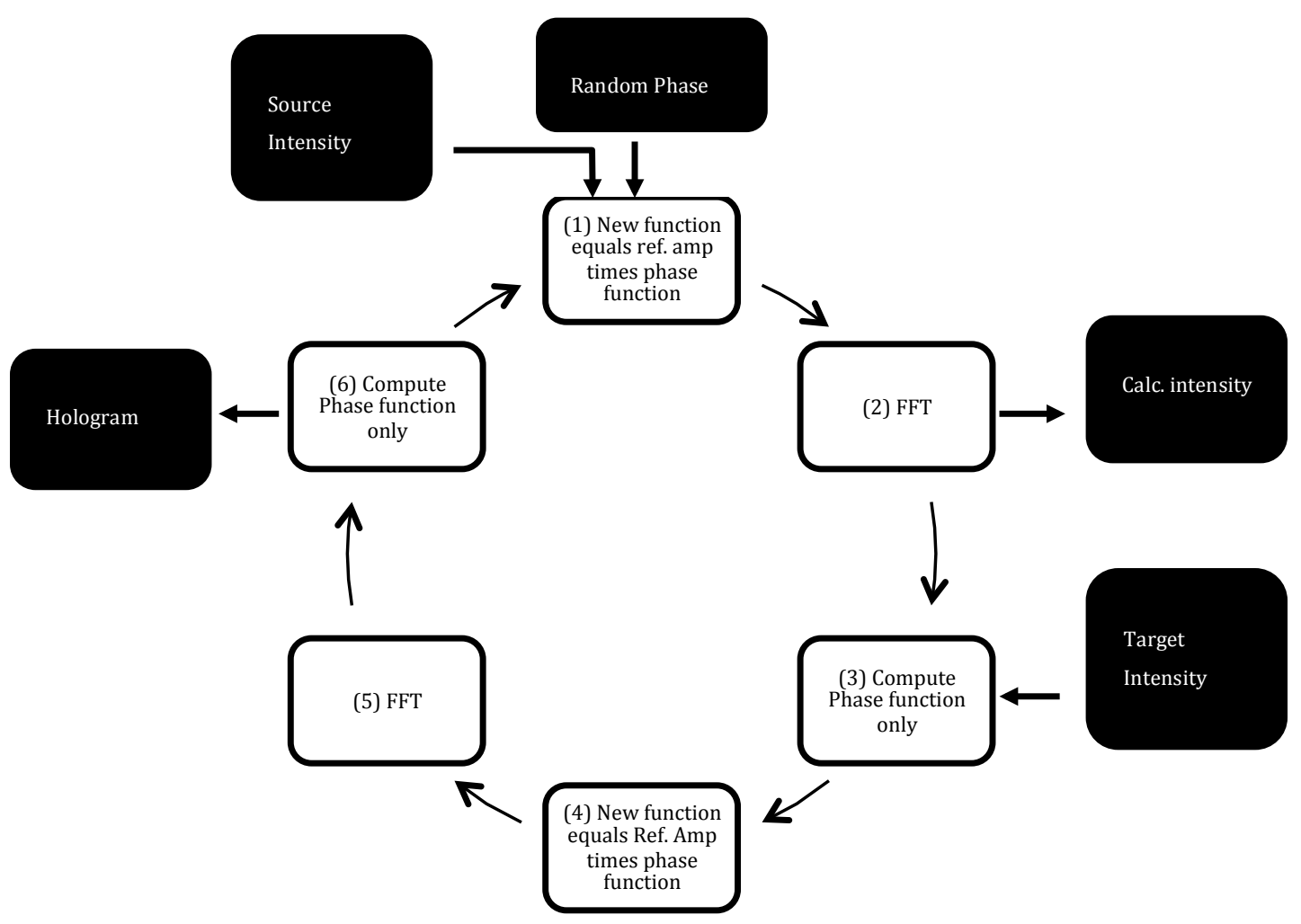

Figure 26: GS algorithm flow chart. An approximation to the desired target intensity results from sequential calculations (steps 2-5, Eq. 39-42) of the phase function, producing a hologram (step 6). The Random phase only takes part of the loop in the first iteration. 
The inflexibility from the refractive lenslet array and the adaptive optical correction necessary for the limited amount of beamlets produced by the segmented Fresnel lens approach, led to the implementation of the holographic projection of arbitrary beams.

The GS algorithm depends on an iterative calculation of the phase hologram of any desired pattern. Figure 27 demonstrates the iterative approach to calculating the desired diffraction pattern. Experimentally determined via successive monitoring of the Fourier and hologram plane, a minimum of 30 iterations is required for the GS algorithm to converge, for any desired pattern (Di Leonardo et al., 2007). Figure 28 and Figure 29 show focal spot pattern generation in the Fourier plane of the SLM.

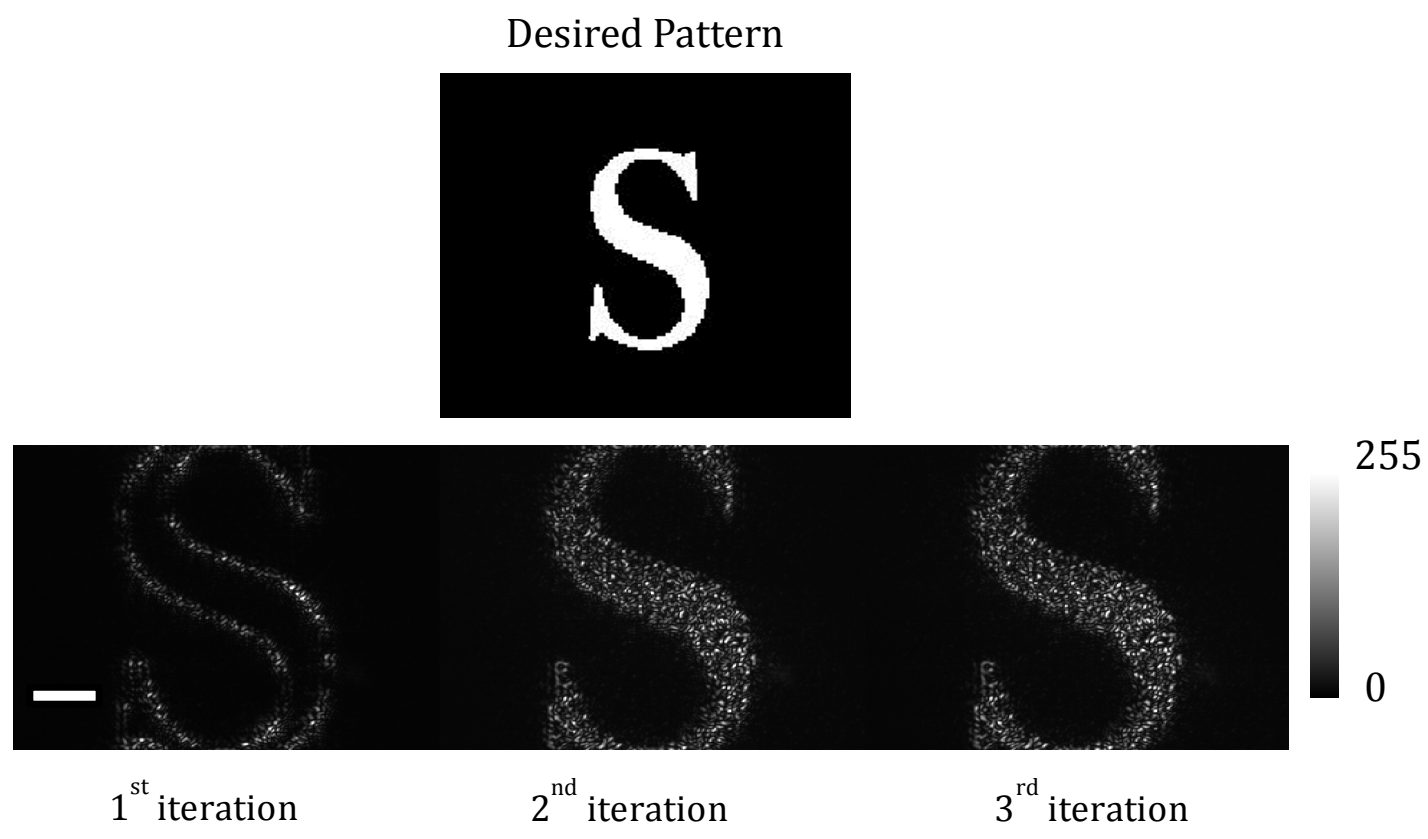

Figure 27: Demonstration of the iterative nature of the GS algorithm. Images acquired by Thorlabs CMOS camera in the Fourier plane of the SLM. Scale bar 100 $\mu \mathrm{m}$. The first iteration of the desired pattern shows only the contours of the desired intensity pattern. As the algorithm progresses a more accurate approximate is attained, as the full desired target pattern is evident with a higher intensity. The full 30 iterations have duration of approximately 2 minutes. 

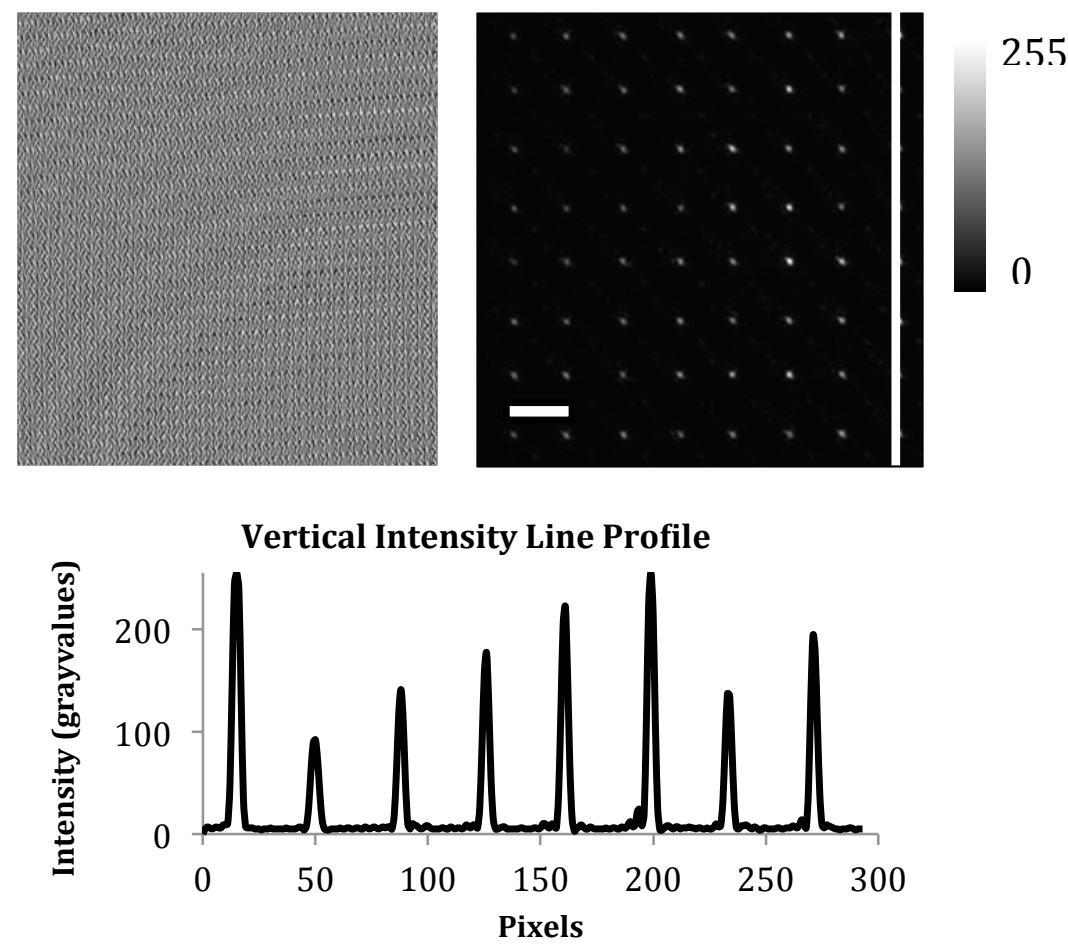

Figure 28: GS phase projection in the Hologram plane and resulting focal plane detected by a CMOS camera. Vertical line profile shows the variations in intensity throughout the beamlet array. Scale bar $100 \mu \mathrm{m}$.
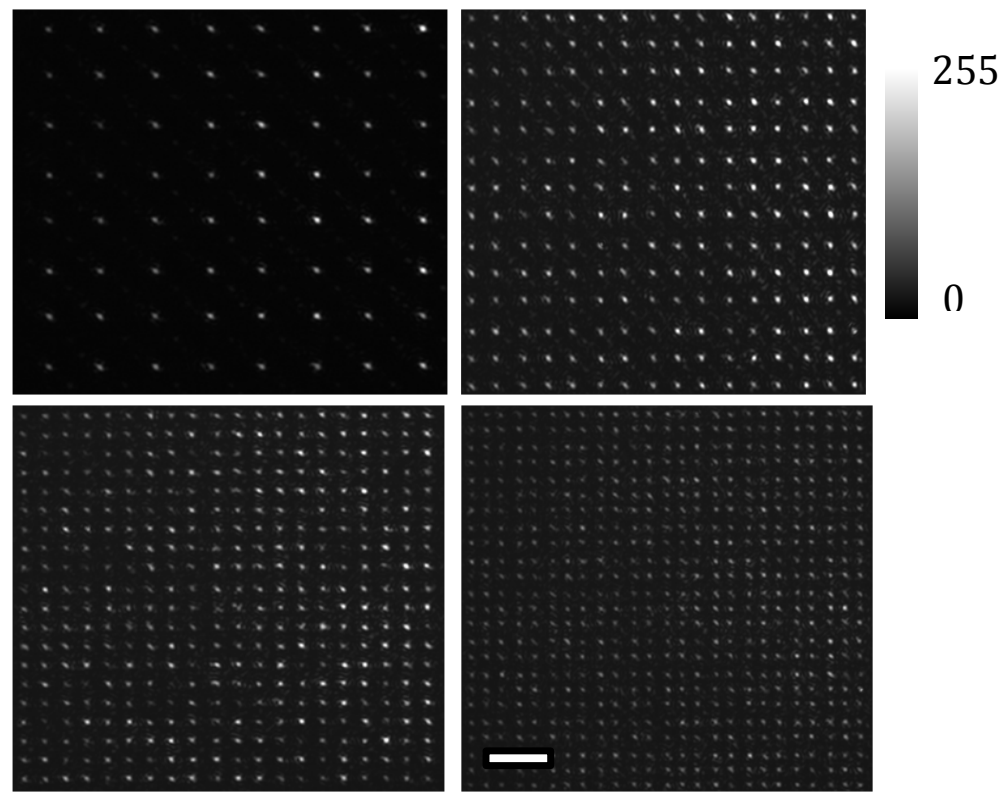

Figure 29: Generation of a series of focal volumes captured by the CMOS camera in the focal plane. The total amount of beamlets generated (top to bottom, left to right) $64,256,400$ and 625. Scale bar $100 \mu \mathrm{m}$. 
The standard GS-algorithm produces intensity variations throughout the beamlet array (Figure 28 - line profile). Albeit the variation has no easily identifiable pattern. The variation in uniformity is approximately $60 \%$, a standard value for the GS algorithm (Di Leonardo et al., 2007; Spalding et al., 2008). These intensity differences generate variable illumination onto the sample and subsequently onto the SPAD array. To counteract this, a weighted GS algorithm was later implemented to improve uniformity of the beamlet array (discussed later in section 14.4).

\subsection{COMPARISON BETWEEN MULTIFOCAL GENERATION APPROACHES.}

Implementation of holographic beamlets permits individual spot flexibility with a significant improvement in uniformity in comparison with the Huygens-Fresnel approach. The phase projection of the GS algorithm occupies the full hologram space of the SLM, such that only one adaptive correction becomes necessary to compensate for optical aberrations in the optical train. The holographic projection example in Figure 28 and Figure 29 was performed using a Holoeye Pluto NIR2 SLM, which demonstrates significant phase distortion. However, once the SLM was replaced with a 16-bit Boulder HSPDM512 SLM the system aberrations were significantly reduced.

Phase patterns projected onto an SLM (Huygens-Fresnel and GS algorithm) permit individual manipulation of each focal spot, exhibiting higher flexibility capabilities than the microlens array.

The SLM generates Fraunhofer diffraction, which results in significant loss. Although the microlens array is more light efficient, SLM based methods permit appropriate beamlet alignment on the detector, which for emission collection is exceedingly more efficient (Section 12.2.1).

In the Fresnel lenses approach the hologram space corresponding to the SLM's microdisplay is divided into equally spaced regions for which each segment projects a Fresnel lens pattern. The incident light on each region undergoes a severe phase distortion, creating an interference pattern in the intermediate focal plane (Colyer 
et al., 2011). The Fresnel approach required adaptive correction for each region. In addition, each focal volume produced variable intensity due to the illumination profile of the laser. The GS algorithm produces a non-segmented pattern, generating the target intensity in the Fourier plane of the SLM. Projected in Hologram plane, the non-intuitive phase mask generated through iterations, demonstrates greater manipulation in addition to increased uniformity and beamlet numbers. The possibility of manipulating the phase hologram to produce a large variety of patterns permitted adequate characterization and development of various aspects of the system such as the optical crosstalk, optical ROI and successful alignment (Section 12.2.1 \& 14.5). The significant benefits of this approach over the microlens and the Huygens-Fresnel approach made the GS algorithm the most viable multifocal excitation method for the proposed application.

The beamlets projected within a sample undergo different refractive index mismatches, as the samples are morphologically different. Despite the disadvantages of using the Fresnel approach, it would be significantly simpler to provide a beamlet by beamlet correction. However for small excitation areas at the surface, the effect is minimal and significantly outweighed by the improvement in uniformity.

Lower NA objectives result in less errors between the calculated and target intensities for 3D holographic projections (Whyte and Courtial, 2005). However due to the quadratic intensity dependency in TPM a high NA is justified to produce tight focal volumes (Diaspro et al., 2006). 


\section{MULTIFOCAL MULTIPHOTON FLIM SYSTEM}

The following sections (Sections 11.1-11.5) are concerned with describing the final imaging system in terms of the optical (Section 11.1, 11.2), electrical (Section 11.4) and detector components (Section 11.5). The apparatus described was used to produce the results in Section 14.

The apparatus development in Sections 11.1-11.5 is described as two distinct stages characterized by the optical arrangement in Section 12. Subcategories of section 12 provide the description of the two stages separately. The first subcategory describes the non-descanned stage (Section 12.1), in which the galvanometer scan amplitude was calibrated along with software development and the first multifocal multiphoton image was acquired via stage scanning. Section 12.2 describes the development via descanned optical arrangement and beamlet optimization results.

\subsection{OPTICAL SYSTEM ARRANGEMENT}

The MegaFLI optical configuration over the course of the project was setup in two different manners. The non-descanned setup (Figure 32) served initially to establish the basic beamlet parameters; and the descanned setup (Figure 30) to make use of the galvanometer scanner to improve speed. This section is concerned with describing the final imaging system (descanned detection) in terms of the optical, electrical and detector components.

In all operational configurations using the Megaframe camera, a SLM is used to project the hologram required to generate beamlets via the GS algorithm.

As shown in Figure 30, laser light from the ultrafast Ti: Sapphire laser system in transverse mode $T E M_{00}$ [Coherent Chameleon Vision II] is expanded (x6) to overfill a Boulder HSPDM512 SLM. The appropriate phase pattern is calculated and projected onto the device's microdisplay. The pattern is sequentially relayed through a set of $x$ and $y$ galvanometer scanners onto the back aperture of the imaging objective. Sample fluorescence generated from the focused beamlet array is then collected and re-directed via a dichroic mirror onto the back aperture of an x10 0.3NA air objective [Nikon Instruments Inc., Japan] and focused onto a 
Megaframe SPAD array, which is mounted on a 3 axis piezo [MT-Z8 xyz stage Thorlabs Inc.] to aid the alignment of the beamlets onto the active area. Finer displacements are provided by an alignment procedure (Section 12.2.1).

Variable tuning range offered by the Ti: Sapphire permits the excitation of a large number of fluorophores. Power control via a half wave plate (HWP) and a polarizing beam splitter cube permits $6 \mathrm{~mW}$ per beamlet over the variable output power across the tuning range.

In the optical schematics shown (Figure 30, Figure 32, Figure 35) red and green lines represent the excitation and emission, respectively. An array of beamlets is generated in the image plane represented by a dashed line; relay optics project the pattern to the back of the objective. Fraunhofer diffraction generated via the SLM (not represented) is filtered via an aperture in the image plane.

\subsection{OPTICAL DESIGN RATIONALE}

In order to accurately design the system it is paramount to ensure that the fluorescence generated via the excitation in the sample plane can be accurately matched in the detector plane, while filling the field of view (FOV). In order to construct the optical system, the key design task is matching the fluorescence generated to the detection array. For this reason the size of the beam patterns in the microscope (focal and detection planes) will be discussed before considering how this pattern is generated at the SLM.

As established, the $32 \times 32$ SPAD array has a pitch between detector aperture centres of $50 \mu \mathrm{m}$; with overall dimensions of $1.65 \times 1.65 \mathrm{~mm}$. Therefore, it is necessary to define a separation factor, $\Omega$, of the beamlets produced in the $N \times N$ beam array, to assure correct reimaging of sample fluorescence onto the detection plane.

$$
\Omega=\frac{F O V}{N+1}
$$


In the detection plane $\Omega_{\text {detc }}$ (where the subscript defines the re-imaged plane of the beam array) is a minimum common multiple of $50 \mu \mathrm{m}$, as $N=32$ and the FOV is approximately $1.65 \mathrm{~mm}$.

Considering $\Omega$ for the sample plane, it is necessary to account for FOV of the imaging objective. The Plan Fluor x40 1.3NA has been shown to be a very useful objective for multiphoton imaging of single, attached cells using an inverted microscope and standard coverslips, due to high NIR transmission, high-collection efficiency and FOV of approximately $400 \mu \mathrm{m}$. Assuming full illumination of the pupil, the beamlet pitch in the sample plane $\left(\Omega_{\text {sample }}\right)$ is of $12.12 \mu \mathrm{m}$, which in a practical system can be rounded up to $12.5 \mu \mathrm{m}$. Therefore, to ensure correct reimaging of beamlets onto the detector:

$$
M \Omega_{\text {sample }}=\Omega_{\text {detc }} \quad 44
$$

Hence $M=4$ magnification between the imaging objective and the detection objective to approximately fill the field of view. As the tube lens $\left(L_{8}\right)$ has focal length $=200 \mathrm{~mm}$, the magnification of the image $\left(I_{1}\right)$ in the plane produced between the scan $\left(L_{7}=100 \mathrm{~mm}\right)$ and tube lens is defined by the magnification of the objective (x40). Consequently the FOV of $I_{1}$ is $16.5 \mathrm{~mm}$ with $\Omega_{\text {excitation/detection }}=500 \mu \mathrm{m}$. Therefore $\times 10$ demagnification of $I_{1}$ produces $1.65 \mathrm{~mm}$ FOV with a $50 \mu \mathrm{m}$ pitch, matching the requirements for a successful relay onto the detection plane $\left(I_{d}\right)$.

However, imaging using the 2-axis galvanometer scanner and subsequent reprojection (Figure 30) conveys extra considerations. The scanning system developed in the Ameer-Beg group for large aperture imaging in TPM (predominantly this architecture was adopted in implementations of FLIM systems where pupil engineering was potentially to be used) was implemented in this scheme for purely practical reasons. Principally, the scanner system has a $6 \mathrm{~mm}$ aperture, enabling large beams to be used with low magnification between final scan mirror and the objective. The system consists of a pair of galvanometer scanners re-imaged via a $4 f$ arrangement since there are four separating distances of length $f$ between the input and output plane (Goodman, 1996). A second multifocal image plane $\left(I_{2}\right)$ is 
generated between the scan lens $L_{5 / 6}\left(f\left(L_{5,6}\right)=50 \mathrm{~mm}\right)$, with $F O V\left(I_{2}\right)=$ $8.25 \mathrm{~mm}$ and $\Omega\left(I_{2}\right)=250 \mu \mathrm{m}$ consequence of the 0.5 magnification between $L_{6}$ and $L_{7}$. The dichroic filter separates the descanned fluorescence from the excitation and undergoes $\times 2$ demagnification using the telescope $L_{9} / L_{10}$ (100 and $200 \mathrm{~mm}$ respectively) generating a third intermediate image plane $\left(I_{3}\right)$ providing $\mathrm{FOV}\left(I_{3}\right)=16.5 \mathrm{~mm}$ and $\Omega\left(I_{3}\right)=500 \mu \mathrm{m}$. The fluorescence is finally focused onto the Megaframe SPAD array $\left(I_{4}\right)$ using an x10 0.3 NA air objective, resulting in a $\operatorname{FOV}\left(I_{4}\right)=1.65 \mathrm{~mm}$ and $\Omega\left(I_{4}\right)=50 \mu \mathrm{m}$ characteristic of the multidetection design.

Finally, there is the matter of matching the excitation array to the microscope. The excitation undergoes an $x 6$ expansion due to the $L_{1} / L_{2}$ telescope (40 $\mathrm{mm}$ and 250 $\mathrm{mm}$ respectively) to overfill the SLM at an $18.5^{\circ}$ angle as to produce a perpendicular first diffractive order. Matching the excitation to the system is a matter of ensuring that the pitch of the excitation $\left(\Omega_{\text {exc }}\right)$ is equal to the pitch of the required emission $\left(\Omega_{e m}\right)$ at the second image plane $I_{2}$. Such that:

$$
\Omega\left(I_{2}\right)=\Omega_{\text {exc }}\left(I_{2}\right)=\Omega_{\text {em }}\left(I_{2}\right) \quad 45
$$

The SLM positioned in the Fourier plane of a $4 f$ system, for which $L_{3}\left(f_{3}=\right.$ $500 \mathrm{~mm}$ ) produces the multifocal excitation array $\left(I_{5}\right)$ located in the Fourier plane of the SLM. An aperture was placed in the Fourier plane of the $\operatorname{SLM}\left(I_{5}\right)$, between lenses $\left(L_{3} / L_{4}\right)$ to filter out the first order pattern from the zero order light (Spalding et al., 2008). The magnification of $0.6\left(f\left(L_{4}\right)=300 \mathrm{~mm}\right)$ assures that the beamlets produced with $150 \mu \mathrm{m}$ pitch at $I_{5}$ have a $250 \mu \mathrm{m}$ pitch at $I_{2}$. As $I_{5}$ is the result of holographic projection, the method provides sufficient adaptability to individually generate flexible pitches $\left(\Omega_{\text {ex }}\right)$ (Grier, 2003), important for the alignment procedure onto the SPAD's. The optical system can be designed to accommodate a commercially available microlens array (such as the Thorlabs array discussed in section 10.1). However as shown in section $10 \& 12.2 .1$, other issues must be considered when using a refractive lens array. 


\subsection{OPTICAL LAYOUT: DESCANNED DETECTION}

In the descanned configuration, beamlets generated using a Boulder HSPDM512 were focused using x40 1.3 NA oil objective [Nikon Instruments Inc., Japan]. Beamlets scan the sample via the galvanometer scanner. The hologram displayed on the SLM relayed onto the back focal plane of the objective slightly under fills the back aperture effectively reducing NA to 1.2 (Section 3.1 and Figure 30).

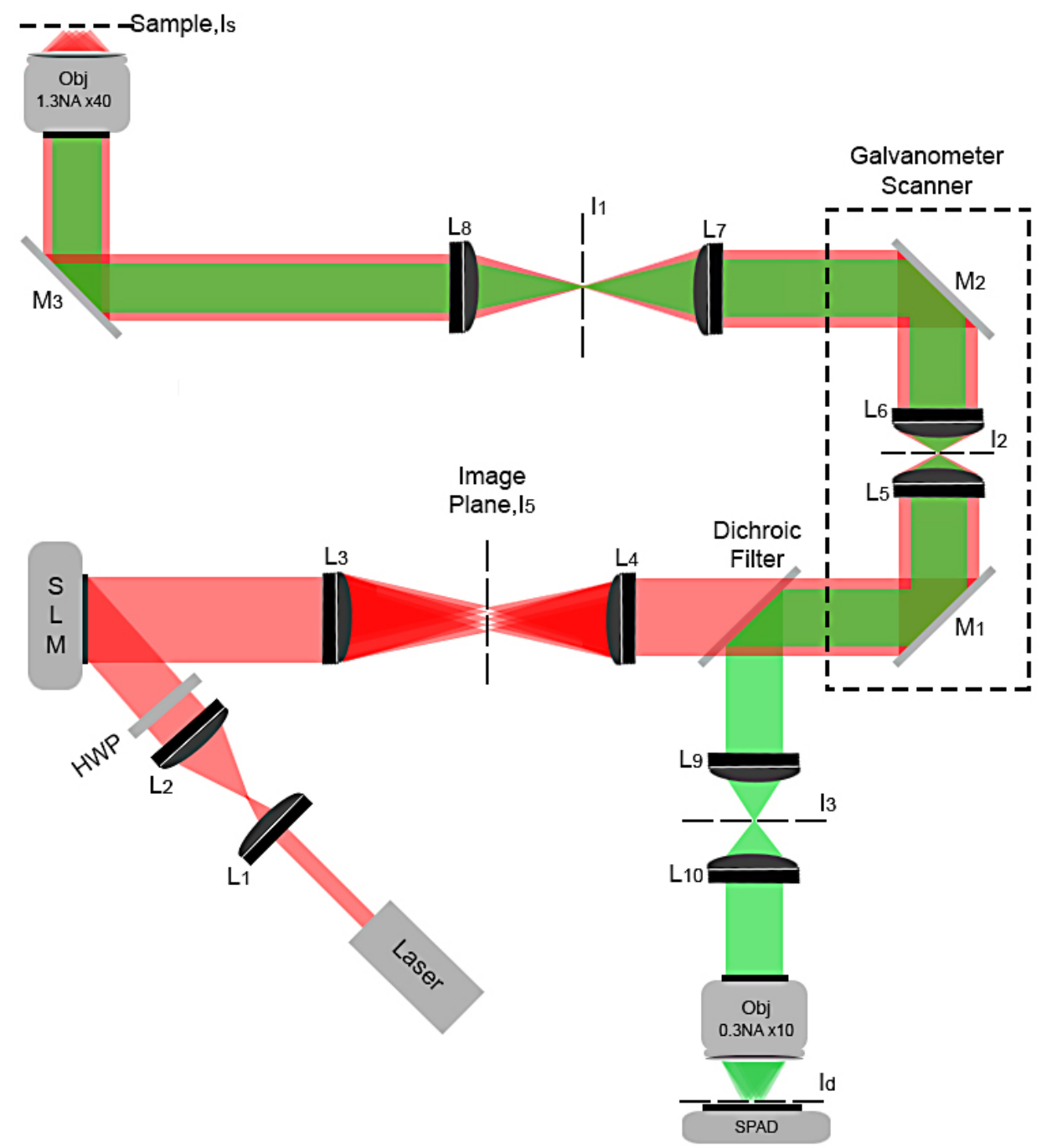

Figure 30: MegaFLI schematic in the descanned configuration. OBJ: objective lens, HWP: half-wave plate. The $200 \mathrm{~mm}$ tube lens is housed in a Nikon Ti-E microscope body and the illumination source is a Coherent Chameleon Vision II. Image planes represented by $I_{X}$ are multifocal excitation (red)/fluorescence (green) planes consequence of the GS algorithm hologram projection on the SLM located in the Fourier plane of $L_{3}$ (lenses and mirrors denoted $L_{x}$ and $M_{x}$ ) (Section 11.2). 


\subsection{ELECTRONIC CONTROL}

The Ti: Sapphire laser is connected to the host PC via a standard RS-232 connection, permitting wavelength change and shutter control. Due to response delay, the laser shutter is left open and a $12.5 \mathrm{~mm}$ diameter external optical shutter [Thorlabs SH05] is controlled via a standard multifunction NI-DAQ [National Instruments USB 6259]. The DAQ analogue outputs drive the scan mirrors [Cambridge Tech.] via software generated voltage waveforms. A TTL trigger generated by the DAQ board provides synchronization between the mirror scan and the detector, ensuring that histograms are calculated for the pixel of interest over a user-defined period. A beam pickoff reflects $\sim 3 \%$ of the light onto an optical constant fraction discriminator [B\&H, OCF-400] which generates laser pulse synchronization with the Megaframe. The detector was mounted on a motorized 3-axis stepper xyz stage [Thorlabs Inc. - MT3-Z8] to aid alignment (Section 12.2.2). The SLM is connected to the host PC via a DVI and a standard RS232 connection. The operational response time of the LCD layer after switching patterns requires a stabilization period of 5 frames, negligible for our application.

The Nikon Ti-E inverted microscope body is connected via USB and controlled using a LabVIEW interface (developed by Dr. Daniel Matthews). Integration permits automatic filter-cube rotation for widefield excitation, axial displacement for 3dimensional acquisitions along with lateral movement for sample movement and stage scanning. Laser illumination enters in the left port and the right port has a CCD camera [Orca, Hamamatsu] for widefield imaging.

\subsection{MEGAFRAME READOUT}

The Megaframe camera array is split into two 32x16 sub-arrays of pixels (Figure 31). One bit of data is transferred per pixel clock. As each TDC is 10bits, a total of 10 pixel clocks are needed to transfer an individual row from both sub-arrays. Each 32element row of data is de-serialized and read out in sequence. At full data rate, $500 \mathrm{kHz}$, where each frame composed of 1024 pixels (32×32), top and bottom data 
channels generate output data at $\sim 5 \mathrm{Gbps}$. As $5 \mathrm{Gbps}$ is not supported, the FPGA firmware compares the 640 deserialized data lines and transfers only data acquired within a user selected 64-pixel ROI. The TDC timestamps for selected pixels are placed in the first-in-first-out (FIFO) buffer. Each timestamp is 16 bits: 10 bits of time data, 5 bits of addressing of 64 pixels and one spare bit. The FIFO buffer is read via USB2 link to the host PC allowing for up to $16 \mathrm{Mbps}$ transfer rate (Poland et al, 2014 Biomedical Optics Express, Submitted).

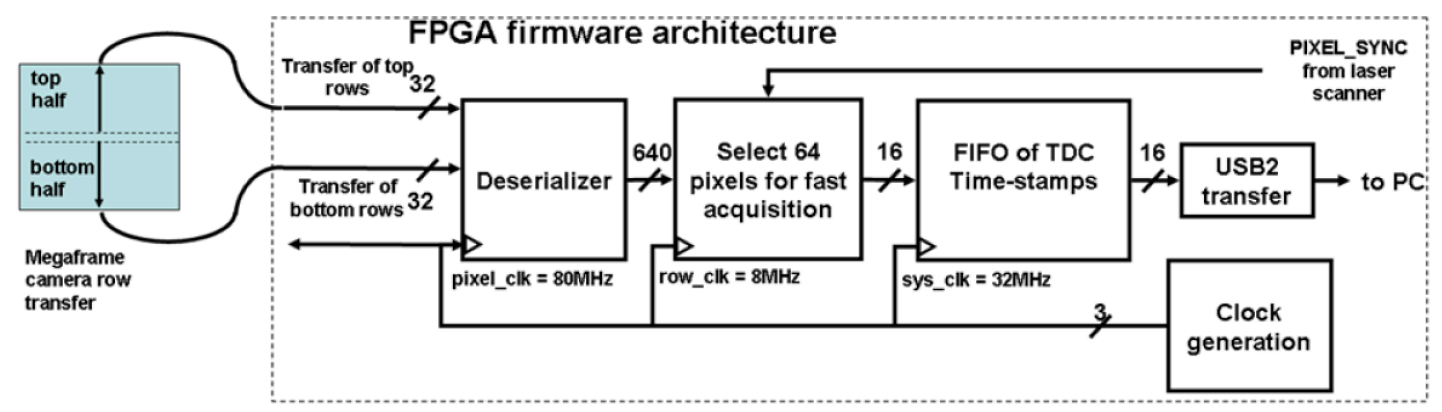

Figure 31: Megaframe firmware architecture schematic. Megaframe camera array is split into two $32 \times 16$ sub-arrays of pixels. The data is de-serialized, read out in sequence and data acquired within a user selected 64-pixel ROI is transferred. The TDC timestamps are placed in the FIFO buffer, which is transferred via USB2 to the host PC

\section{MULTIFOCAL MULTIPHOTON FLIM DEVELOPMENTAL STAGES}

The system development shall be characterized by two distinct stages, depending on the optical layout. The first developmental stage is the nondescanned detection. This served to calibrate the scan size, initial software integration and development and provide the first image using the multifocal excitation with the SPAD array architecture via stage scanning. The second developmental stage is the descanned detection. The descanned detection is the final system arrangement used for high-speed FLIM imaging (described in Sections 11.1-11.5). Beamlet optimization such as multifocal alignment (Section 12.2.1) and GS algorithm uniformity improvement (Section 12.2.3) was performed in the descanned detection. 


\subsection{MULTIFOCAL MULTIPHOTON FLIM: NON-DESCANNED DETECTION}

(DEVELOPMENTAL STAGE I)

In the non-descanned configuration, beamlets generated using a Holoeye Pluto NIR2 (Osten et al., 2007) are relayed and focused using x20 0.5NA air objective [Nikon Instruments Inc., Japan] onto the sample. Stationary beamlets excite the sample, which is moved in the $x$ and $y$ plane in a raster pattern using the Nikon Ti-E stage. The optical rationale follows the description given in Section 11.1.

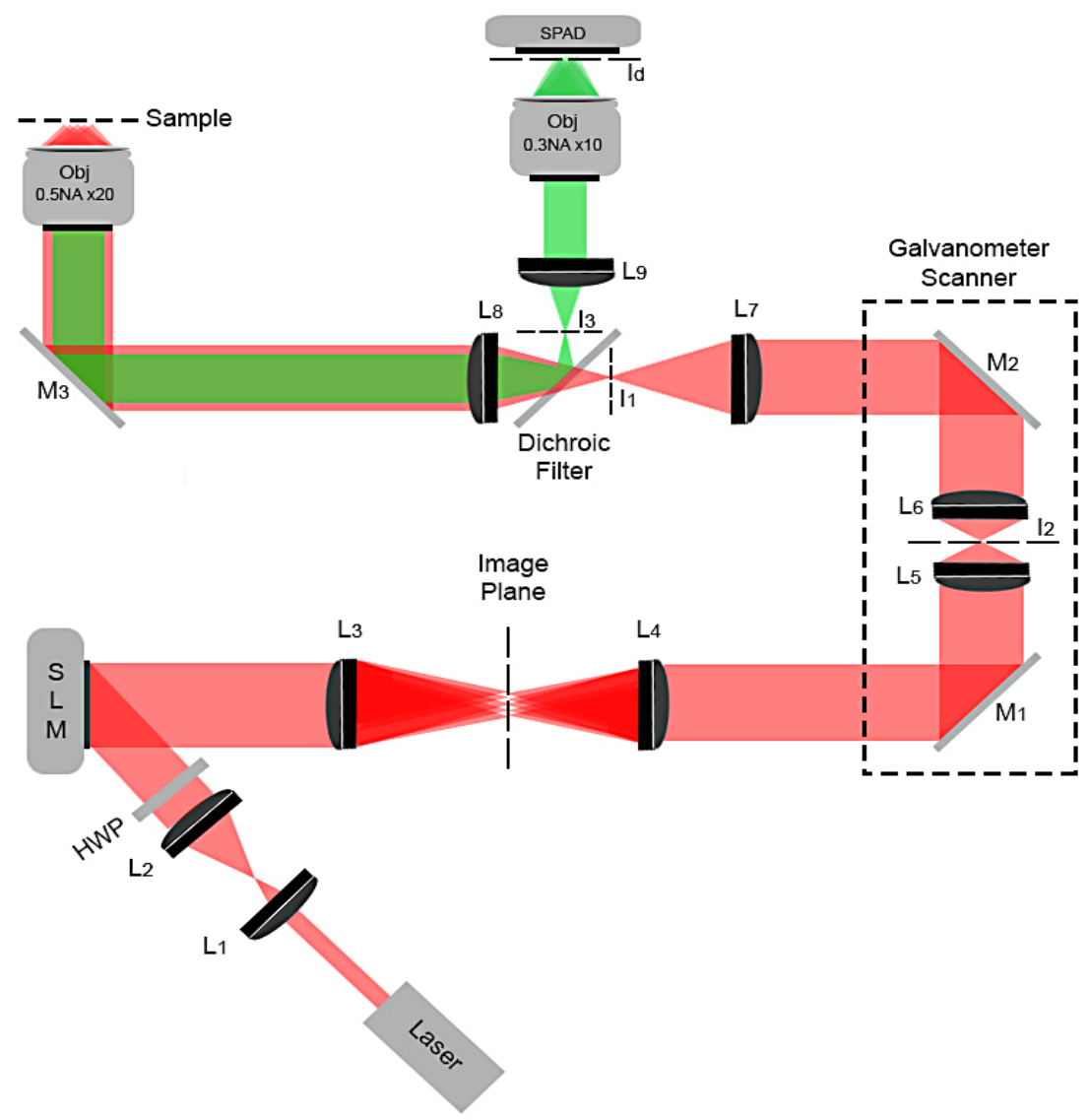

Figure 32: MegaFLI schematic. OBJ: objective lens, HWP: half-wave plate. The $200 \mathrm{~mm}$ tube lens is housed in a Nikon Ti-E microscope body and the illumination source is a Coherent Chameleon Vision II. Image planes represented by $I_{X}$ are multifocal excitation (red)/fluorescence (green) planes consequence of the GS algorithm hologram projection on the SLM located in the Fourier plane of $L_{3}$ (lenses and mirrors denoted as $L_{x}$ and $M_{x}$ ). Relay optics in the excitation between $L_{4}$ and $M_{1}$ have been omitted. Optical rationale detailed in Section 11.2. 
The first step taken to test the viability of the approach was to place the SPAD detector in the non-descanned position. Once the beamlets were aligned, the sample was moved in the $x$ and $y$ directions in a raster pattern in order to build an image, using the microscope stage. With the SPAD in TCSPC mode, enabling 48 full frames simultaneously, a total acquisition of 4800 frames for each pixel acquisition (maximum of $50 \mathrm{fps}$ ). The system was set up to process $50 \times 50$ data points for $5 \times 5$ detectors producing $250 \times 250$ pixel images. Full frame mode was the first firmware implementation available for the project and essentially transferred $32 \times 32$ detector time-stamps. A Convallaria majalis sample (Leica) stained with Safranin Red and Fast Green (Figure 33) acquired over a total of 40 minutes, shows a detector-bydetector intensity normalised image of the acquired intensity and lifetime contrast. It is possible to verify line artefacts within the data. This results from a discontinuity /overlapping acquisition in the data set. As the each beamlet independently scans a square section of the image, the line artefacts need to be corrected via appropriate image stitching (Section 13.1).
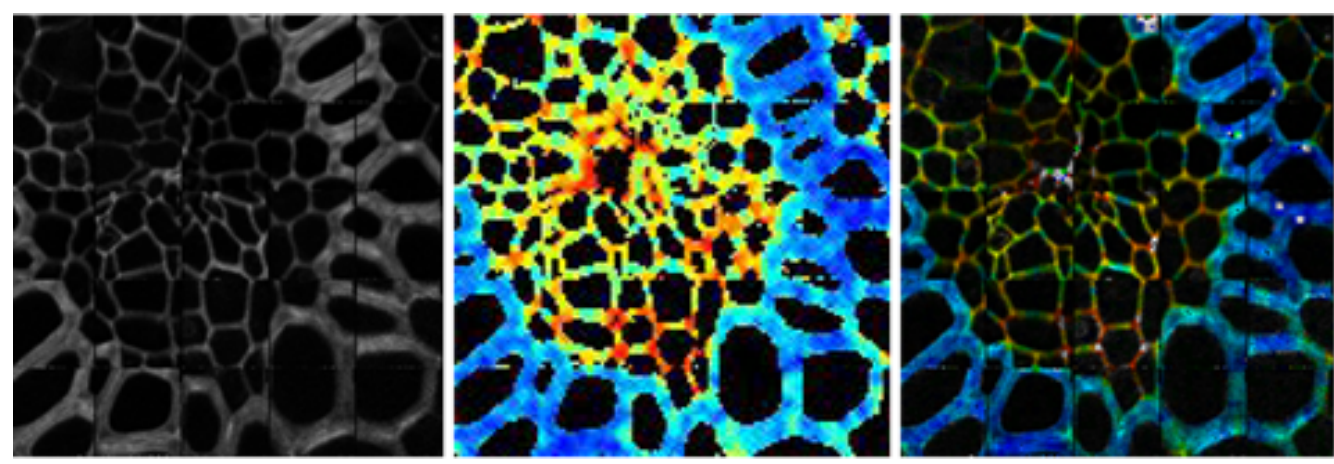

2 ns

Figure 33: Multifocal multiphoton image of fixed Convallaria majaralis sample (Leica) in the non-descanned detection path. A $125 \times 125 \mu \mathrm{m}$ area was acquired with a 25 square beamlet array with $800 \mathrm{~nm}$ excitation wavelength and emission filter with $40 \mathrm{~nm}$ bandwidth centred at $520 \mathrm{~nm}$. Individually normalized acquired intensity (left) is merged with the lifetime (middle) to provide a combined image (right) confirming the precise overlap of the lifetime and intensity positions.

Initially the system was configured in the non-descanned detection path as an intermediate step to establish adequate parameters such as scan size, initial software integration and optical development of beamlet patterns. Successful 
transition to descanned detection requires adequate scan calibration. Calibration of the galvanometer scan size was determined by exciting a green fluorescent block with a $3 \times 3$ beamlet array. Using the galvanometer scanner, the fluorescence generated by raster scanning the beams, is re-imaged onto the non-descanned detector, therefore scanning the surface of the detector array while performing an intensity image acquisition (Figure 34). As the detector array has $50 \mu \mathrm{m}$ separation between detector apertures, the galvanometer scanner voltages can be calibrated by measuring the number of pixels (steps) between beamlets striking the detector aperture. Calibration scans showed a $1 \mathrm{mV}$ step size was equivalent to $0.76 \mu \mathrm{m}$ in the detector plane (or $1.3 \mathrm{mV}$ corresponding to $1 \mu \mathrm{m}$ ). We are therefore able to calibrate the scan amplitude for each beamlet.

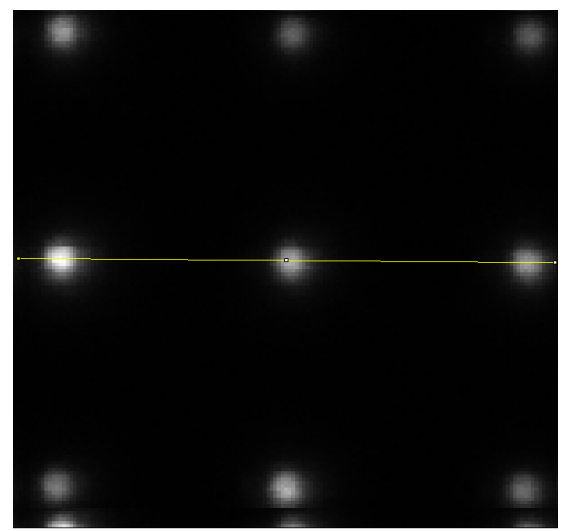

\section{Line profile}

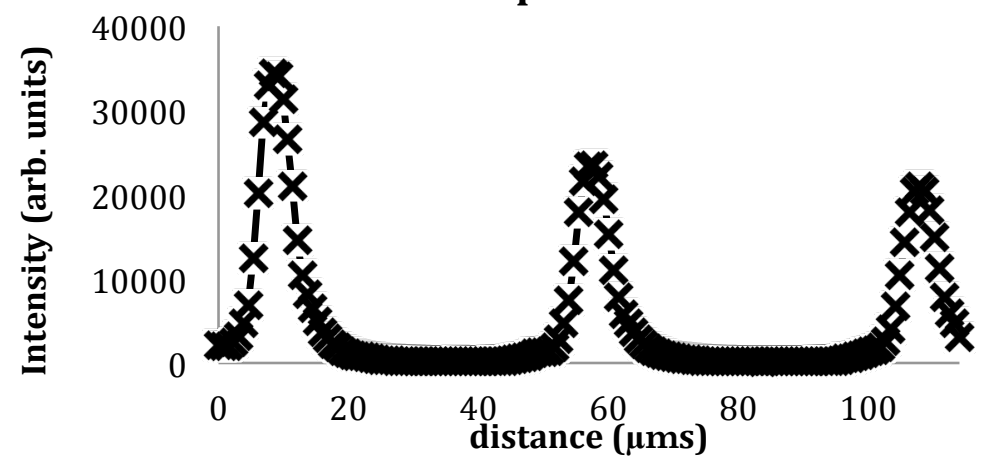

Figure 34: Intensity (150x150 pixels) response and line profile of a $3 \times 3$ generated fluorescence scanned over an individual SPAD array. 


\subsection{MULTIFOCAL MULTIPHOTON FLIM: DESCANNED DETECTION (DEVELOPMENTAL STAGE II)}

In the descanned configuration, beamlets generated using a Boulder HSPDM512 were focused using x40 1.3 NA oil objective [Nikon Instruments Inc., Japan]. Beamlets scan the sample via the galvanometer scanner. The hologram displayed on the SLM relayed onto the back focal plane of the objective slightly under fills the back aperture effectively reducing NA to 1.2 (description in Section 3.1 and Figure 30).

\subsubsection{BEAMLET OPTIMIZATION}

The following section describes optimization procedures for multifocal alignment (section 12.2.2), GS algorithm uniformity improvement (section 12.2.3).

\subsubsection{POSITION OPTIMIZATION}

In order to align the fluorescence beamlets generated onto the small active areas of detector array (50 $\mu \mathrm{m}$ pitch), it was necessary to develop an adequate alignment procedure.

As an initial approach, the surface of the SPAD array was set up such that it could be viewed in reflection. A CMOS camera reimaging the surface of the SPAD (Figure 35) served to visually match the fluorescent displacement with the detector position. The manual beamlet alignment setup required a beam splitter cube and a camera, placed to reflect the surface of the SPAD. Achieving the correct $z$ plane and approximate $x y$ consisted of simply adjusting the motorized 3-axis stepper control (MT3-Z8 xyz stage - Thorlabs Inc.) on which the detector was mounted. To facilitate rotational offset, the GS algorithm was manipulated to provide an extra degree of freedom via rotation of the projected points around a common axis. Finally each beamlet was individually modified in $x y$ to match the corresponding detector. This method was shown to be a straightforward approach to set up the system. Despite the simplicity of a direct alignment, it also reduced the fluorescence by $50 \%$ due to 
the presence of a beam splitter in the optical train. It was also time consuming and sensitive to slight misalignments or minor modifications of the system.

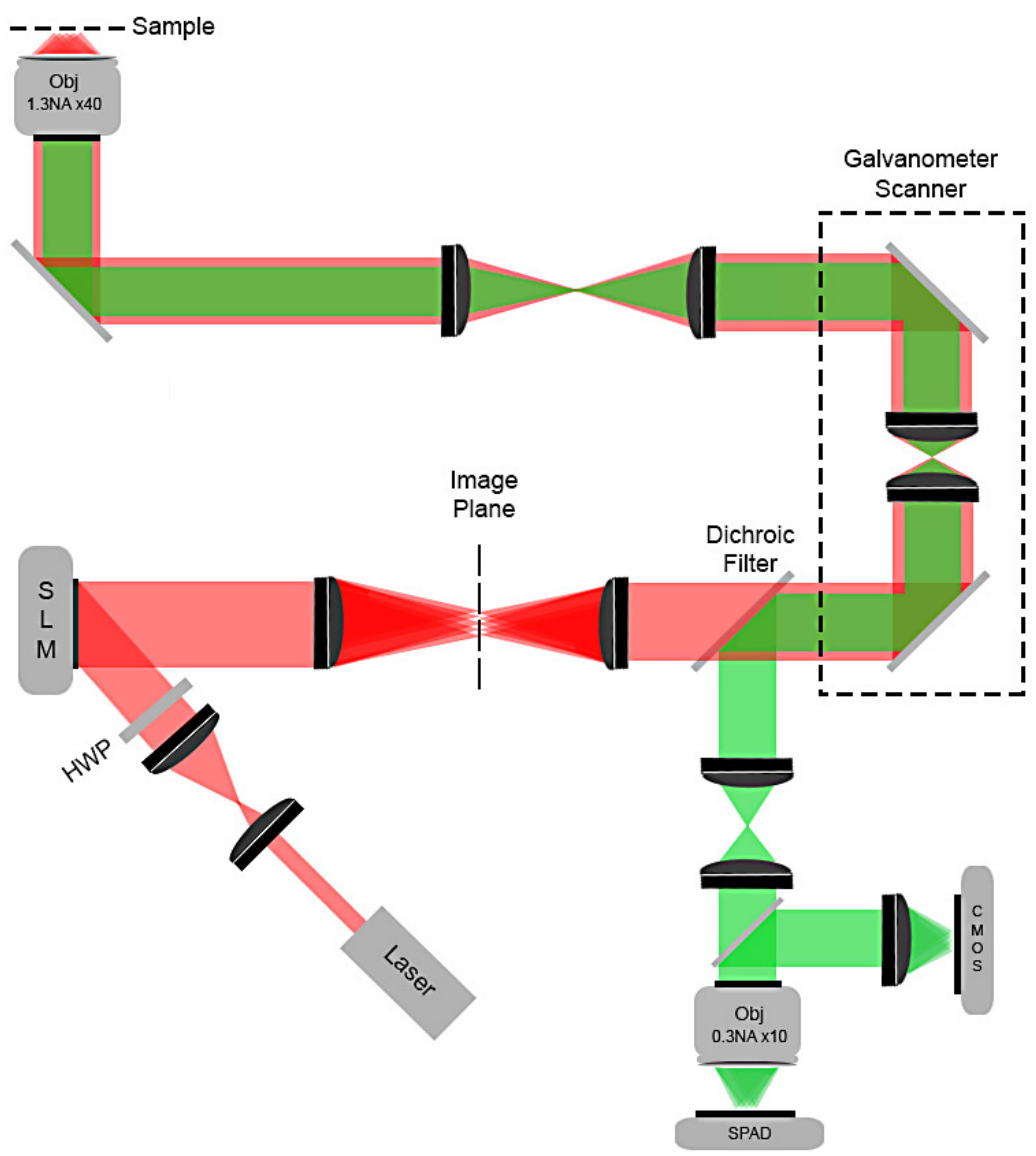

Figure 35: Multifocal manual alignment setup. OBJ: objective lens, HWP: half-wave plate. The $200 \mathrm{~mm}$ tube lens is housed in a Nikon Ti-E microscope body and the illumination source is a Coherent Chameleon Vision II. Multifocal multiphoton laser light is represented in red and the fluorescence is in green. The CMOS camera is configured in reflectance to monitor the illuminating surface of the SPAD. Reflected imaging performed by a beam splitter cube and focused by a $200 \mathrm{~mm}$ lens. Optical layout detailed in section 3.1.

In order to counteract the disadvantages of manual alignment, an automatic alignment procedure was developed utilizing the Megaframe device for optical feedback. Automatic beamlet alignment consisted of stepping the excitation beamlets focal positions in $X Y$ in the sample by altering the hologram target 
intensities. The GS algorithm was modified to provide a point-by-point displacement of the beamlets, therefore generating a 'scanless' (no mechanically moving parts) (Nikolenko et al., 2008) two-photon excitation of a green fluorescent block (Figure 36). Multifocal patterns sequentially producing a raster scan, results in a 2D displacement of the emission across the SPAD's surface. Iteratively registering the intensities permits mapping of the detectors. The detectors running synchronized with a scanning procedure produces a final intensity image in which the maximum intensities correspond to the position of the detectors. Consequently the user-friendly automatic alignment procedure provided a faster, more precise approach of the beamlet alignment with the added benefit of removing the beam splitter cube which introduced losses, if integrated, or additional tip-tilt once removed; requiring additional alignment. 

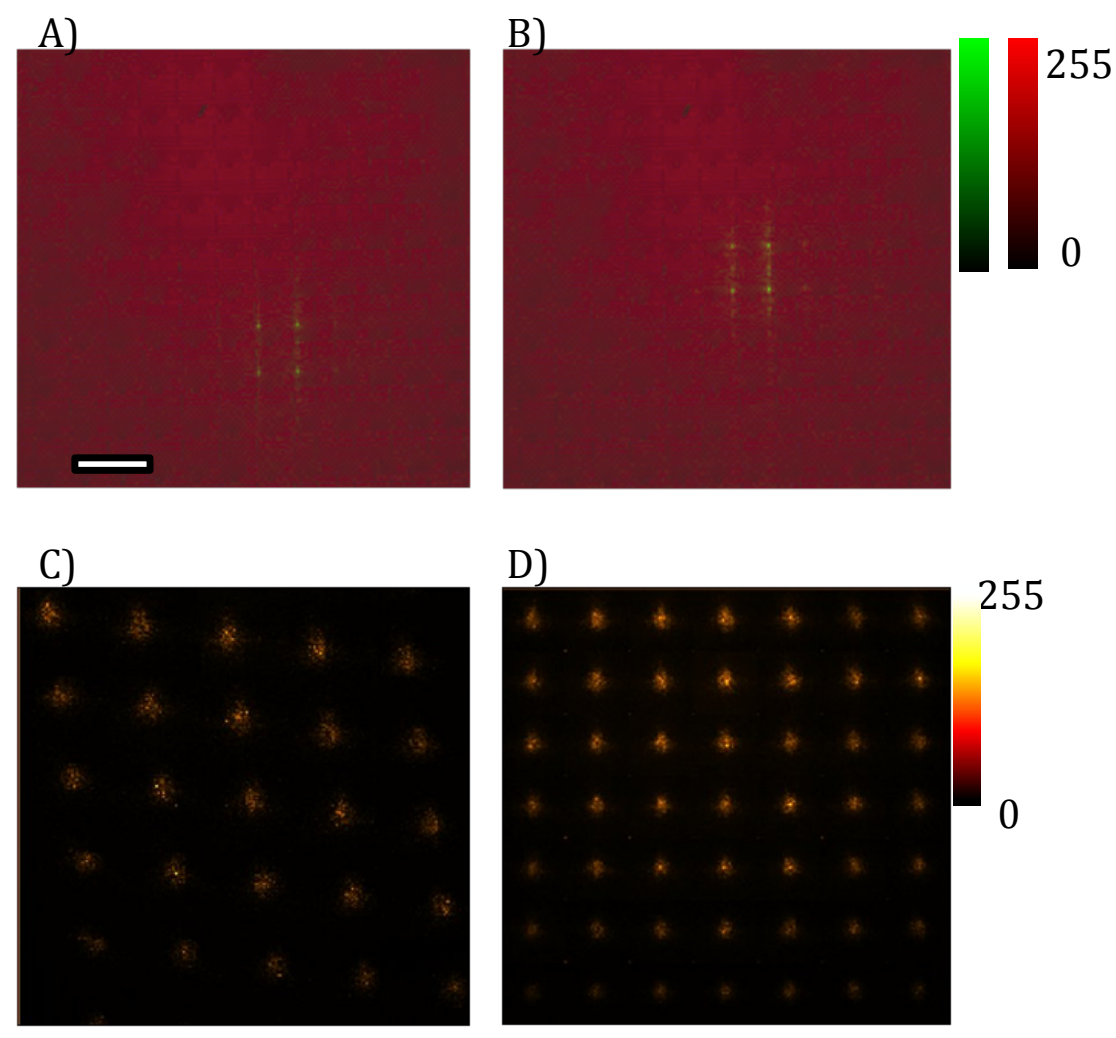

Figure 36: Comparison of manual (A/B) with automatic alignment procedure (C/D). Manual alignment steps illustrated: Original reflection detected (A) (red and green represent SPAD and fluorescence reflection), rotational correction and minor translational correction and full alignment onto less noisy SPAD region (B). Automatic alignment procedure demonstrated: Original intensities detected (C), centre-of-mass calculation to correct for GS spots positining provides realignment on the detectors (D). Automatic alignement provides a more light efficient (due to abcence of beam splitter) and more accurate alignment procedure.

\subsubsection{WEIGHTED GERCHBERG-SAXTON ALGORITHM}

Regardless of the much improved uniformity offered by the GS algorithm in comparison to the Huygens-Fresnel generation, slight heterogeneity remains after minimisation, providing an approximate 60\% uniformity (Spalding et al., 2008). Hologram uniformity, $U$, can be quantitatively defined as:

$$
U=1-\frac{I_{\max }-I_{\min }}{I_{\max }+I_{\min }}
$$


In which $I_{\max }$ and $I_{\min }$ is the maximum and minimum intensities registered in the target plane. Commonly applied in holographic optical tweezing, equation 46 is used to evaluate trap efficiency. The algorithm's increase in uniformity results in improved trap efficiency (Spalding et al., 2008).

In order to improve homogeneity of the beamlets within our imaging system, where intensity differences between beams will be increased due to the $1^{2}$ weighting of the two-photon fluorescence signal, a weighted GS algorithm was implemented. Higher uniformity of the intensity distribution is obtained by introducing an additional degree of freedom, $w_{m}$. The initial case assumes that all initial weights are set to $1\left(w_{n}^{0}=1\right)$. Illumination variations are minimized using a weighted sum $\sum_{n} w_{n}\left|u_{n}^{T}\right|$ with the constraint that all $\left|u_{n}^{T}\right|$ are equal. In this method, the GS algorithm weighs the current $w_{n}^{k}$ calculated from those of the previous iteration, $w_{n}^{k-1}$ and then proceeds onto calculating the GS algorithm with a weighted coefficient (Di Leonardo et al., 2007; Spalding et al., 2008). The weights are calculated according to:

$$
w_{n}^{k}=w_{n}^{(k-1)} \frac{\left\langle\left|u_{n}^{k-1}\right|\right\rangle}{\left|u_{n}^{k-1}\right|}
$$

The weighted GS algorithm has been broadly applied in holographic optical tweezing to regularly produce optical traps. The weighted GS algorithm has been shown to produce near ideal performance with uniformities of approximately $99 \%$ (Di Leonardo et al., 2007) at a cost of computation time.

In order to produce a flat illumination and detection profile for the beamlets, a number of additional modifications to the beamlet illumination scheme were considered. A postdoctoral researcher in the Ameer-Beg group ( $\mathrm{Dr}$ Simon Poland) implemented such a method to improve the homogeneity of the beamlets using a weighted GS algorithm. Effective optimization reduces variations across the excitation by multiplying the target intensity by a weighting coefficient $\left(w_{m}\right)$. Under the principle that $I_{T}$ corresponds to the target intensity, for a field, $u_{n}^{T}$ equal for all beamlets, an iterative optimization provides incremental approximations to an ideal weighting factor. 
Emission from stationary excitation foci from a green fluorescent block is imaged onto a section of the SPAD array producing lower dark counts. The detector produces a variable DCR across the pixels, influencing the flatting. The weighing coefficient improves detection uniformity across an array by influencing the excitation via $w_{m}$. The results shown (Figure 37) are a comparative chart demonstrating a $30 \%$ improvement in uniformity from a fluorescent block once compared to the standard GS algorithm. The WGS is used throughout the remaining of this chapter.

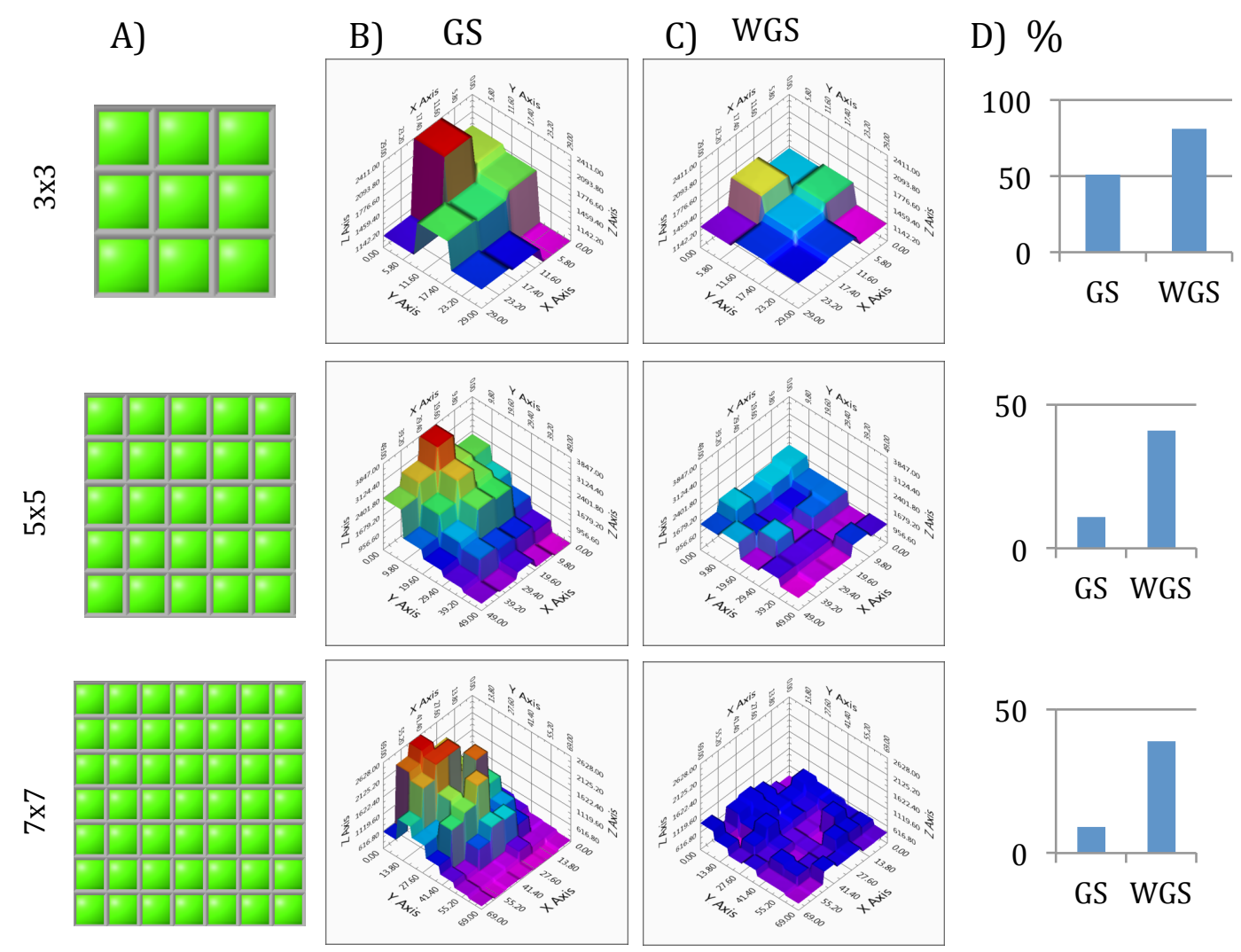

Figure 37: Uniformity comparison chart between GS and weighted GS algorithm for various beamlet arrays generated. Multifocal pattern projected in the column A. The fluorescent intensity of a standard array of beamlets produced (column B) shows increased uniformity (column C) once the WGS is implemented. Uniformity presented in accordance to Eq. 46 (column D). 
The uniformity in comparison to a beam splitter cube approach is expected to be lower. A single beam is passed repeatedly through a series of beam splitter cubes; therefore the variation in illumination is lower. This form of illumination produces equal foci in a simple arrangement as used in the TriM Scope (Kumar et al., 2007a). However manipulation of the foci in $2 D / 3 D$ is severally restricted. The microlens array shows a high number of beamlets produced in an increasingly simpler optical configuration, however as the beam splitter cube approach, adequate alignment serves technically challenging due to individual foci manipulation along with the increased sensitivity to aberrations demonstrated. Therefore due to the SPADs small active area manipulation of the focal volumes position becomes ever more important. The WGS algorithm employed is capable of providing sufficient manipulation along an adequate uniformity over the detector array (Figure 37 and Figure 44). 


\subsection{IMAGE STITCHING AND ALIGNMENT}

Fluorescence generated from each excitation beamlet is descanned and re-imaged onto an individual SPAD operating in TCSPC mode. For a single beam excitation, the focal spot scans a square region. In the multifocal case, each beam scans a subregion of the final image. The size of each individual region equals $12.5 \mu \mathrm{m}(32 \times 32$ data points). The final image $(256 \times 256$ pixels $)$ is a 'composite' of the multifocal/multi-detection combined scan regions $(32 \times 32$ data points from $8 \times 8$ detectors). In order to accurately couple the independent detector acquisitions, a 2-pixel overlap between detector regions permits image stitching and alignment (Figure 38). The boundary of each detector is oversampled, generating a false representation of the real values. Adequate stitching and realignment of the mismatched overlapping sections at the edge of each sub-image eliminates the duplicated values. The final image size is $240 \times 240$ pixels. Effective stitching and alignment software (created by Dr. Simon Poland) reduces positional variations introduced through oversampling across the detectors by replacing the overlapping pixel. Realignment compensates for the relative positional variations generated from the parallel-acquired detector segments with respect to each other. The software also eliminates line artefacts from the scanning process (Figure 38). 


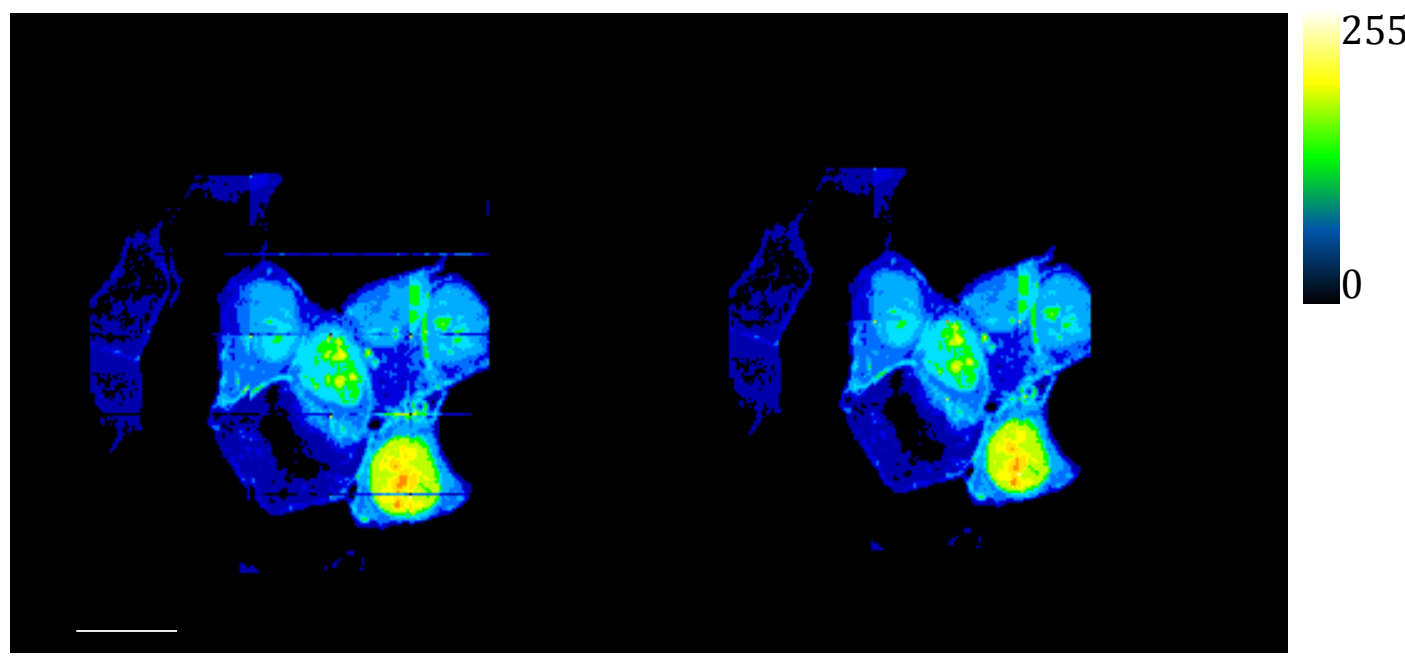

Figure 38: Image alignment and stitching. Original intensity image (left) shows

higher than background intensity lines on the border separating detectors consequence of a scanning artefact. Eliminating the duplicated values at the edge of each sub-image reduces image size to $240 \times 240$ pixels. The compensated image is shown on the right. Images have an added false colour LUT to aid visualization.

In order to appropriately analyse acquired data, it is necessary to first perform batch realignment of the images and then subsequently save as an ics format for lifetime analysis in TRI2. This realignment treatment is processed post-acquisition, but in future could be implemented "on-the-fly", improving data manipulation.

\subsection{TCSPC-FLIM IMAGE ANALYSIS}

In order to produce an appropriate estimation of the lifetime of the fluorophores it is necessary to fit the fluorescence lifetime transient accordingly. Non-interacting and interacting sub-populations have distinct fluorescent lifetimes. Fitting the data with a bi-exponential fluorescence decay model by iterative reconvolution while assuming the second exponential component relates to the interacting population, it is possible to determine the FRET efficiency and interacting fraction $\alpha_{2}$ (Barber et al., 2009; Barber et al., 2005).

$$
\left.I_{c}(t)=I_{0} \int_{-\infty}^{\infty} I_{\text {instr }}\left(t-t^{\prime}\right) \cdot\left(\alpha_{1} e^{-\left(\frac{t}{\tau_{1}}\right)}+\alpha_{2} e^{-\left(\frac{t}{\tau_{2}}\right)}\right)\right) d t^{\prime} \quad 48
$$


In which:

$$
\alpha_{1}+\alpha_{2}=1
$$

And $I_{\text {instr }}(t)$ is the instrumental response, $I_{0}$ is the peak intensity; $\alpha_{1}$ and $\alpha_{2}$ are the fractional proportions of the lifetimes, $\tau_{1}$ and $\tau_{2}$, respectively. The reduced goodness-of-fit parameter, $\chi^{2}$ is defined by:

$$
\chi^{2}=\frac{\sum_{k=1}^{n} \frac{\left[I\left(t_{k}\right)-I_{C}\left(t_{k}\right)\right]^{2}}{I\left(t_{k}\right)}}{n-p}
$$

Where $I\left(t_{k}\right)$ is the data and $I_{c}\left(t_{k}\right)$ the fit value at the $k^{t h}$ time point, $t_{k} ; \mathrm{n}$ is the number of time points and $p$ the number of variable fit parameters. Appropriate fitting produces a $\chi^{2} \sim 1$ with a random distribution of the weighted residuals around zero.

The acquired image is saved in a B\&H standard sdt file format. After stitching and alignment is performed, the image is ready for time-resolved image analysis, performed in TRI2. The TRI2 lifetime analysis software was developed in-house by Dr Paul Barber (King's College London and University of Oxford. http://users.ox.ac.uk/ raob0009/software.html), the package has considerable functionality and is capable of fitting to a large variety fitting models in addition to specialised methods such as Bayesian and Phasor analysis (Barber et al., 2009; Barber et al., 2005; Barber et al., 2008).

In the context of this thesis, data was analysed using either a mono or biexponential (as appropriate) using the Levenberg-Marquardt minimization procedure (Figure 39). Fitting produces $\chi^{2}$ error estimation (Figure 40 ) for each transient in addition to an appropriate lifetime image. Represented under an inverse rainbow look-up table throughout this thesis, short lifetime times appear red, for example demonstrating FRET activity. CMOS-SPAD fabrication process produces variable bin sizes between detectors such that slight differences in temporal resolution are observed and lead also to different IRFs (Krstajić et al., 2013); these slight variations have not been accounted for in the data presented 
here since characterisation of the detectors was not adequately performed for the data acquired. However, this correction is possible as long as the bin widths are known.

\section{Mono-exponential (Levenberg-Marquardt) GFP cells}

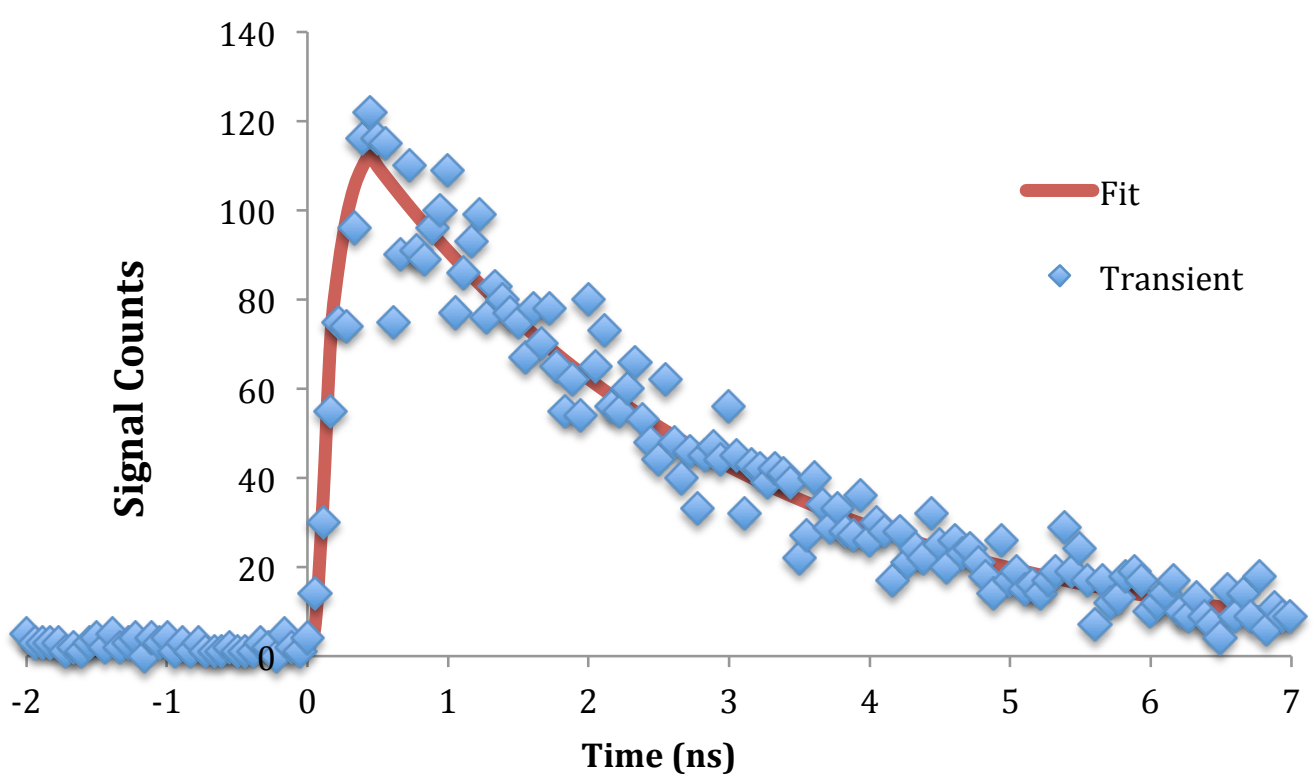

Figure 39: Typical transient produced by the MM-FLIM system. The LevenbergMarquardt mono-exponential fit is represented and red and the data points in blue.

\section{Residual Distribution}

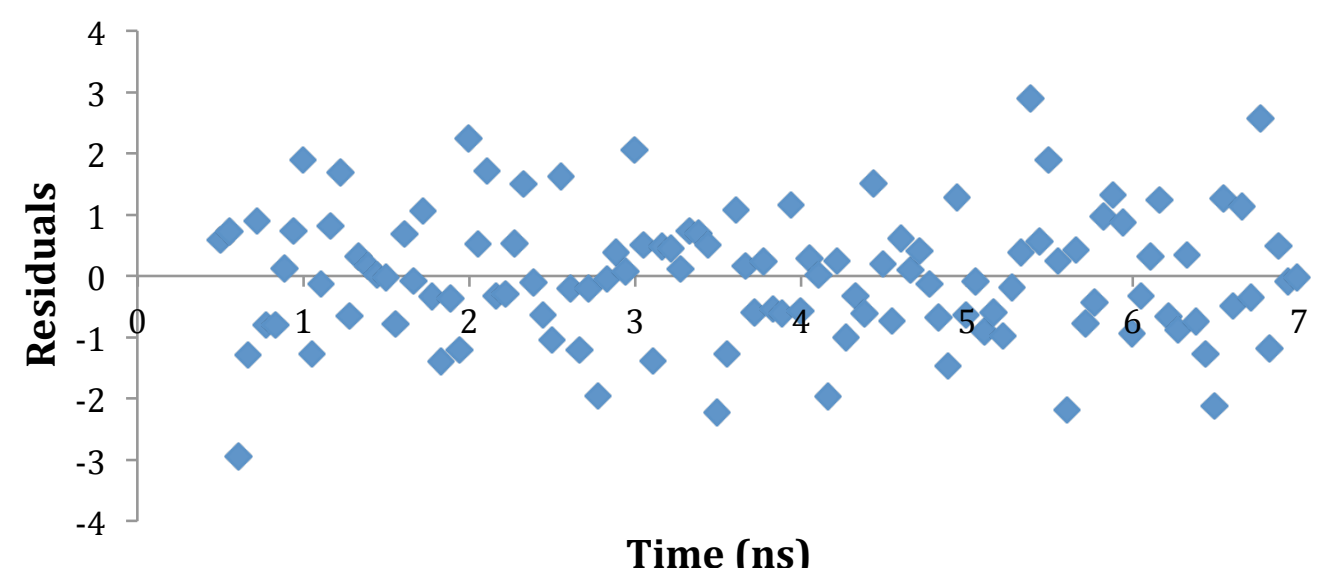

Time (ns)

Figure 40: Residual distribution demonstrates a random spread around zero indicating adequate fitting $\left(\chi^{2}=1.08\right)$. 


\section{RESULTS: DEMONSTRATION OF MULTIFOCAL MULTIPHOTON FLIM}

The multifocal generation and optimization approaches explained in section 10 and 3 , were produced over various stages throughout the development of the system. The initial multifocal generation techniques were developed independently from the SPAD detection as these were generally monitored in the Fourier plane of the SLM using a CMOS camera. The optimization, such as alignment and selective beam scanning was produced with the CMOS-SPAD detection in the descanned detection. However, there were incremental development phases such as the initial setup in the nondescanned detection, which served to calibrate the scan size and provide the first image using the multifocal excitation with the SPAD array architecture via stage-scanning.

Non-descanned detection has various advantages over descanned detection (Section 2). Normally, non-descanned detectors would be placed in the reprojected pupil plane of the objective. Non-descanned detection was performed by re-imaging stationary beamlets whilst operating the system in stage scanning mode. Unfortunately, acquisition speed suffers as a result. As the purpose of parallelising detection is to produce a high speed FLIM system, descanning the emission onto the active areas is preferred. Contrary to an array of PMTs, the SPAD array is a compact detector in which the total 1024 detectors occupy $1.6 \times 1.6 \mathrm{~mm}$. Large numbers of detectors permit a significant increase in the imaging beamlets per TCSPC detector. However the small active area of each SPAD does not permit high-speed acquisition in the direct detection. Previous reports analyse the disadvantages of descanned detection which mainly consist in a degradation of collection efficiency as a function of depth (Diaspro et al., 2006). As the samples imaged are either thin or optically transparent non-descanned detection is not a severe drawback. Increased scattering such as in a tissue sample would result in the focal volume position shift similar to a Tscherning/Shack-Hartmann WFS. Scattering from thick samples would result in misaligned beamlets on the detector, therefore not producing images in the respective misaligned detector segment. 


\subsection{BEAM SCANNED MULTIFOCAL MULTPHOTON FLIM: DESCANNED DETECTION}

In order to fully access high frame rate FLIM, stage scanning is sub-optimal. As was discovered in the early years of confocal microscopy, live cells and tissues do not respond well to oscillatory motion which ultimately would cause potential imaging artefacts. Furthermore, the inertia of any microscope stage is such that resonances are approached rapidly when we consider line pixel and line speeds to achieve scans on the $1 \mathrm{~Hz}$ or less scale. Therefore, the design for the multifocal multiphoton system incorporates beam scanning in the form of two optically relayed galvanometers and the descanned detection of the fluorescence to produce stationary emission beamlets onto the detector array. Figure 36 previously demonstrated the alignment approach for the multifocal generation method of choice (Section 10.6). The weighted GS algorithm (Figure 37) was used throughout this section.

Demonstrated in this section is the optical resolutions (Section 14.2: Figure 41), WGS uniformity improvement applied to a fixed sample (Section 14.4: Figure 43), selective multifocal scanning to generate an optical ROI (Section 14.5: Figure 44) and an evaluation of optical crosstalk (Section 14.5: Figure 45) along with highspeed multifocal FLIM in fixed Convallaria majalis (Section 14.3: Figure 42), live in vitro GFP-CAAX MCF7 cells (Section 14.6: Figure 46) and live in vivo imaging of RhoA biosensor in Zebrafish (Section 14.7: Figure 49). All final images have been corrected for overlapping pixels and scanning artefacts (Section 13: Figure 38) and analysed using a mono-exponential Levenberg-Marquardt fit in TRI2 (Section 13.2: Figure 39). 


\subsection{OPTICAL RESOLUTION}

In order to determine the optical imaging resolution of the system, a $40 \mu \mathrm{m}$ z-stack of $100 \mathrm{~nm}$ fluorescent sub-diffraction limited beads was acquired over 27 minutes (40 s per $\mu \mathrm{m}$, producing lateral and axial FWHM of $0.48 \pm 0.03 \mu \mathrm{m}$ and $1.1 \pm 0.04 \mu \mathrm{m}$ respectively.

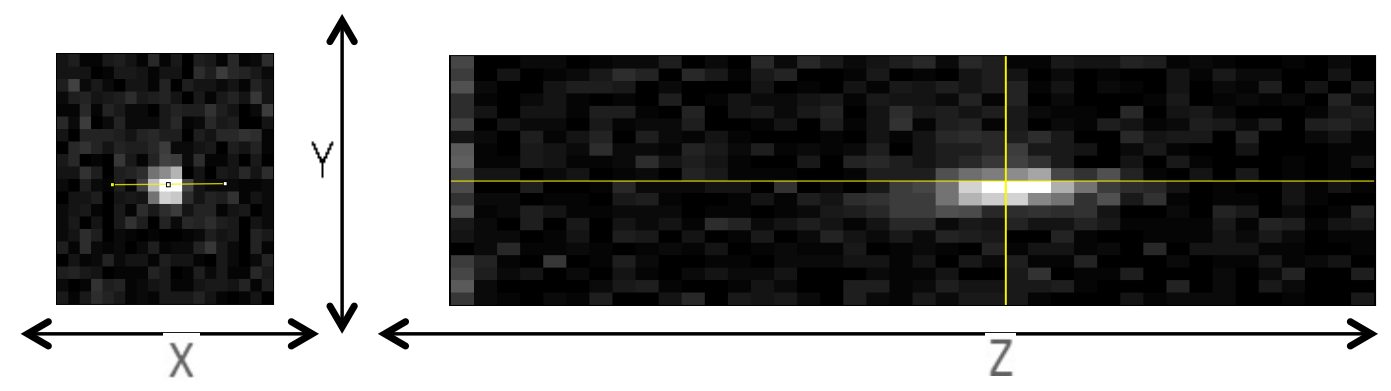

Lateral Profile 100um Bead

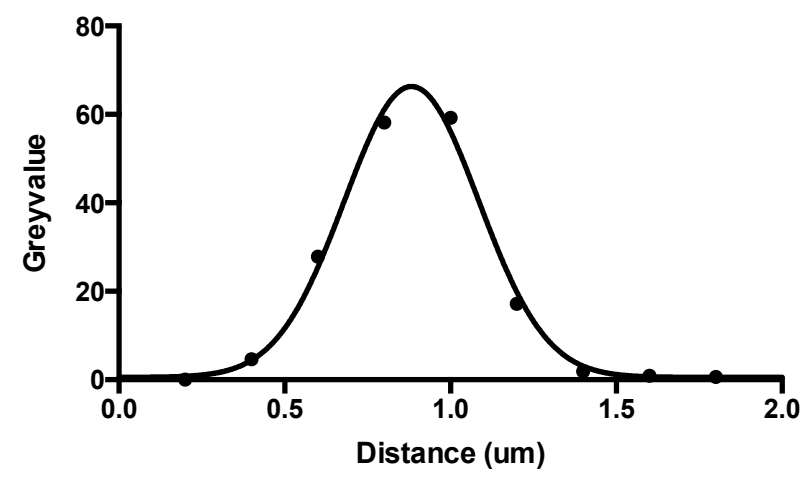

Axial Profile 100um Bead

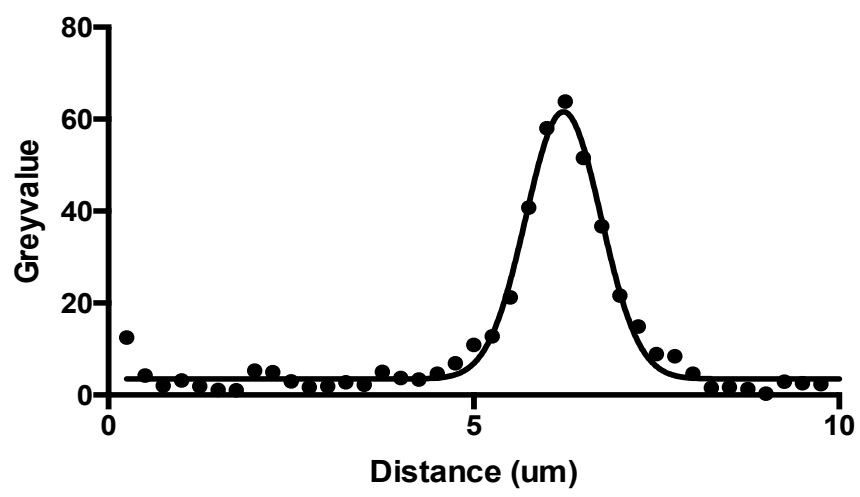

Figure 41: Lateral (XY, Top Left) and axial (YZ, Top Right) acquisitions of a $100 \mathrm{~nm}$ fluorescent bead (contrast was modified to aid visualization). The respective line profile and Gaussian fit (bottom) confirms diffraction-limited performance in the lateral and axial directions. 


\subsection{MULTIFOCAL FLIM USING FIXED SAMPLE}

The fixed Convallaria majalis sample (Leica) (Figure 42) stained with Safranin Red and Fast Green fluorophores imaged with $900 \mathrm{~nm}$ excitation in a square 25 beamlet array was used to generate lifetime contrast (Esposito et al., 2011). The aligned and cropped $10 \mu \mathrm{m}$ stack was acquired at $500 \pm 15 \mathrm{~nm}$ over a total of $200 \mathrm{~s}$ (20s per $\mu \mathrm{m})$. Observed lifetime variations are consistent with standard TCSPC imaging.
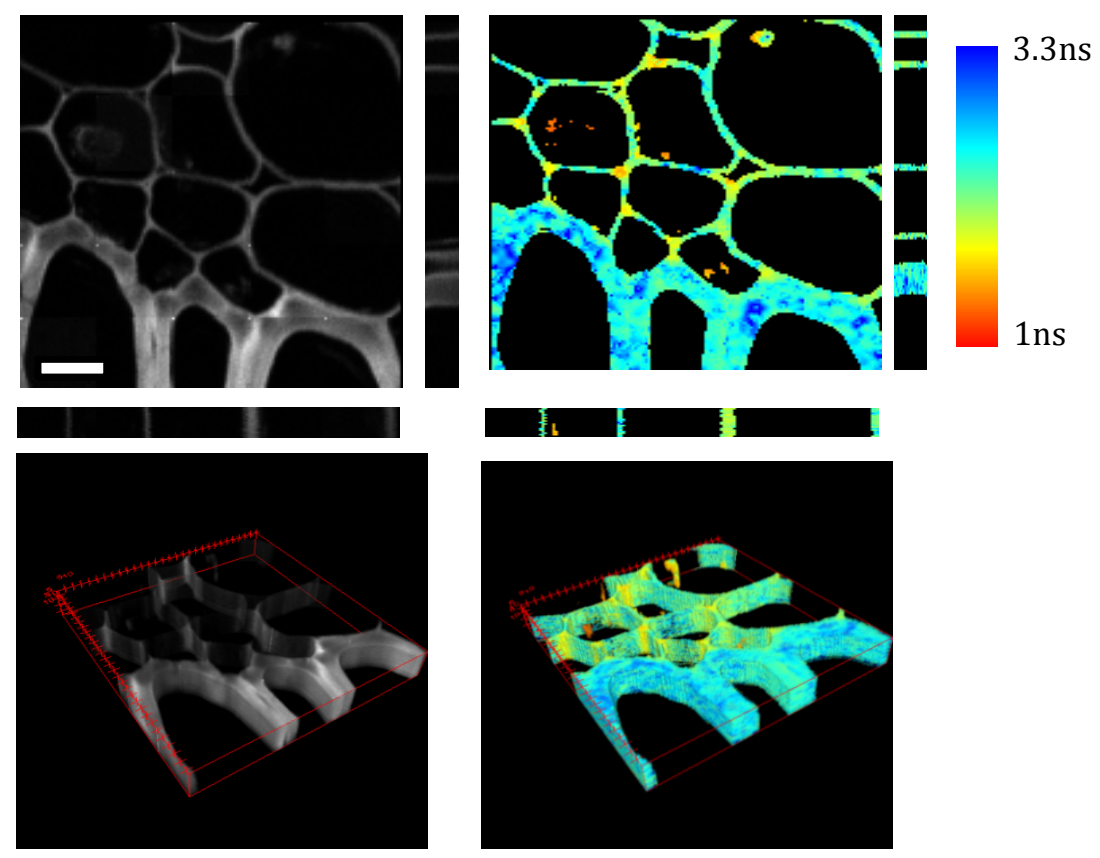

Figure 42: Demonstration of intensity (left) and lifetime contrast (right) using a fixed Convallaria Majalis (Leica) sample. The $10 \mu \mathrm{m}$ z-stack was acquired using 25 beamlets in $200 \mathrm{~s}$. Respective XZ/YZ midway projections at the midway point and $3 \mathrm{D}$ reconstruction shown. Scale bar $10 \mu \mathrm{m}$

\subsection{WEIGHTED GERCHBERG-SAXTON ILLUMINATION}

As previously demonstrated in Figure 28, the GS algorithm produces variable excitation across the multifocal intensity pattern. In order to improve uniformity a weighing coefficient is including in the projected target intensity (Figure 37). The WGS was applied to the illumination pattern demonstrated throughout this chapter. Figure 43 shows the improvement in uniformity achieved in a fixed 
Convallaria majalis sample. The WGS was pre-determined using a green fluorescent block with a $5 \times 5$ illumination pattern (Figure 37).
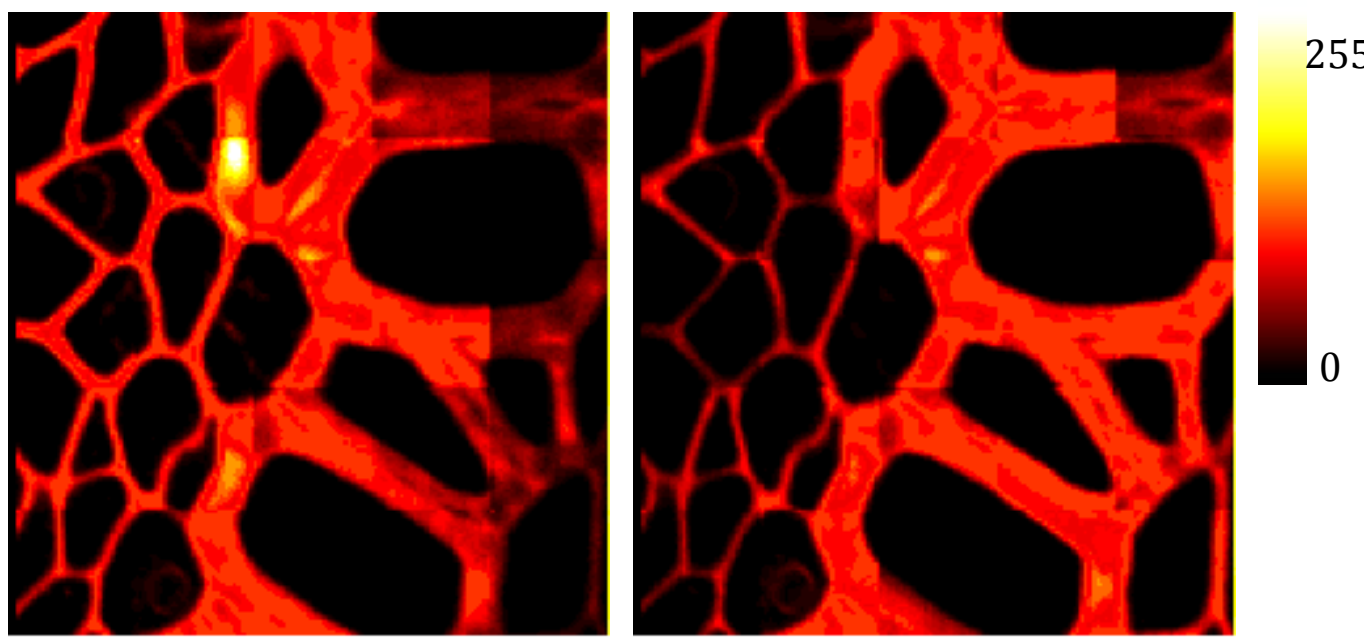

Figure 43: Multifocal (25 beamlets) fluorescence intensity image of Convallaria majalis taken using $800 \mathrm{~nm}$ excitation using the standard GS algorithm (left) and the WGS with a Red Hot LUT (Image J). As expected, the intensity distribution is more uniform in the WGS.

\subsection{SELECTIVE BEAMLET SCANNING}

The alignment procedure (Section 12.2.2) provided great insight regarding the capability, flexibility and dynamic range provided by the holographic beam projection methodology. As a means to further explore the underling prospects, a selective beamlet scanning approach was developed.

Effectively each beamlet scans a square segment of the image plane. As such, 25 beamlets in a $5 \times 5$ configuration scan a region of $62.5 \times 62.5 \mu \mathrm{m}$ or $12.5 \times 12.5 \mu \mathrm{m}$ each, and assuming equal intensity distribution, $\frac{1}{25}$ of the total intensity. However in the case of a region-of-interest of $500 \mu \mathrm{m}^{2}$ (i.e. cell) located in the centre of the image plane (37.5 $\times 37.5 \mu \mathrm{m}$ area), distributing equal amounts of power to nonpertinent beamlets serves solely to misuse approximately $\frac{2}{3}$ of the power for no signal. Therefore to irradiate solely a ROI, the GS algorithm can be recalculated excluding illumination of non-relevant beamlets. This provides the user complete programmability over which segments are imaged, maximizing intensity distribution 
in imaged segments. This ultimately improves acquisition rate due to elevated photon counts; further enhanced due to the quadratic dependency of the twophoton excitation.

Generating selective illumination profiles also serves to evaluate the optical crosstalk of the detectors (Figure 44). Optical crosstalk is generally considered a phenomenon that results in non-intentional illumination of non-pertinent areas/detectors via direct optical paths. However, in SPADs, crosstalk may also arise from one pixel in the array which triggers avalanches in the neighbouring pixels via indirect paths, in a process termed self-triggering (Rech et al., 2008). The avalanche current can be triggered by self-triggering or dark-counting rate can result from reflections within the pixel, thermal tunnelling or trapping processes inside the semiconductor (Zappa et al., 1996).

Under a fully illuminated beamlet array it is impractical to evaluate scattering or reflections as a consequence of back scattering within the SPAD. As such it becomes useful to evaluate the optical crosstalk by illuminating detectors individually or selectively interspersed (Figure 44). Previous reports (Guerrieri et al., 2010; Villa et al., 2012) have demonstrated that crosstalk is not a significant issue for CMOS pixels with similar pitch and SPAD diameter in the orders of a few $\mu \mathrm{m}$. In this configuration, optical effects (ghosting of the pattern, retroreflections etc.) may still indicate crosstalk and must still be considered. Generating a selective pattern and monitoring the detection of the various non-illuminated detectors, with a subtracted background served to quantify the optical crosstalk $(0.2 \pm 0.1 \%)$ (Figure 45). Therefore it possible to assume that if any back reflections or ghost patterns are being generated, these are not detected in the active area of the detectors. As the optical crosstalk is negligible, the detectors act independently without influencing the dark-count rate of the surrounding pixels. 
Applied Pattern
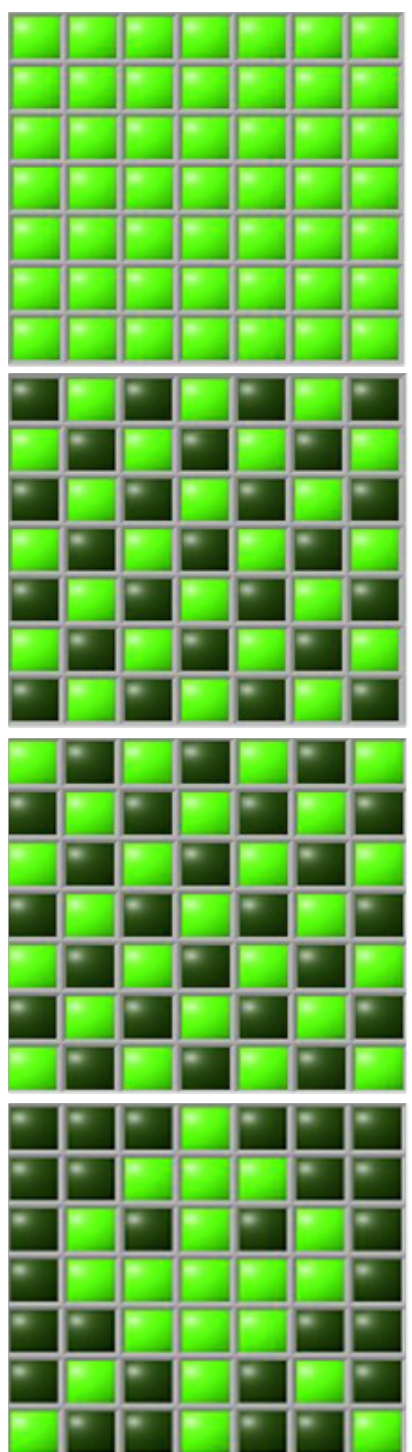

Intensity
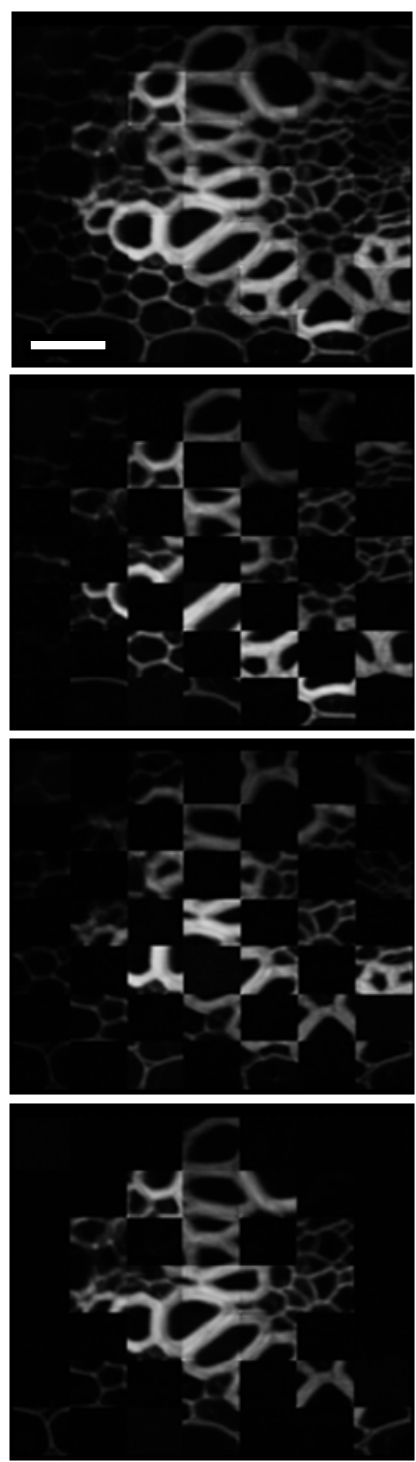

Lifetime

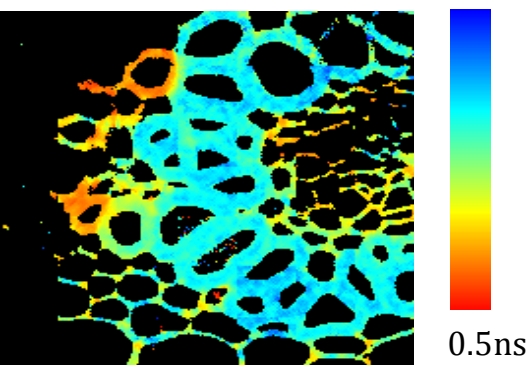

Figure 44: Selective beam scanning employed. The GS algorithm allows for fluorescence to be imaged on a pixel-by-pixel basis and therefore it is possible to quantify the optical crosstalk. The first column shows the selected pattern, the intensity and subsequent lifetime calculation is shown in columns 2, 3 respectively. Scale bar $15 \mu \mathrm{m}$ 

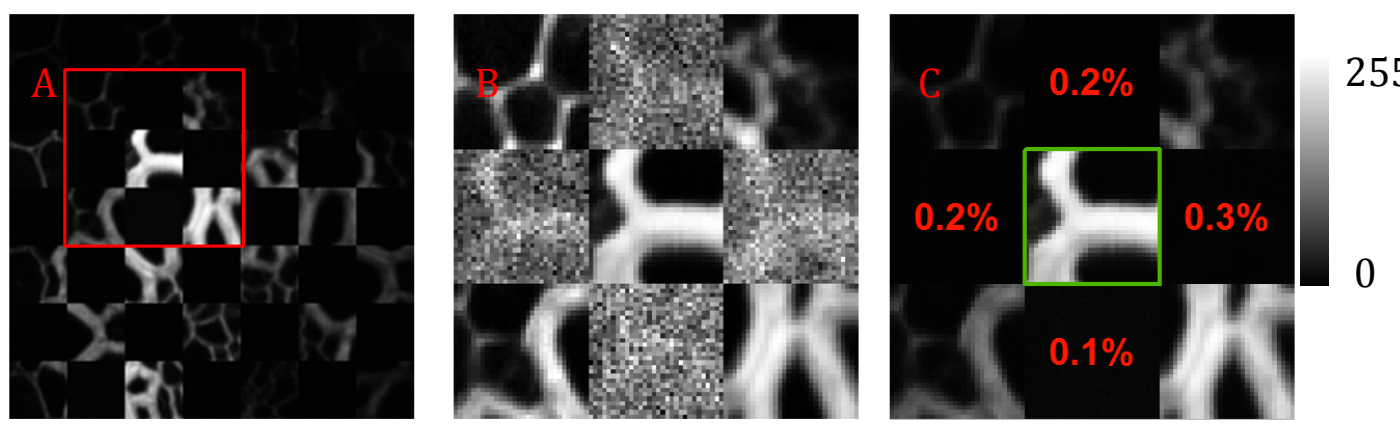

Figure 45: ROI of selective beam scanning $(A)$ with normalized images demonstrate crosstalk (B). Analysis demonstrates a $0.2 \pm 0.1 \%$ optical crosstalk in non-

illuminated detectors (C).

It is possible to test the optical crosstalk using an alternative illumination method, such as the Huygens-Fresnel approach. The outcome however would result in significant misuse of the excitation. Fresnel lens patterns are added to a blaze grating, used to separate the relevant illumination from the remaining orders produced by the SLM diffraction. Therefore creating a selective beam scanning would be a simple task of masking the appropriate segment either on the full hologram or the Fresnel lens pattern for that area. Eliminating the Fresnel lens pattern segments before adding to the blaze could result in deterioration of the SNR due to increased scattering and would provide an unnecessary increase in photobleaching. Masking segments completely would avoid these issues; however either method results in an inefficient management of the excitation intensity. Applying an extended Fresnel pattern to occupy the masked region would improve efficiency, however would generate increased complexity due to the adaptive correction for the elongated projection. Creating an optical ROI (or various ROI within the same image) with the GS algorithm is a matter of altering the desired target intensity by removing non-relevant beamlets, therefore increasing count rate/acquisition speed. This serves as a simple task for GS algorithm in comparison to the Fresnel approach.

\subsection{MULTIFOCAL FLIM IN VITRO}

Cytoplasmic signalling is a fundamental process for cellular functions. CAAX is a protein motif in which $C$ represents the cysteine residue, $A A$ are two aliphatic 
residues and X (Gao et al., 2009). CAAX is involved in a series of cellular signalling processes and regulatory events (Hancock et al., 1990). Primarily found in cytoplasmic surface, CAAX plays a role in proliferation, differentiation, nuclear stability, embryogenesis, spermatogenesis and apoptosis (Boucrot and Kirchhausen, 2008; Choy et al., 1999; Gao et al., 2009). FRET-FLIM has been shown upon stimulation from epidermal growth factor (Rocks et al., 2005; Van Rheenen et al., 2007). A $5 \times 5$ beamlet array was used to acquire a $5 \mu \mathrm{m}$ z-stack of live MCF7 breast cancer cells transfected with EGFP-CAAX (Figure 46) imaged though a glass-bottom well plate. The average lifetime measured was $2.4 \pm 0.11 \mathrm{~ns}$ acquired for $20 \mathrm{~s}$ per $\mu \mathrm{m}$ image slice. Cell membrane can clearly be observed in Figure 46 in addition to EGFP overexpression in the nucleus.

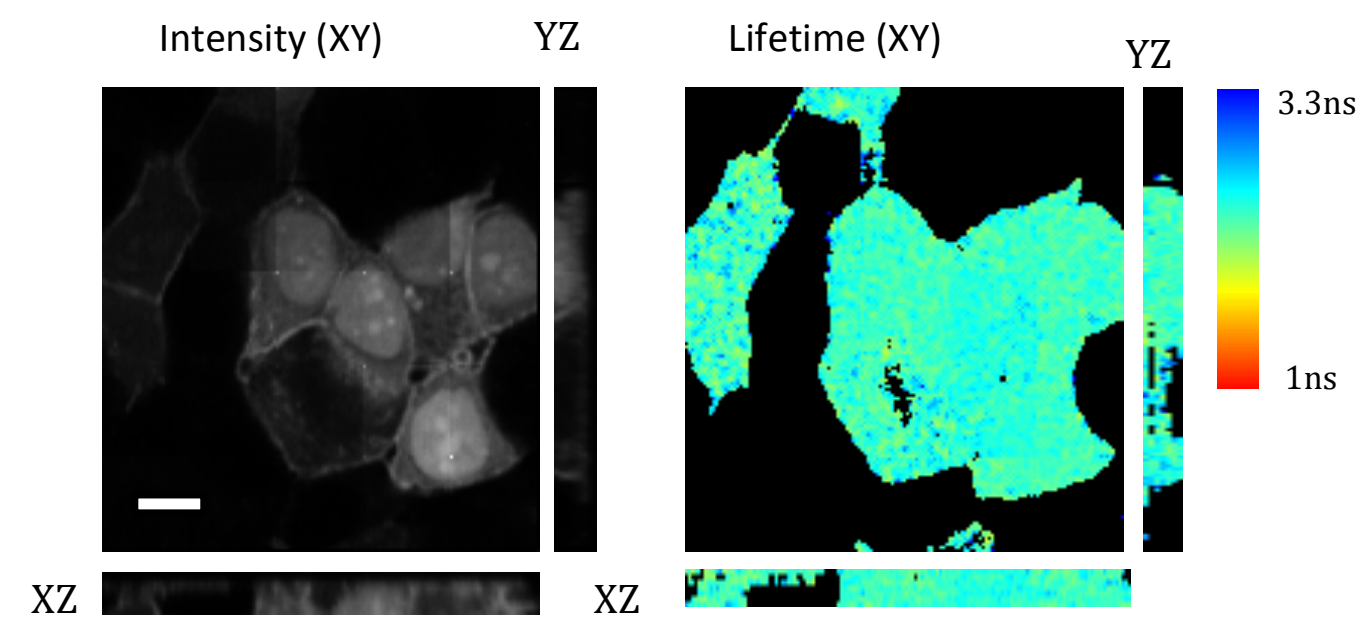

Figure 46: Intensity (left) and lifetime (right) of MCF7 cells transfected with GFPCaaX $5 \mu \mathrm{m}$ z-stack acquired using 25 beamlets exhibiting the respective XZ/YZ axial projections at the midway point and 3D reconstruction. 3D images of EGFP-CAAX cells acquired using 25 beamlets with $900 \mathrm{~nm}$ excitation demonstrate the expression of EGFP throughout the cell. Scale bar $10 \mu \mathrm{m}$. 
Average Fluoresecence Lifetime distribution in EGFP-

CaaX cell

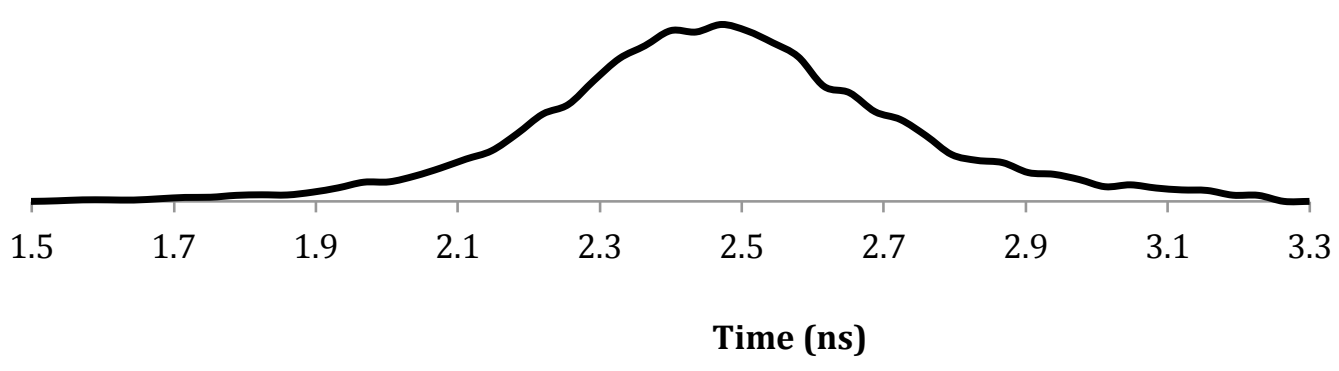

Figure 47: Fluorescence lifetime distribution of the MCF7 EGFP-CAAX cell. As the cell contains only GFP, the average lifetime value of $2.4 \pm 0.11 \mathrm{~ns}$ is consistent with previously measured datasets.

MCF7 cells expressing FRET standards comprising of EGFP and mRFP1 (Matthews et al., 2012) separated with various length amino acid linker sequences provided by Dr. James Moneypenny (Prof. Ng Lab) were acquired for 10 seconds (Figure 48). FRET efficiencies calculated in agreement with Eq. 10 (Chapter 1, Section 6) increase with shorter linker sizes. Comparison with single beam TCSPC (Ameer-Beg et al., 2002) is shown in Table 5 (Poland et al, 2014 Submitted). 

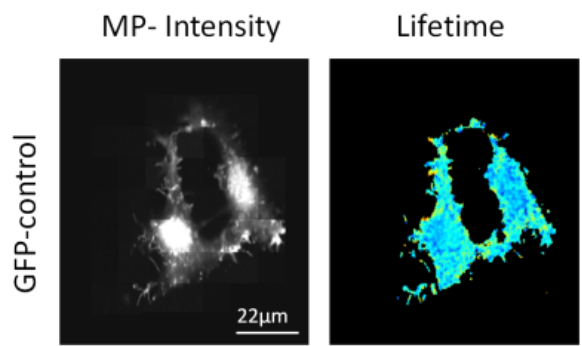

Composite
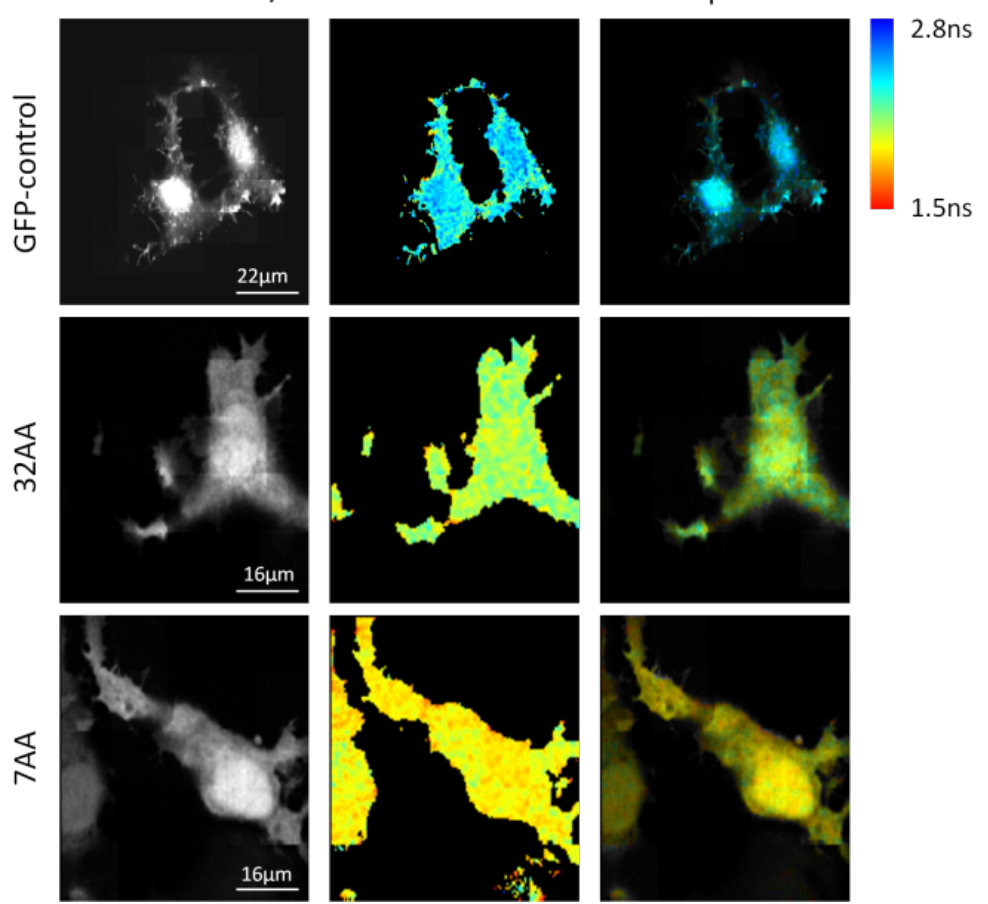

Figure 48: Intensity, lifetime and composite images of MCF7 cells expressing EGFP connected to mRFP using amino acid chains of various sizes compared with MCF7 cells expressing EGFP alone.

Table 5: FRET efficiencies registered for various amino acid chain lengths

\begin{tabular}{|l|c|c|c|c|}
\hline Transfection & GFP_CaaX & GFP_32aa_RFP & GFP_19aa_RFP & GFP_7aa_RFP \\
\hline $\begin{array}{l}\text { FRET } \\
\text { efficiency } \\
\text { (Single beam } \\
\text { TCSPC) }\end{array}$ & 0 & $0.163 \pm 0.003$ & $0.199 \pm 0.001$ & $0.244 \pm 0.003$ \\
\hline $\begin{array}{l}\text { FRET } \\
\text { efficiency } \\
\text { MM-FLIM }\end{array}$ & 0 & $0.154 \pm 0.002$ & $0.184 \pm 0.002$ & $0.224 \pm 0.005$ \\
\end{tabular}




\subsection{HIGH SPEED IN VIVO FLIM}

Ultimately being able to visualize, monitor and quantify interactions in vivo serves to more accurately verify biological hypotheses. Imaging protein-protein interaction or protein-folding activity in Zebrafish using FRET-FLIM allows the determination of the activation state of key biochemical pathways under physiological conditions (Ashworth and Brennan, 2005; Kardash et al., 2011; Kumar et al., 2011; McGinty et al., 2011). Over the last few decades Zebrafish have been used regularly to study cancer models, as these can spontaneously develop a large variety of tumours (Matthews, 2009). In terms of optical imaging Zebrafish are particularly pertinent as these are transparent embryos and have ex-utero development of the embryo (Appendix B) (Kardash et al., 2011). Developed and supplied by Dr Claire Wells group the Zebrafish, incubated at $28^{\circ} \mathrm{C}, 200 \mu \mathrm{M}$ Phenylthiocarbamide was added daily to the embryos after the first day of fertilization. On the second day post fertilisation (dps) embryos were anaesthetised in $0.5 \mathrm{mM}$ tricaine and A-375MM cells expressing a modified RhoA biosensor containg a mTFP1 donor and circularly permuted YFP acceptor was injected into the pericardial cavity (Fritz et al., 2013). After 1 hour at $28^{\circ} \mathrm{C}$ post-injection the embryos were incubated at $34^{\circ} \mathrm{C}$. On the $5^{\text {th }}$ day these were embedded in $1.5 \%$ low melting point agarose (containing $0.5 \mathrm{mM}$ tricaine) in order to reduce motility and submerged in $25 \mathrm{mM}$ tricaine and imaged immediately. The 3D intensity and lifetime Zebrafish data shown in Figure 49 is the result of 40 acquisitions over a $40 \mu \mathrm{m}$ axial stack acquired using 5 x 5 beamlet excitation. Optical sections demonstrated are obtained every at 1 frame/10s produce a total of 40 images acquired within $400 \mathrm{~s}$ with final dimensions of $62.5 \times$ $62.5 \times 40 \mu \mathrm{m}$ (Figure 49), comparable to the acquisition of a single image for typical TCSPC imaging using a single beam scanning approach. A Levenberg-Marquardt mono-exponential fit performed in TRI2 (Section 13) was applied to the data (image-by-image basis) producing the fluorescence lifetime histogram across the acquisition (Figure 50) displaying a FRET efficiency distribution compared to an mTFP1 control (Figure 51). 

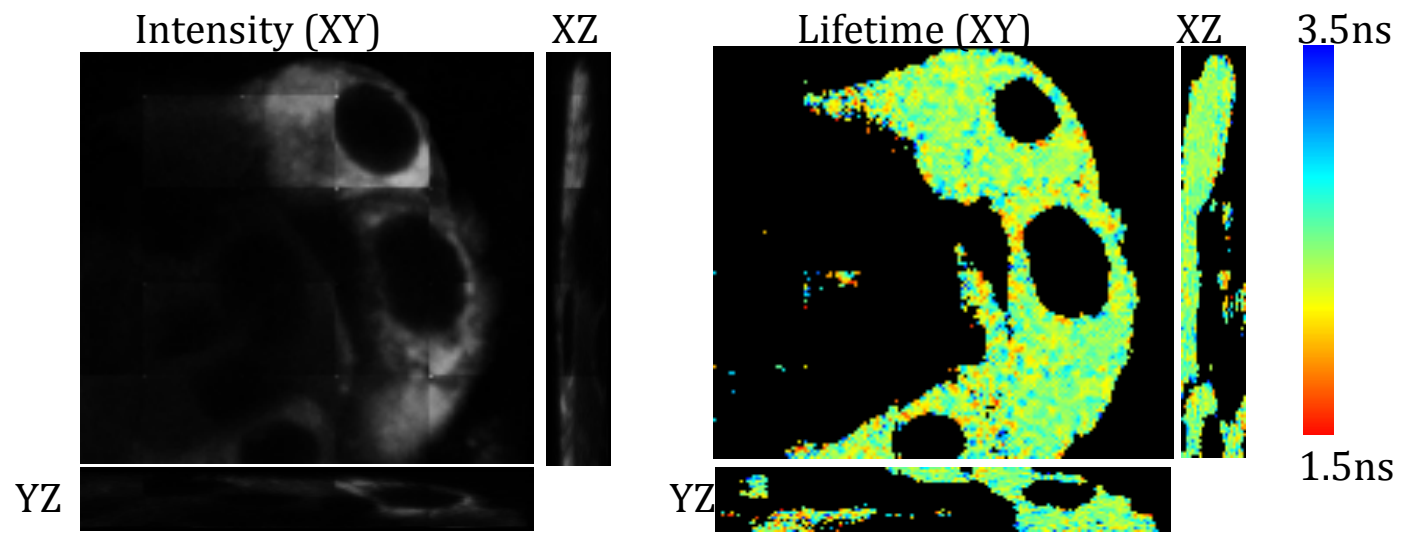

3D Intensity
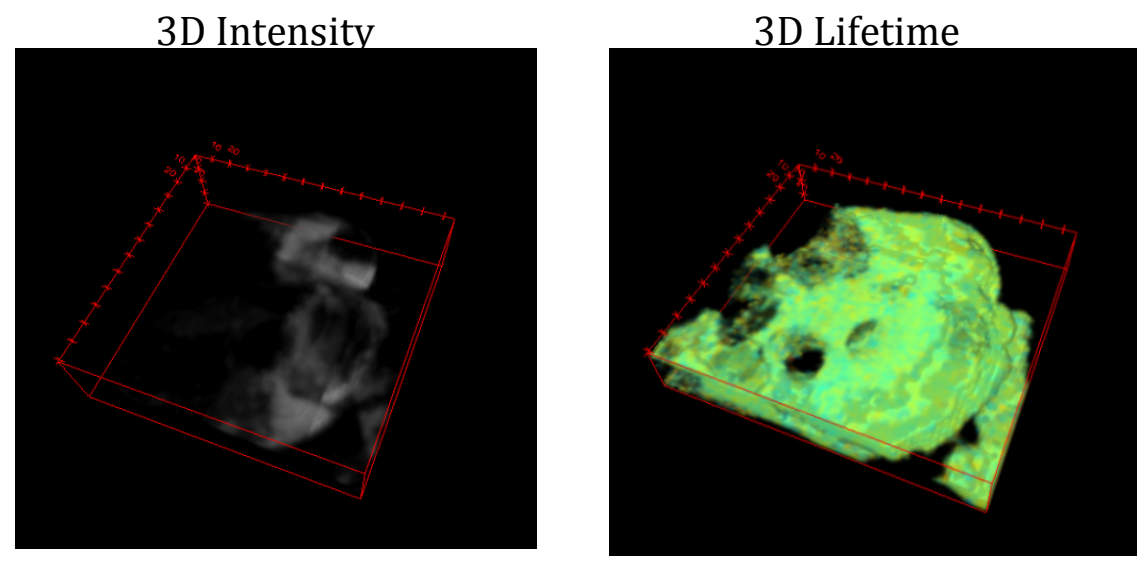

Figure 49: High-speed in-vivo TCSPC MM-FLIM. Intensity (left) and fluorescent lifetime (right) of a 4 day Zebrafish embryo with A-375MM cells expressing a modified Rho-A biosensor injected into the pericardial cavity. The z-stack was produced using 25 beamlets with $900 \mathrm{~nm}$ excitation wavelength at a rate of $10 \mathrm{~s}$ per acquisition. The $62.5 \times 62.5 \times 40 \mu \mathrm{m}$ z-stack exhibits the respective $\mathrm{XZ} / \mathrm{YZ}$ axial projections at the midway point and 3D perspective data. 
Fluorescence Lifetime Histogram (ns)

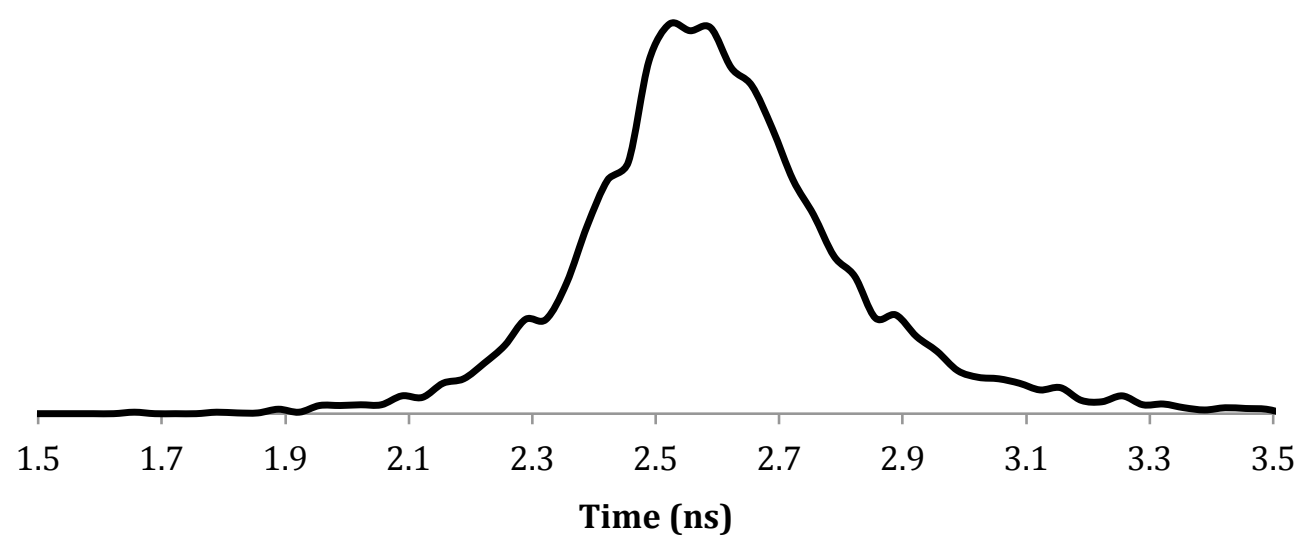

Figure 50: Fluorescence lifetime histogram of the Zebrafish optical stack obtained using a Levenberg-Marquardt mono-exponential fitting.

FRET Efficiency of Zebrafish Stack

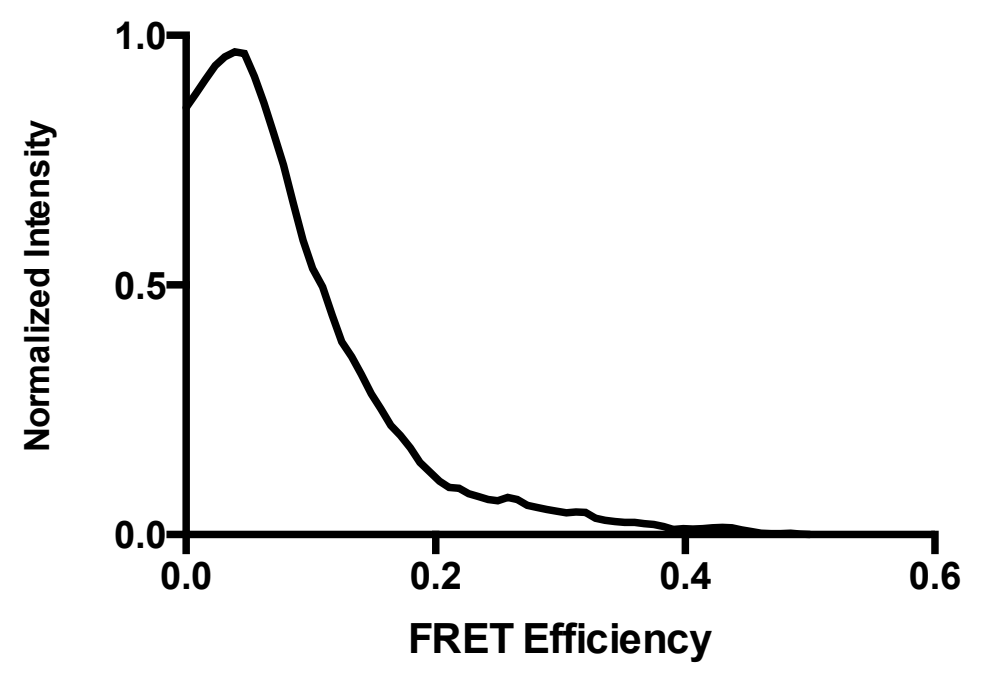

Figure 51: FRET efficiency of the Zebrafish stack. 


\section{DISCUSSION}

The purpose of this project was to produce a highly parallel, fully addressable timeresolved multifocal multiphoton microscope capable of producing fluorescence lifetime images to visualize spatio-temporal interactions of proteins at increased speed. Current multifocal techniques incorporating FRET-FLIM such as the TriM Scope and the spinning disk are either limited by the quantity of beamlets produced (Kumar et al., 2007a), the time resolution provided by detection electronics (Grant et al., 2007; Van Munster et al., 2007) or the amount of detectors (Kim et al., 2007). Section 14 demonstrates the capability of the system to produce rapid acquisitions using TCSPC-FLIM. The scan period of the MM-FLIM system was 10-20s to ensure sufficient counts per pixel for an adequate fit. Producing high-speed FLIM in a 3D structure was demonstrated using Convallaria majalis, MCF7 breast cancer cells transfected with EGFP-CAAX and in vivo Zebrafish pericardial cavity injected with a Rho-A biosensor. The $62.5 \times 62.5 \times 40 \mu \mathrm{m}$ z-stack of the biosensor injected into a Zebrafish's heart cavity was acquired over 400s, comparable to the acquisition of a single image for typical TCSPC practiced. Typical single beam acquisition is collected over a period of 3-5 minutes using a standard beam scanning FLIM with (for example) B\&H SPC830 electronics. Conformational changes or faster alterations of the sample would have been lost within the acquisition period. 3D acquisition of FRET-FLIM visualizations of cells permits high spatio-temporal resolution of a large variety of structures such as tumours, which can be developed in vivo (Appendix B for MIFT-A clustering).

The work presented has been performed in the context of TPM. High-aperture beamlets reduce higher order photo-damage effects through parallelization, while maintaining the many advantages of single-beam TPM, such as superior live-cell compatibility, reduced photobleaching of non-target planes and optical sectioning. The small focal volume also aided in the alignment of the emission with the detection areas. In order to ensure sufficient photon density in each beamlet focus without overheating the LCOS-SLM display $\left(P_{\max } \approx 1 \mathrm{Wcm} \mathrm{cm}^{-2}\right)$ the maximum amount of beamlets was limited to 49 in a $7 \times 7$ configuration $(P \approx 6 \mathrm{~mW}$ per beamlet $)$. The SLM introduces the WGS algorithm's hologram in 
the optical system producing a multifocal array in the Fourier plane of the SLM, which is relayed onto the sample. The flexibility of a holographic method was shown to be important for alignment, selective beamlet illumination, an optical ROI, evaluate detector crosstalk and to reduce heterogeneity of the emission detected via a weighting function. 


\section{FUTURE OF THE PROJECT}

Potential developments and applications including high-content screening, multiwavelength detection, anisotropy, super-resolution (STED), flow cytometry, FCS and single-molecule imaging FRET. The signal may be spectrally split via a dichroic reimaged onto a second detector generating multi-wavelength detection or could be split using a prism and projected onto different detectors within the same SPAD array. The excitation may be changed for a visible laser to reduce costs, in which the confocality can be produced via the small detection area. There are additional issues such as out-of-focus excitation and correct alignment to the focal volume however a confocal excitation source has the potential to produce more beamlets as there is a significantly less thermal constraint on the SLM. This also permits an improvement on current FCS/single-molecule FRET techniques currently practiced (Michalet et al., 2013).

A diffractive optical element (DOE) may replace the SLM. A DOE serves as a thin microstructure pattern to alter the phase of light propagated through it. Properly designed it can manipulate the light to almost any desired intensity profile. Generally fabricated using photolithography (Rogers et al., 1997), the wavefront phase pattern, located in the hologram plane would correspond to the SLM's calculated phase projection. A drawback remains: lack of flexibility. An option to overcome this may include a series of DOEs which can be produced with different patterns corresponding to different Fourier transforms (i.e. checkerboard pattern, $3 \times 3 / 5 \times 5 / 7 \times 7$ beamlets) which could interchange at the user's discretion.

Improvement of the PSF and excitation efficiency in scattering tissue may be performed by adaptive optics. Taking the example of the in vivo sample in which the heart cavity has a dense fluorescently labelled section, a rapid beamlet position optimization procedure may be performed (within reasonable limits) and the difference in displacement between the original positions provided by a fluorescent block and the newly aligned fluorescence can be translated into slopes capable of producing a Zernike pattern for a given sample. 
Enhancement in photon detection efficiency of the SPADs and higher fill factor may also provide a larger scope, while facilitating development. Updating transfer from USB2 to USB3 will provide higher readout rates, expected to be $320 \mathrm{Mbps}$. 


\section{CHAPTER 3- FLIM WITH ADAPTIVE OPTICS}

\section{OVERVIEW}

Adaptive optical correction has been extensively employed to aid visualization of biological samples at increased depth. Distortions to a wavefront from the ideal plane or spherical shape are the principal causes for image contrast and resolution degradation (Booth, 2007). A phase delay can be applied to the excitation illumination to create a corrected wavefront countering the distortions introduced by the instrument and the specimen (Babcock, 1953; Pepper, 1986; Tyson, 2010). In terms of FLIM, the SNR degradation as a function of depth due to the morphology and high diversity of cellular and in vivo samples leads to increased errors in the fit parameters and consequent broadening of the derived lifetime histogram (Figure 4).

A series of approaches have been developed to determine the aberration of the combined system and sample aberrations (Booth et al., 2002; Booth et al., 1998a; Girkin et al., 2009; Marsh et al., 2003; Neil et al., 2000a; Neil et al., 2000b; Schwertner et al., 2004; Wright et al., 2005). Established techniques such as wavefront sensing based on the TWS or SHWFS using a membrane deformable mirror have been used in ophthalmology (Porter et al., 2006), microscopy (Booth et al., 2002; Neil et al., 2000b) and astronomy (Roddier, 1999; Tyson, 2010); direct sensing such as Hill-Climbing, RS (Wright et al., 2005) using a DMM or pupil segmentation (Ji et al., 2010) using a SLM have been developed for microscopy. Representation of the wavefront using Zernike polynomials under a modal reconstruction reduces acute frequency variations but is decomposable in terms of simpler and comparable functions, which are expressed via their coefficients. Discrete representation provides high frequencies due to the more independent nature, but does not permit interpretation in optical terms (Dai, 2008). 
Regardless of the method, the objective is to improve image SNR, contrast and resolution degraded via the system and sample's phase variations. The overall aberration is the sum of the specimen and system aberration components. System aberrations are caused by optical components (for example lenses and an SLM). Particularly, SLM often suffer from poor surface quality, introducing aberrations which degrades a system's capability of performing high-resolution measurements i.e. STED (Gould et al., 2012; Gould et al., 2013) or structured illumination (Débarre et al., 2008). System distortions are static and are assumed to be constant for a given optical configuration. Specimen aberrations are caused by the inhomogeneity in the refractive index. Accurate calculation and projection of a wavefront capable of counteracting phase variations eliminates or reduces aberrations in the objective front focal plane.

The work presented in this chapter consists of a pupil-segmentation based adaptive optical correction multiphoton microscope applied to FLIM. Improvement of SNR increases the accuracy of FLIM in specimens that highly distort the excitation wavefront. Section 1 describes the pupil-segmentation method to obtain the phase corrective wavefront. Section 2 discusses the experimental procedure and optical system used throughout this chapter including the optical design rationale. Section 3 concentrates on the experimental results obtained with the system. System and sample imaging improvement performance permits increased SNR and resolution demonstrated in second-harmonic generation in live zebrafish and oncological histological sections with FLIM, followed by a discussion in section 4 . 


\subsection{PUPIL-SEGMENTATION APPROACH TO ADAPTIVE OPTICS}

The method adopted to counteract the phase variations induced by refractive index mismatches was a direct sensing approach based on pupil-segmentation ( $\mathrm{Ji}$ et al., 2010; Ji et al., 2012; Milkie et al., 2011; Wang and Ji, 2012). The pupil-segmentation approach regionally illuminates the imaging objective's pupil sequentially. The analysis of aberrated rays sequentially provides insight to the various distortions sensed in each section (Figure 52, Figure 53). Refractive index variations generate a position change in the image plane for an illuminated segment of the pupil with respect to the fully illuminated pupil (Figure 52). The concept is relatively straightforward: if the number of sub-apertures generated at the entrance pupil of the imaging objective is sufficiently large, a diffraction-limited focus is achieved at increased depths. In order to generate a tiled pupil segment, a blazed grating function is selectively projected onto a SLM's micro-display. The area of the blazed function is directly correlated to the segmented area of the pupil. Therefore the displacement of a particular image obtained is a consequence of uncorrected local aberrations. Once the displacements for all sub-regions are calculated, a wavefront reconstruction fitted to Zernike polynomials is performed. The corrective wavefront is a summation of the Zernike polynomial coefficients. This approach can be considered a hybrid between a wavefront sensor approach and direct sensing. It does not contain a wavefront sensor that sections the beam; however it directly segments and probes an area analogous to a wavefront sensing lenslet probing zone. As the segmented blaze hologram does not result in beamlets, this imaging method has the benefit of not suffering from crosstalk/crossover. Previous reports using pupil-segmentation have shown diffraction limited imaging at a depth of $450 \mu \mathrm{m}$ in fixed mouse cortical slices (Ji et al., 2012). 


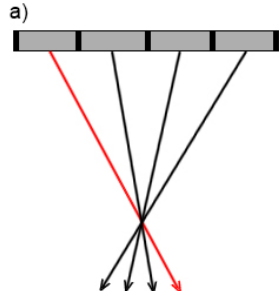

c)

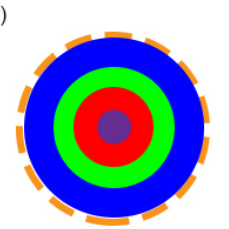

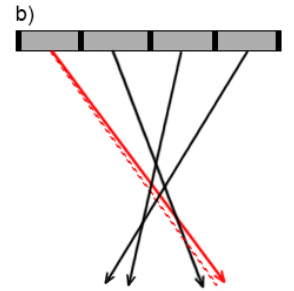

d)

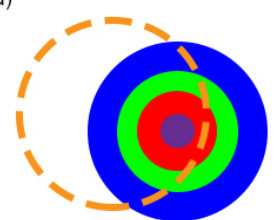

Figure 52: Schematic of illumination procedure using the pupil-segmentation algorithm. The ideal focus translates ( $a$ and $c$ ) to all rays intersecting at the same focal spot. Segmentation of the pupil permits analysis of aberrated rays; these translate to a shift of the image plane ( $b$ and $d)$.

The experimental procedure for the simplest case, such as a bead immersed in a uniform media, starts with the acquisition of a first image with the blaze grating occupying the entire hologram region. The centre-of-mass of the bead under noncorrected conditions can be calculated from the acquired image. The following steps consist of imaging the bead repeatedly while illuminating through individual segmented tiles filling the pupil. As the pupil is sectioned, the centre-of-mass of the acquired bead images shift. The value for each pupil tile is subtracted from the bead's initial position to obtain the slope displacement necessary for the zonal reconstruction (Figure 53). Applying the corrective wavefront to the blaze grating, rays originating from the entire pupil intersect at the same focal spot. 


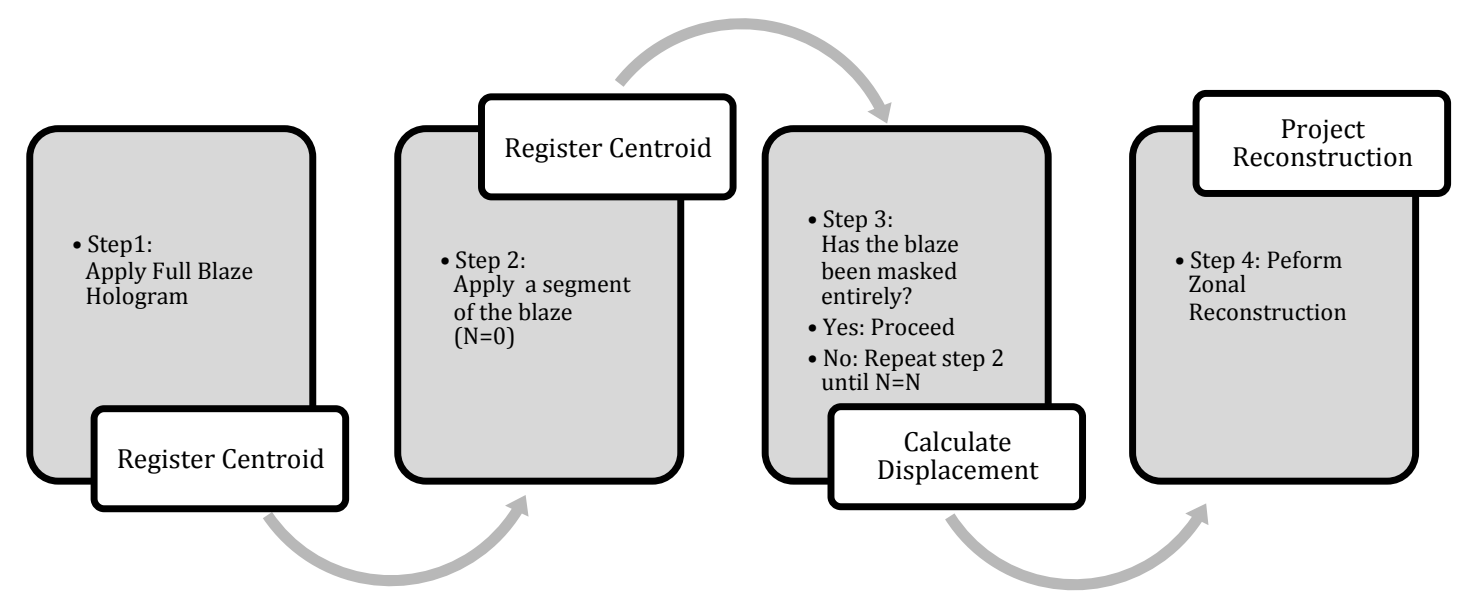

Figure 53: Flow Chart for Pupil-Segmentation based on Zonal Reconstruction, in which $\mathrm{N}$ equals the number of masked regions

In contrast to a DMM in which all actuators have interdependency, an SLM can be used to produce pupil segmentation owing to each pixel being independent of the others. The projection of individual segments of the blaze function onto the SLM's microdisplay generates completely independent illuminating regions. By masking the blaze hologram exposing only a section to the illumination, the non-modulated illumination is not projected into the $1^{\text {st }}$ diffractive order. The area of the segmented blazed function is directly proportional to the segmented area of the pupil, resulting in a decrease of the effective NA of the objective. A significant increase in excitation power is needed to compensate for the decrease in effective NA of the objective. Illuminating only a section of the back aperture of the imaging objective results in a severely elongated beamlet focus, generating ghost images contributions from fluorescent structures beyond the region of the excitation. In three-dimensional labelled structures, accurate centre-of-mass calculation has been shown to be very challenging (Ji et al., 2010; Milkie et al., 2011). Analogous to the area of lenslet in the TWS/SHWS, a larger pupil segment provides a more accurate centre-of-mass calculation. Smaller pupil segments, however, provide a more accurate wavefront reconstruction. To reduce this limitation, a method which relies on stepping the segment sequentially - creating overlapping regions in the reconstruction is performed (Espinosa et al., 2010; Ji et al., 2010; Milkie et al., 2011) (Section 18.3). Overlapping regions provide intermediate equally contributing slope 
calculations in the zonal reconstruction. Fitted using a Levenberg-Marquardt routine, Zernike polynomials with the respective coefficients calculated are applied to the SLM's initial blaze grating function, producing the final corrective wavefront.

In section 19.1 mathematical modelling of Zernike polynomials and subsequent fitting provides a basis of comparison, via the root-mean-squared deviation, between reconstructed and produced wavefronts using a segmentation method. Reconstruction is performed using a Zonal reconstruction and subsequently fitted to Zernike polynomials using a Levenberg-Marquardt fit. A theoretical approach has been undertaken, in addition to a farfield reconstruction algorithm, to simulate the effects of the segmentation procedure on aberrations in the Fourier plane.

In order to demonstrate wavefront correction in our custom built TPM-FLIM system, a Zernike polynomial approach using an overlapping stepped pupilsegmentation, is applied to correct for static system and variable sample aberrations. The optical imaging system with aberration correction is demonstrated by the application of Zernike characterization of an oncological tissue slice with FLIM in addition to Zebrafish using second harmonic (Section 20). These sampleinduced distortions are determined in conjunction with already calculated system aberrations. Zernike coefficients of the static system aberrations were determined using fixed fluorescent beads, under minimum aberration conditions (Section 19.2). 


\section{EXPERIMENTAL PROCEDURE}

In this section we describe the optical system developed for multiphoton fluorescence lifetime imaging with adaptive optics control by pupil segmentation. In The optical design is described in Section 2.1, Section 2.2 describes the electronic control, Section 18.2 the SLM calibration and Section 18.3 describes the experimental procedure for acquiring aberration data.

\subsection{OPTICAL DESIGN}

The optical design (Figure 54) incorporates a few common features to the MegaFLI system, such as the galvanometer scanning and the SLM located in the hologram plane. A Holoeye Pluto NIR2 SLM is used to project segmented portions of the blaze function required to selectively illuminate the back aperture of the objective (Nikon x25 1.1 NA) and add the respective AO correction. Laser light from a Ti: Sapphire laser system [Spectra-Physics MaiTai] is expanded to overfill the SLM. The appropriate phase pattern is calculated and projected onto the device's microdisplay. A blaze phase grating is used to efficiently project the pattern into the first order diffraction pattern. A Fourier-plane aperture filters out the remaining orders consequence of imperfections in the SLM's phase response. The pattern is sequentially relayed through a set of $x$ and $y$ galvanometer scanners onto the back aperture of the objective within a Nikon Ti-E microscope body. Sample fluorescence generated is then collected and re-directed via a dichroic mirror [Semrock]. The fluorescence is spectrally split into the red and green components which are imaged onto separate B\&H hybrid PMT detectors (HPD) (Becker et al., 2011).

The optical train initiates with a $\times 2$ expansion via the $L_{1} / L_{2}(50$ and $100 \mathrm{~mm})$ (Figure 54). A second telescope $L_{3} / L_{4}(50$ and $300 \mathrm{~mm}$ ) illuminates the SLM with a $12 \mathrm{~mm}$ collimated beam at an $18.5^{\circ}$ angle such that the blaze function occupying an area of $8 \times 8 \mathrm{~mm}$ on the SLM produces a perpendicular first diffractive order. Located at a $400 \mathrm{~mm}$ distance from $L_{5}\left(f\left(L_{5}\right)=400 \mathrm{~mm}\right)$, is reduced by a factor 
of 4 due to $L_{6}(f=100 \mathrm{~mm})$, producing a lateral length of $2 \mathrm{~mm}$. A further $\times 2.5$ magnification is produced ( $L_{7} / L_{8}, 200 \mathrm{~mm}$ and $500 \mathrm{~mm}$ ), part of the initial setup built intended for a standard TPM. The setup contains a pair of mirrors ( $F M_{1} /$ $F M_{2}$ ) mounted on flip mirror mounts [FM90/M Thorlabs], which serve to bypass the SLM, creating a standard TPM.

Lenses $L_{9 / 10}\left(f\left(L_{9 / 10}\right)=50 \mathrm{~mm}\right)$ located between the $x$ and $y$ galvanometer scanner mirrors generating a $4 f$ relay. The scan lens $L_{11}\left(f\left(L_{11}\right)=75 \mathrm{~mm}\right)$ provides an additional $\times 2.7$ magnification of the excitation with the tube lens $L_{12}$ $\left(f\left(L_{12}\right)=200 \mathrm{~mm}\right)$, located within the Nikon Ti-E microscope body. A square with $13.3 \mathrm{~mm}$ length is projected on the $16 \mathrm{~mm}$ diameter pupil therefore underfilling the objective; consequently reducing the effective NA to 0.9. The fluorescence generated is separated from the IR/NIR excitation via a dichroic filter [Semrock] and collimated using $L_{13}\left(f\left(L_{13}\right)=75 \mathrm{~mm}\right)$; the second dichroic [Semrock] spectrally splits the fluorescence onto a pair of Hybrid PMTs (B\&H).

Hybrid photodetectors are comprised of a photocathode mounted on top a vacuum tube followed by an APD operated in its linear gain regimen (Suyama et al., 1997). The photocathode has a high acceleration voltage $(8 \mathrm{kV})$ to inject photoelectrons into a silicon avalanche diode. The APD operates by generating a larger number of electron-hole pairs in the silicon as previously described (Chapter 2, Section 9). This configuration has an improved counting efficiency over a conventional PMTs (Fukasawa et al., 2008). As significant gain is obtained in a single step, these devices produce single-photon pulses with a narrow amplitude distribution and reduced afterpulsing. The sensitive area of a Hybrid PMT (8mm diameter) is sufficiently large to be used in the non-descanned detection (Michalet et al., 2008). 


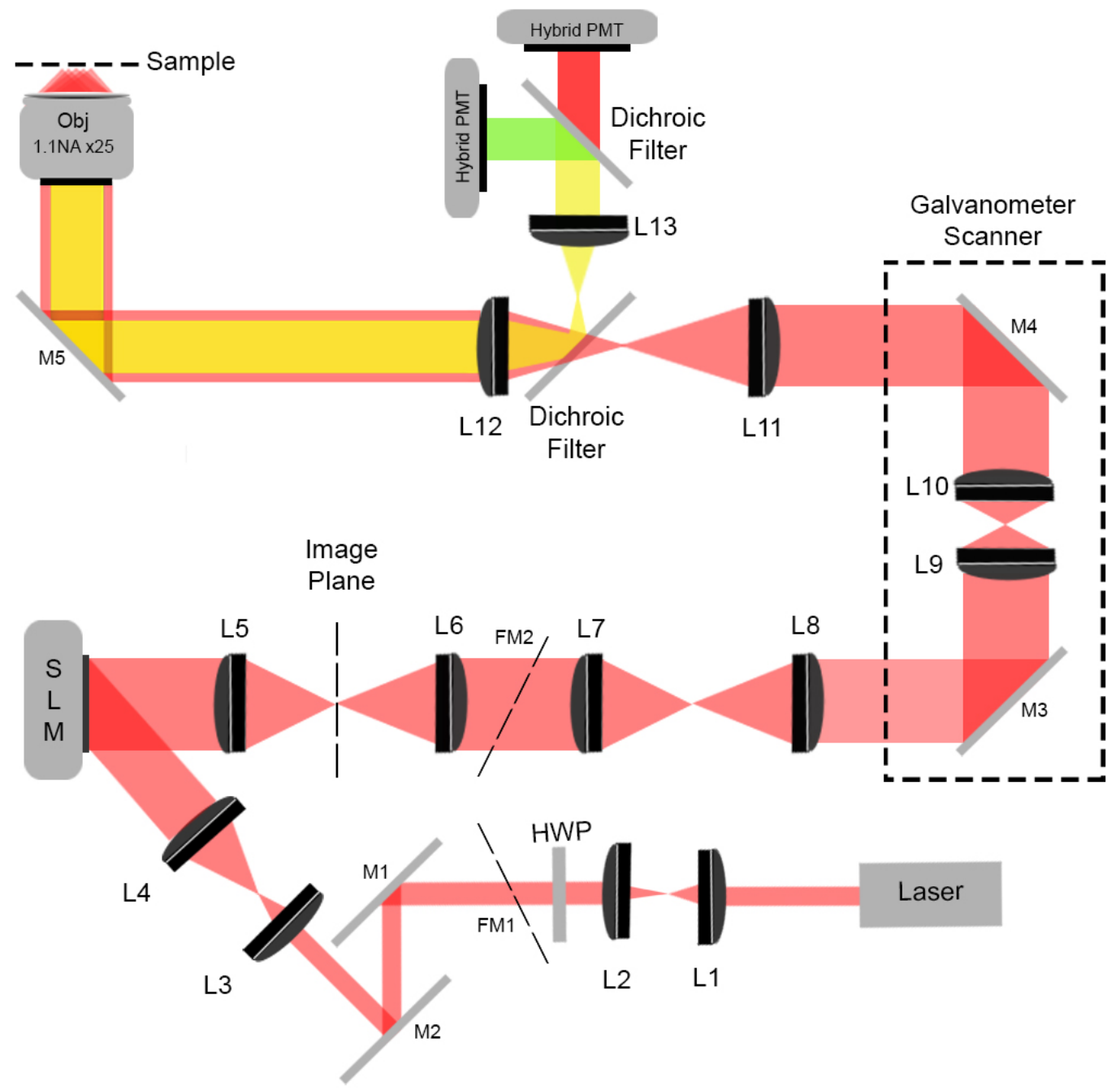

Figure 54: Pupil-Segmentation Setup. A TPM laser source expanded to overfill the SLM located in the Hologram plane, responsible for the optimization routine. The non-relevant orders are filtered out in image plane. The first order is then relayed through Galvanometer scanner. The excitation is focused and subsequent fluorescence captured by a x25 1.1 NA objective and seperated from the NIR/IR excitation by a dichroric filter. Sample fluorescence is the spectrally split onto a pair of Hyprid PMT detectors. Lenses and mirrors denoted as $L_{x}$ and $M_{x}$. 


\subsection{SLM CALIBRATION}

The Holoeye is an 8-bit device and produces phase values from 0-255. Gamma curve calibration was performed using the checkerboard pattern approach, described in section 10.3. As the Holoeye has an $86 \%$ fill factor, the zeroth order central minimum is not reduced to zero at $\pi$ (Figure 20). In order to achieve accurate gamma curve calibration the $1^{\text {st }}$ diffracting was used, which shows a maximum intensity at $\theta=\pi$ (Hermerschmidt et al., 2007). Zero value phase squares are incrementally increased while registering the intensity with a CMOS camera [Thorlabs] in the Fourier plane of the SLM. A calibrated gamma curve (Figure 55) permits accurate phase projection from $0-2 \pi$.

Holoeye NIR 920nm Gamma curve using the checkerboard approach

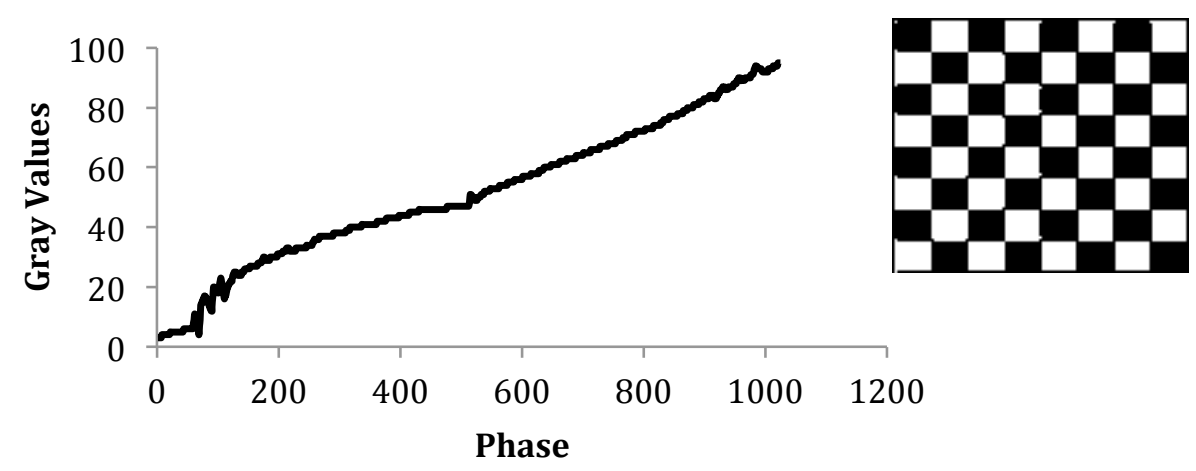

Figure 55: Checkerboard calibration of the Holoeye NIR2 and gamma curve for 920 $\mathrm{nm}$. 


\subsection{PUPIL-SEGMENTATION DESCRIPTION}

Developing the wavefront reconstruction method was the first step performed towards constructing the system employing adaptive optics. The two steps common to all wavefront reconstruction methods is the localization of the centroid of a given particle/lenslet and the subsequent calculation of the displacement gradient. Centroiding was performed using the standard LabVIEW algorithm in agreement with equation 25 and the gradient calculation, $\delta y$ and $\delta x$, using equation 26.

Accurate reconstruction and representation of wavefronts ensure a robust method of correcting phase aberrations. The number of Zernike polynomials which can be accurately reconstructed show a linear dependency with the amount of lenslets/subregions ( $N_{s}^{2}$ for a square pattern) used in a SHWFS (Porter et al., 2006).

$$
\lim _{N_{S}^{2} \rightarrow \infty} R M S=0 \quad 51
$$

Segmentation results in illuminating only a fraction of the back aperture of the imaging objective, leading to an elongated beamlet focus (Milkie et al., 2011). The large focus reduces intensity, more noticeable in TPM. To improve SNR, segmentation is accompanied by a significant increase in laser power $(x 10)$ and pixel integration time (Ji et al., 2012). The reduced illumination area $\left(\frac{A}{N_{S}^{2}}\right)$ on the imaging objective pupil limits the total number of 'independent' segments. In the present system $N_{S}=3$ which represents 9 independent segments. As 9 independent regions are insufficient to fully represent the aberrations expected, a stepped overlapping mask approach for the correction was developed. The overlapping approach (Figure 56) consists of masking an area $\frac{1}{9}$ of the pupil, which is translated vertically and horizontally by steps equal to half the width of the non-masked region, ( $\frac{1}{18}$ of the total hologram length) vertically and horizontally, providing a total of 25 regions. The relation between $N_{S}$ and the total number of subregions projected, $N_{T P}$, generated using this method is given by:

$$
N_{T P}=4 N_{S}^{2}-4 N_{S}+1 \quad 52
$$


Overlapping regions provide intermediate local phase contributions (calculated via centroid displacement). The optical surface is reconstructed on a sectioned basis for which the overlapping regions have equal contributions. Overlapping zones reduce the biased of modal fitting towards the periphery of the pupil (Espinosa et al., 2010). The final number of contributing regions generated post-overlapping $\left(N_{T O}\right)$ is given by:

$$
N_{T O}=4 N_{S}^{2} \quad 53
$$

Vertical and horizontal transitions of the mask generate 4 equal overlapping contributions per region in the centre and 2 in the border (excluding corners) (Figure 57).
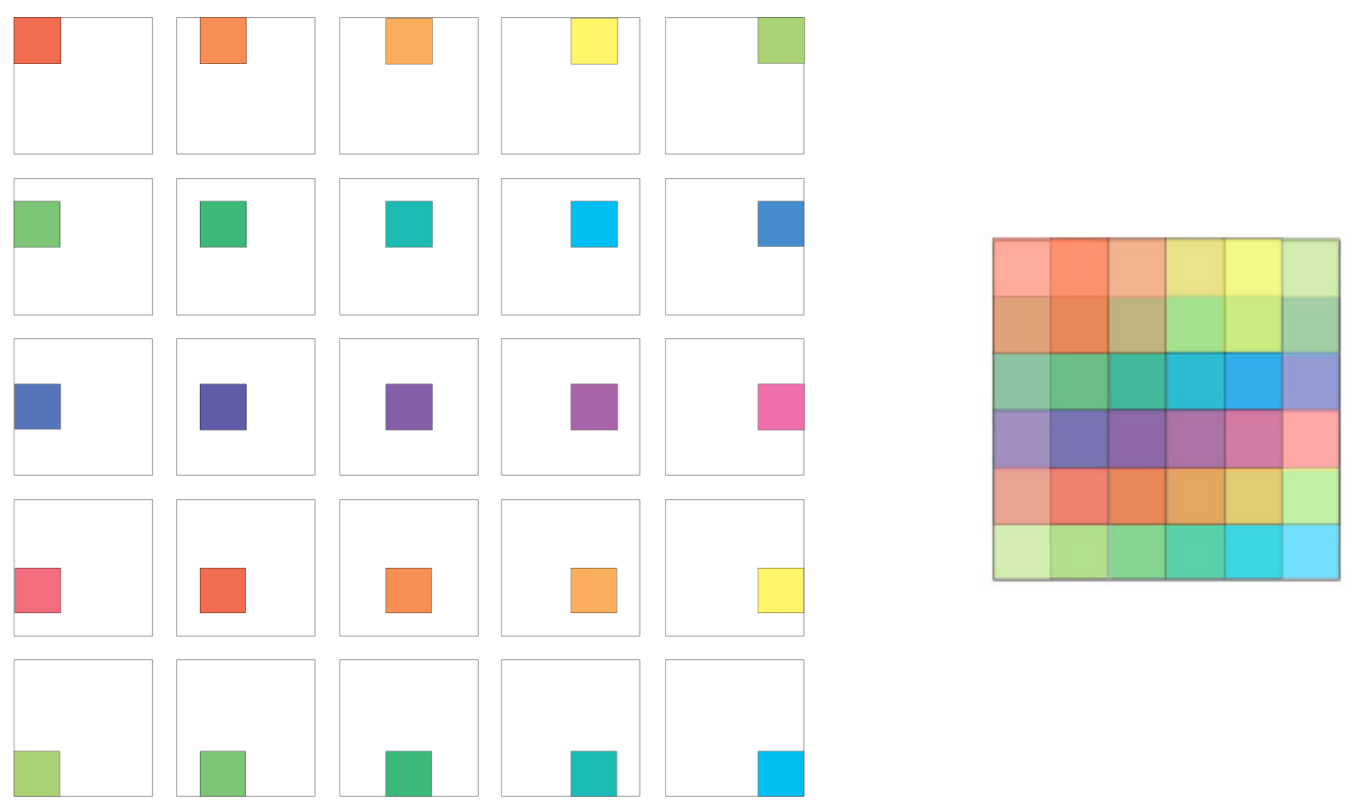

Figure 56: Graphical illustration of the stepped overlapping procedure. The black border represents the total pupil. Each square, which represents $1 / 9$ of the pupil area, is colour coded. Stepping the sections over the entire pupil space generates a final overlapped mask in which each colour coded segment contributes equally (right). For illustration purposes, a transparency was set in all the individual regions which when recombined illustrate higher influence depending on the density. 


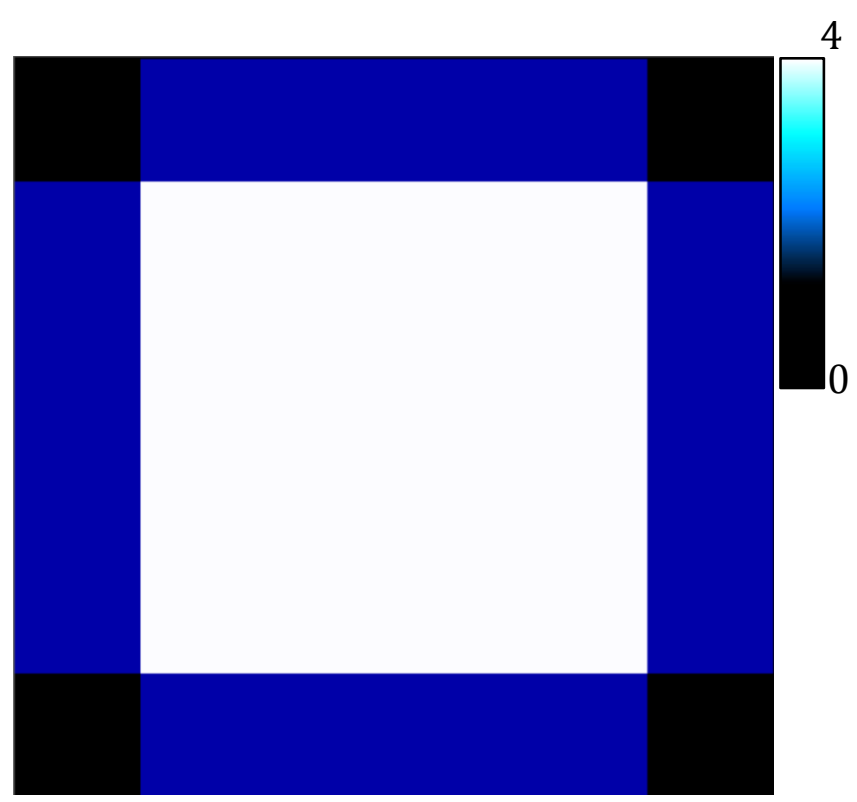

Figure 57: Overlapping regional contributions of the displacement calculation. The stepping procedure generates 4 contributing slopes for the central region (white squares), 2 for the borders excluding the corners (blue) and 1 slope for the corners (black). 


\section{RESULTS}

\subsection{SIMULATIONS}

In order to reconstruct aberrations in the image plane, a far-field simulation was performed. The 2D Fourier transform of various Zernike polynomials, which simulate an aberrated wavefront, undergoes a simulated pupil-segmentation. The Fourier transform $(\mathcal{F})$ relation between the pupil-plane of the objective $(S L M)$ and the image plane (focus), represented by the complex fields $u_{H}$ and $u_{T}$ respectively is given by:

$$
u_{T}\left(x_{T,} y_{T}\right)=\mathcal{F}\left\{u_{H}\left(x_{H} y_{H}\right)\right\} 54
$$

The phase at the hologram plane $\left(\varphi_{H}\right)$, producing lateral shifts $\Delta x, \Delta y$ in the position of the focused spot is given by

$$
\varphi_{H}\left(x_{H} y_{H}\right)=a\left(\Delta x x_{H}+\Delta y y_{H}\right) \quad 55
$$

In which $a$ is dependant on imaging characteristics and wavelength, for simulation purposes $a=1$ (Leach et al., 2006). The positional variation in the Fourier plane is calculated using centre-of-mass which provides the respective gradient (Chapter 1, Section 7.5). Once the slope data is acquired, the average slope within each postoverlapping contributing region of $N_{T O}$ is calculated, reconstructed using a zonal reconstruction (Appendix $A$ ) and subsequently fitted using a Levenberg-Marquardt routine, producing the appropriate Zernike coefficients (Figure 58) (Altenbach, Hubble Lab; 2010). This approach seeks to computationally validate the reconstruction method for randomized aberrations via the RMS. The RMS between each representation and the original wavefront was taken after normalizing to the peak-to-peak of the simulated wavefront (Ji et al., 2010). Figure 59 is the result of the phase reconstruction of an unknown wavefront (original wavefront) using various $N_{S} \times N_{S}$ segments. Zernike polynomials that do not contribute to the axial PSF (piston, tip, tilt and defocus) (Dai, 2008) have been excluded to produce the original wavefront however have been included in the reconstruction to evaluate 
the azimuthal frequency coupling between the primary and higher order aberration (i.e. defocus and spherical aberration). RMS variation is represented in Figure 60.

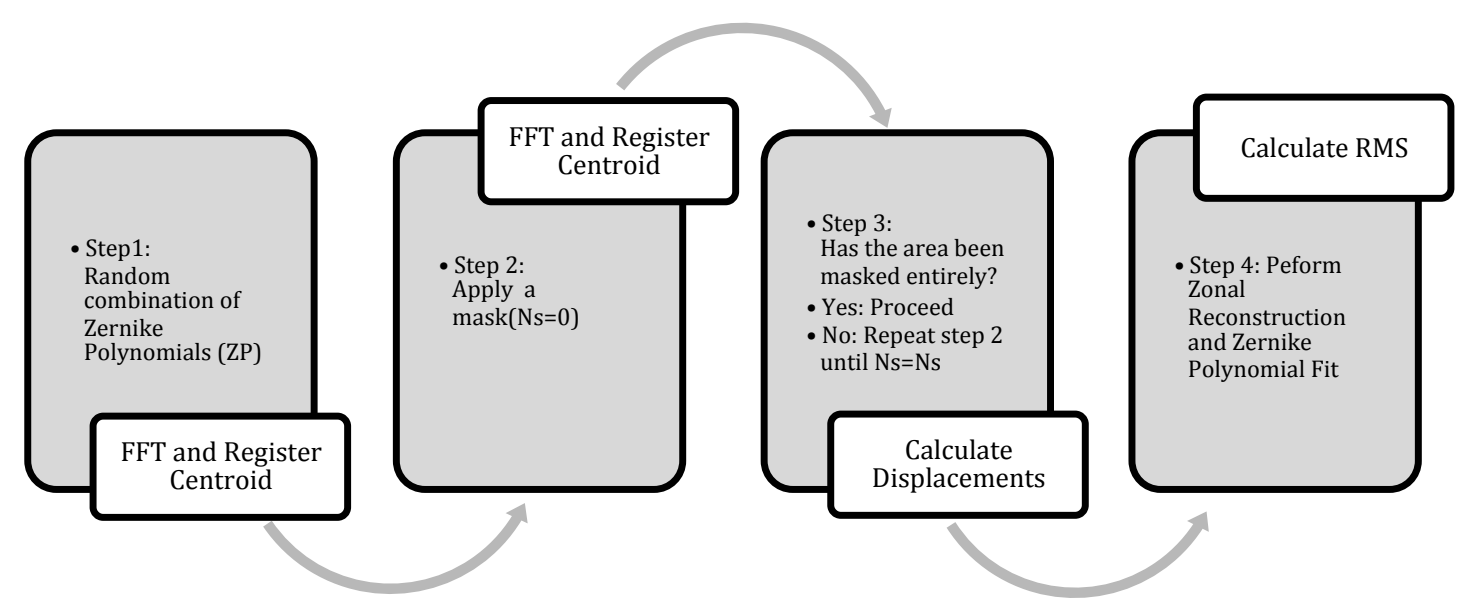

Figure 58: Flow chart for far-field simulation developed to validate pupilsegmentation wavefront reconstruction via the RMS. 


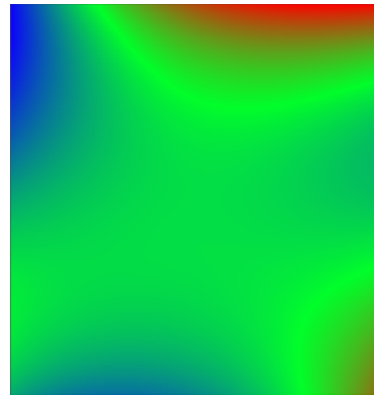

Original Wavefront
$11.6 \pi$

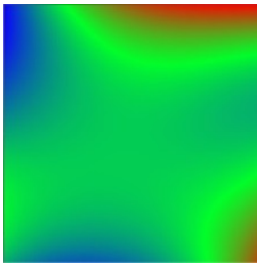

$3 \times 3+$ overlap

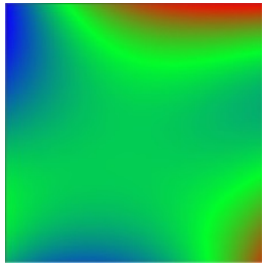

$8 \times 8$
$2 \times 2$

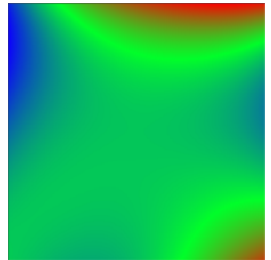

$4 \times 4$

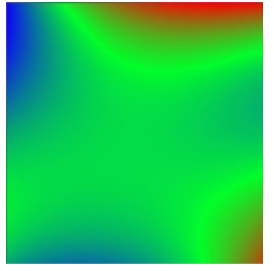

$10 \times 10$

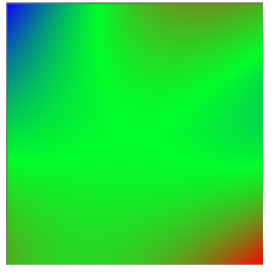

$3 \times 3$

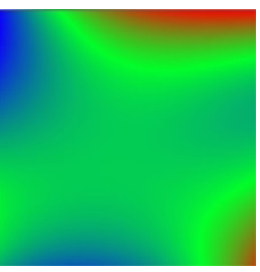

$5 \times 5$

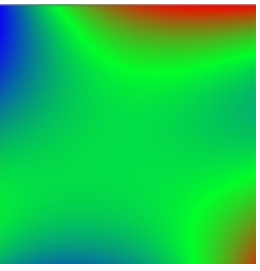

$25 \times 25$

Figure 59: Wavefront reconstruction and Zernike Polynomials fit of a complex wavefront (Original Wavefront - left) to various $N_{S} \times N_{S}$ independent or overlapping areas (right).

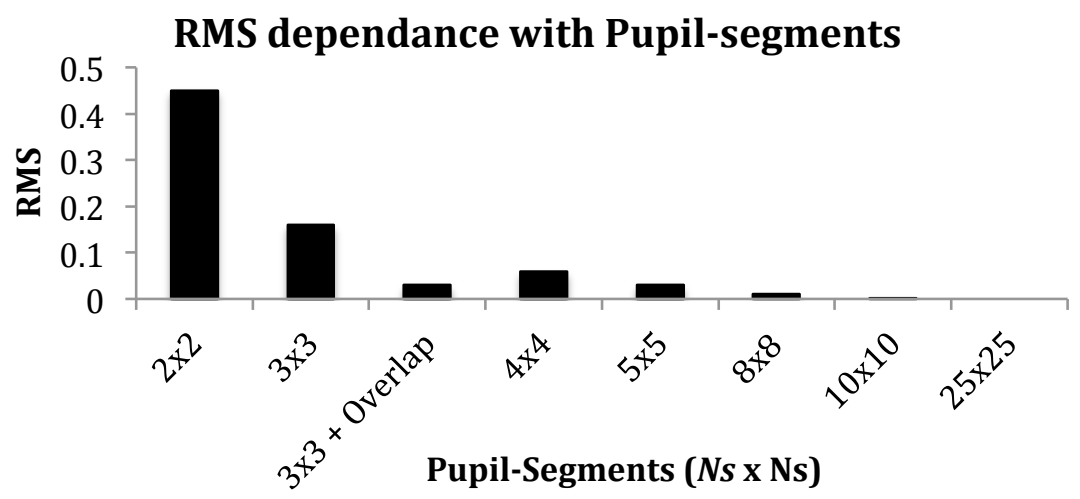

Figure 60 RMS variation with the various $N_{S} \times N_{S}$ Pupil-segmentation wavefront reconstructions.

As expected, an increase in the number of regions provides higher accuracy reducing the RMS (Eq. 51). Higher segmentation such as 64 independent regions 
(and above) is capable of fully distinguishing aberrations with the same azimuthal frequency (RMS $\leq 0.001)$. The $3 \times 3$ stepped overlap procedure and individual $5 \times 5$ segments produce equal RMS (RMS $=0.03)$. Zernike Polynomial coefficient comparison between $3 \times 3$ stepped overlap and $5 \times 5$ is shown in Figure 61 .

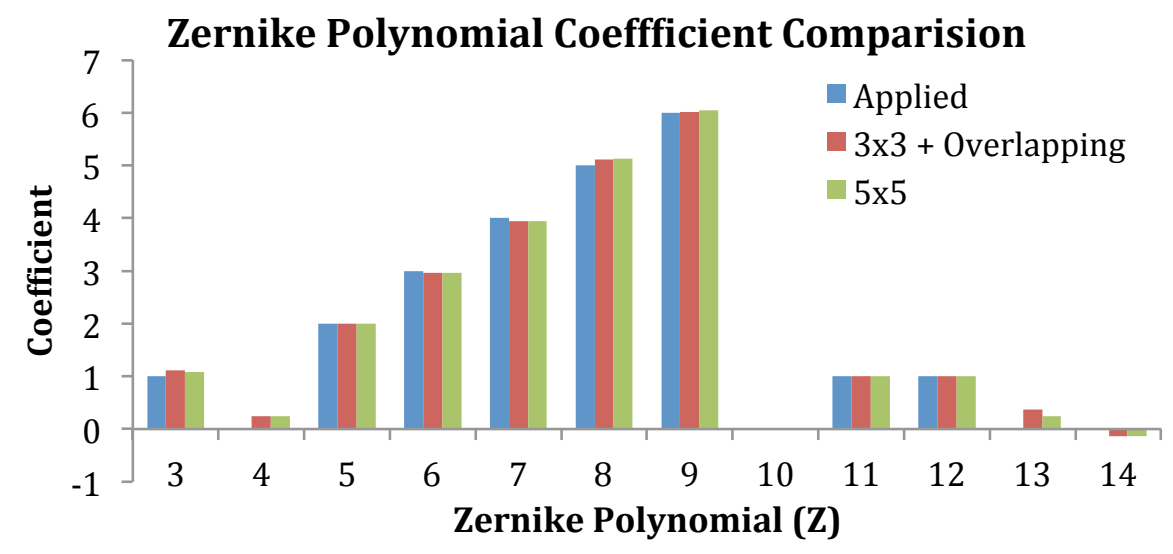

Figure 61: Zernike Polynomial coefficient comparison between $3 \times 3$ stepped overlap and $5 \times 5$ phase reconstruction. Defocus (Z4) was not applied but the reconstruction detects minor coefficient amplitude, consequence of spherical aberration (Z12) coupling.

Defocus (Z4) was not applied but the reconstruction detects minor coefficient amplitude, consequence of spherical aberration (Z12) coupling. Minor differences show that the 25 images necessary for phase reconstruction using the stepped overlap are comparable to 25 independent individual regions for $Z \leq 14$. 


\subsection{SYSTEM CORRECTION}

In order to correct for system-induced aberrations, a fixed $1 \mu \mathrm{m}$ diameter fluorescent microsphere sample [Invitrogen] underwent a 3×3-overlapped pupilsegmentation. A bead located in the centre of the FOV provides the correction for the Holoeye NIR2 SLM microdisplay flatness variation, in addition to any other aberrations generated via the optical system (i.e. misalignments). Figure 62 shows an x1.4 increase in signal and a lateral and axial measurement of $1.1 \pm 0.1 \mu \mathrm{m}$ and $2.93 \pm 0.17$ respectively. The images of the bead acquired prior AO correction are elliptical (Figure 62, top), a characteristic of astigmatism, inherent to SLM microdisplays (Bowman et al., 2010). Uncorrected beads exhibit a y-axis FWHM measurement of $1.65 \pm 0.2 \mu \mathrm{m}$ and axial FWHM of $4.01 \pm 0.21 \mu \mathrm{m}$. Normalized optical measurements using system adaptive optical correction shows reduction of the FHWM in both lateral and axial directions (Figure 64). Zernike polynomial coefficients calculated are shown in Figure 65.

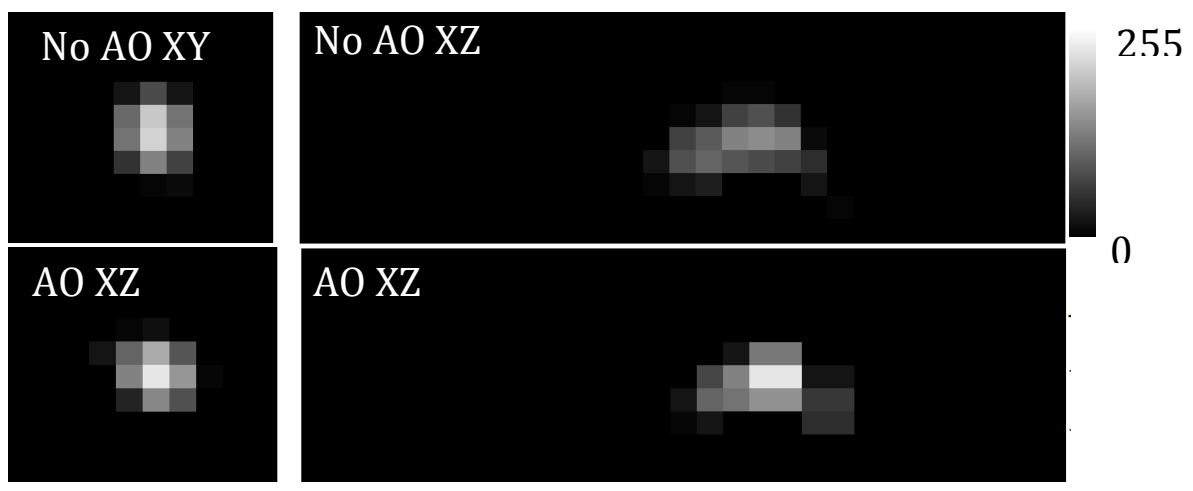

Figure 62: Original (top left) and system correction (bottom left) lateral projections of a $1 \mu \mathrm{m}$ bead projections with the respective axial projections in XZ. Contrast enhanced to aid visualization. 

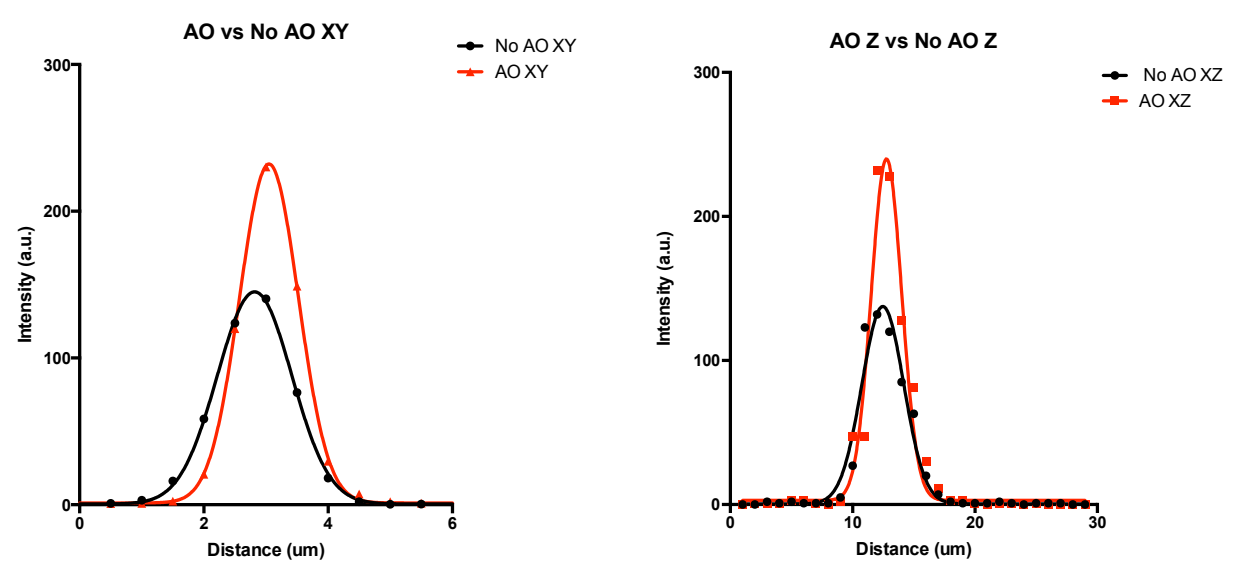

Figure 63: Comparative lateral (left) and axial (right) profile of a $1 \mu \mathrm{m}$ bead on a fixed coverslip before (black line) and after adaptive optical correction (red). Lateral measurement before and after correction is $1.65 \pm 0.02 \mu \mathrm{m}$ and $1.1 \pm 0.1 \mu \mathrm{m}$ and axial measurement is $4.01 \pm 0.21 \mu \mathrm{m}$ and $2.93 \pm 0.17 \mu \mathrm{m}$, respectively.
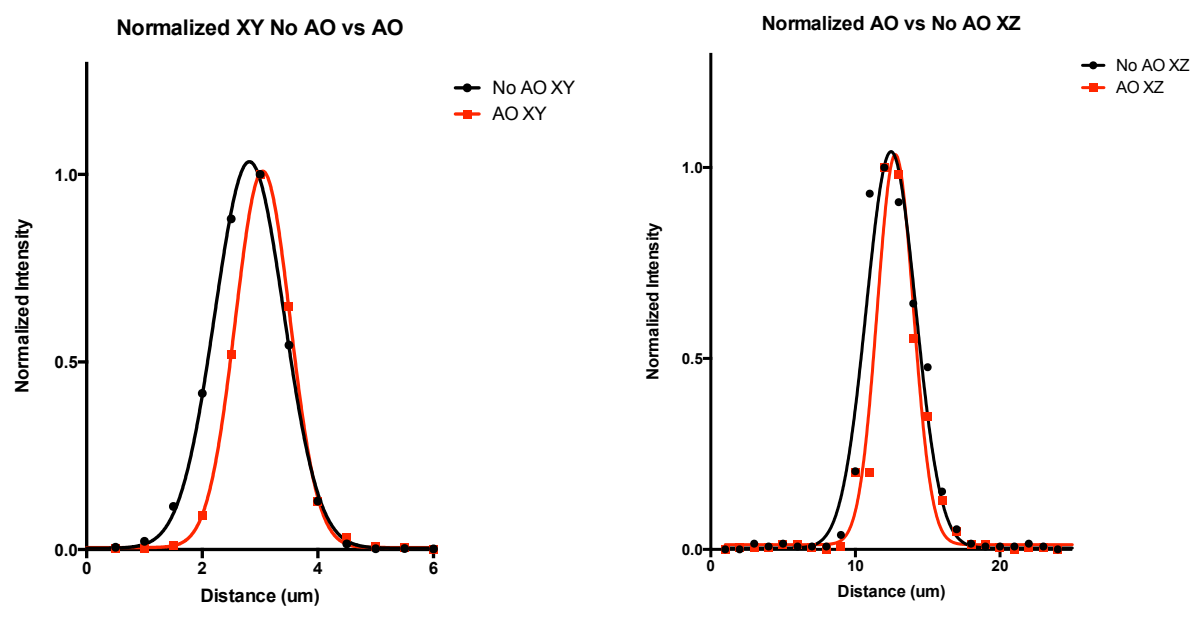

Figure 64: FWHM reduction of a $1 \mu \mathrm{m}$ due to adaptive optical correction 


\section{Zernike Polynomial Coefficients Projected for System \\ Correction}

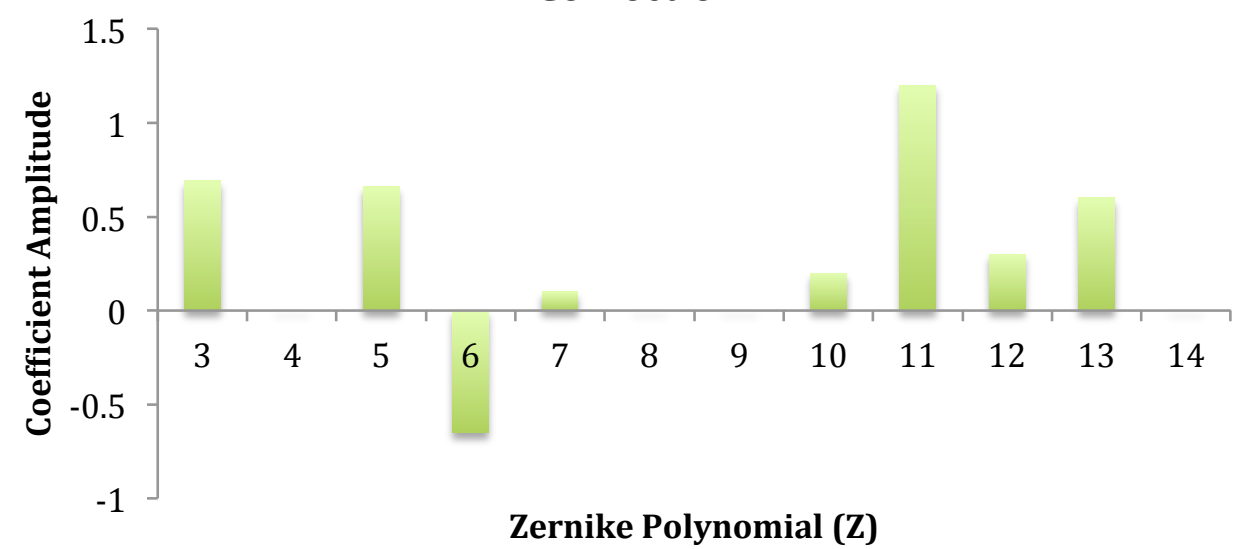

Figure 65: Projected Zernike Polynomials for system correction. Tip (Z1), tilt (Z2) and defocus (Z4) were calculated in the coefficient analysis but removed in the the reconstruction

The Zernike coefficients (Figure 65) correspond to system aberrations and are utilized with the initial blazing function. Applying the system corrections to a Convallaria Majalis sample is shown in Figure 66. A minor improvement is achieved as demonstrated by the diagonal line profile (Figure 67).
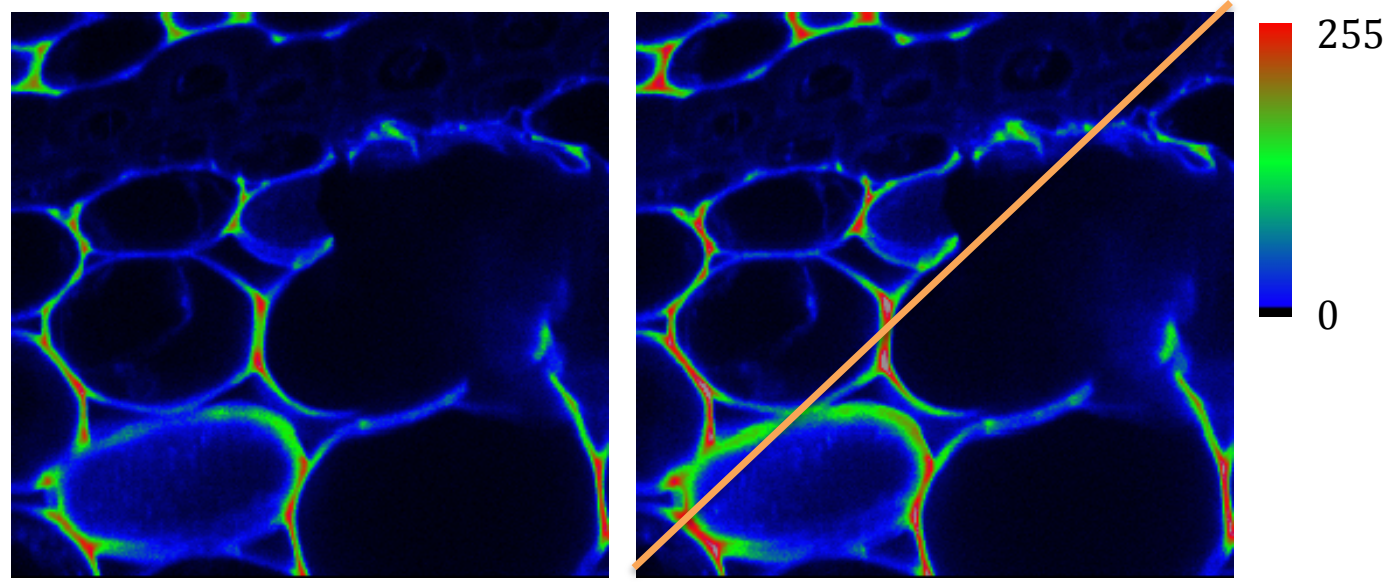

Figure 66: System correction applied to Convallaria Majalis (excitation $800 \mathrm{~nm}$ ).

Diagonal line profile in (orange) demonstrated in Figure 67. 


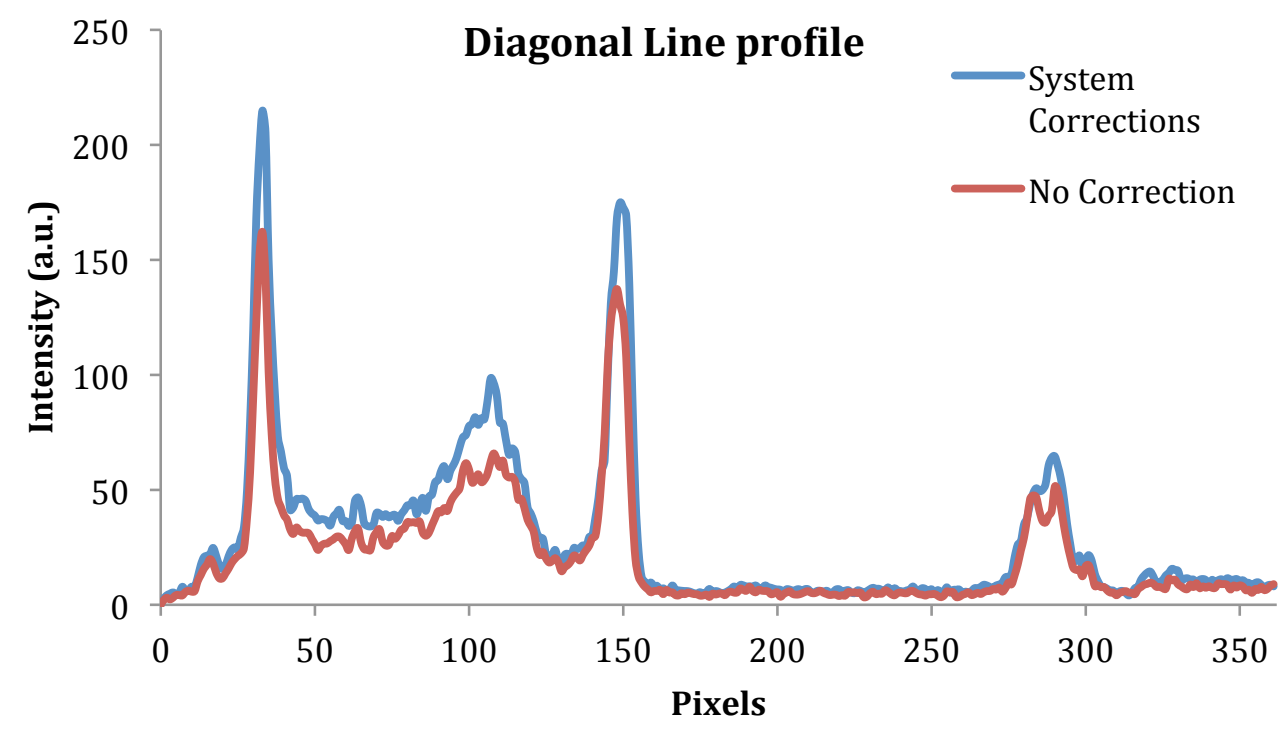

Figure 67: Diagonal line profile of Convallaria Majalis (Figure 66) with system corrections shows minor improvements.

In comparison to the fluorescent microspheres, the systems corrections once applied to the Convallaria Majalis sample demonstrate a reduced improvement. Sample refractive indexes (i.e. cellulose) introduce additional aberrations which are not corrected for by the system Zernike coefficients. Despite the fact that elimination of system aberrations improves SNR, additional correction is necessary to fully correct for sample aberrations.

\section{SAMPLE ABERRATION CORRECTION}

The developed pupil-segmentation algorithm was subsequently tested in a sample with higher inherent aberrations. It consisted of a oncological section of a Alexa-488 conjugated antibody tissue sample of approximetely $25 \mu \mathrm{m}$ thickness fixed on top of $2 \mu \mathrm{m}$ reference beads. Sample aberration correction was performed on the reference beads. The bright fluorescence signal from the beads produces an image with sufficient SNR facilitating centre-of-mass calculation during the pupilsegmentation. Figure 68 shows improvement of $x 3.5$ over the original image at a 15 $\mu \mathrm{m}$ depth using the $3 \times 3$ stepped overlapped procedure. The improvement was calculated as a ratio; the difference over the summation of the two images. 

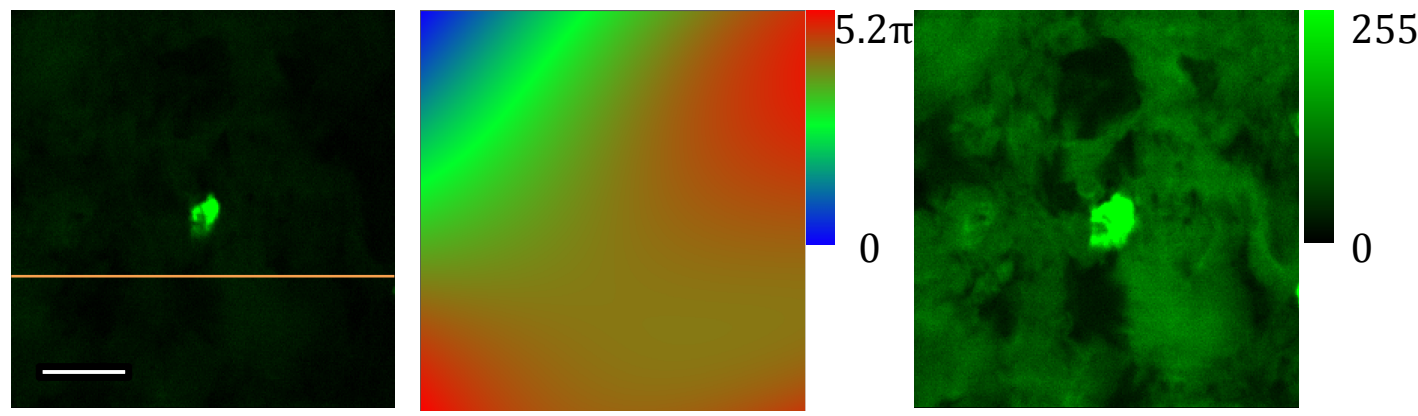

Figure 68: Sample correction before (left) and after (right) the pupil-segmentation optimization procedure. Zernike polynomial fitted wavefront applied (middle). Scale bar $30 \mu \mathrm{m}$ (white), line profile for comparative purposes $128 \mu \mathrm{m}$ (orange).

An arbitrary line profile (orange line Figure 68 common to the pre and postcorrected image is demonstrated in Figure 69.

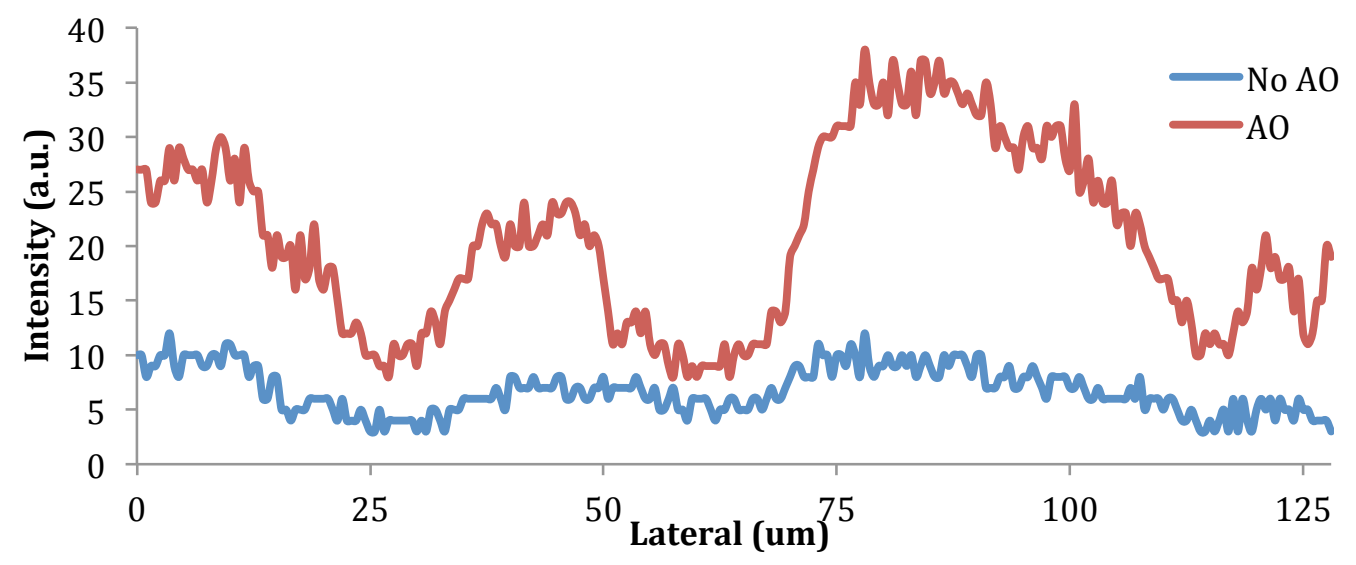

Figure 69: Comparative intensity line profile (orange line) before and after sample correction.

The coefficients for the first 15 Zernike polynomials determined in the final reconstructed wavefront (Figure 68 middle) is shown in Figure 70. Coefficient comparison between uncorrected and corrected images is shown in Figure 71. 


\section{Zernike polynomial coefficients determined for tissue sample}

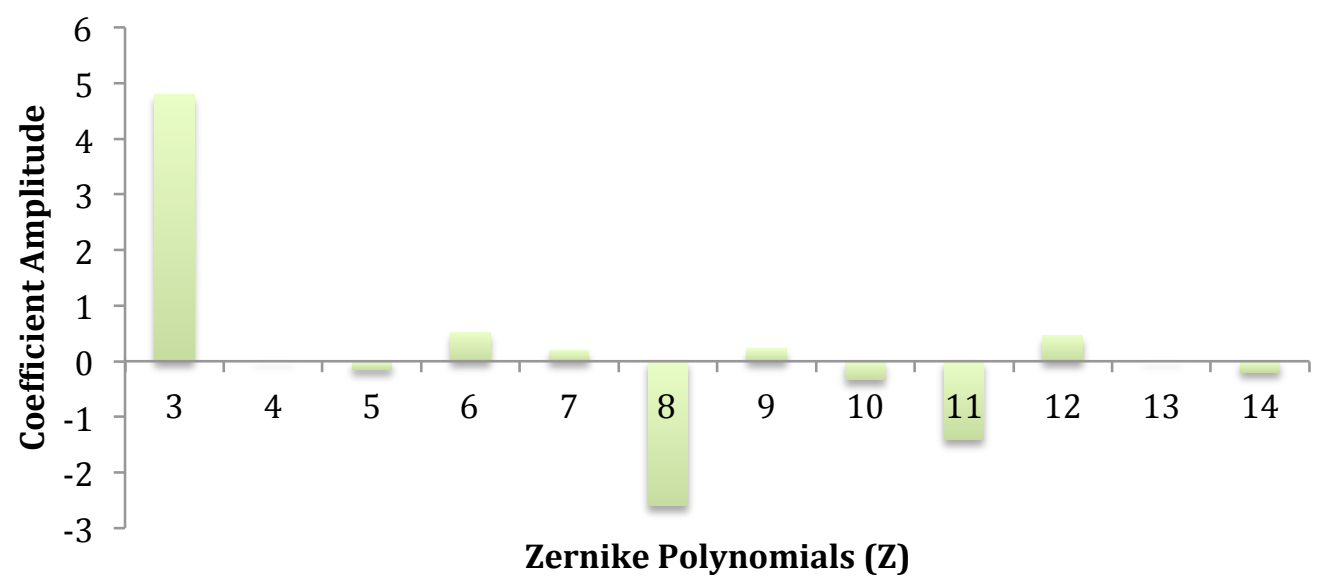

Figure 70: Sample Zernike polynomials coefficients calculated from the $3 \times 3$ stepped overlap procedure. Tip (Z1), tilt (Z2) and defocus (Z4) were calculated in the coefficient analysis but removed in the reconstruction.

\section{Projected System and Sample Zernike Polynomial Coefficient Comparision}

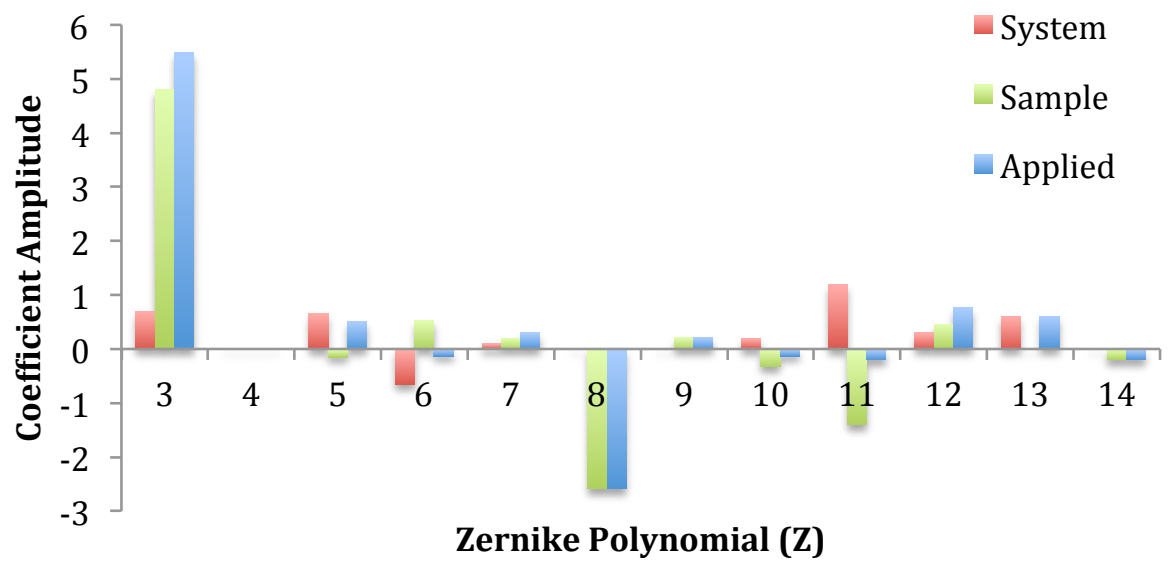

Figure 71: Comparison between coefficients projected for the system and sample Zernike polynomials. Tip (Z1), tilt (Z2) were removed and defocus (Z4) set to zero as these do not contribute to the axial PSF improvement.

The Zernike polynomial comparison between the coefficients of the corrected and uncorrected representation is shown in Figure 71. The applied values calculated as the sample coefficients added to the system coefficients represent the true Zernike 
coefficients projected on to the sample. Demonstrated is a significant influence of astigmatism (Z3), coma (Z8) and spherical aberration (Z12). In comparison, system aberrations Z6, Z10 and Z11 are reduced. Figure 71 shows that for highly aberrated samples, system correction has a reduced contribution towards the applied corrective pattern.

\subsection{FLIM WITH ADAPTIVE OPTICS}

The improvement in emission (Figure 69) was used to produce before and after adaptive correction lifetime acquisitions. In order to achieve sufficient photon counts per pixel, Figure 72 was acquired over 300 seconds. Analysis of the fluorescence transients was performed using a Levenberg-Marquardt in Tri2 with $3 \times 3$ circular pixel binning. Fitting to a single exponential decay, yielded an average fluorescence lifetime of the Alexa-488 labelled sample of $1.46 \pm 0.16$ ns in this environment (Figure 72) (McGinty et al., 2008). Figure 73 demonstrates an improved accuracy in determining the lifetime distribution.
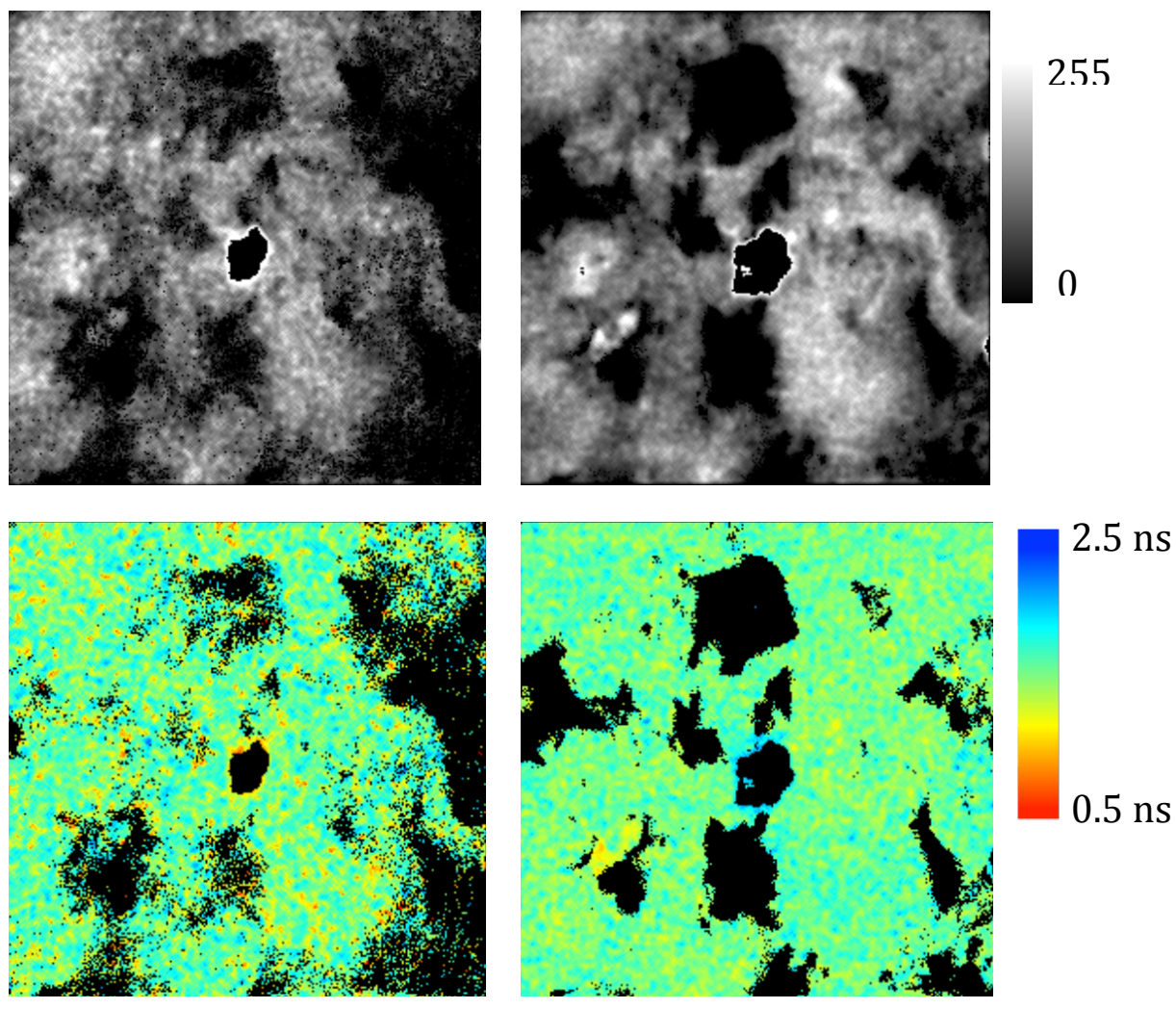

Figure 72: Intensity and lifetime distribution within the FOV before (left column) and after adaptive optical correction (right column). 


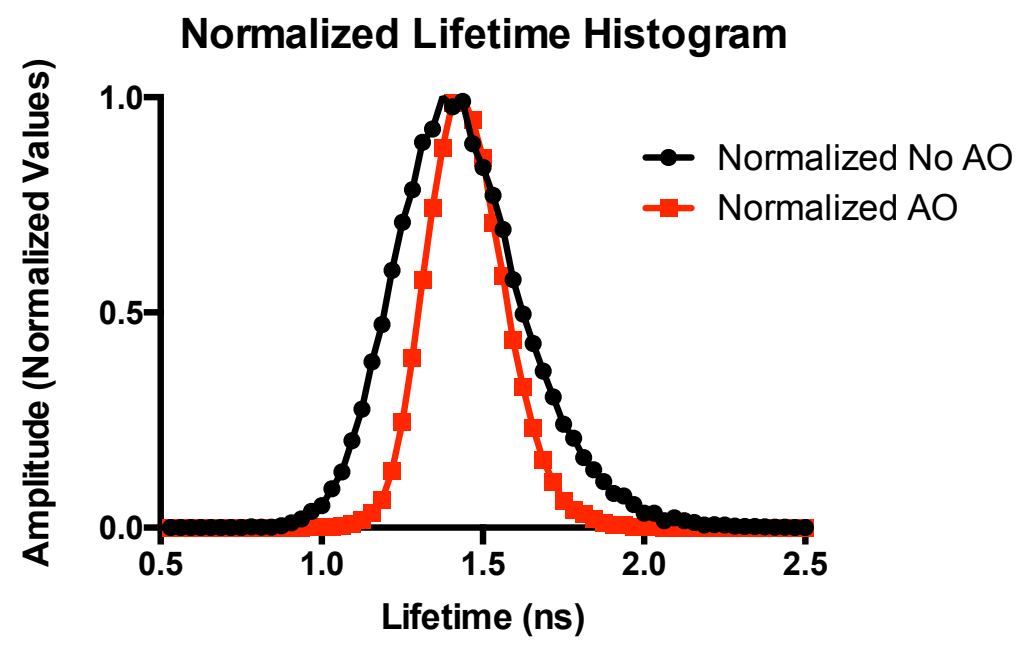

Figure 73: Normalized lifetime distribution.

The dense structure of the tissue sample produced sufficiently high distortions to visualize a noteworthy signal improvement and a narrowing of the lifetime histogram (Figure 73). Reduction of the histogram width demonstrates improved accuracy in determining the fluorescent lifetime, in thick tissue sections.

\subsection{ADAPTIVE OPTICS IN VIVO}

Histological sections are an interesting test sample but in order to test the capability of the system in a challenging in vivo imaging application where it will ultimately need to be applied, adaptive optical correction was performed in a live zebrafish embryo. Adaptive optical correction via second harmonic signal was used from a fixed zebrafish. The optical imaging of Zebrafish is particularly pertinent as these have ex-utero development and optical transparency, therefore are nearly ideal for imaging although refractive index mismatches clearly still occur and therefore aberrations for high-resolution imaging will still ultimately limit image contrast in the depth of the sample. Similar to previous experiments in histological sections, fluorescent beads were placed on top of the specimen, as the symmetrical 
shape and high fluorescence brightness makes them ideal reference points for the optimization procedure (Figure 74). The aberration coefficients determined are shown in Figure 76 and comparison with the system correction (uncorrected) in Figure 77.
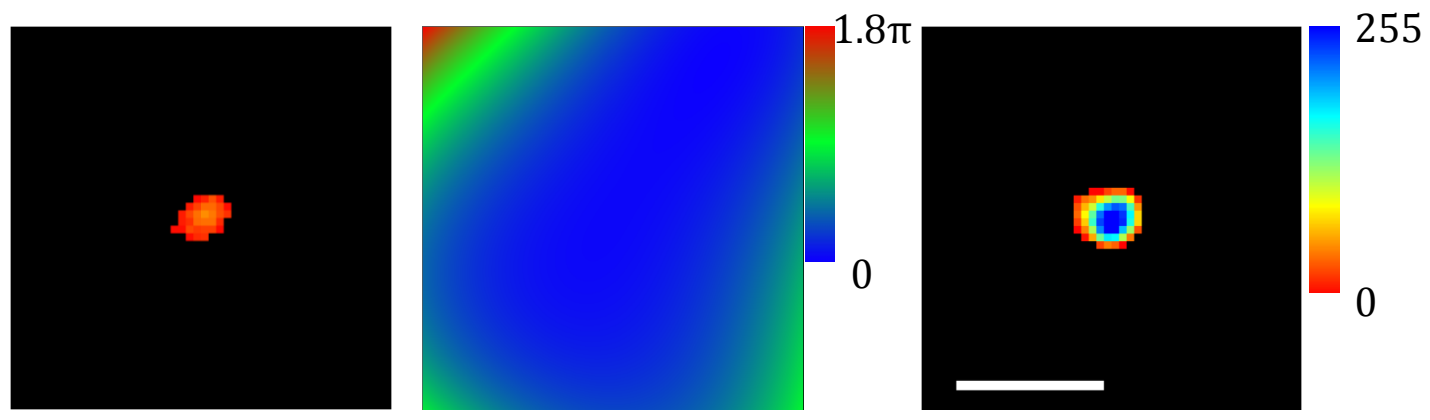

Figure 74: Intensity improvement using $2 \mu \mathrm{m}$ beads located on top a Zebrafish sample $(300 \mu \mathrm{m})$. Scale bar $10 \mu \mathrm{m}$.

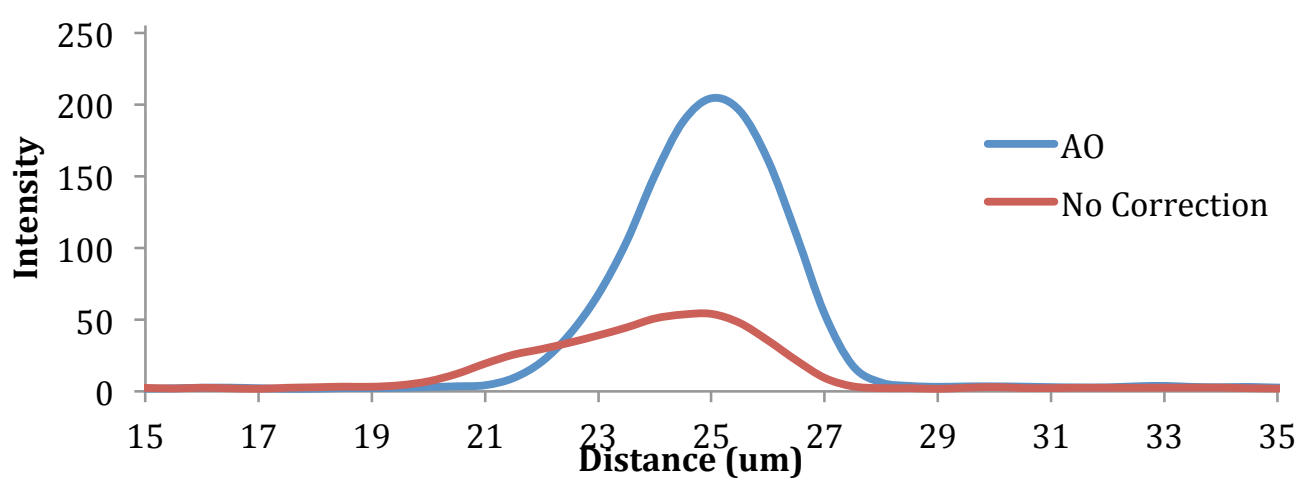

Figure 75: Lateral line profile of the bead located on top of the Zebrafish sample (Figure 74) before (red) and after (blue) AO correction. 
Sample Zernike Polynomial Coefficient Projected

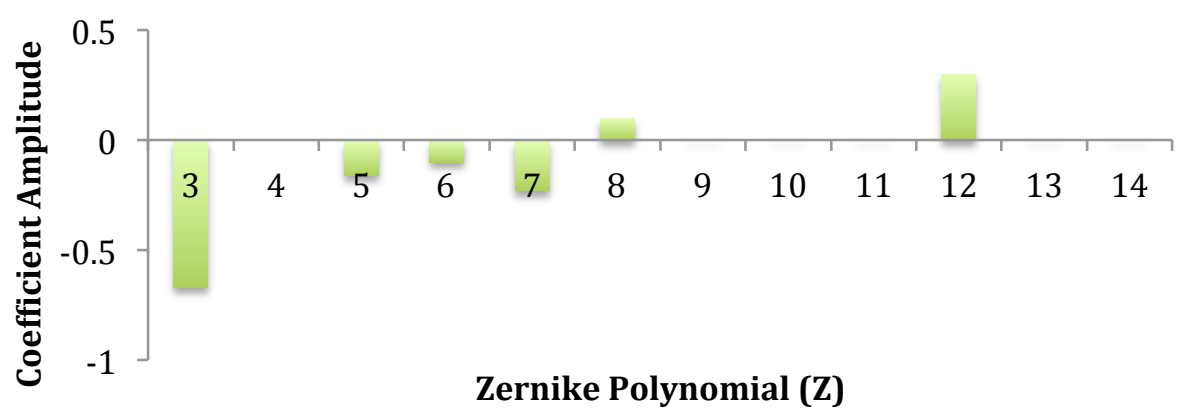

Figure 76: Zernike polynomial coefficients determined to correct for the Zebrafish distortions with respect to the reference bead. Tip (Z1), tilt (Z2) were removed and defocus (Z4) set to zero as these do not contribute to the axial PSF improvement.

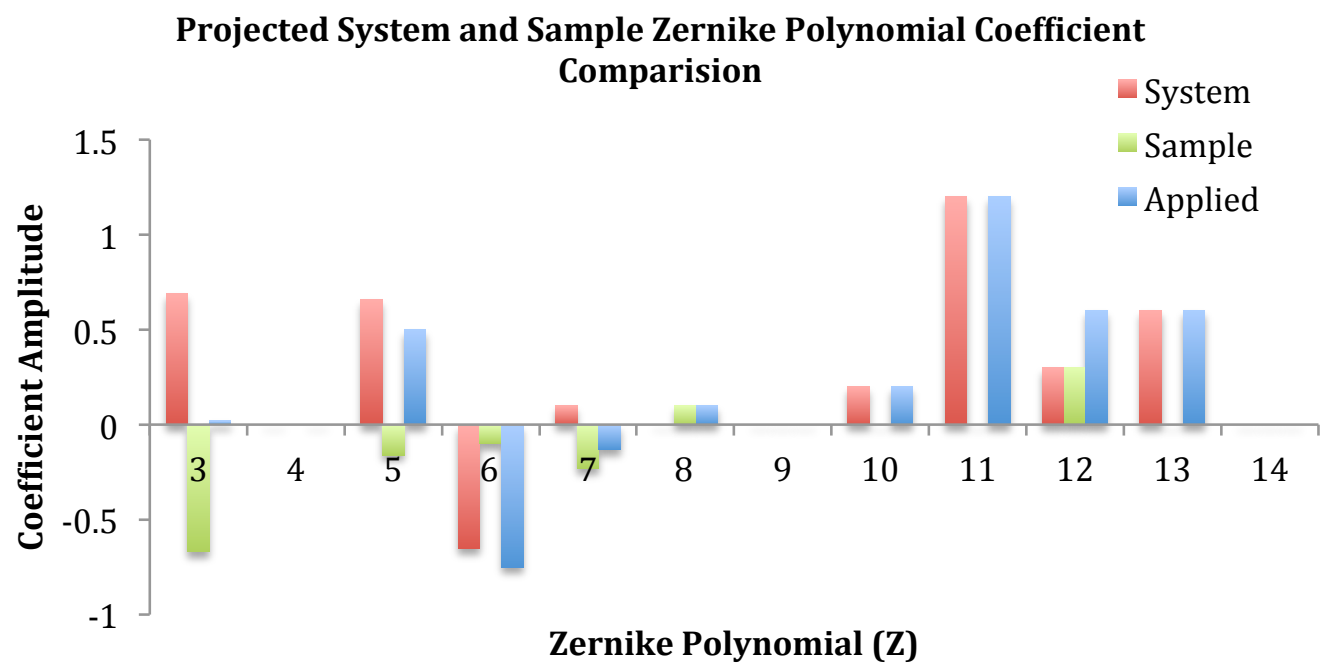

Figure 77: Comparison between coefficients projected for the system and sample Zernike polynomials for Zebrafish sample. Tip (Z1), tilt (Z2) were removed and defocus (Z4) set to zero as these do not contribute to the axial PSF improvement.

Coefficient comparison between the uncorrected and sample corrected Zernike polynomials shows the applied coefficient values (Figure 77). In agreement with previously reported zebrafish optical characterization, spherical aberration is a main contributor to resolution and SNR degradation (Bourgenot et al., 2012; Gould et al., 2012; Rueckel et al., 2006). As Zebrafish generate less aberration than the oncological sample (Figure 71), the system corrections (Z5-Z14) contributed 
positively towards the applied coefficients, with the exception of $Z 3$, which has no contribution towards final improvement. Despite significant improvement residual aberrations remain, visible in asymmetric bead profile (Figure 74, Figure 75).

Zebrafish produce substantial second-harmonic (SHG) signal (Huang et al., 2011). SHG suffers from aberrations due to turbid media (Campagnola and Loew, 2003). Applying the aberration corrections obtained with the $2 \mu \mathrm{m}$ beads improved contrast and brightness of SHG images (Figure 78).
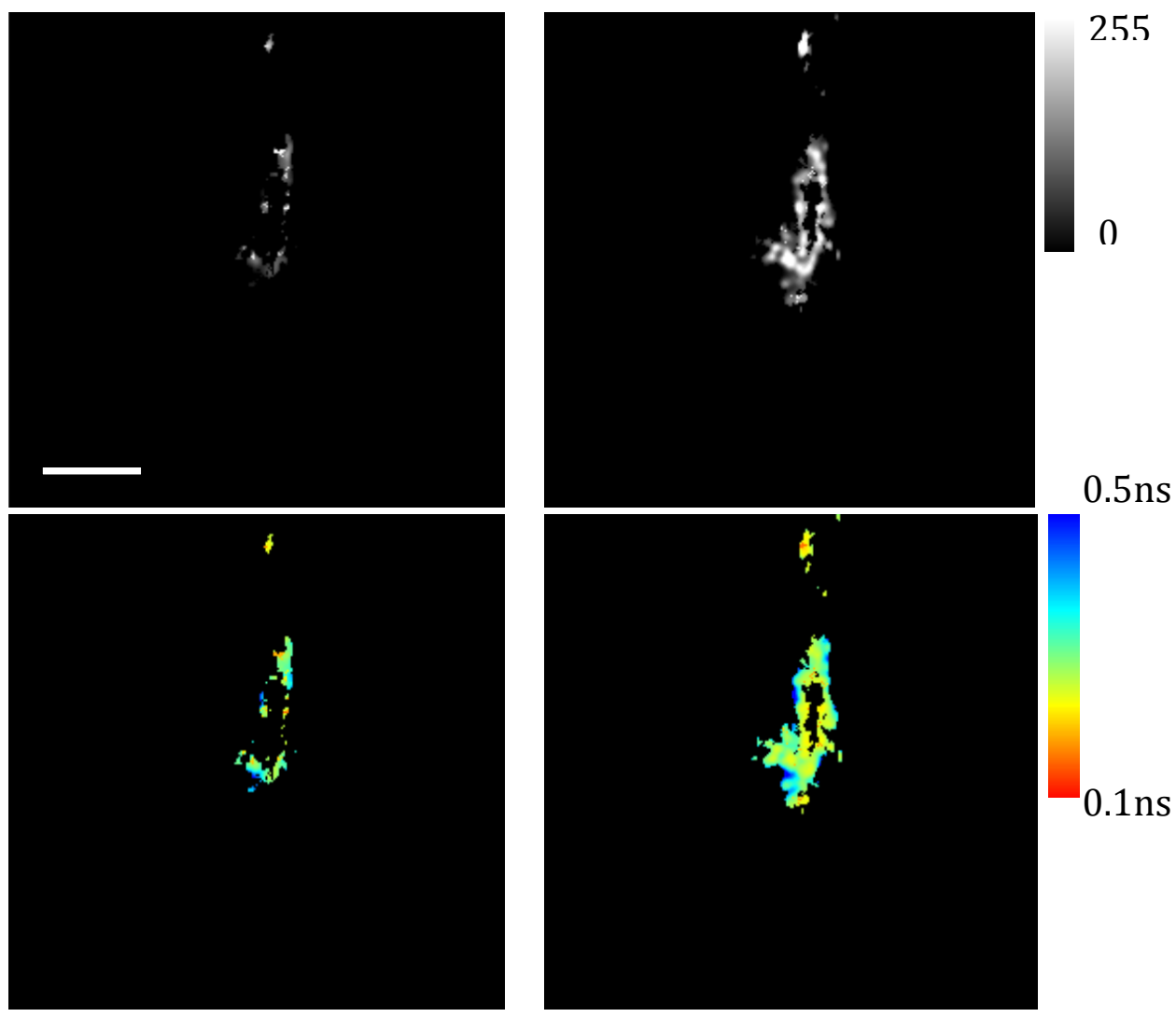

Figure 78: Zebrafish SHG Intensity and lifetime distribution before (left column) and after adaptive optical correction (right column). Scale bar $30 \mu \mathrm{m}$.

Despite residual aberrations, there is an increase in the SHG signal retrieved after the adaptive optical correction. The application of AO via SHG using a simple and intuitive approach demonstrates further potential applications in order to obtain 
high-resolution images of unstained collagen and other structures such as microtubules (Zipfel et al., 2003).

At a depth of $30 \mu \mathrm{m}$ the scan size was altered to image an entire cross section of the zebrafish tail (Figure 79). The improvement in SHG signal is significantly lower as the pupil-segmentation was performed for a region a quarter of the size. Nevertheless an improvement is visible on the right hand side of the respective corrected image and in the centre of the field where muscle structure gains increased intensity (Figure 79,Figure 80).
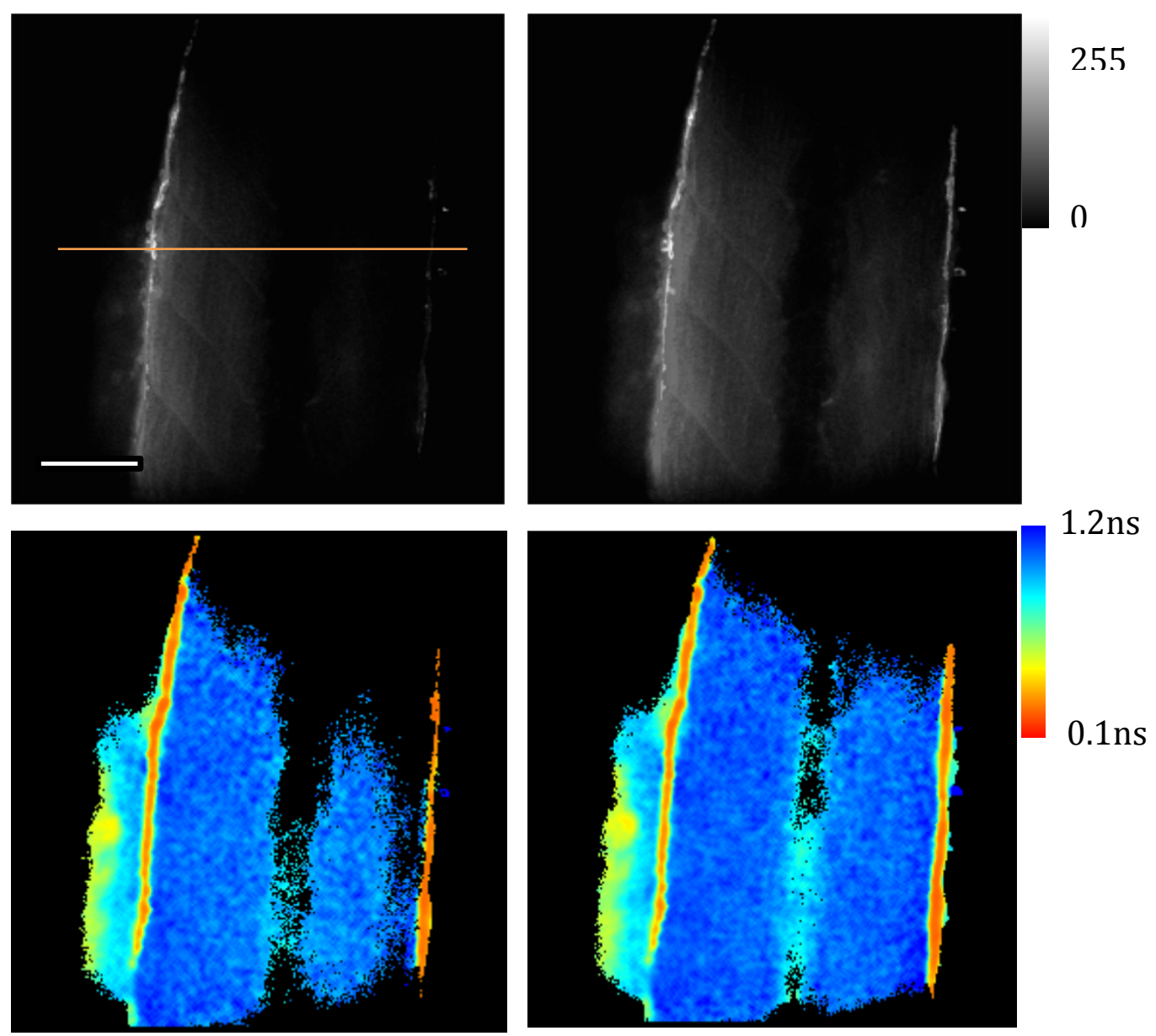

Figure 79: Optical section of Zebrafish at a $30 \mu \mathrm{m}$ depth. Intensity and lifetime distribution before (left column) and after adaptive optical correction (right column) Scale bar $100 \mu \mathrm{m}$, line profile $450 \mu \mathrm{m}$ (orange). 


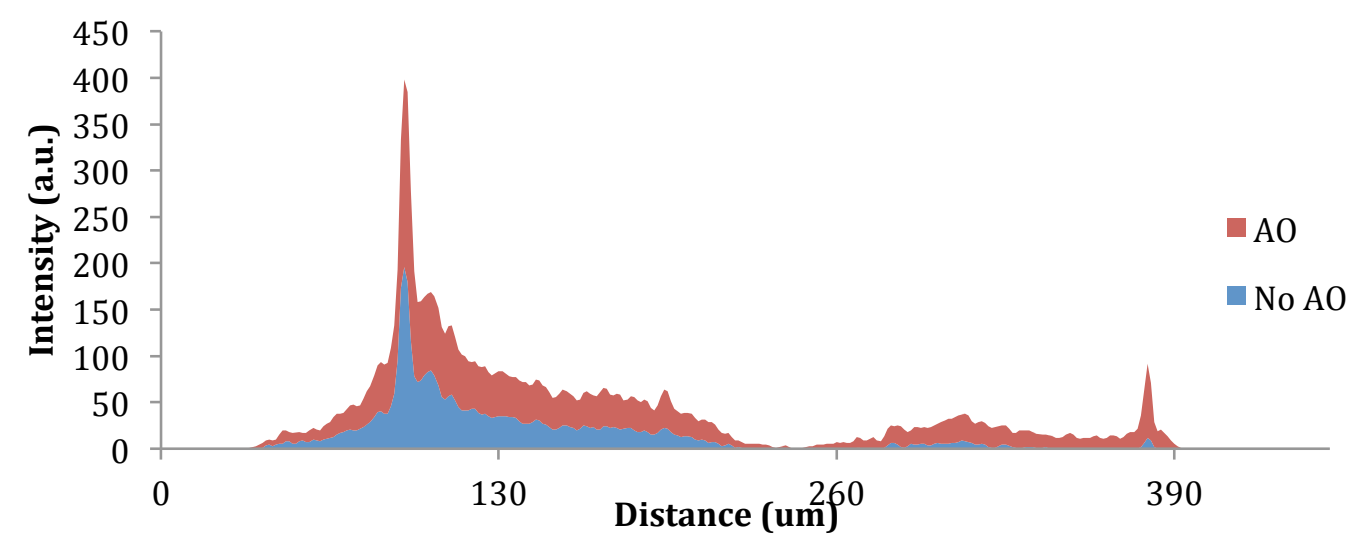

Figure 80: Comparative line profile (orange line - Figure 79) before (blue) and after (red) correction.

\section{DISCUSSION}

Imaging techniques centred on improving intensity and at-depth resolution by characterizing the aberration wavefront have been used over the last decade in a range of various applications. Typically utilizing a DMM or SLM, the wavefront corresponding to the calculated aberration is introduced in the optical pathway annulling refractive index mismatches encountered throughout the sample.

Reconstruction based on Zernike polynomials are shown to operate with higher errors once presented with a highly irregular wavefront due to smoothing that removes higher spatial frequencies (Panagopoulou and Neal, 2014). Characterization of phase aberrations generated by in vivo samples using an overlapping pupil segmentation algorithm via a zonal reconstruction (Ji et al., 2010) generally show wavefronts without abnormal segments, unlike corneal aberrations (Smolek and Klyce, 2003). Overlapping areas permit larger pupil segments providing a suitable mean of overcoming SNR limitation due to NA reduction. However, these regions cease to be fully independent. The influence of various equally contributing regions serves to average the amplitude, subsequently reducing adequate representation of higher spatial frequencies. Similar to a SHWFS, there is a trade-off between dynamic range and measurement sensitivity. In previously demonstrated 
publications, the total amount of pupil segments necessary to achieve neardiffraction/diffraction limited resolution in vivo at depth vary between 81 and 100; which are sufficient to fully characterize aberrations via Zernike polynomials (Ji et al., 2010; Ji et al., 2012; Porter et al., 2006). In fact, reference to the various aberration functions (astigmatism, spherical) is made, further enforcing the need for full characterization of the phase aberrations in optical terms. Although segmentation of the pupil is in nature a zonal approach (Milkie et al., 2011), full characterization in optical terms via a modal estimation such as Zernike polynomials, provides increased insight along with a simpler interpretation. In addition, for lower order aberrations modal reconstruction produces lower RMS than zonal estimation (Southwell, 1980). The improvements shown in this chapter, demonstrate that pupil-segmentation is not exclusively a zonal reconstruction method.

The blaze function projected on the SLM is relayed to the imaging pupil reducing the NA to 0.9, further decreased during pupil-segmentation. Segmentation of the pupil results in an elongated beam focus and intensity reduction. To increase SNR there is a significant increase in laser power (x10). Pupil-segmentation is performed by consecutive imaging while selectively masking the blazing function exposing $\frac{1}{9}$ of the original area which is translated across the pupil. The stepped overlapping procedure consists of stepping the exposed blaze function by half its own width or $\frac{1}{18}$ of the total hologram length vertically and horizontally generating intermediate slope values, improving accuracy of the reconstruction. Confirmed via simulations, the stepped overlapping procedure demonstrated comparable accuracy with the equivalent independent regions for $Z \leq 14$.

Fluorescent beads mounted on a coverslip served to determine the system-induced aberrations, principally from the SLM. System aberrations under the same optical configuration are considered constant. The comparative results demonstrated were acquired under the same conditions (power, time etc.) therefore the improvement results from the calculated phase pattern. Aberrations that do not contribute to the axial PSF have been excluded as these simply shift the image plane in $x, y$ or $z$. 
Sample aberration correction is represented as the result of the system Zernike coefficients in combination with the sample aberrations calculated. In the cases exhibiting sample correction, the absence of adaptive optical correction is equivalent to system corrections as these are incorporated in the SLM's initial imaging function. Therefore signal improvement is the consequence of suitable phase conjugation of the optical aberration function.

Fluorescence emission of a tissue sample and SHG from a Zebrafish sample demonstrated the system's capability of calculating and correcting for aberrations over a range of potential samples. Using the stepped overlapped procedure a x3.5 increase in signal at $15 \mu \mathrm{m}$ depth was obtained in a dense tissue structure, typically used in bio-medical oncological studies. The increased photon count resulted in a narrowing of the lifetime histogram (Figure 73). Zebrafish show a strong SHG signal in the tail region due to well-defined muscle. Pertinent to a large range of biomedical research, Zebrafish with fluorescent beads located on top, which served as a reference for the optimization, underwent pupil-segmentation adaptive optical correction demonstrating an x3.8 signal improvement. The optical transparency of the specimen does not induce high coefficient amplitudes. Despite the increase in SNR demonstrated, it is evident that the PSF presented in Figure 74 is not symmetrical, indicating residual aberrations. As such, in the case of minor coefficient fluctuations, increased sensitivity generates higher definition of sampleproduced distortions; therefore smaller pupil segments become necessary to achieve the full correction. 


\section{FUTURE OF THE PROJECT}

The experiments described above have been performed under uniform illumination conditions. In order to utilize the available laser power more efficiently, a motorized beam expansion accompanied by rapid translational stages can be used to direct the excitation laser to a specific segment of the SLM under investigation, therefore improving SNR. This implementation would permit smaller regions on the SLM due to higher photon flux providing increased sensitivity and higher resolution of the wavefront in a turbid media.

In the case of a pre-corrected (Hamamatsu SLM) or acceptably flat SLM (Boulder) segmentation bypassing the SLM has the potential to provide a simple and intuitive method to determine the sample-induced aberrations. A pair of mirrors on a translation stage inserted in the laser path can easily bypass the SLM therefore fully illuminating directly the imaging objective. A simple pinhole adequately mounted on xy stages would provide sequential segmentation across the imaging pupil. A pre-defined aperture along with the amount of steps produced defines the sensitivity of the reconstruction. Utilization of a range of pinhole sizes can therefore tailor the sensitivity via the illuminating segmentation area. Calculation of the wavefront in terms of circular Zernike polynomials can then be projected on the SLM, which is simply relayed, fully illuminating the back aperture of the imaging objective.

In resemblance to the system aberrations, the refractive index variation for a given depth is considered static as the sample is fixed. In the case of a live sample in which aberrations vary, it becomes possible to adequately characterise refractive index variations over time (Ji et al., 2010). Potential future experiments may include optical polynomial characterization of a variety of in vivo samples as a function of time. Investigation of signalling pathways via FRET-FLIM may be performed in vivo, such as mice containing a biosensor. 


\section{CHAPTER 4- CONCLUSION}

\section{SUMMARY}

The combination of time-correlated single photon counting and TPM is the method of choice used to accurately determine the fluorescence lifetime in fixed and in vivo samples in biological imaging programmes.

Two problems are key to improving performance of multiphoton FLIM system for in vivo applications: 1) acquisition speed and 2) improvement in imaging resolution due to diminution by optical aberrations. In this thesis both of these issues have been addressed in isolation having developed a multifocal multiphoton FLIM system and a single beam scanning FLIM pupil segmentation adaptive optics platform. Taking each system in turn:

1) Multifocal excitation array generated by a spatial light modulator selectively scans individual fractional regions of the sample. The descanned emission subsequently projected on a SPAD array permits the determination of interacting specimens approximately 30 fold faster. These developments permitted 3D visualization of cellular masses via FLIM in vivo at a rate of 10 s per acquisition

2) Adaptive optical correction was employed on a standard multiphoton system. Zernike polynomials permitted improved signal-to-noise ratio via pupilsegmentation. The spatial light modulator conjugate with the pupil of the imaging objective selectively scans the sample with a segmented beam. Improvements are demonstrated using second-harmonic generation and fluorescent lifetime.

The development of these techniques provide a valuable new approach to addressing physiologically important questions by the measurement of fluorescence lifetime changes which result from protein interactions. 


\section{FUTURE PROSPECTS}

There are a significant number of future prospects for this work, further described in each respective section. In summary, the developed hardware described in Chapter 2 may be modified for high-content screening, multi-wavelength detection, anisotropy, super-resolution such as STED, flow cytometry, FCS and single-molecule imaging. Replacing the SLM for a fixed diffractive optical element would improve light throughput excitation efficiency and the excitation may be replaced for a visible laser, in which the confocal pinholes may result from the small detection area. Enhancement in photon detection efficiency of the SPADs, higher fill factor including higher transfer rates also serves to improve the system while facilitating development.

The Adaptive Optics Chapter 3 considers a re-evaluation of the system design to accommodate the Nikon 1.1 NA objective and the incorporation of XZ stages to selectively scan the SLM improving signal-to-noise ratio of the optimization acquisitions permitting smaller pupil segments along with higher sensitivity. The incorporation of a centroiding algorithm with higher accuracy also serves to improve reproducibility. Once finalized, the purpose of the microscope will be the imaging macrophages and monocytes in vivo at improved depth with higher resolution. 


\section{CONCLUSION}

TCSPC acquisition is the most accurate method to determine Förster resonant energy transfer via fluorescence lifetime. Described throughout is the development of novel alternative methods to reduce the limitations of the typical imaging multiphoton fluorescence lifetime acquisition.

To achieve statistical accuracy, single beam TCSPC-FLIM is accomplished accompanied by a large acquisition time. In order to accelerate the acquisition without sacrificing time resolution the multifocal multiphoton FLIM system MegaFLI produces high-aperture foci, reducing higher order photodamage effects through parallelization, while maintaining superior live-cell compatibility, reduced photobleaching of non-target planes and optical sectioning. Multifocal illumination system coupled with the multi-channel TCSPC detection architecture lifts the fundamental limitation imposed by count rates in standard single-channel experiments. The system permits highly sensitive fast FLIM acquisitions in a matter of seconds, instead of minutes.

TPM combined with fluorescence lifetime imaging permits spatiotemporal monitoring of the molecular and cell biological responses with improved optical penetration. However refractive index heterogeneities induce aberrations capable of severally degrading the resolution and signal-to-noise ratio of the acquisition. Zernike polynomial based adaptive optical correction produced via an overlapping pupil-segmentation algorithm serves to reduce the influence of scattering throughout the sample, increasing lifetime determination accuracy. 
Matlab used for zonal reconstruction:

function $W=$ zonalReconstruction(Sx, Sy, ds);

$$
\begin{aligned}
& {[n, n]=\operatorname{size}(S x) ;} \\
& S=\left[\operatorname{reshape}\left(S x^{\prime}, 1, n^{*} n\right) \operatorname{reshape}\left(S y^{\prime}, 1, n^{*} n\right)\right]^{\prime} ; \\
& E=\operatorname{get} E(n) ; \\
& {[U, D, V]=\operatorname{svd}(E, 0) ;} \\
& D=\operatorname{pinv}(D) ; \\
& C=\operatorname{getC}(n) ; \\
& W=V^{*} D^{*} U^{\prime *} C^{*} S ; \\
& W=\operatorname{reshape}\left(W^{\prime}, n, n\right) . / d s ;
\end{aligned}
$$

function $E=\operatorname{get} E(n)$;

$$
\begin{aligned}
& E=\operatorname{zeros}(2 * n *(n-1), n * n) ; \\
& \text { for } i=1: n \\
& \text { for } \mathrm{j}=1:(n-1) \\
& E((i-1) *(n-1)+j,(i-1) * n+j)=-1 ; \\
& E((i-1) *(n-1)+j,(i-1) * n+j+1)=1 ; \\
& E\left((n+i-1) *(n-1)+j, i+(j-1)^{*} n\right)=-1 ; \\
& E((n+i-1) *(n-1)+j, i+j * n)=1 ;
\end{aligned}
$$

end

end

\%Function C 
function $C=\operatorname{get} C(n)$;

$C=\operatorname{zeros}(2 * n *(n-1), 2 * n * n)$

for $\mathrm{i}=1: \mathrm{n}$

for $j=1:(n-1)$

$C\left((i-1) *(n-1)+j,(i-1)^{*} n+j\right)=0.5 ;$

$C((i-1) *(n-1)+j,(i-1) * n+j+1)=0.5 ;$

$C((n+i-1) *(n-1)+j, n *(n+j-1)+i)=0.5 ;$

$C((n+i-1) *(n-1)+j, n *(n+j)+i)=0.5$;

end

end 


\section{APPENDIX B -- ZEBRAFISH FOR IN VIVO MODELLING}

MIFT-A TUMOUR MODEL

Microphthalmia-associated transcription factor (MITF) regulates the differentiation and development of melanocytes and retinal pigment epithelium and is also responsible for pigment cell-specific transcription of the melanogenesis enzyme genes. MITF consists of at least five isoforms, MITF-A, MITF-B, MITF-C, MITF-H, and MITF-M, differing at their N-termini and expression patterns (Shibahara et al., 2001). The zebrafish pigment pattern provides several opportunities to study the development of melanocytes from their undifferentiated precursors. Genetic dissection of the adult pigment pattern reveals multiple populations of melanocytes that develop during the transition from larval to adult form (Rawls and Johnson, 2000). Tumour formation is characterized by clustering of melanocytes. The images below taken in a standard FLIM-TCSPC system show cells transfected with GFP tagged MIFT-A proteins at different stages in the Zebrafish development. 

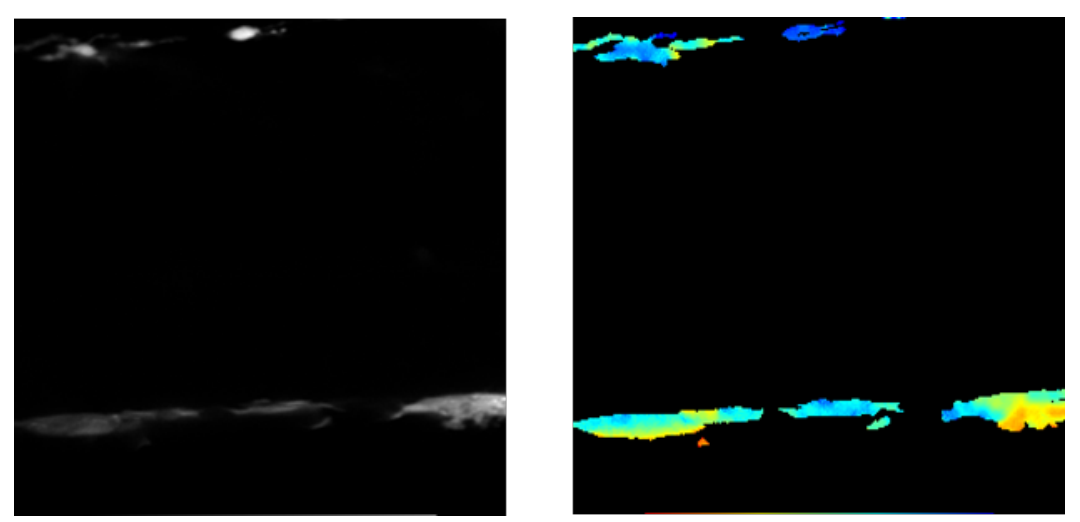

Fluorescence Lifetime Histogram of GFP-Mift-A in a Zebrafish

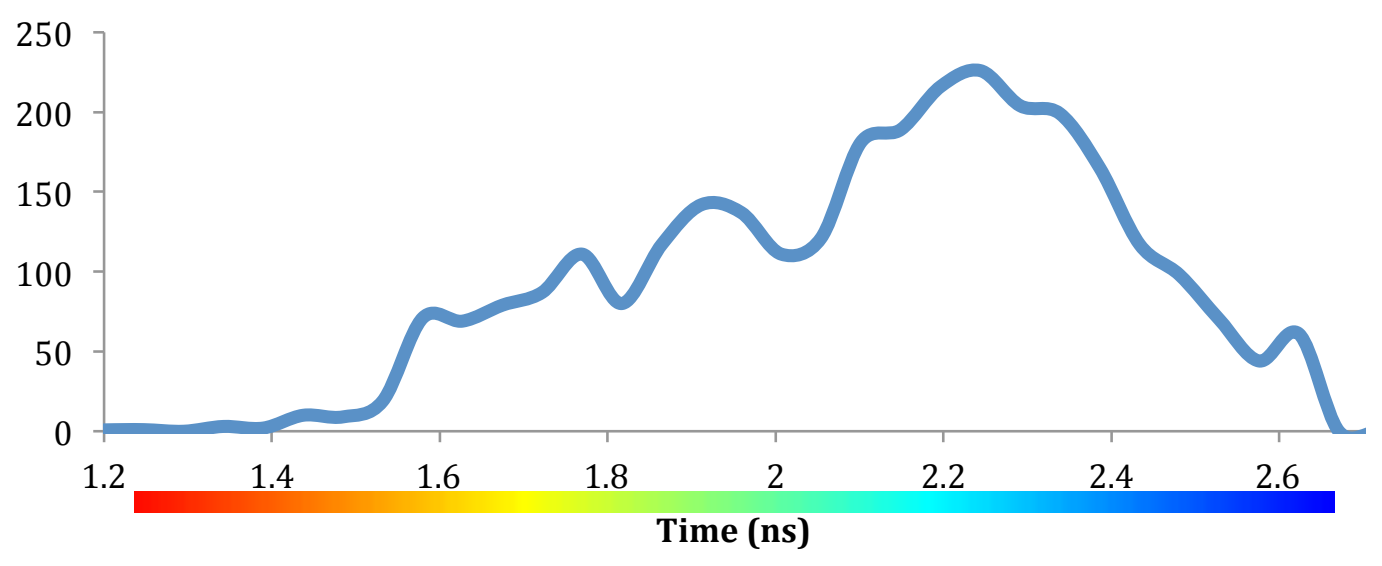

Figure 81: Intensity (left) and FLIM (right) measurements of MIFT-A GFP tagged distribution in a 3 day old Zebrafish accompanied by the Fluorescence Lifetime Histogram below. 

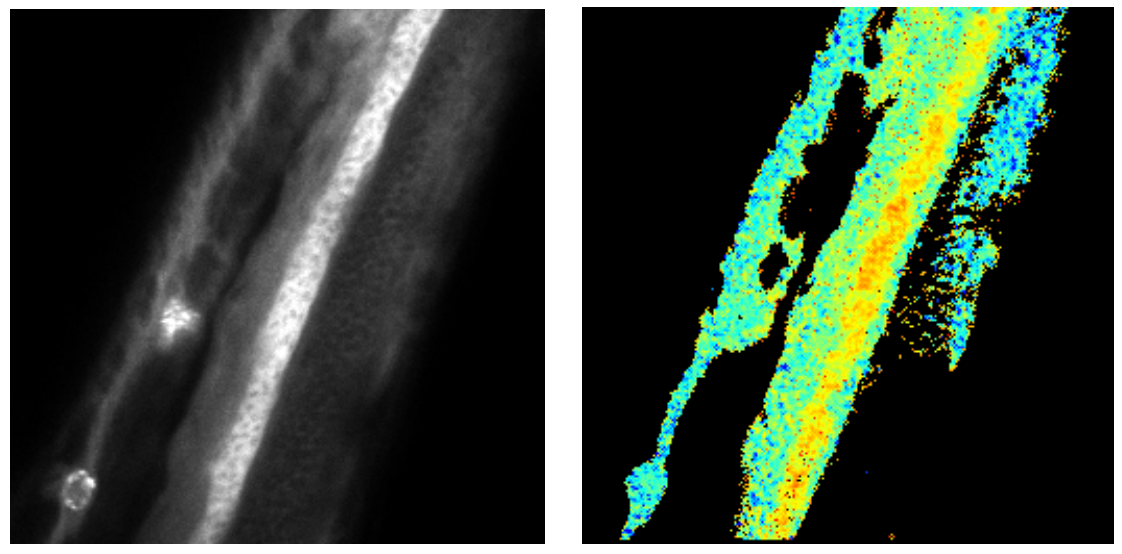

Fluorescence Lifetime Histogram for 5 day old Zebrafish with GFP-MIFT-A

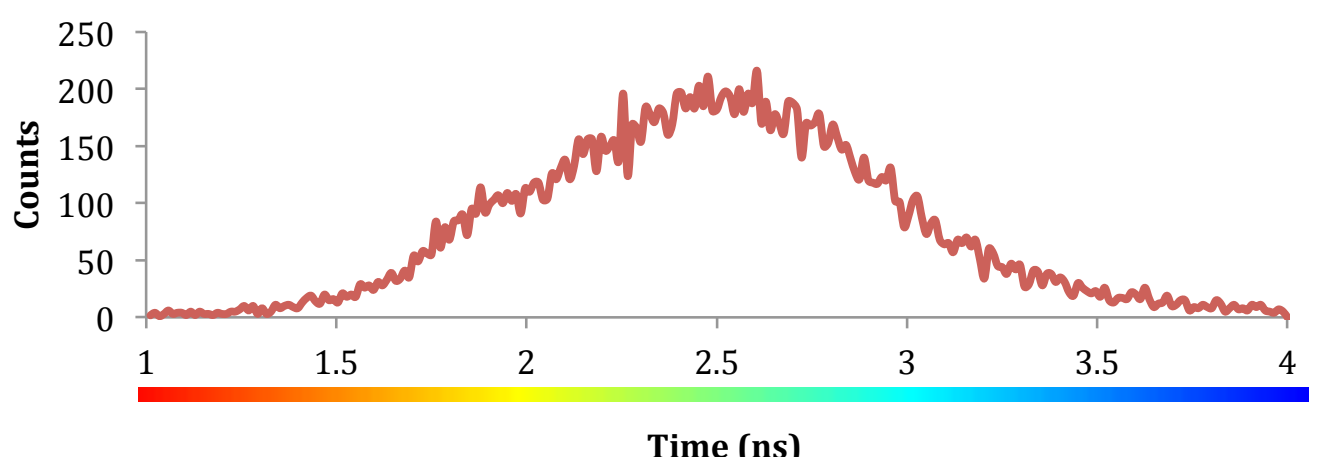

Figure 82: Intensity (left) and FLIM (right) measurements of MIFT-A GFP tagged distribution in a 5 day old Zebrafish accompanied by the Fluorescence Lifetime Histogram below.

The work presented is currently still under investigation. Therefore these preliminary results demonstrate a few avenues of exploration of Zebrafish under various situations. Via the FLIM image MIFT-A GFP tagged cells do not clearly indicate a clustering effect as the GFP is spread throughout the animal. However in the Intensity image, there is an obvious concentration in specific sites as indicated by the high intensity. These sites have been shown to contain solely GFP. Potentially there is clustering at specific sites however further investigation such as a time lapse of the clustering over the Zebrafish's melanocyte growth cycle would further confirm the hypothesis. 


\section{AUTOFLUORESCENCE FILTERING}

During the imaging of the zebrafish a large autofluorescence component was detected and temporally filtered. Significant autofluorescence is common in zebrafish and temporal filtering is trivial filtering due to the characteristic short lifetime (McGinty et al., 2011).
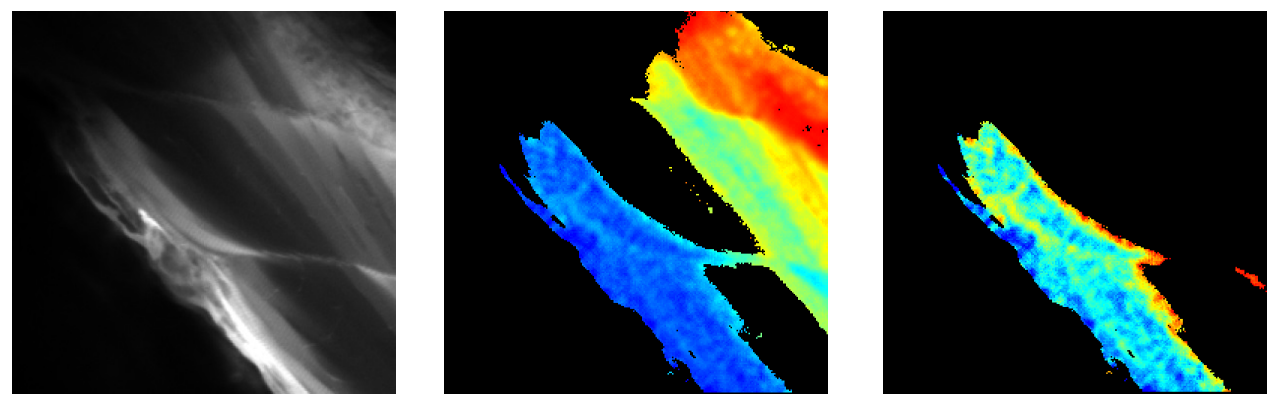

Fluorescence Lifetime Histogram - Full Range

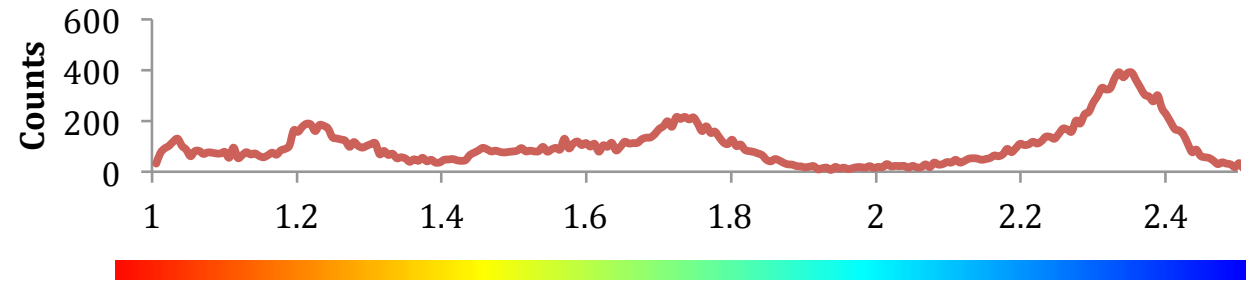

Time (ns)

Fluorescence Lifetime Histogram - Temporaly Filtered

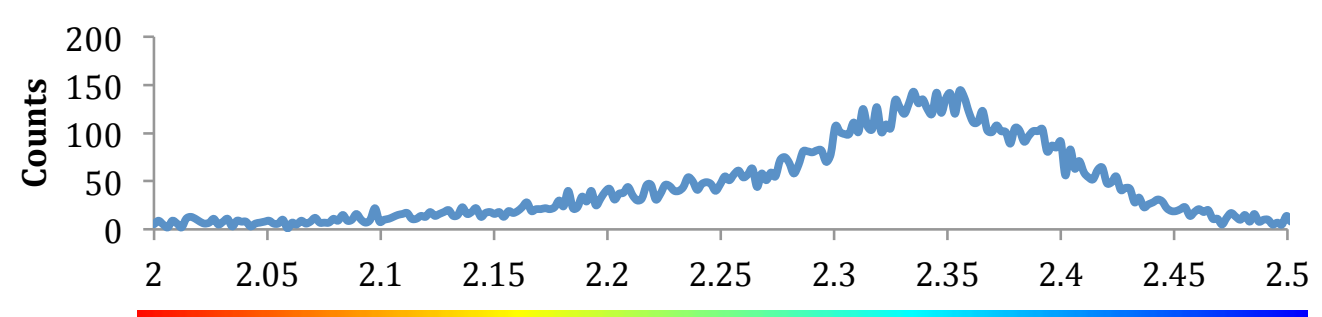

Time (ns)

Figure 83: Temporal filtering of the autofluorescence. Intensity image acquired (left), FLIM image acquired (middle) and autofluorescence removed using temporal filtering (right). Fluorescent lifetime histograms over the full range and the temporally filtered range demonstrated below. 


\section{REFERENCES}

Abbe, E. (1873). Beiträge zur Theorie des Mikroskops und der mikroskopischen Wahrnehmung. Archiv für Mikroskopische Anatomie 9, 413-418.

Agronskaia, A.V., Tertoolen, L., and Gerritsen, H.C. (2003). High frame rate fluorescence lifetime imaging. Journal of Physics D-Applied Physics 36, 16551662.

Ahn, J., Efimov, A., Averitt, R., and Taylor, A. (2003). Terahertz waveform synthesis via optical rectification of shaped ultrafast laser pulses. Optics express 11, 2486-2496.

Ai, H.W., Hazelwood, K.L., Davidson, M.W., and Campbell, R.E. (2008). Fluorescent protein FRET pairs for ratiometric imaging of dual biosensors. Nature methods 5, 401-403.

Ameer-Beg, S.M., Barber, P.R., Hodgkiss, R.J., Locke, R.J., Newman, R.G., Tozer, G.M., Vojnovic, B., and Wilson, J. (2002). Application Of Multiphoton steady state and lifetime imaging to mapping of tumour vascular architecture in vivo. Multiphoton Microscopy in the Biomedical Sciences Ii 4620, 85-95.

Amos, B. (2000). Lessons from the history of light microscopy. Nature Cell Biol 2, E151-152.

Amos, W.B., and White, J.G. (2003). How the confocal laser scanning microscope entered biological research. Biology of the cell / under the auspices of the European Cell Biology Organization 95, 335-342.

Aoki, K., and Matsuda, M. (2009). Visualization of small GTPase activity with fluorescence resonance energy transfer-based biosensors. Nature protocols 4 , 1623-1631.

Arai, Y., and Ikeno, M. (1996). A time digitizer CMOS gate-array with a 250 ps time resolution. Ieee Journal of Solid-State Circuits 31, 212-220.

Armitage, D., Underwood, I., and Wu, S.-T. (2006). Introduction to Microdisplays 1edn (Wiley).

Ashworth, R., and Brennan, C. (2005). Use of transgenic zebrafish reporter lines to study calcium signalling in development. Briefings in functional genomics \& proteomics 4, 186-193.

Aull, B., Loomis, A., Young, D., Heinrichs, R., Felton, B., and Daniels, D. (2002). Geiger-mode avalanche photodiodes for three-dimensional imaging. The Lincoln Laboratory journal 13.

Babcock, H. (1953). The Possibility of Compensating Astronomical Seeing. Publications of the Astronomical Society of the Pacific, 65. 
Bahlmann, K., So, P.T., Kirber, M., Reich, R., Kosicki, B., McGonagle, W., and Bellve, K. (2007). Multifocal multiphoton microscopy (MMM) at a frame rate beyond $600 \mathrm{~Hz}$. Optics express 15, 10991-10998.

Ball, C. (1966). The Early History of the Compound Microscope. Bios 37, 51-60.

Barber, P.R., Ameer-Beg, S.M., Gilbey, J., Carlin, L.M., Keppler, M., Ng, T.C., and Vojnovic, B. (2009). Multiphoton time-domain fluorescence lifetime imaging microscopy: practical application to protein-protein interactions using global analysis. Journal of The Royal Society Interface 6, S93-S105.

Barber, P.R., Ameer-Beg, S.M., Gilbey, J.D., Edens, R.J., Ezike, I., and Vojnovic, B. (2005). Global and pixel kinetic data analysis for FRET detection by multiphoton time-domain FLIM. Proceedings of SPIE, 5700.

Barber, P.R., Pierce, G.P., Ameer-Beg, S.M., Matthews, D.R., Carlin, L.M., Keppler, M., Kelleher, M., Festy, F., Gillett, C., Springall, R., et al. (2008). Towards highthroughput flim for protein-protein interaction screening of live cells and tissue microarrays. 2008 Ieee International Symposium on Biomedical Imaging: From Nano to Macro, Vols 1-4, 356-359.

Bastiaens, P.I., and Pepperkok, R. (2000). Observing proteins in their natural habitat: the living cell. Trends in biochemical sciences 25, 631-637.

Becker, S. (2008). The bh TCSPC handbook, 4 edn (Becker \& Hickl).

Becker, W. (2005). Advanced Time-Correlated Single Photon Counting Techniques, Vol 81 (Springer Science \& Business Media).

Becker, W., Bergmann, A., Hink, M.A., Konig, K., Benndorf, K., and Biskup, C. (2004). Fluorescence lifetime imaging by time-correlated single-photon counting. Microscopy research and technique 63, 58-66.

Becker, W., Su, B., Holub, O., and Weisshart, K. (2011). FLIM and FCS detection in laser-scanning microscopes: increased efficiency by GaAsP hybrid detectors. Microscopy research and technique 74, 804-811.

Berezin, M.Y., and Achilefu, S. (2010). Fluorescence Lifetime Measurements and Biological Imaging. Chemical Reviews 110, 2641-2684.

Bergeron, A., Gauvin, J., Gagnon, F., Gingras, D., Arsenault, H.H., and Doucet, M. (1995). Phase calibration and applications of a liquid-crystal spatial light modulator. Applied Optics 34, 5133-5139.

Betta, G.D., Pancheri, L., Stoppa, D., Henderson, R., and Richardson, J. (2011). Avalanche Photodiodes in Submicron CMOS Technologies for High-Sensitivity Imaging. In Advaches in Photodiodes, G.D. Betta, ed. (InTech).

Bewersdorf, J., Pick, R., and Hell, S.W. (1998). Multifocal multiphoton microscopy. Optics letters 23, 655-657. 
Bhatia, A.B., and Wolf, E. (1954). On the Circle Polynomials of Zernike and Related Orthogonal Sets. Proceedings of the Cambridge Philosophical Society $50,40-48$.

Bianchi, S., and Di Leonardo, R. (2010). Real-time optical micro-manipulation using optimized holograms generated on the GPU. Computer Physics Communications 181, 1442-1446.

Biran, R., Martin, D.C., and Tresco, P.A. (2007). The brain tissue response to implanted silicon microelectrode arrays is increased when the device is tethered to the skull. Journal of biomedical materials research Part A 82, 169178.

Biskup, C., Zimmer, T., and Benndorf, K. (2004). FRET between cardiac Na+ channel subunits measured with a confocal microscope and a streak camera. Nature biotechnology 22, 220-224.

Böhmer, M., Pampaloni, F., Wahl, M., Rahn, H.-J., Erdmann, R., and Enderlein, J. (2001). Time-resolved confocal scanning device for ultrasensitive fluorescence detection. Review of Scientific Instruments 72, 4145-4152.

Booth, M.J. (2007). Adaptive optics in microscopy. Philosophical Transactions of the Royal Society A: Mathematical, Physical and Engineering Sciences 365, 2829-2843.

Booth, M.J., Neil, M.A., Juskaitis, R., and Wilson, T. (2002). Adaptive aberration correction in a confocal microscope. Proceedings of the National Academy of Sciences of the United States of America 99, 5788-5792.

Booth, M.J., Neil, M.A.A., and Wilson, T. (1998a). Aberration correction for confocal imaging in refractive-index-mismatched media. Journal of microscopy 192, 90-98.

Booth, M.J., Neil, M.A.A., and Wilson, T. (1998b). Aberration correction for confocal imaging in refractive-index-mismatched media. Journal of MicroscopyOxford 192, 90-98.

Born, M., and Wolf, E. (1999). Principles of Optics, 7 edn (Cambridge University Press).

Boucrot, E., and Kirchhausen, T. (2008). Mammalian Cells Change Volume during Mitosis. PloS one 3, e1477.

Bourgenot, C., Saunter, C.D., Taylor, J.M., Girkin, J.M., and Love, G.D. (2012). 3D adaptive optics in a light sheet microscope. Optics express 20,13252-13261.

Bowman, R.W., Gibson, G., Carberry, D., Picco, L., Miles, M., and Padgett, M.J. (2011). iTweezers: optical micromanipulation controlled by an Apple iPad. Journal of Optics 13, 044002.

Bowman, R.W., Wright, A.J., and Padgett, M.J. (2010). An SLM-based ShackHartmann wavefront sensor for aberration correction in optical tweezers. Journal of Optics 12, 124004. 
Bradley, D.J., Hutchinson, M.H.R., Koetser, H., Morrow, T., New, G.H.C., and Petty, M.S. (1972). Interactions of Picosecond Laser Pulses with Organic Molecules. I. Two-Photon Fluorescence Quenching and Singlet States Excitation in Rhodamine Dyes, Vol 328.

Bugiel, I., Konig, K., and Wabnitz, H. (1989). Investigation of Cells by Fluorescence Laser Scanning Microscopy with subnanosecond Time Resolution. Lasers in the Life Sciences 3, 47-53.

Buist, Müller, Squier, and Brakenhoff (1998). Real time two-photon absorption microscopy using multi point excitation. Journal of microscopy 192, 217-226.

Buurman, E.P., Sanders, R., Draaijer, A., Gerritsen, H.C., van Veen, J.J.F., Houpt, P.M., and Levine, Y.K. (1992). Fluorescence lifetime imaging using a confocal laser scanning microscope. Scanning 14, 155-159.

Cahalan, M.D., Parker, I., Wei, S.H., and Miller, M.J. (2002). Two-photon tissue imaging: seeing the immune system in a fresh light. Nature reviews Immunology 2, 872-880.

Campagnola, P.J., and Loew, L.M. (2003). Second-harmonic imaging microscopy for visualizing biomolecular arrays in cells, tissues and organisms. Nature biotechnology 21, 1356-1360.

Campagnola, P.J., Wei, M.D., Lewis, A., and Loew, L.M. (1999). High-resolution nonlinear optical imaging of live cells by second harmonic generation. Biophysical journal 77, 3341-3349.

Carvalho, L.A. (2005). Accuracy of Zernike polynomials in characterizing optical aberrations and the corneal surface of the eye. Investigative ophthalmology \& visual science 46, 1915-1926.

Centonze, V.E., and White, J.G. (1998). Multiphoton excitation provides optical sections from deeper within scattering specimens than confocal imaging. Biophysical journal 75, 2015-2024.

Cervino, A., Hosking, S.L., Montes-Mico, R., and Bates, K. (2007). Clinical ocular wavefront analyzers. Journal of refractive surgery (Thorofare, NJ : 1995) 23, 603-616.

Chen, Y., Mills, J.D., and Periasamy, A. (2003). Protein localization in living cells and tissues using FRET and FLIM. Differentiation; research in biological diversity $71,528-541$.

Chen, Y., and Periasamy, A. (2004). Characterization of two-photon excitation fluorescence lifetime imaging microscopy for protein localization. Microscopy research and technique $63,72-80$.

Cheng, A., Gonçalves, J.T., Golshani, P., Arisaka, K., and Portera-Cailliau, C. (2011). Simultaneous 2-photon calcium imaging at different cortical depths in vivo with spatiotemporal multiplexing. Nature methods $8,139-142$. 
Chiangga, S., Zarda, P., Jennewein, T., and Weinfurter, H. (1999). Towards practical quantum cryptography. Applied Physics B 69, 389-393.

Chmyrov, A., Keller, J., Grotjohann, T., Ratz, M., d'Este, E., Jakobs, S., Eggeling, C., and Hell, S.W. (2013). Nanoscopy with more than 100,000 'doughnuts'. Nature methods 10, 737-740.

Choy, E., Chiu, V.K., Silletti, J., Feoktistov, M., Morimoto, T., Michaelson, D., Ivanov, I.E., and Philips, M.R. (1999). Endomembrane trafficking of ras: the CAAX motif targets proteins to the ER and Golgi. Cell 98, 69-80.

Clegg, R.M. (1992). Fluorescence resonance energy transfer and nucleic acids. Methods Enzymol 211, 353-388.

Clegg, R.M. (1995). Fluorescence resonance energy transfer. Current opinion in biotechnology 6, 103-110.

Coelho, S., Poland, S., Krstajic, N., Li, D., Monypenny, J., Walker, R., Tyndall, D., Ng, T., Henderson, R., and Ameer-Beg, S. (2013). Multifocal multiphoton microscopy with adaptive optical correction. Proceedings of SPIE, 8588

Cohn, R.W. (2001). Fundamental properties of spatial light modulators for the approximate optical computation of Fourier transforms: a review. Optical Engineering 40, 2452-2463.

Colyer, R.A., Lee, C., and Gratton, E. (2008). A novel fluorescence lifetime imaging system that optimizes photon efficiency. Microscopy research and technique $71,201-213$.

Colyer, R.A., Scalia, G., Villa, F.A., Guerrieri, F., Tisa, S., Zappa, F., Cova, S., Weiss, S., and Michalet, X. (2011). Ultra high-throughput single molecule spectroscopy with a 1024 pixel SPAD. Proceedings of SPIE 7905,.

Costa, D.M., and Ferrer-Blasco, T. (2010). Adaptive optics, wavefront aberrations and visual simulation.

Cova, S., Ghioni, M., Lacaita, A., Samori, C., and Zappa, F. (1996). Avalanche photodiodes and quenching circuits for single-photon detection. Applied Optics 35, 1956-1976.

Cova, S., Lacaita, A., Ghioni, M., and Ripamonti, G. (1989). High-accuracy picosecond characterization of gain-switched laser diodes. Optics letters 14, 1341-1343.

Dai, G. (2008). Wavefront Optics for Vision Correction (SPIE).

Dai, G. (2006). Zernike aberration coefficients transformed to and from Fourier series coefficients for wavefront representation. Optics letters 31, 501-503.

Dainty, C. (2007). Adaptive Optics fo Industry and Medicine (Imperial College Press). 
De Heyn, V., Van der Plas, G., Ryckaert, J., and Craninckx, J. (2007). A fast startup $3 \mathrm{GHz}-10 \mathrm{GHz}$ digitally controlled oscillator for UWB impulse radio in $90 \mathrm{~nm}$ CMOS. IEEE, 13, 484-487

Débarre, D., Botcherby, E.J., Booth, M.J., and Wilson, T. (2008). Adaptive optics for structured illumination microscopy. Optics express 16, 9290-9305.

Débarre, D., Botcherby, E.J., Watanabe, T., Srinivas, S., Booth, M.J., and Wilson, T. (2009). Image-based adaptive optics for two-photon microscopy. Optics letters 34, 2495-2497.

Denk, W., Strickler, J.H., and Webb, W.W. (1990). Two-photon laser scanning fluorescence microscopy. Science 248, 73-76.

Denk, W., and Svoboda, K. (1997). Photon upmanship: Why multiphoton imaging is more than a gimmick. Neuron 18, 351-357.

Di Leonardo, R., Ianni, F., and Ruocco, G. (2007). Computer generation of optimal holograms for optical trap arrays. Optics express 15, 1913-1922.

Diaspro, A., Bianchini, P., Vicidomini, G., Faretta, M., Ramoino, P., and Usai, C. (2006). Multi-photon excitation microscopy. Biomedical engineering online 5, 36.

Diaspro, A., Chirico, G., and Collini, M. (2005). Two-photon fluorescence excitation and related techniques in biological microscopy. Quarterly reviews of biophysics 38, 97-166.

Dong, C.Y., Koenig, K., and So, P. (2003). Characterizing point spread functions of two-photon fluorescence microscopy in turbid medium. Journal of biomedical optics 8, 450-459.

Dowling, K., Dayel, M.J., Lever, M.J., French, P.M.W., Hares, J.D., and DymokeBradshaw, A.K.L. (1998). Fluorescence lifetime imaging with picosecond resolution for biomedical applications. Optics letters $23,810-812$.

Drobizhev, M., Makarov, N.S., Tillo, S.E., Hughes, T.E., and Rebane, A. (2011). Two-photon absorption properties of fluorescent proteins. Nature methods 8 , 393-399.

Duncan, R.R., Bergmann, A., Cousin, M.A., Apps, D.K., and Shipston, M.J. (2004). Multi-dimensional time-correlated single photon counting (TCSPC) fluorescence lifetime imaging microscopy (FLIM) to detect FRET in cells. Journal of microscopy $215,1-12$.

Dyba, M., Jakobs, S., and Hell, S.W. (2003). Immunofluorescence stimulated emission depletion microscopy. Nature Biotech 21, 1303-1304.

Espinosa, J., Mas, D., Perez, J., and Illueca, C. (2010). Optical surface reconstruction technique through combination of zonal and modal fitting. Journal of biomedical optics 15, 026022. 
Esposito, A., Bader, A.N., Schlachter, S.C., van den Heuvel, D.J., Schierle, G.S., Venkitaraman, A.R., Kaminski, C.F., and Gerritsen, H.C. (2011). Design and application of a confocal microscope for spectrally resolved anisotropy imaging. Optics express 19, 2546-2555.

Esposito, A., Federici, F., Usai, C., Cannone, F., Chirico, G., Collini, M., and Diaspro, A. (2004). Notes on theory and experimental conditions behind two-photon excitation microscopy. Microscopy research and technique 63, 12-17.

Fan, G.Y., Fujisaki, H., Miyawaki, A., Tsay, R.K., Tsien, R.Y., and Ellisman, M.H. (1999). Video-Rate Scanning Two-Photon Excitation Fluorescence Microscopy and Ratio Imaging with Cameleons. Biophysical journal 76, 2412-2420.

Festy, F., Ameer-Beg, S.M., Ng, T., and Suhling, K. (2007). Imaging proteins in vivo using fluorescence lifetime microscopy. Molecular bioSystems 3, 381-391.

Fritz, R.D., Letzelter, M., Reimann, A., Martin, K., Fusco, L., Ritsma, L., Ponsioen, B., Fluri, E., Schulte-Merker, S., van Rheenen, J., et al. (2013). A versatile toolkit to produce sensitive FRET biosensors to visualize signaling in time and space. Science signaling 6, rs12.

Fruhwirth, G.O., Fernandes, L.P., Weitsman, G., Patel, G., Kelleher, M., Lawler, K., Brock, A., Poland, S.P., Matthews, D.R., Keri, G., et al. (2011). How Forster resonance energy transfer imaging improves the understanding of protein interaction networks in cancer biology. Chemphyschem : a European journal of chemical physics and physical chemistry $12,442-461$.

Fukasawa, A., Haba, J., Kageyama, A., Nakazawa, H., and Suyama, M. (2008). High Speed HPD for Photon Counting. IEEE Transactions on Nuclear Science 55, 758762.

Gadella Jr, T.W.J., Jovin, T.M., and Clegg, R.M. (1993). Fluorescence lifetime imaging microscopy (FLIM): Spatial resolution of microstructures on the nanosecond time scale. Biophysical Chemistry 48, 221-239.

Ganesan, S., Ameer-beg, S.M., Ng, T.T.C., Vojnovic, B., and Wouters, F.S. (2006). A dark yellow fluorescent protein (YFP)-based Resonance Energy-Accepting Chromoprotein (REACh) for Förster resonance energy transfer with GFP. Proceedings of the National Academy of Sciences of the United States of America 103, 4089-4094.

Gao, J., Liao, J., and Yang, G.-Y. (2009). CAAX-box protein, prenylation process and carcinogenesis. American Journal of Translational Research 1, 312-325.

Gauderon, R., Lukins, P.B., and Sheppard, C.J. (1999). Effect of a confocal pinhole in two-photon microscopy. Microscopy research and technique 47, 210-214.

Gerchberg, R.W. (1972). Holography without Fringes in the Electron Microscope. Nature 240, 404-406.

Gerchberg, R.W., and Saxton, W. (1972). A practical algorithm for the determination of the phase from image and diffraction plane pictures. Optik 35 . 
Gerritsen, H.C., Asselbergs, M.A., Agronskaia, A.V., and Van Sark, W.G. (2002). Fluorescence lifetime imaging in scanning microscopes: acquisition speed, photon economy and lifetime resolution. Journal of microscopy 206, 218-224.

Gersbach, M., Maruyama, Y., Labonne, E., Richardson, J., Walker, R., Grant, L., Henderson, R., Borghetti, F., Stoppa, D., and Charbon, E. (2009). A parallel 32x32 time-to-digital converter array fabricated in a $130 \mathrm{~nm}$ imaging CMOS technology. Proceedings of ESSCIRC.

Gersbach, M., Trimananda, R., Maruyama, Y., Fishburn, M., Stoppa, D., Richardson, J., Walker, J.M., Henderson, R., and Charbon, E. (2010). High Framerate TCSPC-FLIM Using a Novel SPAD-based Image Sensor. Proceedings of SPIE, 7780

Gil, A., Segura, J., and Temme, N.M. (2007). Numerical Methods for Special Functions (Society for Industrial and Applied Mathematics).

Girkin, J.M., Poland, S., and Wright, A.J. (2009). Adaptive optics for deeper imaging of biological samples. Current opinion in biotechnology 20, 106-110.

Godara, P., Dubis, A.M., Roorda, A., Duncan, J.L., and Carroll, J. (2010). Adaptive optics retinal imaging: emerging clinical applications. Optometry and vision science : official publication of the American Academy of Optometry 87, 930941.

Goodman, J. (1996). Introduction to Fourier Optics (McGraw-Hill).

Göppert-Mayer, M. (1931). Über Elementarakte mit zwei Quantensprüngen. Annalen der Physik 401, 273-294.

Gould, T.J., Burke, D., Bewersdorf, J., and Booth, M.J. (2012). Adaptive optics enables 3D STED microscopy in aberrating specimens. Optics express 20, 20998-21009.

Gould, T.J., Kromann, E.B., Burke, D., Booth, M.J., and Bewersdorf, J. (2013). Autoaligning stimulated emission depletion microscope using adaptive optics. Optics letters $38,1860-1862$.

Grant, D.M., McGinty, J., McGhee, E.J., Bunney, T.D., Owen, D.M., Talbot, C.B., Zhang, W., Kumar, S., Munro, I., Lanigan, P.M., et al. (2007). High speed optically sectioned fluorescence lifetime imaging permits study of live cell signaling events. Optics express 15, 15656-15673.

Grier, D.G. (2003). A revolution in optical manipulation. Nature 424, 810-816.

Guerrieri, F., Tisa, S., Tosi, A., and Zappa, F. (2010). Two-Dimensional SPAD Imaging Camera for Photon Counting. Photonics Journal, IEEE 2, 759-774.

Gulinatti, A., Rech, I., Assanelli, M., Ghioni, M., and Cova, S. (2011). A physically based model for evaluating the photon detection efficiency and the temporal response of SPAD detectors. Journal of Modern Optics 58, 210-224.

Hakamata, T. (2006). Photomultiplier Tubes, 3 edn (Hamamatsu Photonics). 
Hancock, J.F., Paterson, H., and Marshall, C.J. (1990). A polybasic domain or palmitoylation is required in addition to the CAAX motif to localize p21ras to the plasma membrane. Cell 63, 133-139.

Harmening, W.M., Vobig, M.A., Walter, P., and Wagner, H. (2007). Ocular aberrations in barn owl eyes. Vision research 47, 2934-2942.

Hartmann, J. (1900). Bemerkungen über den Bau und die Justirung von Spektrographen. Springer 20, 17-27.

Helmchen, F., and Denk, W. (2005). Deep tissue two-photon microscopy. Nature methods 2, 932-940.

Hendriks, A., Naidoo, D., Roux, F.S., López-Mariscal, C., and Forbes, A. (2012). The generation of flat-top beams by complex amplitude modulation with a phase-only spatial light modulator. Proceedings of SPIE, 8490

Hermerschmidt, A., Osten, S., Krüger, S., and Blümel, T. (2007). Wave front generation using a phase-only modulating liquid-crystal-based micro-display with HDTV resolution. Proceedings of SPIE, 6584

Herrmann, J. (1977). Properties of phase conjugate adaptive optical systems. J Opt Soc Am 67, 290-295.

Howard, S.S., Straub, A., Horton, N.G., Kobat, D., and Xu, C. (2013). Frequencymultiplexed in vivo multiphoton phosphorescence lifetime microscopy. Nature Photon 7, 33-37.

Howland, H.C., and Howland, B. (1977). A subjective method for the measurement of monochromatic aberrations of the eye. J Opt Soc Am 67, 15081518.

Hu, L., Xuan, L., Li, D., Cao, Z., Mu, Q., Liu, Y., Peng, Z., and Lu, X. (2009). Wavefront correction based on a reflective liquid crystal wavefront sensor. Journal of Optics A: Pure and Applied Optics 11, 015511.

Huang, S.-H., Hsiao, C.-D., Lin, D.-S., Chow, C.-Y., Chang, C.-J., and Liau, I. (2011). Imaging of Zebrafish in vivo with Second-Harmonic Generation Reveals Shortened Sarcomeres Associated with Myopathy Induced by Statin. PloS one 6, e24764.

Huntington, A., Compton, M.A., and Williams, G. (2007). Linear-mode singlephoton APD detectors. Proceedings of SPIE 6771

Ingargiola, A., Panzeri, F., Sarkosh, N., Gulinatti, A., Rech, I., Ghioni, M., Weiss, S., and Michalet, X. (2013). 8-spot smFRET analysis using two 8-pixel SPAD arrays. Proceedings of SPIE 8590.

Ishido, N., Kobayashi, Y., Sako, H., Arai, T., Fukuda, M., and Nakamura (2011). How to make FRET biosensors for Rab family GTPases. (InTech) 
Jakobs, S., Subramaniam, V., Schonle, A., Jovin, T.M., and Hell, S.W. (2000). EFGP and DsRed expressing cultures of Escherichia coli imaged by confocal, twophoton and fluorescence lifetime microscopy. FEBS letters 479, 131-135.

Jares-Erijman, E.A., and Jovin, T.M. (2003). FRET imaging. Nature biotechnology 21, 1387-1395.

Ji, N., Milkie, D.E., and Betzig, E. (2010). Adaptive optics via pupil segmentation for high-resolution imaging in biological tissues. Nature methods 7, 141-147.

Ji, N., Sato, T.R., and Betzig, E. (2012). Characterization and adaptive optical correction of aberrations during in vivo imaging in the mouse cortex. Proceedings of the National Academy of Sciences of the United States of America 109, 22-27.

Kaemmerer, M., Mrochen, M., Mierdel, P., Krinke, H.-E., and Seiler, T. (2000). Clinical Experience With the Tscherning Aberrometer. Journal of Refractive Surgery 16, S585.

Kardash, E., Bandemer, J., and Raz, E. (2011). Imaging protein activity in live embryos using fluorescence resonance energy transfer biosensors. Nature protocols 6, 1835-1846.

Kenworthy, A.K. (2001). Imaging protein-protein interactions using fluorescence resonance energy transfer microscopy. Methods 24, 289-296.

Kim, K.H., Buehler, C., Bahlmann, K., Ragan, T., Lee, W.-C.A., Nedivi, E., Heffer, E.L., Fantini, S., and So, P.T.C. (2007). Multifocal multiphoton microscopy based on multianode photomultiplier tubes. Optics express 15, 11658-11678.

Kirkpatrick, N.D., Chung, E., Cook, D.C., Han, X., Gruionu, G., Liao, S., Munn, L.L., Padera, T.P., Fukumura, D., and Jain, R.K. (2012). Video-rate resonant scanning multiphoton microscopy: An emerging technique for intravital imaging of the tumor microenvironment. Intravital 1.

Kner, P., Chhun, B.B., Griffis, E.R., Winoto, L., and Gustafsson, M.G.L. (2009). Super-resolution video microscopy of live cells by structured illumination. Nature methdos 6, 339-342.

Konig, K. (2000). Multiphoton microscopy in life sciences. Journal of microscopy 200, 83-104.

Konig, K., and Halbhuber, K.J. (1998). Introduction of the Guest-Editors with a short review on history of optics in Jena. Cellular and molecular biology (Noisyle-Grand, France) 44, 659-671.

Konig, K., So, P.T.C., Mantulin, W.W., and Gratton, E. (1997). Cellular response to near-infrared femtosecond laser pulses in two-photon microscopes. Optics letters 22, 135-136.

Krishnan, R.V., Masuda, A., Centonze, V.E., and Herman, B. (2003a). Quantitative imaging of protein-protein interactions by multiphoton fluorescence lifetime 
imaging microscopy using a streak camera. Journal of biomedical optics 8,362 367.

Krishnan, R.V., Saitoh, H., Terada, H., Centonze, V.E., and Herman, B. (2003b). Development of a multiphoton fluorescence lifetime imaging microscopy system using a streak camera. Review of Scientific Instruments 74, 2714-2721.

Krstajić, N., Poland, S., Tyndall, D., Walker, R., Coelho, S., Li, D.D., Richardson, J., Ameer-Beg, S., and Henderson, R. (2013). Improving TCSPC data acquisition from CMOS SPAD arrays. Proceedings of SPIE, 8797

Kumar, S., Alibhai, D., Margineanu, A., Laine, R., Kennedy, G., McGinty, J., Warren, S., Kelly, D., Alexandrov, Y., Munro, I., et al. (2011). FLIM FRET technology for drug discovery: automated multiwell-plate high-content analysis, multiplexed readouts and application in situ. Chemphyschem : a European journal of chemical physics and physical chemistry 12, 609-626.

Kumar, S., Dunsby, C., De Beule, P.A., Owen, D.M., Anand, U., Lanigan, P.M., Benninger, R.K., Davis, D.M., Neil, M.A., Anand, P., et al. (2007a). Multifocal multiphoton excitation and time correlated single photon counting detection for 3-D fluorescence lifetime imaging. Optics express 15, 12548-12561.

Kumar, S., Dunsby, C., De Beule, P.A., Owen, D.M., Anand, U., Lanigan, P.M., Benninger, R.K., Davis, D.M., Neil, M.A., Anand, P., et al. (2007b). Multifocal multiphoton excitation and time correlated single photon counting detection for 3-D fluorescence lifetime imaging. Optics express 15, 12548-12561.

Lakowicz, J.R. (1990). Topics in Fluorescence Spectroscopy (Springer).

Lakowicz, J.R. (2006). Principles of Fluorescence Spectroscopy, 3 edn (Springer).

Lakowicz, J.R., Szmacinski, H., Nowaczyk, K., Berndt, K.W., and Johnson, M. (1992a). Fluorescence lifetime imaging. Analytical biochemistry 202, 316-330.

Lakowicz, J.R., Szmacinski, H., Nowaczyk, K., and Johnson, M.L. (1992b). Fluorescence lifetime imaging of free and protein-bound NADH. Proceedings of the National Academy of Sciences of the United States of America 89, 12711275.

Lam, A.J., St-Pierre, F., Gong, Y., Marshall, J.D., Cranfill, P.J., Baird, M.A., McKeown, M.R., Wiedenmann, J., Davidson, M.W., Schnitzer, M.J., et al. (2012). Improving FRET dynamic range with bright green and red fluorescent proteins. Nature methods 9, 1005-1012.

Leach, J., Wulff, K., Sinclair, G., Jordan, P., Courtial, J., Thomson, L., Gibson, G., Karunwi, K., Cooper, J., and Laczik, Z.J. (2006). Interactive approach to optical tweezers control. Applied optics 45, 897-903.

Lenz, M., Brown, A., Auksorius, E., Davis, D., Dunsby, C., Neil, M., and French, P. (2011). A STED-FLIM microscope applied to imaging the natural killer cell immune synapse. Proceedings of SPIE, 7903 
Levitt, J.A., Matthews, D.R., Ameer-Beg, S.M., and Suhling, K. (2009). Fluorescence lifetime and polarization-resolved imaging in cell biology. Current opinion in biotechnology 20, 28-36.

Li, D., Arlt, J., Richardson, J., Walker, R., Buts, A., Stoppa, D., Charbon, E., and Henderson, R. (2010). Real-time fluorescence lifetime imaging system with a $32 \times 320.13 \mu \mathrm{m}$ CMOS low dark-count single-photon avalanche diode array. Optics express 18, 10257-10269.

Li, D., Poland, S., Coelho, S., Tyndall, D., Zhang, W., Richardson, J., Henderson, R., and Ameer-Beg, S. (2012a). Advanced fluorescence lifetime imaging algorithms for CMOS single-photon sensor based multi-focal multi-photon microscopy. Proceedings of IEEE, 3036-3039.

Li, D., Walker, R., Richardson, J., Rae, B., Buts, A., Renshaw, D., and Henderson, R. (2009). Hardware implementation and calibration of background noise for an integration-based fluorescence lifetime sensing algorithm. JOSA A 26, 804-814.

Li, D., Ameer-Beg, S., Arlt, J., Tyndall, D., Walker, R., Matthews, D.R., Visitkul, V., Richardson, J., and Henderson, R.K. (2012b). Time-domain fluorescence lifetime imaging techniques suitable for solid-state imaging sensor arrays. Sensors 12, 5650-5669.

Li, D., Arlt, J., Tyndall, D., Walker, R., Richardson, J., Stoppa, D., Charbon, E., and Henderson, R.K. (2011). Video-rate fluorescence lifetime imaging camera with CMOS single-photon avalanche diode arrays and high-speed imaging algorithm. Journal of biomedical optics 16, 096012.

Liang, J., and Williams, D.R. (1997). Aberrations and retinal image quality of the normal human eye. JOSA A 14, 2873-2883.

Liu, B., Wu, L., and Zhang, J. (2006). Phase-only SLM Generating Variable Patterns Applied in Optical Connection. Journal of Physics: Conference Series, 48, 902.

Luberek, J. (2010). Fourier plane analysis and refinement of SLM calibration (Patent).

Luchowski, R., Kapusta, P., Szabelski, M., Sarkar, P., Borejdo, J., Gryczynski, Z., and Gryczynski, I. (2009). Förster resonance energy transfer (FRET)-based picosecond lifetime reference for instrument response evaluation. Measurement Science and Technology 20, 095601.

Mahajan, V.N. (1994). Zernike circle polynomials and optical aberrations of systems with circular pupils. Applied optics 33, 8121-8121.

Mahou, P., Zimmerley, M., Loulier, K., Matho, K.S., Labroille, G., Morin, X., Supatto, W., Livet, J., Debarre, D., and Beaurepaire, E. (2012). Multicolor twophoton tissue imaging by wavelength mixing. Nature methods 9, 815-818.

Maiti, S., Haupts, U., and Webb, W.W. (1997). Fluorescence correlation spectroscopy: diagnostics for sparse molecules. Proceedings of the National Academy of Sciences 94, 11753-11757. 
Marangoni, S., Rech, I., Ghioni, M., Maccagnani, P., Chiari, M., Cretich, M., Damin, F., Di Carlo, G., and Cova, S. (2010). A 6 x 8 photon-counting array detector system for fast and sensitive analysis of protein microarrays. Sensors and Actuators B-Chemical 149, 420-426.

Markovic, B., Tisa, S., Villa, F.A., Tosi, A., and Zappa, F. (2013). A high-linearity, 17 ps precision time-to-digital converter based on a single-stage Vernier delay loop fine interpolation. IEEE Transactions on Circuits and Systems I: Regular Papers 60, 557-569.

Marsh, P., Burns, D., and Girkin, J. (2003). Practical implementation of adaptive optics in multiphoton microscopy. Optics express 11, 1123-1130.

Matthews, D.R., Fruhwirth, G.O., Weitsman, G., Carlin, L.M., Ofo, E., Keppler, M., Barber, P.R., Tullis, I.D., Vojnovic, B., and Ng, T. (2012). A multi-functional imaging approach to high-content protein interaction screening. PloS one 7, e33231.

Matthews, J.L. (2009). Common diseases of laboratory zebrafish. In Essential Zebrafish Methods: Genetics and Genomics: Genetics and Genomics, H. Detrich, ed. (Elsevier), p. 321.

Mayor, S., and Bilgrami, S. (2007). Fretting about FRET in cell and structural biology. Evaluating Techniques in Biochemical Re search, Cell Press, Cambridge.

McGinty, J., Stuckey, D.W., Soloviev, V.Y., Laine, R., Wylezinska-Arridge, M., Wells, D.J., Arridge, S.R., French, P.M., Hajnal, J.V., and Sardini, A. (2011). In vivo fluorescence lifetime tomography of a FRET probe expressed in mouse. Biomedical optics express 2, 1907-1917.

McGinty, J., Tahir, K.B., Laine, R., Talbot, C.B., Dunsby, C., Neil, M.A., Quintana, L., Swoger, J., Sharpe, J., and French, P.M. (2008). Fluorescence lifetime optical projection tomography. Journal of biophotonics 1, 390-394.

Michalet, X., Cheng, A., Antelman, J., Suyama, M., Arisaka, K., and Weiss, S. (2008). Hybrid photodetector for single-molecule spectroscopy and microscopy. Proc Soc Opt Instrum Eng, 68620-68629.

Michalet, X., Colyer, R., Scalia, G., Ingargiola, A., Lin, R., Millaud, J., Weiss, S., Siegmund, O.H., Tremsin, A.S., and Vallerga, J.V. (2013). Development of new photon-counting detectors for single-molecule fluorescence microscopy. Philosophical Transactions of the Royal Society B: Biological Sciences 368, 20120035.

Milkie, D.E., Betzig, E., and Ji, N. (2011). Pupil-segmentation-based adaptive optical microscopy with full-pupil illumination. Optics letters 36, 4206-4208.

Mochizuki, N., Yamashita, S., Kurokawa, K., Ohba, Y., Nagai, T., Miyawaki, A., and Matsuda, M. (2001). Spatio-temporal images of growth-factor-induced activation of Ras and Rap1. Nature 411, 1065-1068.

Moreno, I., Lizana, A., Márquez, A., Iemmi, C., Fernández, E., Campos, J., and Yzuel, M. (2008). Time fluctuations of the phase modulation in a liquid crystal 
on silicon display: characterization and effects in diffractive optics. Optics express 16, 16711-16722.

Mrochen, M., Kaemmerer, M., Mierdel, P., Krinke, H.-E., and Seiler, T. (2000). Principles of Tscherning aberrometry. Journal of Refractive Surgery 16, S570S571.

Mukhtar, E., Bergström, F., and Johansson, L.B.-Å. (2002). Hyper Rayleigh scattering yields improved response function in analysing 2-photon excited fluorescence. Journal of Fluorescence 12, 481-484.

Neal, D.R., Copland, J., and Neal, D.A. (2002). Shack-Hartmann wavefront sensor precision and accuracy. Proceedings of International Symposium on Optical Science and Technology, 148-160.

Neil, M., Booth, M., and Wilson, T. (2000a). Closed-loop aberration correction by use of a modal Zernike wave-front sensor. Optics letters 25, 1083-1085.

Neil, M., Juskaitis, R., Booth, M., Wilson, T., Tanaka, T., and Kawata, S. (2000b). Adaptive aberration correction in a two - photon microscope. Journal of microscopy 200, 105-108.

Ng, T., Squire, A., Hansra, G., Bornancin, F., Prevostel, C., Hanby, A., Harris, W., Barnes, D., Schmidt, S., and Mellor, H. (1999). Imaging protein kinase $C \alpha$ activation in cells. Science 283, 2085-2089.

Niclass, C., Favi, C., Kluter, T., Gersbach, M., and Charbon, E. (2008). A 128128 single-photon image sensor with column-level 10-bit time-to-digital converter array. Solid-State Circuits, IEEE Journal of 43, 2977-2989.

Niclass, C., Gersbach, M., Henderson, R., Grant, L., and Charbon, E. (2007). A single photon avalanche diode implemented in 130-nm CMOS technology. Selected Topics in Quantum Electronics, IEEE Journal of 13, 863-869.

Niclass, C., Rochas, A., Besse, P.-A., and Charbon, E. (2005). Design and characterization of a CMOS 3-D image sensor based on single photon avalanche diodes. Solid-State Circuits, IEEE Journal of 40, 1847-1854.

Nielsen, T., Fricke, M., Hellweg, D., and Andresen, P. (2001). High efficiency beam splitter for multifocal multiphoton microscopy. Journal of microscopy 201, 368-376.

Nightingale, N. (1990). A new silicon avalanche photodiode photon counting detector module for astronomy. Experimental Astronomy 1, 407-422.

Nikolenko, V., Watson, B.O., Araya, R., Woodruff, A., Peterka, D.S., and Yuste, R. (2008). SLM microscopy: scanless two-photon imaging and photostimulation with spatial light modulators. Frontiers in neural circuits 2 .

Noll, R.J. (1976). Zernike polynomials and atmospheric turbulence. JOsA 66, 207-211. 
Osten, S., Krüger, S., and Hermerschmidt, A. (2007). New HDTV (1920x1080) phase-only SLM. Proceedings of SPIE, 6487.

Padilla-Parra, S., Audugé, N., Lalucque, H., Mevel, J.-C., Coppey-Moisan, M., and Tramier, M. (2009). Quantitative comparison of different fluorescent protein couples for fast FRET-FLIM acquisition. Biophysical journal 97, 2368-2376.

Panagopoulou, S.I., and Neal, D.R. (2004). Zonal matrix iterative method for wavefront reconstruction from gradient measurements. Journal of refractive surgery (Thorofare, NJ: 1995) 21, S563-569.

Panagopoulou, S.I., and Neal, D.R. (2014). Zernike vs. Zonal Matrix Iterative Wavefront Reconstructor.

Panzeri, F., Ingargiola, A., Lin, R.R., Sarkhosh, N., Gulinatti, A., Rech, I., Ghioni, M., Cova, S., Weiss, S., and Michalet, X. (2013). Single-molecule FRET experiments with a red-enhanced custom technology SPAD. Proceedings of SPIE, 8590.

Parsons, M., Monypenny, J., Ameer-Beg, S.M., Millard, T.H., Machesky, L.M., Peter, M., Keppler, M.D., Schiavo, G., Watson, R., and Chernoff, J. (2005). Spatially distinct binding of Cdc42 to PAK1 and N-WASP in breast carcinoma cells. Molecular and cellular biology 25, 1680-1695.

Patterson, G.H., and Piston, D.W. (2000). Photobleaching in two-photon excitation microscopy. Biophysical journal 78, 2159-2162.

Pawley, J. (2006). Handbook of Biological Confocal Microscopy, 3 edn (Springer).

Pepper, D.M. (1986). Applications of optical phase conjugation. Scientific American 254, 74-83.

Peter, M., Ameer-Beg, S.M., Hughes, M.K., Keppler, M.D., Prag, S., Marsh, M., Vojnovic, B., and Ng, T. (2005). Multiphoton-FLIM quantification of the EGFPmRFP1 FRET pair for localization of membrane receptor-kinase interactions. Biophysical journal 88, 1224-1237.

Peter, M., and Ameer - Beg, S.M. (2004). Imaging molecular interactions by multiphoton FLIM. Biology of the Cell 96, 231-236.

Peticolas, W.L., Goldsborough, J.P., and Rieckhoff, K. (1963). Double photon excitation in organic crystals. Physical Review Letters 10, 43.

Piston, D.W., and Kremers, G.-J. (2007). Fluorescent protein FRET: the good, the bad and the ugly. Trends in biochemical sciences 32, 407-414.

Platt, B.C. (2001). History and principles of Shack-Hartmann wavefront sensing. Journal of Refractive Surgery 17, S573-S577.

Poland, S.P., Coelho, S., Krstajić, N., Tyndall, D., Walker, R., Monypenny, J., Li, D.D., Henderson, R., and Ameer-Beg, S. (2013). Development of a fast TCSPC FLIMFRET imaging system. Proceedings of SPIE, 8588. 
Poland, S.P., Krstajic, N., Coelho, S., Tyndall, D., Walker, R.J., Devauges, V., Morton, P.E., Nicholas, N.S., Richardson, J., Li, D.D., et al. (2014). Time-resolved multifocal multiphoton microscope for high speed FRET imaging in vivo. Optics letters 39, 6013-6016.

Pollok, B.A., and Heim, R. (1999). Using GFP in FRET-based applications. Trends in Cell Biology 9, 57-60.

Porter, J., Queener, H., Lin, J., Thorn, K., and Awwal, A.A. (2006). Adaptive optics for vision science: Principles, practices, design and applications, Vol 171 (John Wiley \& Sons).

Proll, K.-P., Kohler, C., Baumbach, T., Osten, W., Osten, S., Gruber, H., Langner, A., and Wernicke, G. (2004). Optical characterization of liquid-crystal-on-silicon displays. Proceedings of SPIE 5457.

Rarity, J., and Tapster, P. (1990). Experimental violation of Bell's inequality based on phase and momentum. Physical Review Letters 64, 2495.

Rawls, J.F., and Johnson, S.L. (2000). Zebrafish kit mutation reveals primary and secondary regulation of melanocyte development during fin stripe regeneration. Development 127, 3715-3724.

Ray, K., Ma, J., Oram, M., Lakowicz, J.R., and Black, L.W. (2010). Single Moleculeand Fluorescence Correlation Spectroscopy-FRET Analysis of Phage DNA Packaging: Co-localization of the Packaged Phage T4 DNA Ends within the Capsid. Journal of molecular biology 395, 1102.

Rech, I., Ingargiola, A., Spinelli, R., Labanca, I., Marangoni, S., Ghioni, M., and Cova, S. (2008). Optical crosstalk in single photon avalanche diode arrays: a new complete model. Optics express 16, 8381-8394.

Richardson, J., Walker, R., Grant, L., Stoppa, D., Borghetti, F., Charbon, E., Gersbach, M., and Henderson, R.K. (2009a). A 32× 32 50ps resolution 10 bit time to digital converter array in $130 \mathrm{~nm}$ CMOS for time correlated imaging. Proceedings of IEEE, 77-80.

Richardson, J.A., Grant, L.A., and Henderson, R.K. (2009b). Low dark count single-photon avalanche diode structure compatible with standard nanometer scale CMOS technology. Photonics Technology Letters, IEEE 21, 1020-1022.

Rinnenthal, J.L., Börnchen, C., Radbruch, H., Andresen, V., Mossakowski, A., Siffrin, V., Seelemann, T., Spiecker, H., Moll, I., and Herz, J. (2013). Parallelized TCSPC for dynamic intravital fluorescence lifetime imaging: Quantifying neuronal dysfunction in neuroinflammation. PloS one 8, e60100.

Ripamonti, G., Ghioni, M., and Lacaita, A. (1990). No dead-space optical timedomain reflectometer. Lightwave Technology, Journal of 8, 1278-1283.

Rizzo, M.A., Springer, G.H., Granada, B., and Piston, D.W. (2004). An improved cyan fluorescent protein variant useful for FRET. Nature biotechnology 22, 445449. 
Rocks, O., Peyker, A., Kahms, M., Verveer, P.J., Koerner, C., Lumbierres, M., Kuhlmann, J., Waldmann, H., Wittinghofer, A., and Bastiaens, P.I. (2005). An acylation cycle regulates localization and activity of palmitoylated Ras isoforms. Science 307, 1746-1752.

Roddier, F. (1999). Adaptive optics in astronomy (Cambridge university press).

Rogers, J.A., Paul, K.E., Jackman, R.J., and Whitesides, G.M. (1997). Using an elastomeric phase mask for sub-100 $\mathrm{nm}$ photolithography in the optical near field. Applied Physics Letters 70, 2658-2660.

Roy, R., Hohng, S., and Ha, T. (2008). A practical guide to single-molecule FRET. Nature methods 5, 507-516.

Rozema, J.J., Van Dyck, D.E., and Tassignon, M.-J. (2005). Clinical comparison of 6 aberrometers. Part 1: Technical specifications. Journal of Cataract \& Refractive Surgery 31, 1114-1127.

Rueckel, M., Mack-Bucher, J.A., and Denk, W. (2006). Adaptive wavefront correction in two-photon microscopy using coherence-gated wavefront sensing. Proceedings of the National Academy of Sciences 103, 17137-17142.

Sahoo, H. (2011). Förster resonance energy transfer-A spectroscopic nanoruler: Principle and applications. Journal of Photochemistry and Photobiology C: Photochemistry Reviews 12, 20-30.

Sanders, R., Draaijer, A., Gerritsen, H.C., Houpt, P.M., and Levine, Y.K. (1995). Quantitative $\mathrm{pH}$ imaging in cells using confocal fluorescence lifetime imaging microscopy. Analytical biochemistry 227, 302-308.

Schonbrun, E., Piestun, R., Jordan, P., Cooper, J., Wulff, K.D., Courtial, J., and Padgett, M. (2005). 3D interferometric optical tweezers using a single spatial light modulator. Optics express 13, 3777-3786.

Schwertner, M., Booth, M., and Wilson, T. (2004). Characterizing specimen induced aberrations for high NA adaptive optical microscopy. Optics express 12, 6540-6552.

Schwertner, M., Booth, M., and Wilson, T. (2007). Specimen - induced distortions in light microscopy. Journal of microscopy 228, 97-102.

Schwiegerling, J., and Neal, D.R. (2005). Historical development of the ShackHartmann wavefront sensor. Robert Shannon and Roland Shack: Legends in Applied Optics, edited by JE Harvey and RB Hooker-SPIE, Bellingham, WA, 132-139.

Selvin, P.R. (1995). Fluorescence resonance energy transfer. Methods Enzymol 246, 300-334.

Selvin, P.R. (2000). The renaissance of fluorescence resonance energy transfer. Nature structural biology 7, 730-734. 
Shaner, N.C., Campbell, R.E., Steinbach, P.A., Giepmans, B.N., Palmer, A.E., and Tsien, R.Y. (2004). Improved monomeric red, orange and yellow fluorescent proteins derived from Discosoma sp. red fluorescent protein. Nature biotechnology 22, 1567-1572.

Shaner, N.C., Steinbach, P.A., and Tsien, R.Y. (2005). A guide to choosing fluorescent proteins. Nature methods 2, 905-909.

Shibahara, S., Takeda, K., Yasumoto, K.-i., Udono, T., Watanabe, K.-i., Saito, H., and Takahashi, K. (2001). Microphthalmia-associated transcription factor (MITF): multiplicity in structure, function, and regulation. Journal of Investigative Dermatology, 6, 99-104

Shrauger, V., and Warde, C. (2001). Development of a high-speed high-fill-factor phase-only spatial light modulator. Proceedings of SPIE, 4291.

Siegel, J., Elson, D.S., Webb, S.E., Lee, K., Vlandas, A., Gambaruto, G.L., Leve^queFort, S., Lever, M.J., Tadrous, P.J., and Stamp, G.W. (2003). Studying biological tissue with fluorescence lifetime imaging: microscopy, endoscopy, and complex decay profiles. Applied optics 42, 2995-3004.

Smolek, M.K., and Klyce, S.D. (2003). Zernike polynomial fitting fails to represent all visually significant corneal aberrations. Investigative ophthalmology \& visual science 44, 4676-4681.

So, P.T., Dong, C.Y., Masters, B.R., and Berland, K.M. (2000). Two-photon excitation fluorescence microscopy. Annual review of biomedical engineering 2 , 399-429.

Southwell, W.H. (1980). Wave-front estimation from wave-front slope measurements. JOSA 70, 998-1006.

Spalding, G.C., Courtial, J., and Di Leonardo, R. (2008). Holographic optical tweezers (Academic Press).

Straub, M., and Hell, S. (1998). Fluorescence lifetime three-dimensional microscopy with picosecond precision using a multifocal multiphoton microscope. Applied physics letters 73, 1769-1771.

Stringari, C., Cinquin, A., Cinquin, O., Digman, M.A., Donovan, P.J., and Gratton, E. (2011). Phasor approach to fluorescence lifetime microscopy distinguishes different metabolic states of germ cells in a live tissue. Proceedings of the National Academy of Sciences of the United States of America 108, 1358213587.

Suhling, K., French, P.M., and Phillips, D. (2005). Time-resolved fluorescence microscopy. Photochemical \& Photobiological Sciences 4, 13-22.

Sun, Y., Day, R.N., and Periasamy, A. (2011). Investigating protein-protein interactions in living cells using fluorescence lifetime imaging microscopy. Nature protocols 6, 1324-1340. 
Suyama, M., Hirano, K., Kawai, Y., Nagai, T., Kibune, A., Saito, T., Negi, Y., Asakura, N., Muramatsu, S., and Morita, T. (1997). A hybrid photodetector (HPD) with a III-V photocathode. Proceedings of IEEE, 795-798.

Swann, B.K., Blalock, B.J., Clonts, L.G., Binkley, D.M., Rochelle, J.M., Breeding, E., and Baldwin, K.M. (2004). A 100-ps time-resolution CMOS time-to-digital converter for positron emission tomography imaging applications. Solid-State Circuits, IEEE Journal of 39, 1839-1852.

Talbot, C.B., Patalay, R., Munro, I., Warren, S., Ratto, F., Matteini, P., Pini, R., Breunig, H.G., König, K., and Chu, A.C. (2011). Application of ultrafast gold luminescence to measuring the instrument response function for multispectral multiphoton fluorescence lifetime imaging. Optics express 19, 13848-13861.

Tang, J., Germain, R.N., and Cui, M. (2012). Superpenetration optical microscopy by iterative multiphoton adaptive compensation technique. Proceedings of the National Academy of Sciences of the United States of America 109, 8434-8439.

Tincu, I. (2010). Some properties from Laguerre polynomials. General Mathematics 18, 131-137.

Tosi, A., Mora, A.D., Zappa, F., and Cova, S. (2009). Single-photon avalanche diodes for the near-infrared range: detector and circuit issues. Journal of Modern Optics 56, 299-308.

Trabesinger, W., Hübner, C., Hecht, B., and Wild, U. (2002). Continuous real-time measurement of fluorescence lifetimes. Review of scientific instruments 73 , 3122-3124.

Tyndall, D., Walker, R., Nguyen, K., Galland, R., Gao, J., Wang, I., Kloster, M., Delon, A., and Henderson, R. (2011). Automatic laser alignment for multifocal microscopy using a LCOS SLM and a $32 \times 32$ pixel CMOS SPAD array. Proceedings of SPIE, 8086.

Tyson, R. (2010). Principles of adaptive optics (CRC Press).

Van Munster, E., Goedhart, J., Kremers, G., Manders, E., and Gadella, T.W. (2007). Combination of a spinning disc confocal unit with frequency - domain fluorescence lifetime imaging microscopy. Cytometry Part A 71, 207-214.

Van Rheenen, J., Song, X., Van Roosmalen, W., Cammer, M., Chen, X., DesMarais, V., Yip, S.-C., Backer, J.M., Eddy, R.J., and Condeelis, J.S. (2007). EGF-induced PIP2 hydrolysis releases and activates cofilin locally in carcinoma cells. The Journal of cell biology 179, 1247-1259.

Villa, F., Markovic, B., Bellisai, S., Bronzi, D., Tosi, A., Zappa, F., Tisa, S., Durini, D., Weyers, S., and Paschen, U. (2012). SPAD smart pixel for time-of-flight and timecorrelated single-photon counting measurements. Photonics Journal, IEEE 4, 795-804.

Wallrabe, H., and Periasamy, A. (2005). Imaging protein molecules using FRET and FLIM microscopy. Current opinion in biotechnology 16, 19-27. 
Wang, C., and Ji, N. (2012). Pupil-segmentation-based adaptive optical correction of a high-numerical-aperture gradient refractive index lens for twophoton fluorescence endoscopy. Optics letters 37, 2001-2003.

Wang, X., Dai, H., and Xu, K. (2005). Tunable reflective lens array based on liquid crystal on silicon. Optics express 13, 352-357.

Watson, B.O., Nikolenko, V., and Yuste, R. (2009). Two-photon imaging with diffractive optical elements. Frontiers in neural circuits 3, 6.

Webb, S., Gu, Y., Lévêque-Fort, S., Siegel, J., Cole, M., Dowling, K., Jones, R., French, P., Neil, M., and Juškaitis, R. (2002). A wide-field time-domain fluorescence lifetime imaging microscope with optical sectioning. Review of Scientific Instruments 73, 1898-1907.

Weiner, A.M. (2000). Femtosecond pulse shaping using spatial light modulators. Review of scientific instruments 71, 1929-1960.

Whyte, G., and Courtial, J. (2005). Experimental demonstration of holographic three-dimensional light shaping using a Gerchberg-Saxton algorithm. New Journal of Physics 7, 117.

Williams, R.M., Zipfel, W.R., and Webb, W.W. (2001). Multiphoton microscopy in biological research. Current opinion in chemical biology 5, 603-608.

Wouters, F.S., and Bastiaens, P.I. (1999). Fluorescence lifetime imaging of receptor tyrosine kinase activity in cells. Current biology 9, 1127-S1121.

Wouters, F.S., Verveer, P.J., and Bastiaens, P.I. (2001). Imaging biochemistry inside cells. Trends in cell biology 11, 203-211.

Wright, A.J., Burns, D., Patterson, B.A., Poland, S.P., Valentine, G.J., and Girkin, J.M. (2005). Exploration of the optimisation algorithms used in the implementation of adaptive optics in confocal and multiphoton microscopy. Microscopy research and technique $67,36-44$.

Wyant, J.C., and Creath, K. (1992). Basic wavefront aberration theory for optical metrology. Applied optics and optical engineering 11.

Xi, P., Ren, Q., and Liu, Y. (2011). Scanning and image reconstruction techniques in confocal laser scanning microscopy (INTECH).

Xu, C., and Webb, W.W. (1996). Measurement of two-photon excitation cross sections of molecular fluorophores with data from 690 to $1050 \mathrm{~nm}$. JOSA B 13, 481-491.

Yoon, G., Pantanelli, S., and MacRae, S. (2008). Comparison of Zernike and Fourier wavefront reconstruction algorithms in representing corneal aberration of normal and abnormal eyes. Journal of Refractive Surgery 24, 582.

York, A.G., Chandris, P., Nogare, D.D., Head, J., Wawrzusin, P., Fischer, R.S., Chitnis, A., and Shroff, H. (2013). Instant super-resolution imaging in live cells and embryos via analog image processing. Nature methods 10,1122-1126. 
Yuste, R., and Denk, W. (1995). Dendritic spines as basic functional units of neuronal integration. Nature 375, 682-684.

Zappa, F., Lacaita, A.L., Cova, S.D., and Lovati, P. (1996). Solid - state single photon detectors. Optical Engineering 35, 938-945.

Zappa, F., Tisa, S., Cova, S., Maccagnani, P., Saletti, R., Roncella, R., Baronti, F., Calia, D.B., Silber, A., Bonanno, G., et al. (2007). Photon counting arrays for astrophysics. Journal of Modern Optics 54, 163-189.

Zernike, v.F. (1934). Beugungstheorie des schneidenver-fahrens und seiner verbesserten form, der phasenkontrastmethode. Physica 1, 689-704.

Zipfel, W.R., Williams, R.M., and Webb, W.W. (2003). Nonlinear magic: multiphoton microscopy in the biosciences. Nature biotechnology 21, 13691377.

Zou, W., and Rolland, J.P. (2006). Quantifications of error propagation in slopebased wavefront estimations. JOSA A 23, 2629-2638. 
I would like to express my gratitude to my supervisor, Dr. Simon Ameer-Beg and to Dr. Simon Poland whose expertise, understanding, and patience, added considerably to my graduate experience. I would also like to recognize all members of the Ameer-Beg lab, past and present, whose contribution was also much appreciated.

A special thank you to the Ng Lab, Wells Lab and Geissmann Lab for all their assistance, feedback and fruitful discussions.

I would also like to thank my dearest girlfriend and family for all the support and encouragement

In conclusion, I recognize that this research would not have been possible without the financial assistance of the BBSRC, CRUK and CCIC. 